\title{
Illustration of Sampling-Based Approaches to the Calculation of Expected Dose in Performance Assessments for the Proposed High Level Radioactive Waste Repository at Yucca Mountain, Nevada
}

John C. Helton and Cedric J. Sallaberry

Prepared by

Sandia National Laboratories

Albuquerque, New Mexico 87185 and Livermore, California 94550

Sandia is a multiprogram laboratory operated by Sandia Corpora-

tion, a Lockheed Martin Company, for the United States Department of Energy under Contract DE-AC04-94AL85000.

Approved for public release; further dissemination unlimited. 
Issued by Sandia National Laboratories, operated for the United States Department of Energy by Sandia Corporation.

NOTICE: This report was prepared as an account of work sponsored by an agency of the United States Government. Neither the United States Government, nor any agency thereof, nor any of their employees, nor any of their contractors, subcontractors, or their employees, make any warranty, express or implied, or assume any legal liability or responsibility for the accuracy, completeness, or usefulness of any information, apparatus, product, or process disclosed, or represent that its use would not infringe privately owned rights. Reference herein to any specific commercial product, process, or service by trade name, trademark, manufacturer, or otherwise, does not necessarily constitute or imply its endorsement, recommendation, or favoring by the United States Government, any agency thereof, or any of their contractors or subcontractors. The views and opinions expressed herein do not necessarily state or reflect those of the United States Government, any agency thereof, or any of their contractors.

Printed in the United States of America. This report has been reproduced directly from the best available copy.

Available to DOE and DOE contractors from

U.S. Department of Energy

Office of Scientific and Technical Information

P.O. Box 62

Oak Ridge, TN 37831

Telephone: $\quad$ (865) 576-8401

Facsimile: $\quad$ (865) 576-5728

E-Mail: $\quad$ reports@adonis.osti.gov

Online ordering: http://www.osti.gov/bridge

Available to the public from

U.S. Department of Commerce

National Technical Information Service

5285 Port Royal Rd.

Springfield, VA 22161

Telephone: $\quad$ (800) 553-6847

Facsimile: (703) 605-6900

E-Mail: $\quad$ orders@ntis.fedworld.gov

Online order: $\quad$ http://www.ntis.gov/help/ordermethods.asp?loc=7-4-0\#online

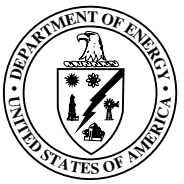




\title{
Illustration of Sampling-Based Approaches to the Calculation of Expected Dose in Performance Assessments for the Proposed High Level Radioactive Waste Repository at Yucca Mountain, Nevada
}

\author{
John C. Helton ${ }^{\mathrm{a}}$ and Cedric J. Sallaberry ${ }^{\mathrm{b}}$ \\ aDepartment of Mathematics and Statistics, Arizona State University, Tempe, AZ 85287-1804 USA \\ bSandia National Laboratories, Albuquerque, NM 87185-0776 USA
}

\begin{abstract}
A deep geologic repository for high level radioactive waste is under development by the U.S. Department of Energy at Yucca Mountain (YM), Nevada. As mandated in the Energy Policy Act of 1992, the U.S. Environmental Protection Agency (EPA) has promulgated public health and safety standards (i.e., 40 CFR Part 197) for the YM repository, and the U.S. Nuclear Regulatory Commission has promulgated licensing standards (i.e., 10 CFR Parts 2, 19, 20, etc.) consistent with 40 CFR Part 197 that the DOE must establish are met in order for the YM repository to be licensed for operation. Important requirements in 40 CFR Part 197 and 10 CFR Parts 2, 19, 20, etc. relate to the determination of expected (i.e., mean) dose to a reasonably maximally exposed individual (RMEI) and the incorporation of uncertainty into this determination. This presentation describes and illustrates how general and typically nonquantitive statements in 40 CFR Part 197 and 10 CFR Parts 2, 19, 20, etc. can be given a formal mathematical structure that facilitates both the calculation of expected dose to the RMEI and the appropriate separation in this calculation of aleatory uncertainty (i.e., randomness in the properties of future occurrences such as igneous and seismic events) and epistemic uncertainty (i.e., lack of knowledge about quantities that are poorly known but assumed to have constant values in the calculation of expected dose to the RMEI).
\end{abstract}

Key Words: Aleatory uncertainty; Epistemic uncertainty; Expected dose; Performance assessment; Radioactive waste disposal; Uncertainty analysis; Yucca Mountain; 10 CFR Parts 2, 19, 20, etc.; 40 CFR Part 197 


\section{Acknowledgments}

Work performed at Sandia National Laboratories (SNL), which is a multiprogram laboratory operated by Sandia Corporation, a Lockheed Martin Company, for the U.S. Department of Energy's National Nuclear Security Administration under Contract No. DE-AC04-04AL85000. Review at SNL provided by T.G. Trucano and R.P. Rechard. Editorial support provided by F. Puffer and J. Ripple of Tech Reps, a division of Ktech Corporation. This presentation is an independent product of the authors and does not necessarily reflect views held by either SNL or the U.S. Department of Energy. 


\section{Contents}

1. Introduction

2. Regulatory Requirements Underlying Conceptual Structure of PAs for YM Repository ..................................13

2.1 EPA Requirements: 40 CFR Part 197 .............................................................................................13

2.2 NRC Requirements: 10 CFR Parts 2, 19, 20, etc. …..........................................................................17

2.3 NRC Review Criteria: Yucca Mountain Review Plan (YMRP)...............................................................24

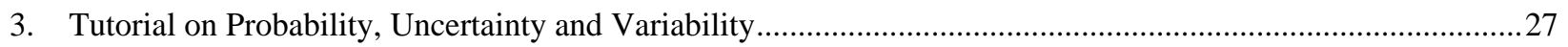

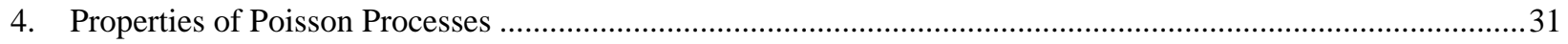

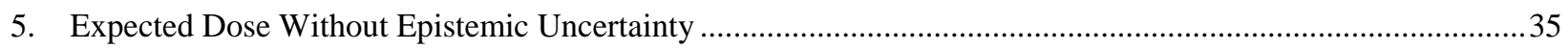

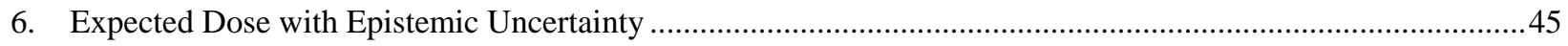

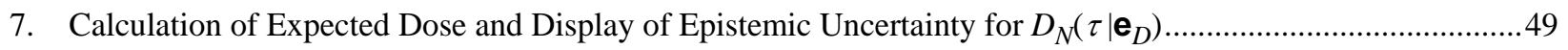

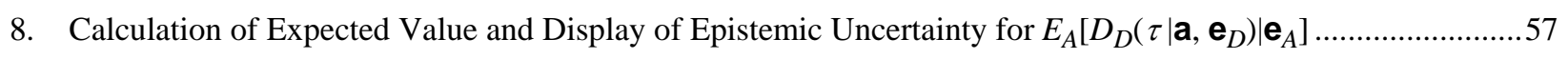

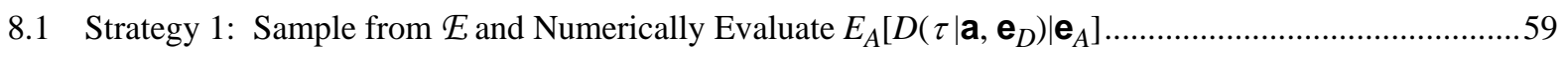

8.2 Strategy 2: Sample from $\mathcal{E} \times \mathscr{P}$ and Numerically Evaluate Integral over Time........................................64

8.3 Strategy 3: Sample from $\mathcal{E} \times \mathcal{P} \times[a, b]$ with Importance Sampling on Time...........................................66

9. Expected Dose Conditional on Single Disruption ...........................................................................................

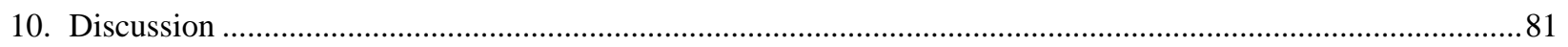

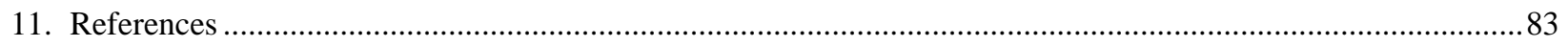




\section{Figures}

Fig. 1. Results for hypothetical dose function $D_{N}\left(\tau \mid \mathbf{e}_{D}\right)$ defined in Eq. (7.9) obtained with a LHS of size $n S$ $=200:(\mathrm{a}, \mathrm{c})$ Individual dose curves $\left[\tau, D_{N}\left(\tau \mid \mathbf{e}_{D i}\right)\right], i=1,2, \ldots, n S=200$, and (b, d) Estimated expected and quantile curves.

Fig. 2. Estimated CDF and CCDF for hypothetical dose function $D_{N}\left(\tau \mid \mathbf{e}_{D}\right)$ defined in Eq. (7.9) at $\tau=5000$ yr obtained with a LHS of size $n S=200$.

Fig. 3. Results for hypothetical dose function $D_{N}\left(\tau \mid \mathbf{e}_{D}\right)$ defined in Eq. (7.9) obtained with $n R=5$ replicated samples of size $n S=100$ with Latin hypercube sampling (a, c, e) and random sampling (b, d, f): (a, b) Estimated expected and quantile curves for individual replicates, (c, d) Estimated CDFs for dose at $5000 \mathrm{yr}$ for individual replicates, and (e, f) 95\% confidence intervals (CIs) for estimated expected values.

Fig. 4. Hypothetical dose curves $D_{D}\left(\tau \mid t_{j}, a_{15}, a_{25}, \mathbf{e}_{D i}\right), j=0,2,4, \ldots, 98$, used in estimation of $E_{A}\left[D_{D}(\tau \mid \mathbf{a}\right.$, $\left.\left.\mathbf{e}_{D 1}\right) \mid \mathbf{e}_{A 1}\right]$ as described in conjunction with Eq. (8.12).

Fig. 5. Representation of epistemic uncertainty associated with hypothetical expected dose $E_{A}\left[D_{D}(\tau \mid \mathbf{a}\right.$, $\left.\left.\mathbf{e}_{D}\right) \mid \mathbf{e}_{A}\right]$ estimated with Strategy 1 and LHS indicated in Eq. (8.10): (a, b) Expected dose curves $\hat{E}_{A}\left[D_{D}\left(\tau \mid \mathbf{a}, \mathbf{e}_{D i}\right) \mid \mathbf{e}_{A i}\right]$ for $i=1,2, \ldots, n S=100$ with linear and log scales, and (c, d) Expected (combined mean) dose $\hat{E}_{E}\left\{E_{A}\left[D_{D}\left(\tau \mid \mathbf{a}, \mathbf{e}_{D}\right) \mid \mathbf{e}_{A}\right]\right\}$ and associated quantiles (i.e., $\left.0.05,0.5,0.95\right)$ for expected dose $E_{A}\left[D_{D}\left(\tau \mid \mathbf{a}, \mathbf{e}_{D}\right) \mid \mathbf{e}_{A}\right]$ defined by $\hat{p}_{E}\left\{E_{A}\left[D_{D}\left(\tau \mid \mathbf{a}, \mathbf{e}_{D}\right) \mid \mathbf{e}_{A}\right] \leq D\right\}$ with linear and log scales.

Fig. 6. Representation of sampling variability in Strategy 1 that results from using $n R=10$ replicated LHSs of size $n S=100$ in the hypothetical estimation of expected (combined mean) dose $E_{E}\left\{E_{A}\left[D_{D}(\tau \mid \mathbf{a}\right.\right.$, $\left.\left.\left.\mathbf{e}_{D}\right) \mid \mathbf{e}_{A}\right]\right\}$ and associated quantiles (i.e., 0.05, 0.5, 0.95) for expected dose $E_{A}\left[D_{D}\left(\tau \mid \mathbf{a}, \mathbf{e}_{D}\right) \mid \mathbf{e}_{A}\right]$ that derive from estimates for $p_{E}\left\{E_{A}\left[D_{D}\left(\tau \mid \mathbf{a}, \mathbf{e}_{D}\right) \mid \mathbf{e}_{A}\right] \leq D\right\}$ : (a) Linear scale, and (b) Log scale.

Fig. 7. Hypothetical estimate obtained with Strategy 1 for expected (combined mean) dose $E_{E}\left\{E_{A}\left[D_{D}(\tau \mid \mathbf{a}\right.\right.$, $\left.\left.\left.\mathbf{e}_{D}\right) \mid \mathbf{e}_{A}\right]\right\}$ that derives from $n R=10$ replicated LHSs of size $n S=100$ and associated 95\% confidence intervals (CIs) obtained with the $t$-distribution as indicated in conjunction with Eq. (7.19).

Fig. 8. Hypothetical dose curves $D_{D}\left(\tau \mid t_{j}, a_{11}, a_{21}, \mathbf{e}_{D 1}\right), j=0,2,4, \ldots, 98$, for sample element $\mathbf{e}_{1}$ of sample in Eq. (8.26) used in estimation of conditional expected dose $E_{t}\left[D_{D}\left(\tau \mid \mathbf{p}_{1}, \mathbf{e}_{D 1}, \lambda_{D 1}\right)\right]$ with Strategy 2 as described in conjunction with Eq. (8.23).

Fig. 9. Hypothetical estimates obtained with Strategy 2 for conditional expected dose $E_{t}\left[D_{D}\left(\tau \mid \mathbf{p}_{i}, \mathbf{e}_{D i}, \lambda_{D i}\right)\right]$, $i=1,2, \ldots, n S=100$, and expected (combined mean) dose $E_{E}\left\{E_{A}\left[D_{D}\left(\tau \mid \mathbf{a}, \mathbf{e}_{D}\right) \mid \mathbf{e}_{A}\right]\right\}$ obtained as described in Eqs. (8.23) and (8.24) for the sample in Eq. (8.26): (a) Linear scale, and (b) Log scale........66

Fig. 10. Representation of sampling variability that results from using $n R=10$ replicated LHSs of size $n S=$ 100 and $n S=500$ in the hypothetical estimation of expected (combined mean) dose $E_{E}\left\{E_{A}\left[D_{D}(\tau \mid \mathbf{a}\right.\right.$, $\left.\left.\left.\mathbf{e}_{D}\right) \mid \mathbf{e}_{A}\right]\right\}$ with Strategy 2: (a, b) Replicated estimates $\hat{E}_{E r}\left\{E_{A}\left[D_{D}\left(\tau \mid \mathbf{a}, \mathbf{e}_{D}\right) \mid \mathbf{e}_{A}\right]\right\}, r=1,2, \ldots, 10$, and (c, d) Estimates for $E_{E}\left\{E_{A}\left[D_{D}\left(\tau \mid \mathbf{a}, \mathbf{e}_{D}\right) \mid \mathbf{e}_{A}\right]\right\}$ and associated $95 \%$ confidence intervals (CIs) that derive from the replicated estimates $E_{E r}\left\{E_{A}\left[D_{D}\left(\tau \mid \mathbf{a}, \mathbf{e}_{D}\right) \mid \mathbf{e}_{A}\right]\right\}$.

Fig. 11. Linear scale plots for unweighted and weighted hypothetical doses obtained with samples of size $n S=$ 100 of the form indicated in Eq. (8.35) and used in the approximation of expected (combined mean) dose $E_{E}\left\{E_{A}\left[D_{D}\left(\tau \mid \mathbf{a}, \mathbf{e}_{D}\right) \mid \mathbf{e}_{A}\right]\right\}$ defined in Eq. (8.29) with Strategy 3: (a) $D_{D}\left(\tau \mid t_{i}, \mathbf{p}_{i}, \mathbf{e}_{D i}\right), i=1,2$, ..., 100, obtained with uniform sampling on time, (b) $\left[(10,000-10) \lambda_{D i}\right] D_{D}\left(\tau \mid t_{i}, \mathbf{p}_{i}, \mathbf{e}_{D i}\right), i=1,2$, $\ldots, 100$, obtained with uniform sampling on time, (c) $D_{D}\left(\tau \mid t_{i}, \mathbf{p}_{i}, \mathbf{e}_{D i}\right), i=1,2, \ldots, 100$, obtained with loguniform sampling on time, and (d) $\left[\lambda_{D i} t_{i} \ln (10,000 / 10)\right] D_{D}\left(\tau \mid t_{i}, \mathbf{p}_{i}, \mathbf{e}_{D i}\right), i=1,2, \ldots, 100$, obtained with loguniform sampling on time.

Fig. 12. Log scale plots for unweighted and weighted hypothetical doses obtained with same samples of size $n S=100$ of the form indicated in Eq. (8.35) and used in the approximation of expected (combined mean) dose $E_{E}\left\{E_{A}\left[D_{D}\left(\tau \mid \mathbf{a}, \mathbf{e}_{D}\right) \mid \mathbf{e}_{A}\right]\right\}$ defined in Eq. (8.29) with Strategy 3: (a) $D_{D}\left(\tau \mid t_{i}, \mathbf{p}_{i}, \mathbf{e}_{D i}\right), i=$ $1,2, \ldots, 100$, obtained with uniform sampling on time, (b) [(10,000 - 10) $\left.\lambda_{D i}\right] D_{D}\left(\tau \mid t_{i}, \mathbf{p}_{i}, \mathbf{e}_{D i}\right), i=1$, $2, \ldots, 100$, obtained with uniform sampling on time, (c) $D_{D}\left(\tau \mid t_{i}, \mathbf{p}_{i}, \mathbf{e}_{D i}\right), i=1,2, \ldots, 100$, obtained with loguniform sampling on time, and (d) $\left[\lambda_{D i} t_{i} \ln (10,000 / 10)\right] D_{D}\left(\tau \mid t_{i}, \mathbf{p}_{i}, \mathbf{e}_{D i}\right), i=1,2, \ldots, 100$, obtained with loguniform sampling on time. 
Fig. 13. Representation of sampling variability that results from using $n R=10$ replicated LHSs of size $n S=$ $100,500,1000$ and 5000 in the hypothetical estimation of expected (combined mean) dose $E_{E}\left\{E_{A}\left[D_{D}\left(\tau \mid \mathbf{a}, \mathbf{e}_{D}\right) \mid \mathbf{e}_{A}\right]\right\}$ with Strategy 3 and either uniform (a,c,e,g) or loguniform (b,d,f,h) sampling on time.

Fig. 14. Ninety-five percent (i.e., 95\%) confidence intervals (CIs) for hypothetical estimates of expected (combined mean) dose $E_{E}\left\{E_{A}\left[D_{D}\left(\tau \mid \mathbf{a}, \mathbf{e}_{D}\right) \mid \mathbf{e}_{A}\right]\right\}$ with Strategy 3 that result from using $n R=10$ replicated LHSs of sizes $n S=100,500,1000$ and 5000 and either uniform (a,c,e,g) or loguniform (b,d,f,h) sampling on time.

Fig. 15. Combined epistemic and aleatory uncertainty in hypothetical dose conditional on exactly one disruptive occurrence taking place in the time interval $[a, b]=[10,20,000 \mathrm{yr}]$ summarized as expected (combined conditional mean) dose $\hat{E}_{E \cup A}\left[D_{D}(\tau) \mid \mathbf{a} \in \mathcal{A}_{1}(a, b)\right]$ and associated quantiles (i.e., $0.05,0.5,0.95)$ for $D_{D}\left(\tau \mid t, \mathbf{p}, \mathbf{e}_{D}\right)$ conditional on $\mathbf{a}=[t, \mathbf{p}] \in \mathcal{A}_{1}(a, b)$ defined by $\hat{p}_{E \cup A}\left[D_{D}(\tau)<D \mid \mathbf{a}\right.$ $\left.\in \mathcal{A}_{1}(a, b)\right]$ obtained with Strategy 3 for the evaluation of expected (combined mean) dose $E_{E}\left\{E_{A}\left[D_{D}\left(\tau \mid \mathbf{a}, \mathbf{e}_{D}\right) \mid \mathbf{e}_{A}\right]\right\}$ and LHSs of size $n S=1000$ and 5000: (a,c) Uniform sampling on time and use of approximations in Eqs. (9.5) and (9.6), and (b,d) Loguniform sampling on time and use of approximations in Eqs. (9.9) and (9.10).

Fig. 16. Combined epistemic and aleatory uncertainty in hypothetical dose conditional on exactly one disruptive occurrence taking place in the time interval $[a, b]=[10,20,000 \mathrm{yr}]$ summarized as expected (combined conditional mean) dose $\hat{E}_{E \cup A}\left[D_{D}(\tau) \mid \mathbf{a} \in \mathcal{A}_{1}(a, b)\right]$ and associated quantiles (i.e., $0.05,0.5,0.95)$ for $D_{D}\left(\tau \mid t, \mathbf{p}, \mathbf{e}_{D}\right)$ conditional on $\mathbf{a}=[t, \mathbf{p}] \in \mathcal{A}_{1}(a, b)$ defined by $\hat{p}_{E \cup A}\left[D_{D}(\tau)<D \mid \mathbf{a}\right.$ $\left.\in \mathcal{A}_{1}(a, b)\right]$ obtained with results from (i) Strategy 1 as indicated in Eqs. (9.15) and (9.16) with LHS of size 100, (ii) Strategy 2 as indicated in Eqs. (9.18) and (9.19) with LHS of size 100, and (iii) Strategy 3 as indicated in Eqs. (9.9) and (9.10) with LHSs of size 5000 and outcomes shown for both uniform and loguniform sampling on time (i.e., there are two sets of curves for Strategy 3): (a) Linear scale, and (b) Log scale.

\section{Tables}

Table 1. Illustration of Steps Leading to Integral Representation for $E_{A}[D(\tau \mid \mathbf{a})]$ in Eq. (5.24)

Table 2. Distributions Characterizing Epistemic Uncertainty in the Variables $e_{D 1}, e_{D 2}, \ldots, e_{D 5}$ Used in the Definition of $D_{N}\left(\tau \mid \mathbf{e}_{D}\right)$ in Eq. (7.9)

Table 3. Distributions Characterizing Epistemic Uncertainty in the Variables $e_{D 6}, e_{D 7}$ and $e_{D 8}$ Used in the Definition of $D_{N}\left(\tau \mid t, \mathbf{p}, \mathbf{e}_{D}\right)$ in Eq. (8.3) (see Table 2 for distributions for $\left.e_{D 1}, e_{D 2}, \ldots, e_{D 5}\right)$ and in the Variables $e_{A 1}, e_{A 2}$ and $e_{A 3}$ Used in the Characterization of Aleatory Uncertainty in $a_{1}, a_{2}$ and $t$ 58 
This page intentionally left blank. 


\section{Acronyms}

CCDF complementary cumulative distribution function

$\mathrm{CDF}$

DOE

EPA

LHS

NRC

PA

RMEI

SNL cumulative distribution function

U.S. Department of Energy

U.S. Environmental Protection Agency

Latin hypercube sample

U.S. Nuclear Regulatory Commission

performance assessment

reasonably maximally exposed individual

TSPA-LA

WIPP

YM

Total System Performance Assessment-License Application

Waste Isolation Pilot Plant

YMRP

Yucca Mountain

Yucca Mountain Review Plan 
This page intentionally left blank. 


\section{Introduction}

The appropriate disposal of radioactive waste from military and commercial activities is a challenge of national and international importance. ${ }^{1-13}$ As part of the solution to this challenge, a proposed deep geologic repository for high-level radioactive waste is under development by the U.S. Department of Energy (DOE) at Yucca Mountain (YM), Nevada. ${ }^{14-18}$ The development of the YM repository is the single most important radioactive waste disposal project currently being undertaken in the United States.

As mandated in the Energy Policy Act of 1992, ${ }^{19}$ the U.S. Environmental Protection Agency (EPA) is required to promulgate public health and safety standards for radioactive material stored or disposed of in the YM repository; the U.S. Nuclear Regulatory Commission (NRC) is required to incorporate the EPA standards into licensing standards for the YM repository; and the DOE is required to show compliance with the NRC standards. The regulatory requirements for the YM repository that resulted from these mandates have three primary sources: (i) Public Health and Environmental Protection Standards for Yucca Mountain, NV; Final Rule (40 CFR Part 197), ${ }^{20}$ which has been promulgated by the EPA, (ii) Disposal of High-Level Radioactive Wastes in a Proposed Geologic Repository at Yucca Mountain, Nevada; Final Rule (10 CFR Parts 2, 19, 20, etc.), ${ }^{21}$ which has been promulgated by the NRC, and (iii) Yucca Mountain Review Plan; Final Report (YMRP), ${ }^{22}$ which has been published by the NRC to guide assessing compliance with 10 CFR Parts 2, 19, 20, etc. In turn, the DOE is required to carry out a performance assessment (PA) for the YM repository that satisfies the requirements specified in the preceding three documents.

A major requirement imposed by the preceding three documents on PAs carried out by the DOE for the YM repository is to determine expected dose to a reasonably maximally exposed individual (RMEI). However, the indicated documents are not completely clear from a mathematical, and hence computational, perspective as to exactly what this expected dose should be. In particular, the determination of an expected value requires the introduction of an appropriate probabilistic structure (i.e., a probability space) and then the evaluation of an integral involving this probabilistic structure. However, rather than making precise statements about the probabilistic structure that must underlie the determination of the expected dose to the RMEI and also about the nature of the integration processes that must be carried out to obtain this expected dose, the indicated documents rely on very general statements to express what is desired. This generality has the benefit of allowing a significant level of freedom in planning and implementing a PA for the YM repository and thus in determining expected dose to the RMEI. Unfortunately, this generality has also resulted in a considerable amount of confusion with respect to exactly what the desired expected dose is and how it should be calculated.

The purpose of this presentation is to clearly describe how expected dose can be defined and calculated. Particular attention is paid to the roles that the probabilistic characterizations of aleatory and epistemic uncertainty ${ }^{23-32}$ play in the definition, calculation and display of expected dose. Expected dose is formally defined by appropriate integrals and then several alternative procedures for the evaluation of these integrals are presented and discussed. Much of the confusion involving the definition and determination of expected dose has resulted from the use of different procedures to evaluate these integrals. Because these procedures are based on different numerical algorithms, superficially similar intermediate results obtained in the determination of expected dose to the RMEI with these procedures can correspond to very different quantities. A failure to recognize these differences has led, at times, to misinterpretations of results and, at other times, to outright errors. For example, the use of importance sampling in the evaluation of the integrals defining expected dose has been a source of considerable confusion and misinterpretation because of misconceptions about the properties of individual observations generated in this sampling process. A major emphasis of this presentation is the appropriate interpretation of intermediate "dose curves" obtained in the numerical calculation of expected dose to the RMEI. To allow concentration on concept rather than computational detail and to simplify the presentation, simple illustrative examples are used rather than actual results from a full PA for the YM repository.

The presentation is organized as follows. First, the regulatory requirements that result in the need to determine expected dose to the RMEI in PAs for the YM repository are summarized (Sect. 2). Then, a brief discussion is given on the connections between the uncertainty representations requested by the EPA and the NRC and basic concepts in the representation of uncertainty with probability (Sect. 3). Next, because of their importance in the deter- 
mination of expected dose to the RMEI, properties of Poisson processes are briefly summarized (Sect. 4). Then, the following core topics are considered: (i) determination of expected dose without consideration of epistemic uncertainty (Sect. 5), (ii) determination of expected dose with consideration of epistemic uncertainty (Sect. 6), (iii) calculation of expected dose and display of epistemic uncertainty for nominal (i.e., undisturbed) conditions (Sect.7), and (iv) calculation of expected dose and display of epistemic uncertainty for disturbed conditions (Sect. 8). The important distinction between the results in Sects. 7 and 8 is that the nominal conditions considered in Sect. 7 do not involve a treatment of aleatory uncertainty while the disturbed conditions considered in Sect. 8 involve a treatment of both aleatory and epistemic uncertainty. In addition, three different computational strategies are discussed in Sect. 8 for the evaluation of the integrals that define expected dose in the presence of both aleatory and epistemic uncertainty. A description is also given on the use of results obtained with the individual computational strategies in the determination of expected dose conditional on the assumption that exactly one disruption has occurred in a specified time interval (Sect. 9). Finally, the presentation ends with a concluding discussion (Sect. 10).

Additional background on the proposed YM repository is available in a series of technical reports (e.g., 14-18) and also in a rapidly growing body of journal literature (e.g., 33-50). 


\section{Regulatory Requirements Underlying Conceptual Structure of PAs for YM Repository}

\subsection{EPA Requirements: 40 CFR Part 197}

The EPA mandates standards for the YM repository addressing individual protection (i.e., 40 CFR 197.20), human intrusion (i.e., 40 CFR 197.25), and groundwater protection (i.e., 40 CFR 197.30). The individual protection standard is the core requirement in 40 CFR 197 in that it ultimately gives rise to the overall conceptual and computational structure of PAs for the YM repository. Specifically, the following individual-protection standard is mandated (Ref. [20], p. 32134):

\section{§ 197.20 What standard must DOE meet?}

The DOE must demonstrate, using performance assessment, that there is a reasonable expectation that, for 10,000 years following disposal, the reasonably maximally exposed individual receives no more than an annual committed effective dose equivalent of 150 microsieverts (15 millirems) from releases from the undisturbed Yucca Mountain disposal system. The DOE's analysis must include all potential pathways of radionuclide transport and exposure.

A reading of the preceding requirement immediately gives rise to four questions: (i) What is a PA?, (ii) What is reasonable expectation?, (iii) What is the reasonably maximally exposed individual (RMEI)?, and (iv) What is the undisturbed YM disposal system?.

In answer to the first question, the EPA defines a PA in 40 CFR Part 197 in the statement (Ref. [20], p. 32133):

Performance assessment means an analysis that:

(1) Identifies the features, events, processes, (except human intrusion), and sequences of events and processes (except human intrusion) that might affect the Yucca Mountain disposal system and their probabilities of occurring during 10,000 years after disposal;

(2) Examines the effects of those features, events, processes, and sequences of events and processes upon the performance of the Yucca Mountain disposal system; and

(3) Estimates the annual committed effective dose equivalent incurred by the reasonably maximally exposed individual, including the associated uncertainties, as a result of releases caused by all significant features, events, processes, and sequences of events and processes, weighted by their probability of occurrence.

In answer to the second question, the EPA defines reasonable expectation in 40 CFR Part 197 in the statement (Ref. [20], p. 32133):

\section{$\S 197.14$ What is a reasonable expectation?}

Reasonable expectation means that NRC is satisfied that compliance will be achieved based upon the full record before it. Characteristics of reasonable expectation include that it:

(a) Requires less than absolute proof because absolute proof is impossible to attain for disposal due to the uncertainty of projecting long-term performance;

(b) Accounts for the inherently greater uncertainties in making long-term projections of the performance of the Yucca Mountain disposal system; 
(c) Does not exclude important parameters from assessments and analyses simply because they are difficult to precisely quantify to a high degree of confidence; and

(d) Focuses performance assessments and analyses upon the full range of defensible and reasonable parameter distributions rather than only upon extreme physical situations and parameter values.

In answer to the third question, the EPA defines the RMEI in 40 CFR Part 197 in the statement (Ref. [20], p. 32134):

\section{$\S 197.21$ Who is the reasonably maximally exposed individual?}

The reasonably maximally exposed individual is a hypothetical person who meets the following criteria:

(a) Lives in the accessible environment above the highest concentration of radionuclides in the plume of contamination;

(b) Has a diet and living style representative of the people who now reside in the Town of Amargosa Valley, Nevada. The DOE must use projections based upon surveys of the people residing in the Town of Amargosa Valley, Nevada, to determine their current diets and living styles and use the mean values of these factors in the assessments conducted for $\S \S 197.20$ and 197.25; and

(c) Drinks 2 liters of water per day from wells drilled into the ground water at the location specified in paragraph (a) of this section.

Finally, in answer to the fourth question, the EPA defines the undisturbed YM disposal system in the statement (Ref. [20], p. 32133):

Undisturbed Yucca Mountain disposal system means that the Yucca Mountain disposal system is not affected by human intrusion.

With respect to the preceding statements, the definitions of PA and reasonable expectation are particularly important with respect to the conceptual structure of PAs for the YM facility because they relate to requirements that affect the overall organization of the analysis. In contrast, the definition of the RMEI is less important to the conceptual structure of the analysis because it simply relates to a particular result that must be calculated. Further, it is important to recognize that the requirement to consider "the undisturbed Yucca Mountain disposal system" only pertains to human disturbances; natural disturbances such as seismic and igneous events are not excluded. However, the following provision places a bound on the likelihood of such occurrences for inclusion in a PA for the YM repository (Ref. [20], p. 32135):

The DOE's performance assessments shall not include very unlikely features, events, or processes, i.e., those that are estimated to have less than one chance in 10,000 of occurring within 10,000 years of disposal.

(EPA6)

A PA is often described as an analysis intended to answer three questions about a system and one additional question about the analysis itself. ${ }^{32,}$ 51-53 The first three questions are:

(i) What could happen?

(ii) How likely is it to happen?

and 
(iii) What are the consequences if it does happen?

The fourth question is:

(iv) What is the uncertainty (or equivalently, how much confidence do you have) in the answers to the first three questions?

The posing of the first three questions in the definition of a PA in Quote (EPA2) is clearly evident. Specifically, "Identifies features, events and processes" corresponds to answering Question (Q1) and "Identifies ... their probabilities of occurring" corresponds to answering Question (Q2). Similarly, "Examines the effects of those features, events and processes" and "Estimates the annual committed effective dose equivalent" correspond to answering Question (Q3). The presence of Question (Q4) is not as immediately obvious but is imbedded in the definition of PA in Quote (EPA2) in the statement "including the associated uncertainties" and in the definition of reasonable expectation in Quote (EPA3) in the statements "Accounts for the inherently greater uncertainties in making longterm projections" and "Focuses performance assessments upon the full range of defensible and reasonable parameter values". Questions (Q1) - (Q4) are introduced here because they will later be used as an intuitive lead into the conceptual structure of PAs for the YM repository.

The EPA provides the following guidance on the implementation of the post-closure requirements in 40 CFR 197.13 (Ref. [20], p. 32133):

\section{$\S$ 197.13 How is subpart B implemented?}

The NRC implements this subpart B. The DOE must demonstrate to NRC that there is a reasonable expectation of compliance with this subpart before NRC may issue a license. In the case of the specific numerical requirements in $\S 197.20$ of this subpart, and if performance assessment is used to demonstrate compliance with the specific numerical requirements in $\S \S$ 197.25 and 197.30 of this subpart, NRC will determine compliance based upon the mean of the distribution of projected doses of DOE's performance assessments which project the performance of the Yucca Mountain disposal system for 10,000 years after disposal.

Of particular importance is the statement that "In the case of the specific numerical requirements in $\S 197.20 \ldots$, NRC will determine compliance based upon the mean of projected doses." This is a very important requirement with respect to the conceptual and computational structure of a PA for the YM repository, and a requirement that is not easy to interpret. In particular, a mean is a quantity that is defined by an integral, and it is not immediately obvious exactly what set of values this integral should be taken over.

The requirement to consider means can be traced back to guidance given in the report Technical Bases for Yucca Mountain Standards prepared under the auspices of the National Research Council (Ref. [54], p. 123). As directed in the Energy Policy Act of 1992 (Ref. [19], Sect. 801), this report was prepared to assist the EPA in developing standards for the YM repository.

Identifying what is intended by the "mean" indicated in Quote (EPA7) is complicated by the required consideration of two types of uncertainty in the EPA mandated PA for the YM repository. First, there is the uncertainty that arises from the different types of disruptions that could occur at the YM repository in the future. This uncertainty appears in the EPA's definition of PA in Quote (EPA2) in the statements "Identifies features, events and processes ... and their probabilities of occurring during 10,000 years after disposal" and "Estimates the annual effective dose equivalent ... as a result of releases caused by all significant features, events and processes, and sequences of events and processes, weighted by their probability of occurrence.” The latter statement appears to imply a mean, i.e., an integral, over what could happen in the future. This is the uncertainty associated with Questions (Q1) and (Q2). Second, there is the uncertainty that arises from a lack of knowledge about properties of the YM site. This uncertainty appears in the EPA's definition of PA in Quote (EPA2) in the statement "including the associated uncertainties" and in the definition of reasonable expectation in Quote (EPA3) in the statements "due to the uncertainty of projecting long-term performance" and "the full range of defensible and reasonable parameter distri- 
butions." Given that these uncertainties are to be characterized probabilistically as implied by the last quote, then there would also be a mean over these uncertainties. These are the uncertainties associated with Question (Q4).

Possibilities include a mean over what could happen in the future, a mean over uncertainty with respect to parameter values and other modeling assumptions, and a mean over both types of uncertainty. When read in full, the following final part of the definition of PA in Quote (EPA2) suggests, but does not explicitly state, the last of the three indicated possibilities: "Estimates the annual committed effective dose equivalent incurred by the reasonably maximally exposed individual, including the associated uncertainties, as a result of releases caused by all significant features, events, processes and sequences of events and processes, weighted by their probability of occurrence," with the italics added for emphasis. As a further consideration, doses to the RMEI vary as a function of time, and this variation must be incorporated in some manner in the determination of mean doses.

The EPA does not provide a definitive statement in 40 CFR Part 197 of what is intended by a mean dose. However, a number of additional statements are included in the supplementary information accompanying 40 CFR Part 197 in the Federal Register that indicate the EPA's emphasis on consideration of a mean dose in assessing compliance:

In line with our use of the term "reasonable expectation," the fundamental compliance measure consistent with a literal mathematical interpretation of this term would be the mean value of the distribution of calculated doses. (Ref. [20], p. 32125)

By specifying the mean as the performance measure and probability limits for the processes and events to be considered (§ 197.36), and in concert with the intent of our "reasonable expectation", approach in general, we have implied that probabilistic approaches for the disposal system performance assessments are expected. (Ref. [20], p. 32125)

The mean or median are reasonably conservative measures because they are influenced by high exposure estimates found when analyzing the full range of site conditions and relevant processes, without being geared to exclusively reflect high-end results, as would be the case if we selected as the measure a high-end percentile of the calculated dose distribution (such as the 95th or 99th percentile). Our final rule for Yucca Mountain specifies only that the mean be used, as we believe that it is appropriately conservative in this situation. (Ref. [20], p. 32126)

Thus, although some ambiguity exists with respect to exactly how a mean dose is to be defined and calculated, there is no ambiguity in the EPA's intent that compliance with its dose standard for the RMEI is to be determined with a mean dose. As an aside, it is perhaps worth noting that the statement with respect to the median in Quote (EPA10) is not correct for distributions that are skewed toward large values; for such distributions, the median unlike the mean is not particularly influenced by the high values associated with the upper tail of the distribution.

The discussion of the requirements promulgated by the EPA in 40 CFR Part 197 ends with several additional quotes that provide insights into the nature of the desired properties of a PA used to assess compliance with the EPA’s YM standard:

It is NRC's responsibility to determine how DOE must demonstrate compliance with our standards; however, we envision the use of a probabilistic assessment for the compliance demonstration. (Ref. [20], p. 32086))

DOE and NRC may not assume that future geologic, hydrologic, and climatic conditions will be the same as they are at present. We require that these conditions be varied within reasonably ascertainable bounds over the required compliance period. (Ref. [20], p. 32096)

If choices are made that make the simulations very unrealistic, the confidence that can be placed on modeling results is very limited. Inappropriate simplifications can mask the effects of processes that will in reality determine disposal system performance, if the uncertainties involved 
with these simplifications are not recognized. Overly conservative assumptions made in developing performance scenarios can bias the analyses in the direction of unrealistically extreme situations, which in reality may be highly improbable, and can deflect attention from questions critical to developing an adequate understanding of the expected features, events, and processes. (Ref. [20], p. 32102)

The reasonable expectation approach is aimed simply at focusing attention on understanding the uncertainties in projecting disposal system performance so that regulatory decision making will be done with a full understanding of the uncertainties involved. (Ref. [20], p. 32102)

Quote (EPA11) clearly indicates that the EPA expects a probabilistic analysis. Quote (EPA12) indicates the need to consider events that can happen in the future (e.g., volcanic eruptions, earthquakes, climatic changes). Quote (EPA13) is important because it clearly indicates that the EPA does not desire a conservative analysis. Quote (EPA14) indicates the EPA's desire for an appropriate representation of the uncertainties associated with a PA for the YM site. However, the EPA's desire "that regulatory decision making will be done with a full understanding of the uncertainties involved" as stated in Quote (EPA14) is not fully consistent with the intent to regulate on mean results as the calculation of means suppresses the implications of uncertainty.

\subsection{NRC Requirements: 10 CFR Parts 2, 19, 20, etc.}

As mandated in the Energy Policy Act of 1992, the NRC has promulgated licensing standards for the disposal of radioactive waste at the YM repository that are consistent with the EPA public health and safety standards in 40 CFR Part 197. Selected requirements and explanatory material from 10 CFR Parts 2, 19, 20, etc. that influence the conceptual and computational structure of PAs for the YM repository for post-closure conditions follow. In particular, the primary focus is on Subpart L of 10 CFR Part 63, which relates to post-closure requirements.

The core requirement in 10 CFR Part 63 that ultimately gives rise to the conceptual and computational structure of PAs for the YM repository is an individual protection standard. Specifically, the following standard is mandated (Ref. [21], p. 55814):

\section{§ 63.311 Individual protection standard after permanent closure.}

DOE must demonstrate, using performance assessment, that there is a reasonable expectation that, for 10,000 years following disposal, the reasonably maximally exposed individual receives no more than an annual dose of $0.15 \mathrm{mSv}(15 \mathrm{mrem})$ from releases from the undisturbed Yucca Mountain disposal system. DOE's analysis must include all potential pathways of radionuclide transport and exposure.

The preceding mandate is essentially identical to the corresponding mandate from the EPA in Quote (EPA1) provided the terminology in the two mandates has the same meaning. In particular, the phrases performance assessment, reasonable expectation, reasonably maximally exposed individual (RMEI), and undisturbed YM disposal system appear in both mandates.

Specifically, the NRC defines PA in 10 CFR Part 63.2 in the statement (Ref. [21], p. 55794):

Performance assessment means an analysis that:

(1) Identifies the features, events, processes (except human intrusion), and sequences of events and processes (except human intrusion) that might affect the Yucca Mountain disposal system and their probabilities of occurring during 10,000 years after disposal;

(2) Examines the effects of those features, events, processes, and sequences of events and processes upon the performance of the Yucca Mountain disposal system; and 
(3) Estimates the dose incurred by the reasonably maximally exposed individual, including the associated uncertainties, as a result of releases caused by all significant features, events, processes, and sequences of events and processes, weighted by their probability of occurrence.

(NRC2)

The preceding definition of PA is identical to the definition given by the EPA in Quote (EPA2) except for the use of "dose" rather than "annual committed effective dose"; presumably, the NRC intends the same dose measure as specified by the EPA.

Further, the NRC defines reasonable expectation in the statement (Ref. [21], p. 55813):

\section{§ 63.304 Reasonable expectation.}

Reasonable expectation means that the Commission is satisfied that compliance will be achieved based upon the full record before it. Characteristics of reasonable expectation include that it:

(1) Requires less than absolute proof because absolute proof is impossible to attain for disposal due to the uncertainty of projecting long-term performance;

(2) Accounts for the inherently greater uncertainties in making long-term projections of the performance of the Yucca Mountain disposal system;

(3) Does not exclude important parameters from assessments and analyses simply because they are difficult to precisely quantify to a high degree of confidence; and

(4) Focuses performance assessments and analyses on the full range of defensible and reasonable parameter distributions rather than only upon extreme physical situations and parameter values.

The preceding is identical to the EPA's definition of reasonable expectation in Quote (EPA3) except for the replacement of "NRC" by "the Commission."

The RMEI defined by the NRC in the statement (Ref. [21], p. 55814):

\section{§ 63.312 Required characteristics of the reasonably maximally exposed individual.}

The reasonably maximally exposed individual is a hypothetical person who meets the following criteria:

(a) Lives in the accessible environment above the highest concentration of radionuclides in the plume of contamination;

(b) Has a diet and living style representative of the people who now reside in the Town of Amargosa Valley, Nevada. DOE must use projections based upon surveys of the people residing in the Town of Amargosa Valley, Nevada, to determine their current diets and living styles and use the mean values of these factors in the assessments conducted for $\S \S$ 63.311 and 63.321;

(c) Uses well water with average concentrations of radionuclides based on an annual water demand of 3000 acre-feet;

(d) Drinks 2 liters of water per day from wells drilled into the ground water at the location specified in paragraph (a) of this section; and 
(e) Is an adult with metabolic and physiological considerations consistent with present knowledge of adults.

(NRC4)

Except for some additional guidance on the determination of exposure, the definition of the RMEI given by the NRC is the same as the definition given by the EPA in Quote (EPA4).

Finally, the NRC defines the undisturbed YM disposal system in the statement (Ref. [21], p. 55813):

Undisturbed Yucca Mountain disposal system means that the Yucca Mountain disposal system is not affected by human intrusion.

(NRC5)

The preceding definition for the undisturbed YM disposal system is the same as the EPA definition in Quote (EPA5).

Further, like the EPA, the NRC excludes very unlikely features, events and processes from consideration in PAs for the YM repository (Ref. [21], p. 55815; see also Ref. [55], p. 62634):

DOE's performance assessments should not include very unlikely features, events, or processes, i.e., those estimated to have less than one chance in 10,000 of occurring within 10,000 years of disposal.

As a result of the effectively identical content of the requirements/definitions given by the NRC in Quotes (NRC1) - (NRC5) and the EPA in Quotes (EPA1) - (EPA5), both organizations are specifying the same analysis. Hence, the four core questions underlying a PA indicated in Questions (Q1) - (Q4) are present in the requirements of both the NRC and the EPA.

Because the NRC has regulatory authority for the YM repository, it is important to examine any additional requirements and/or specifications that it has made with respect to PA in support of a license application for this facility. In particular, the following guidance on the implementation of post-closure requirements provides a high-level summary of what the NRC expects in a PA for the YM repository (Ref. [21], p. 55813):

\section{§ 63.303 Implementation of Subpart L.}

DOE must demonstrate that there is a reasonable expectation of compliance with this subpart before a license may be issued. In the case of the specific numerical requirements in $\S 63.311$ of this subpart, and if performance assessment is used to demonstrate compliance with the specific numerical requirements in $\S \S 63.321$ and 63.331 of this subpart, compliance is based upon the mean of the distribution of projected doses of DOE's performance assessments which project the performance of the Yucca Mountain disposal system for 10,000 years after disposal.

Except for minor changes in wording, the preceding implementation guidance is identical with the guidance given by the EPA in Quote (EPA7). Of particular importance is the statement that "compliance is based upon the mean of the distribution of projected doses.” As discussed in conjunction with Quote (EPA7), this requirement has a major effect on the conceptual and computational structure of the TSPA-LA.

Like the EPA in 40 CFR Part 197, the NRC does not provide a definitive statement of what is intended by a mean dose. However, it seems reasonable to assume that the NRC was guided by the same perspectives with respect to the concept of a mean as expressed by the EPA in Quotes (EPA8) - (EPA10). The following statement by the NRC from the supplementary information accompanying 10 CFR Parts 2, 19, 20, etc., in the Federal Register actually provides a more specific indication of what is intended by a mean than any of the statements from the EPA (Ref. [21], p. 55752):

The Commission expects that performance assessments conducted by the applicant in support of any potential license application will use probabilistic methods to simulate a wide range of 
possible future behaviors of the repository system. Each possible future behavior of the repository system is represented by a curve describing the annual dose to the RMEI as a function of time. Generally, but not necessarily, each of the possible curves is assumed to be equally likely. Because none of these possible futures can be demonstrated to describe the actual future behavior of the repository system, the Commission requires that the applicant calculate the mean of these dose versus time curves, properly weighted by their individual probabilities.

This statement specifically describes the weighting of doses by their likelihood of occurrence (i.e., a calculation involving answers to Questions (Q1), (Q2) and (Q3)). However, no specific mention is made of uncertainty in the sense of Question (Q4).

It is perhaps worth noting that the statements in Quote (NRC8) relating to the weighting of results for an individual future by the probability of that future are intuitively informative but are not correct in a literal mathematical sense. In general, individual "futures" will have a probability of zero. Thus, it does not make sense to weight the dose curve associated with an individual future by the probability of that future as indicated in Quote (NRC8). Most likely, the NRC had one, or perhaps both, of the following two situations in mind and simply did not word Quote (NRC8) explicitly. First, nonzero probabilities exist for sets of futures, not individual futures. Thus, a computational strategy to obtain a mean dose curve consistent with the apparent intent, but not the exact wording, of Quote (NRC8) is to (i) divide the possible futures into a collection of disjoint sets (i.e., the answer to Question (Q1)), (ii) determine the probability of each of these sets (i.e., the answer to Question (Q2)), (iii) select a single representative future from each set and calculate its associated dose curve (i.e., the answer to Question (Q3)), and then (iv) weight the individual dose curves by the probabilities of the associated sets. Second, in an analysis based on simple random sampling, a mean is estimated by weighting the results associated with each sample element by the reciprocal of the sample size. Thus, another computational strategy to obtain a mean dose curve consistent with the apparent intent, but not the exact wording, of Quote (NRC8) is to (i) generate a random sample from the set of all possible futures, (ii) calculate a dose curve for each sampled future, and then (iii) weight the individual dose curves by the reciprocal of the sample size. Both of the preceding approaches produce a mean dose curve in apparent consistency with the intent of Quote (NRC8). However, neither approach involves the weighting of dose curves by the probability of individual futures.

The description of a PA by the NRC in Quote (NRC2) is at a very high level. Owing to the central role played by PA in the licensing of the YM repository, it is important to examine any additional statements made by the NRC with respect to the desired characteristics of a PA carried out for this purpose. Such statements help provide guidance with respect to the conceptual and computational structure of PAs for the YM repository. Specifically, the following statement given in 10 CFR 63.102 helps provide insights with respect to what the NRC considers to be an appropriately designed and conducted PA (Ref. [21], p. 55805):

(j) Performance assessment. Demonstrating compliance with the postclosure performance objective specified at $\S 63.113$ (b) requires a performance assessment to quantitatively estimate radiological exposures to the reasonably maximally exposed individual at any time during the compliance period. The performance assessment is a systematic analysis that identifies the features, events, and processes (i.e., specific conditions or attributes of the geologic setting, degradation, deterioration, or alteration processes of engineered barriers, and interactions between the natural and engineered barriers) that might affect performance of the geologic repository; examines their effects on performance; and estimates the radiological exposures to the reasonably maximally exposed individual. The features, events, and processes considered in the performance assessment should represent a wide range of both beneficial and potentially adverse effects on performance (e.g., beneficial effects of radionuclide sorption; potentially adverse effects of fracture flow or a criticality event). Those features, events, and processes expected to materially affect compliance with $\S 63.113(\mathrm{~b})$ or be potentially adverse to performance are included, while events (event classes or scenario classes) that are very unlikely (less than one chance in 10,000 over 10,000 years) can be excluded from the analysis. An event class consists of all possible specific initiating events that are caused by a common natural process (e.g., the event class 
for seismicity includes the range of credible earthquakes for the Yucca Mountain site). Radiological exposures to the reasonably maximally exposed individual are estimated using the selected features, events, and processes, and incorporating the probability that the estimated exposures will occur.

The first three questions, (Q1) - (Q3), related to PA are clearly imbedded in the preceding description. For example, references to "events” and "event classes” involve Question (Q1): "What can happen?”; the statement "incorporating the probability that the estimated exposures will occur” involves Question (Q2): "How likely is it to happen?"; and statements such as "quantitatively estimate radiological exposures" and "examines their effects on performance” involve answering Question (Q3): "What are the consequences if it does happen?”.

The NRC's description of PA in Quote (NRC9) does not include any statement that can be identified with Question (Q4): "What is the uncertainty in the answers to the first three questions?". However, the answering of this question is clearly intended to be part of the NRC's concept of what a PA should be as indicted in the following statement (Ref. [21], p. 55807):

\section{§ 63.114 Requirements for performance assessment.}

Any performance assessment used to demonstrate compliance with § 63.113 must:

(a) Include data related to the geology, hydrology, and geochemistry (including disruptive processes and events) of the Yucca Mountain site, and the surrounding region to the extent necessary, and information on the design of the engineered barrier system used to define parameters and conceptual models used in the assessment.

(b) Account for uncertainties and variabilities in parameter values and provide for the technical basis for parameter ranges, probability distributions, or bounding values used in the performance assessment.

(c) Consider alternative conceptual models of features and processes that are consistent with available data and current scientific understanding and evaluate the effects that alternative conceptual models have on the performance of the geologic repository.

(d) Consider only events that have at least one chance in 10,000 of occurring over 10,000 years.

(e) Provide the technical basis for either inclusion or exclusion of specific features, events, and processes in the performance assessment. Specific features, events, and processes must be evaluated in detail if the magnitude and time of the resulting radiological exposures to the reasonably maximally exposed individual, or radionuclide releases to the accessible environment, would be significantly changed by their omission.

(f) Provide the technical basis for either inclusion or exclusion of degradation, deterioration, or alteration processes of engineered barriers in the performance assessment, including those processes that would adversely affect the performance of natural barriers. Degradation, deterioration, or alteration processes of engineered barriers must be evaluated in detail if the magnitude and time of the resulting radiological exposures to the reasonably maximally exposed individual, or radionuclide releases to the accessible environment, would be significantly changed by their omission.

(g) Provide the technical basis for models used in the performance assessment such as comparisons made with outputs of detailed process-level models and/or empirical observations (e.g., laboratory testing, field investigations, and natural analogs). 
Statements (b) and (c) clearly involve asking and answering Question (Q4). Further, much of the above statement involves the formulation of models used to answer Question (Q3), and statement (d) involves obtaining answers to Questions (Q1) and (Q2).

The following two statements from 10 CFR 63.101 also indicate the importance that the NRC attaches to an adequate treatment of uncertainty:

For such long-term performance, what is required is reasonable expectation, making allowance for the time period, hazards, and uncertainties involved, that the outcome will conform with the objectives for postclosure performance for the geologic repository. Demonstrating compliance will involve the use of complex predictive models that are supported by limited data from field and laboratory tests, site-specific monitoring, and natural analog studies that may be supplemented with prevalent expert judgment. Compliance demonstrations should not exclude important parameters from assessments and analyses simply because they are difficult to precisely quantify to a high degree of confidence. The performance assessments and analyses should focus upon the full range of defensible and reasonable parameter distributions rather than only upon extreme physical situations and parameter values. (Ref. [21], p. 55804)

Once again, although the criteria may be written in unqualified terms, the demonstration of compliance must take uncertainties and gaps in knowledge into account so that the Commission can make the specified finding with respect to paragraph (a)(2) of § 63.31. (Ref. [21], p. 55804)

Both the preceding statements clearly indicate that a realistic treatment of uncertainty should be a fundamental part of a PA used to support a licensing application for the YM repository and thus entail providing credible answers to Question (Q4).

In addition, a number of statements provided by the NRC as supplementary information with respect to 10 CFR Parts 2, 19, 20, etc., emphasize the importance that the NRC places on the assessment and representation of uncertainty and thus on the answering of Question (Q4):

Part 63 requires consideration of uncertainties in DOE's representation of the repository (uncertainty and variability in parameter values must be taken into account-\$63.114(b)) and the events that can happen during the compliance period (consideration of potentially disruptive events with a probability of occurrence as low as one chance in 10,000 of occurring over 10,000 years_ $§ 63.114(\mathrm{~d})$ ) to be directly included in the quantitative estimate of performance. (Ref. [21], p. 55747)

DOE is expected to conduct uncertainty analyses (i.e., evaluation of how uncertainty in parameter values affects uncertainty in the estimate of dose), including the consideration of disruptive events and associated probability of occurrence. (Ref. [21], p. 55747)

The approach defined in part 63, which requires DOE to fully address uncertainties in its performance assessment rather than requiring DOE to meet a specific level of uncertainty, is appropriate. The treatment of uncertainty in DOE's performance assessment will be an important part of NRC's review. (Ref. [21], p. 55748)

Quote (NRC13) is particularly interesting because it involves three distinct concepts related to uncertainty: (i) uncertainty in the epistemic or subjective sense related to knowledge about the appropriateness of assumptions used in an analysis, (ii) spatial variability, and (iii) uncertainty in the aleatory sense related to events that may, or may not, occur in the future. Quote (NRC14) is informative because it goes beyond asking for an analysis that simply calculates a mean outcome; in particular, it asks for an uncertainty analysis which evaluates "how uncertainty in parameter values affects uncertainty in the estimate of dose," which involves a more complex analysis than simply calculating a mean dose. Finally, Quote (NRC15) expresses the importance that the NRC places on an adequate treatment of uncertainty. 
The concepts of "event sequence" and "initiating event" are very important in organizing the underlying structure of PAs for the YM repository and are defined by the NRC as follows:

Event sequence means a series of actions and/or occurrences within the natural and engineered components of a geologic repository operations area that could potentially lead to exposure of individuals to radiation. An event sequence includes one or more initiating events and associated combinations of repository system component failures, including those produced by the action or inaction of operating personnel. (Ref. [21], p. 55793)

Initiating event means a natural or human induced event that causes an event sequence. (Ref. [21], p. 55794)

Quotes (NRC16) and (NRC17) relate to the operational phase of the repository rather than to the post-closure phase that is the focus of this presentation. However, these quotes are included because they in effect define what is known as an elementary event in a careful development of probability. In such a development and as must be the case in a PA for the YM repository, probabilities are assigned to sets of elementary events (i.e., sets of event sequences as indicated in Quote (NRC16), which in turn correspond to "event classes or scenario classes" as used in Quote (NRC9)) rather than to individual elementary events. Thus, the concept of event sequence is of fundamental importance to the analysis of both operational and post-closure performance of the YM repository. Quotes (NRC16) and (NRC17) are included from the operational requirements because, although the concept of an event sequence is equally important to the analysis of post-closure performance, similar statements defining event sequences do not appear in the post-closure requirements for the YM repository. However, as indicated by the reference to "sequences of events and processes" in Part (i) of Quote (NRC2), the concept of an event sequence is clearly intended by the NRC to be part of the conceptual structure that underlies a PA for the YM repository. For postclosure performance, the "Initiating event" of Quote (NRC17) could, for example, be an igneous or seismic occurrence taking place at a specific time and with specified properties.

This section now records a sequence of statements by the NRC that relate to the nature of occurrences (i.e., the events of the phrase "features, events and processes") that should, and should not, be included in a PA for the YM repository:

\section{§ 63.305 Required characteristics of the reference biosphere.}

(a) Features, events, and processes that describe the reference biosphere must be consistent with present knowledge of the conditions in the region surrounding the Yucca Mountain site.

(b) DOE should not project changes in society, the biosphere (other than climate), human biology, or increases or decreases of human knowledge or technology. In all analyses done to demonstrate compliance with this part, DOE must assume that all of those factors remain constant as they are at the time of submission of the license application.

(c) DOE must vary factors related to the geology, hydrology, and climate based upon cautious, but reasonable assumptions consistent with present knowledge of factors that could affect the Yucca Mountain disposal system over the next 10,000 years.

(d) Biosphere pathways must be consistent with arid or semi-arid conditions. (Ref. [21], p. 55813)

These statements are included because they relate to what is to be included in the conceptual and computational structure of a PA for the YM repository. In particular, these statements help determine the actual modeling of physical and biological processes that will take place in a PA for the YM repository. 
Finally, it is important to realize that the NRC does not view a PA in support of a licensing application for the YM repository as a risk assessment in the traditional sense of the phrase (i.e., as an analysis that is intended to calculate the actual consequences, and thus the actual risks, associated with the myriad possible future behaviors of a natural or engineered system). In particular, the dose to the RMEI is used as a means to convert potential radionuclide releases into intuitively interpretable quantities (i.e., doses). However, owing to the prescribed and stylized manner in which doses to the RMEI are defined and calculated, they do not realistically represent actual doses to individuals in the future and thus should not be viewed as the consequence component of a risk calculation. This point is emphasized by the NRC in the following statement:

However, it should be kept in mind that the performance assessment evaluates "potential" doses, not "actual" doses. For example, part 63 requires the performance assessment to assume for the next 10,000 years that the reasonably maximally exposed individual (RMEI) is a member of a community that: (1) Exists where it will intercept potential releases from the repository and (2) uses ground water but never tests the quality of this water nor treats the ground water to remove any contaminants. This specification is considered appropriately conservative for evaluating performance but most likely is not an "accurate" prediction of what will happen during the next 10,000 years (see discussion under RMEI Characteristics and Reference Biosphere for more information on the RMEI). Although the Commission does not require an "accurate" prediction of the future, uncertainty in performance estimates cannot be so large that the Commission cannot find a reasonable expectation that the postclosure performance objectives will be met (see discussion under Reasonable Expectation). (Ref. [21], p. 55748)

Thus, although a PA in support of a licensing application for the YM repository will have the same conceptual and computational structure as a risk assessment, the actual dose results cannot be given a risk-based interpretation.

\subsection{NRC Review Criteria: Yucca Mountain Review Plan (YMRP)}

Selected portions of the YMRP that specifically relate to the conceptual and computational structure of PAs for the YM repository are presented in this section. The YMRP is a large and detailed document. No attempt is made to summarize everything in it that relates to PA for the YM repository. Rather, only selected material that specifically relates to the overall structure of PAs for the YM repository is presented.

Early in the YMRP, the NRC specifically acknowledges that the DOE's license application is to be based on a detailed PA (Ref. [22], p. 2.2-1):

Risk-Informed Review Process for Performance Assessment-The performance assessment quantifies repository performance, as a means of demonstrating compliance with the postclosure performance objectives at 10 CFR 63.113. The U.S. Department of Energy performance assessment is a systematic analysis that answers the triplet risk questions: what can happen; how likely is it to happen; and what are the consequences.

(YMRP1)

The "triplet" referred to above is the classic Kaplan-Garrick ordered triple definition of risk ${ }^{32}$ that evolved out of the Reactor Safety Study ${ }^{56}$ conducted by the NRC in the mid 1970s and other reactor probabilistic risk assessments carried out in the late 1970s and early 1980s. ${ }^{57,} 58$ This risk representation also provided the conceptual and organizational basis for the NRC's reassessment of the risk from commercial nuclear power reactors carried out in the late 1980s (i.e., NUREG-1150) 59,60 and the DOE's PA in support of a successful compliance certification application to the EPA for the Waste Isolation Pilot Plant (WIPP) in the mid 1990s. ${ }^{61,} 62$ The three indicated questions have already been referred to as Questions (Q1), (Q2) and (Q3) in Sect. 2.1. As a reminder, there is also a fourth question (i.e., Q4): "What is the uncertainty in the answers to the first three questions?", or equivalently, "How much confidence do you have in the answers to the first three questions?". 
The NRC provides three acceptance criteria, with multiple subparts, to be used in reviewing the DOE's use of PA to assess compliance with the postclosure individual protection requirement (i.e., Quote (NRC1)). These criteria follow (Ref. [22], pp. 2.2-133 and 2.2-134):

Acceptance Criterion 1: Scenarios Used in the Calculation of the Annual Dose as a Function of Time Are Adequate.

(1) The annual dose as a function of time includes all scenario classes that have been determined to be sufficiently probable, or to have a sufficient effect on overall performance that they could not be screened from the total system performance assessment analyses; and

(2) The calculation of the annual dose curve appropriately sums the contribution of each of the disruptive event scenario classes. The contribution to the annual dose from each scenario class calculation properly accounts for the effects that the time of occurrence of the disruptive events comprising the scenario class has on the consequences. The annual probability of occurrence of the events used to calculate the contribution to the annual dose is consistent with the results of the scenario analysis. The probabilities of occurrence of all scenario classes, included in calculating the annual dose curve, sum to one.

(YMRP3)

Acceptance Criterion 2: An Adequate Demonstration Is Provided That the Annual Dose to the Reasonably Maximally Exposed Individual in Any Year During the Compliance Period Does Not Exceed the Exposure Standard.

(1) A sufficient number of realizations has been obtained, for each scenario class, using the total system performance assessment code, to ensure that the results of the calculations are statistically stable;

(2) The annual dose curve includes confidence intervals (e.g., 95th and 5th percentile) to represent the uncertainty in the dose calculations;

(3) Repository performance and the performance of individual components or subsystems are consistent and reasonable; and

(4) The total system performance assessment results confirm that the repository performance results in annual dose, to the reasonably maximally exposed individual, in any year, during the compliance period, that does not exceed the postclosure individual protection standard.(YMRP7)

Acceptance Criterion 3: The Total System Performance Assessment Code Provides a Credible Representation of Repository Performance.

(1) Assumptions made within the total system performance assessment code are consistent among different modules of the code. The use of assumptions and parameter values that differ among modules of the code is adequately documented;

(YMRP8)

(2) The total system performance assessment code is properly verified, such that there is confidence that the code is modeling the physical processes in the repository system in the manner that was intended. The transfer of data between modules of the code is conducted properly;

(YMRP9)

(3) The estimate of the uncertainty in the performance assessment results is consistent with the model and parameter uncertainty; and

(YMRP10) 
(4) The total system performance assessment sampling method ensures that sampled parameters have been sampled across their ranges of uncertainty.

(YMRP11)

The preceding acceptance criteria are basically describing what should be expected of any modern PA. Examples of such PAs include the NRC's reassessment of the risk from nuclear power plants ${ }^{59,} 60$ and the DOE's Compliance Certification Application for the WIPP. ${ }^{61,} 62$ 


\section{Tutorial on Probability, Uncertainty and Variability}

As clearly indicated by the material presented in Sect. 2, probability and the treatment of uncertainty are fundamental components of a PA in support of a licensing application for the YM repository. In particular, determination of expected (i.e., mean) dose to the RMEI is an inherently probabilistic calculation. For convenience and to standardize the use of certain terminology, a brief tutorial on probability and uncertainty follows.

Three basic components are involved in a formal definition of probability: a set $S$ that contains everything that could occur in the particular universe under consideration; a suitably restricted collection $\mathbb{S}$ of subsets of $S$ for which probability is defined; and a function $p$ that defines probability for the elements of $\mathbb{S}$ (Ref. [63], Section IV.3). At an intuitive level, $\mathbb{S}$ can be thought of as containing all possible subsets of $S$. However, to obtain a mathematically rigorous development of probability, $\mathbb{S}$ and $p$ must have the following properties: (i) if $\mathcal{E} \in \mathbb{S}$, then $\mathcal{E}^{\mathrm{c}} \in \mathbb{S}$, where the superscript $c$ is used to denote the complement of $\mathcal{E}$, (ii) if $\left\{\mathcal{E}_{i}\right\}$ is a countable collection of elements of $\mathbb{S}$, then $\cup_{i} \mathcal{E}_{i}$ and $\cap \mathcal{E}_{i}$ are also elements of $\mathbb{S}$, (iii) $p(S)=1$, (iv) if $\mathcal{E} \in \mathbb{S}$, then $0 \leq p(\mathcal{E}) \leq 1$, and (v) if $\{\mathcal{E}$ $\left.{ }_{i}\right\}$ is a countable collection of disjoint elements of $\mathbb{S}$ (i.e., $\mathcal{E}_{i} \cap \mathcal{E}_{j}=\varnothing$ for $i \neq j$ ), then $p\left(\cup_{i} \mathcal{E}_{i}\right)=\sum_{i} p\left(\mathcal{E}_{i}\right)$. The triple $(S, \mathbb{S}, p)$ is called a probability space and is the fundamental mathematical structure that underlies a careful development of probability. In the usual terminology of probability theory, $S$ is called the sample space; elements of $S$ are called elementary events; elements of $\mathbb{S}$ are called events; and $p$ is called a probability measure. The idea of a probability space is introduced in this presentation primarily as a notational convenience to facilitate in distinguishing between different uses of probability and in describing the calculation of expected values and distributions. The authors have neither intent nor desire to carry out a measure-theoretic development of probabilistic results.

Although the concept of a probability space is important conceptually and convenient notationally, calculations involving a probability space $(S, \mathbb{S}, p)$ are often described with a density function $d$, where

$$
p(\mathcal{E})=\int_{\mathcal{E}} d(\mathbf{x}) \mathrm{d} E
$$

for $\mathcal{E} \in \mathbb{S}, \mathbf{x} \in \mathcal{E}$, and $\mathrm{d} E$ corresponding to an increment of volume from $\mathcal{E}$. Then, the expected value, variance, cumulative distribution function (CDF), and complementary cumulative distribution function (CCDF) associated with a real-valued function $y=f(\mathbf{x})$ defined on $S$ are given by

$$
\begin{aligned}
& E(f)=\int_{S} f(\mathbf{x}) d(\mathbf{x}) \mathrm{d} S, \\
& V(y)=\int_{S}[f(\mathbf{x})-E(f)]^{2} d(\mathbf{x}) \mathrm{d} S, \\
& \operatorname{prob}(\tilde{y} \leq y)=\int_{S} \underline{\delta}_{y}[f(\mathbf{x})] d(\mathbf{x}) \mathrm{d} S,
\end{aligned}
$$

and

$$
\operatorname{prob}(\tilde{y}>y)=\int_{S} \bar{\delta}_{y}[f(\mathbf{x})] d(\mathbf{x}) \mathrm{d} S
$$

respectively, where

$$
\begin{aligned}
& \underline{\delta}_{y}[f(\mathbf{x})]= \begin{cases}1 & \text { if } f(\mathbf{x}) \leq y \\
0 & \text { otherwise }\end{cases} \\
& \bar{\delta}_{y}[f(\mathbf{x})]= \begin{cases}1 & \text { if } f(\mathbf{x})>y \\
0 & \text { otherwise }\end{cases}
\end{aligned}
$$


and $\mathrm{d} S$ represents an increment of volume from $S$. A CCDF is defined in Eq. (3.5) because of the typical usage of CCDFs rather than CDFs to represent uncertainty in risk assessments. In particular, a CCDF answers the question "How likely is it to be this bad or worse?", which is usually the question asked with respect to individual consequences in a risk assessment. However, conversion between CCDFs and CDFs is straightforward as a CDF is simply one minus the corresponding CCDF.

The expected value in Eq. (3.2) is of central importance in the EPA's and the NRC's regulations for the YM repository, where $y$ corresponds to dose to the RMEI at a particular time. Specifically, because the expected (i.e., mean) value of a function is defined by an integral, any calculation of expected dose to the RMEI as specified in Quotes (EPA7) and (NRC7) must in some way involve the evaluation of an integral of the form indicated in Eq. (3.2).

The representation and incorporation of uncertainty figures prominently in the EPA's and NRC's standards for the YM repository. For example, see references to uncertainty in Quotes (EPA2), (EPA3) and (EPA5) - (EPA12) from the EPA and in Quotes (NRC2), (NRC3) and (NRC5) - (NRC13) from the NRC. In addition, Quotes (YMRP1) - (YMRP5), (YMRP10) and (YMRP11) from the NRC's YMRP also relate to the importance attached to the representation of uncertainty. Examination of the indicated statements shows that three different concepts of uncertainty are involved in PA for the YM repository: (i) uncertainty about what will happen in the future, (ii) uncertainty about parameters, models, and other analysis assumptions, and (iii) variability.

Uncertainty about what will happen in the future can be seen in statements such as (i) "weighted by their probability of occurrence" in Quotes (EPA2) and (NRC2), (ii) "Because none of these possible futures can be demonstrated to describe the actual future behavior of the repository system, the Commission requires that the applicant calculate the mean of these dose curves, properly weighted by their individual probabilities” in Quote (NRC6), and (iii) "The probabilities of occurrence of all scenario classes, included in calculating the annual dose curve, sum to one" in Quote (YMRP3). Uncertainty of the type indicated in the preceding statements about events whose future occurrence is assumed to be random, at least insofar as our ability to forecast such occurrences is concerned, is called aleatory uncertainty. ${ }^{23-25}$ Other descriptors sometimes used in the designation of aleatory uncertainty include stochastic, type A and irreducible. The preceding statements indicate that the EPA and the NRC intend for probability to be used in the mathematical characterization of aleatory uncertainty. Thus, underlying PAs for the YM repository, there must be a probability space $\left(\mathcal{A}, \mathbb{A}, p_{A}\right)$ for aleatory uncertainty. The general nature of the probability space $\left(\mathcal{A}, \mathbb{A}, p_{A}\right)$ is discussed in more detail in Sect. 5 .

Uncertainty about parameters, models and other analysis assumptions can be seen in statements such as (i) "focuses performance assessments and analyses upon the full range of defensible and reasonable parameter distributions" in Quotes (EPA3) and (NRC3), (ii) "Account for uncertainties and variabilities in parameter values and provide for the technical basis for parameter ranges, probability distributions, or bounding values used in the performance assessment" in Quote (NRC8), (iii) "performance assessments and analyses should focus on the full range of defensible and reasonable parameter distributions" in Quote (NRC9), and (iv) "The estimate of the uncertainty in the performance assessment results is consistent with the model and parameter uncertainty" in Quote (YMRP10). Uncertainty of the type indicated in the preceding statements derives from a lack of knowledge about a quantity, a model, or an assumption assumed to have a fixed value in the context of a specific analysis and is usually referred to as epistemic uncertainty. ${ }^{23-25}$ Other descriptors sometimes used in the designation of epistemic uncertainty include subjective, state of knowledge, type B and reducible. The preceding statements indicate that the EPA and the NRC intend for probability to be used in the mathematical characterization of epistemic uncertainty. Thus, underlying the PAs for the YM repository, there must be a probability space $\left(\mathcal{E}, \mathbb{E}, p_{E}\right)$ for epistemic uncertainty. The general nature of the probability space $\left(\mathcal{E}, \mathbb{E}, p_{E}\right)$ is discussed in more detail in Sect. 6. As an aside, it is interesting to note that there is evidence that individuals process information related to aleatory uncertainty differently from information related to epistemic uncertainty. ${ }^{64}$

The parameter distributions that give rise to a probability space $\left(\mathcal{E}, \mathbb{E}, p_{E}\right)$ used to represent epistemic uncertainty are usually developed through some form of expert review process. ${ }^{65-76}$ The purpose of this review process is to assemble available information about parameters into a mathematical structure that can be incorporated into subsequent analyses. The extent of this review process can vary widely depending on the purpose of the analysis, the size of the analysis, and the resources available to carry out the analysis. At one extreme is a relatively small study in which a 
single analyst both develops the uncertainty characterizations (e.g., on the basis of personal knowledge or a cursory literature review) and carries out the analysis. At the other extreme is a large analysis on which important societal decisions will be based and for which uncertainty characterizations are carried out for a large number of variables by teams of outside experts who support the analysts actually performing the analysis. Examples of very extensive expert review processes carried out to characterize epistemic uncertainty are provided by the NRC's reassessment of reactor risk (i.e.,

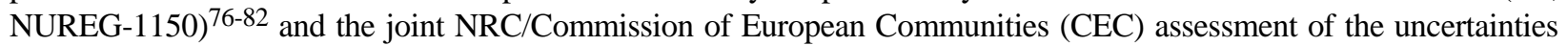
associated with parameters used in reactor accident consequence models. ${ }^{83-87}$

Although other mathematical structures for the representation of epistemic uncertainty exist (e.g., interval analysis, fuzzy set theory, possibility theory, evidence theory), ${ }^{88-97}$ the NRC has clearly indicated an intent that probability be used to characterize epistemic uncertainty in PAs for the YM repository (e.g., see Quotes (NRC2), (NRC3), (NRC7), (NRC10), (NRC11)).

In developing the parameter distributions that give rise to the probability space $\left(\mathcal{E}, \mathbb{E}, p_{E}\right)$, the goal is to develop distributions that provide an unbiased characterization of the uncertainty with respect to the appropriate value to use for each parameter under consideration. In particular, such distributions should provide uncertainty characterizations that are neither deliberately pessimistic (i.e., conservative) nor deliberately optimistic (i.e., nonconservative). As indicated in statements quoted in Sects. 2.1 and 2.2, both the EPA and the NRC desire a realistic rather than a conservative PA for the YM repository. As examples, the statements "If choices are made that make the simulations very unrealistic, the confidence that can be placed in the modeling results is very limited." and "Overly conservative assumptions made in developing performance scenarios can bias the analyses in the direction of unrealistically extreme situations, which in reality may be highly improbable, and can deflect attention from questions critical to developing an adequate understanding of the expected features, events and processes.” appear in Quote (EPA13), and the statement "The performance assessments and analyses should focus upon the full range of defensible and reasonable parameter distributions rather than only upon extreme physical situations and parameter values" appears in Quote (NRC11). The indicated statements clearly indicate a desire for a PA that has not been biased by conservative assumptions. The importance of avoiding conservative analyses has been emphasized by a number of individuals, including a chairman of the NRC. ${ }^{98-102}$

Ideally, if deliberate conservatism is to be included in a PA for the YM repository, this conservatism should be incorporated after a nonconservative PA has been performed. With this approach, the shifting of the results of the PA by the addition of one or more conservative assumptions can be clearly identified and understood. Such an analysis could be of benefit in making a convincing argument for the safety of the YM repository. However, without first carrying out an unbiased PA, there is no way to meaningfully assess the effects, and hence the potential benefits and detriments, of skewing PA results through the imposition of conservative assumptions. As an example, this approach underlies the compliance certification for the WIPP, where an initial PA was carried out by the $\mathrm{DOE}^{61,62}$ and then a modification of this PA was carried out with changes specifically requested by the EPA. ${ }^{103}$

Recognition of the potential importance of variability in assessing the compliance of the YM facility with applicable standards is demonstrated by references to "variability" in Quotes (NRC8) and (NRC11). In the two indicated quotes, variability appears in conjunction with uncertainty in the statements "Account for uncertainties and variabilities in parameter values" in Quote (NRC8) and "uncertainty and variability in parameter values must be taken into account” in Quote (NRC11). However, uncertainty in the sense of epistemic uncertainty and variability in the sense of a quantity having different values as a function of space and/or time are very different concepts. In particular, epistemic uncertainty involves a lack of knowledge about the appropriate value to use for a quantity that is assumed to have a fixed value in the context of a particular analysis. Probability provides the mathematical structure that is used in most analyses to characterize such uncertainty. In contrast, variability in a quantity is characterized as a function of space and/or time (e.g., $f(x, y), f(x, y, z), f(x, y, z, t), f(t), \ldots$, where $x, y$ and $z$ correspond to spatial coordinates and $t$ corresponds to time). For some quantities, functions of the form just indicated will likely be used as input to PAs for the YM repository; for other quantities, spatial and/or temporal variability will likely be reduced to an expected value over space and/or time, and then this expected value used as input to a PA.

An important point to recognize is that there is almost always epistemic uncertainty in the characterization of variability. For example, if a function $f(x, y)$ characterizes the two-dimensional variability of some quantity, there 
are most likely many possible values for this function of varying levels of credibility. Thus, the function $f(x, y)$ characterizes spatial variability, but a lack of knowledge with respect to how to exactly define $f(x, y)$ is epistemic uncertainty. Similarly, there can be, and almost always is, epistemic uncertainty with respect to the values of quantities used in the characterization of aleatory uncertainty. For example, the occurrence of a certain process (e.g., volcanism) might be assumed to follow a Poisson process with a rate constant $\lambda$ (units: $\mathrm{yr}^{-1}$ ). The rate constant $\lambda$ characterizes aleatory uncertainty and gives rise to probabilities of volcanic events occurring over time intervals of different lengths. However, the inability to confidently assign an exact value for $\lambda$ is epistemic uncertainty.

It is worthwhile to briefly consider the phrases "features, events, and processes, and sequences of events and processes" and "features, events and processes" that appear often in the EPA and NRC standards for the YM repository (e.g., see Quotes (EPA2), (NRC2), (NRC7), (NRC8), (NRC16)). As suggested by its name, a feature is a largescale property of the system under consideration (e.g., a fault, a geologic formation, an aquifer, ...). A feature either exists or it does not exist. There can be epistemic uncertainty about whether or not a feature exists (i.e., maybe it is there or maybe it is not there) and also epistemic uncertainty about the properties of a feature (e.g., permeability, porosity, fracture spacing, thermal conductivity, ...). Often, there is spatial variability in the properties of a feature. There can also be epistemic uncertainty in how to define such variability. However, as previously discussed, variability and epistemic uncertainty are distinct concepts. An event is something that occurs over a period of time that is short relative to the period of analysis (e.g., igneous or seismic occurrences); in essence, an event is a discontinuity in the behavior of the system that occurs at a specific point in time. The occurrence, or nonoccurrence, of events is considered to be aleatory uncertainty and is distinct from epistemic uncertainty. However, there can be epistemic uncertainty in quantities used to characterize aleatory uncertainty (e.g., means, standard deviations, occurrence rates, ...). The reference to "sequence of events" derives from the fact that the occurrence of one or more aleatory events is always considered over some period of time (e.g., 0 to 10,000 yr in parts of the regulations for the YM repository); such sequences of events correspond to elementary events associated with the probability space for aleatory uncertainty. A process is something that takes place continuously over a period of time that is long relative to the period of analysis (e.g., groundwater flow, heat flow, corrosion, ...). Although a process may be, but is not necessarily, initiated by a specific aleatory event, it is usually represented by a model (e.g., a system of ordinary or partial differential equations) that predicts the time-dependent, and often spatially dependent, behavior of the process. Often, there is substantial epistemic uncertainty associated with the model inputs used in modeling complex processes (e.g., dual porosity fluid flow and solute transport in a fractured geologic medium) and there can also be epistemic uncertainty with respect to the choice of a model itself (i.e., model uncertainty as it is sometimes called, e.g., see Ref [104]).

The dual use of probability to represent both aleatory uncertainty and epistemic uncertainty, as is specified by both the EPA and the NRC for use in PAs for the YM repository, can be traced back to the beginnings of the formal development of probability theory in the late sixteen hundreds. ${ }^{7,105-107}$ The use of probability in PAs for complex systems is a topic of wide interest and many references are available that provide additional information and perspectives on this use. ${ }^{23,}$, 25-29, $31,108-110$ 


\section{Properties of Poisson Processes}

At least two types of disruptions must be considered in PAs for the proposed YM repository: igneous events and seismic events. Both of these disruptions are assumed to have a pattern of occurrence that can be represented as a Poisson process. Because the properties of Poisson processes will play an important role in determining expected dose to the RMEI in PAs for the YM repository, it is useful to briefly review these properties at the beginning of this presentation. Then, these properties can be referred to as needed in the subsequent derivation and calculation of expected dose to the RMEI.

The descriptor Poisson process is used to designate a set of potentially realizable sequences of occurrences (e.g., sequences of seismic events occurring at different times in the future) with certain special probabilistic characteristics. For the purposes of this presentation, a Poisson process can be described in terms of a function $N(r, s)$ defined for $0 \leq r<s<\infty$, where

$$
N(r, s)=\text { number of occurrences in the time interval }[r, s] \text {. }
$$

Specifically, the potential sequences of occurrences that give rise to different possible values for $N(r, s)$ are said to follow a (stationary or homogeneous) Poisson process provided

$$
\begin{aligned}
& \operatorname{prob}[N(r, r+\Delta t)=1]=\lambda \Delta t+o(\Delta t), \\
& \operatorname{prob}[N(r, r+\Delta t) \geq 2]=o(\Delta t)
\end{aligned}
$$

and

$$
\operatorname{prob}[N(r, s)=k \text { and } N(u, v)=l \mid[r, s] \cap[u, v]=\varnothing]=\operatorname{prob}[N(r, s)=k] \operatorname{prob}[N(u, v)=l],
$$

where (i) prob denotes probability and the associated vertical line indicates conditionality, (ii) $\lambda$ is independent of $r$, and (iii) the $o(\Delta t)$ notation is an abbreviation for

$$
\lim _{\Delta t \rightarrow 0^{+}} \operatorname{prob}[N(r, r+\Delta t)=1] / \Delta t=\lambda
$$

and

$$
\lim _{\Delta t \rightarrow 0^{+}} \operatorname{prob}[N(r, r+\Delta t) \geq 2] / \Delta t=0
$$

in Eqs. (4.2) an (4.3), respectively.

The conditions in Eqs. (4.2) and (4.3) require that, in a small interval of length $\Delta t$, the probability of one event occurring is approximately $\lambda \Delta t$ and the probability of two or more events occurring is approximately zero. The condition in Eq. (4.4) requires independence for the numbers of events occurring in two disjoint intervals. The quantity $\lambda$ is the defining rate for the process and has units of inverse time (e.g., $\mathrm{yr}^{-1}$ ).

The following important properties hold for the Poisson process defined by the conditions in Eqs. (4.2) - (4.4):

$$
\begin{aligned}
& \operatorname{prob}[N(r, s)=0]=\exp [-\lambda(s-r)], \\
& \operatorname{prob}[N(r, s)=1]=\lambda(s-r) \exp [-\lambda(s-r)],
\end{aligned}
$$




$$
\operatorname{prob}[N(r, s) \geq 1]=1-\operatorname{prob}[N(r, s)=0]=1-\exp [-\lambda(s-r)]
$$

and, in general,

$$
\operatorname{prob}[N(r, s)=k]=\left\{[\lambda(s-r)]^{k} / k !\right\} \exp [-\lambda(s-r)]
$$

for $k=0,1,2, \ldots$ Further,

$$
E[N(r, s)]=\lambda(s-r)
$$

where $E$ denotes expected value. When $\lambda(s-r)$ is small (i.e., much less than one), then $\lambda(s-r)$ approximates $\operatorname{prob}[N(r, s)=1]$; however, $\lambda(s-r)$ ceases to be a valid approximation to $\operatorname{prob}[N(r, s)=1]$ as it increases in size.

An important property of stationary Poisson processes is that, if the process occurs exactly one time in the interval $[r, s]$ (i.e., if $N(r, s)=1$ ), then the occurrence time has a uniform distribution on $[r, s]$. Specifically,

$$
d[t \mid N(r, s)=1]=1 /(s-r)
$$

is the density function defined on $[r, s]$ for time of occurrence.

A new Poisson process with rate

$$
\lambda=\lambda_{1}+\lambda_{2}+\ldots+\lambda_{n}
$$

can be created by combining $n$ Poisson processes with rates $\lambda_{1}, \lambda_{2}, \ldots, \lambda_{n}$ provided the occurrences across the rates are independent (i.e., the occurrence of an event associated with process $i$ has no effect on the potential occurrence of an event associated with process $j$ and vice versa). Then, $\lambda$ is the occurrence rate for the process that results when no distinction is made between occurrences associated with the processes characterized by $\lambda_{1}, \lambda_{2}, \ldots, \lambda_{n}$.

The Poisson process defined by the conditions in Eqs. (4.2) - (4.4) is referred to as a stationary or homogeneous Poisson to emphasize that the value for $\lambda$ is constant. A generalization is to replace the condition in Eq. (4.2) with

$$
\operatorname{prob}[N(r, r+\Delta t)=1]=\lambda(r) \Delta t+o(\Delta t)
$$

With this formulation, $\lambda(r)$ is now a function of time, and the resultant Poisson process is referred to as a nonstationary or nonhomogeneous Poisson process to emphasize that $\lambda$ is no longer constant with time. For example, it has been proposed that the occurrence of igneous events in the vicinity of the YM repository can be characterized by a nonstationary Poisson process. ${ }^{111}$

When nonstationary Poisson processes are under consideration, the relationships in Eqs. (4.7) - (4.13) have similar but slightly more complicated forms. Specifically,

$$
\begin{aligned}
& \operatorname{prob}[N(r, s)=0]=\exp \left[-\int_{r}^{s} \lambda(t) d t\right] \\
& \operatorname{prob}[N(r, s)=1]=\left[\int_{r}^{s} \lambda(t) d t\right] \exp \left[-\int_{r}^{s} \lambda(t) d t\right], \\
& \operatorname{prob}[N(r, s) \geq 1]=1-\exp \left[-\int_{r}^{s} \lambda(t) d t\right]
\end{aligned}
$$




$$
\begin{aligned}
& \operatorname{prob}[N(r, s)=k]=\left\{\left[\int_{r}^{s} \lambda(t) d t\right]^{k} / k !\right\} \exp \left[-\int_{r}^{s} \lambda(t)\right] d t, \\
& E[N(r, s)]=\int_{r}^{s} \lambda(t) d t
\end{aligned}
$$

and

$$
\lambda(t)=\lambda_{1}(t)+\lambda_{2}(t)+\ldots+\lambda_{n}(t)
$$

Further, if a nonstationary Poisson process with rate $\lambda(t)$ occurs exactly one time in the interval $[r, s]$, then

$$
d[t \mid N(r, s)=1]=\lambda(t) / \int_{r}^{s} \lambda(\tau) d \tau
$$

is the density function defined on $[r, s]$ for time of occurrence. The results in Eqs. (4.14) - (4.21) reduce to those in Eqs. (4.7) - (4.13) when the time-dependent $\lambda$ 's are replaced by constant values.

Additional introductory information on Poisson processes is available in the text by Ross (Ref. [112], Chapt. 5). More advanced treatments are also available. ${ }^{113-116}$ 
This page intentionally left blank. 


\section{Expected Dose Without Epistemic Uncertainty}

An intuitive development of expected dose to the RMEI without the inclusion of epistemic uncertainty follows. The incorporation of epistemic uncertainty into expected dose to the RMEI is then described in the next section (Sect. 6). The presentation begins with the consideration of a single class of disruptive events (e.g., igneous events) whose occurrence through time is characterized by a Poisson process with a rate constant $\lambda_{D}\left(\mathrm{yr}^{-1}\right)$. Each individual event is defined by a time $t$ (yr) of occurrence and a vector $\mathbf{p}$ of additional properties (e.g., number of waste packages contacted by intruding magma, whether or not a surface eruption occurs, ... if igneous events are under consideration). Further, a time interval $[a, b]$ is under consideration (e.g., $[a, b]=[0,10,000 \mathrm{yr}]$, where time $0 \mathrm{yr}$ corresponds to closure of the repository). In general, any number of events could occur over the time interval $[a, b]$, although the probability of various numbers of occurrences will depend on the size of $\lambda_{D}$ relative to the length of [a, $b]$ (i.e., on the value of the product $\lambda_{D}(b-a)$; see Eq. (4.10)).

For notational convenience, let

$$
\mathbf{a}=\left[n, t_{1}, \mathbf{p}_{1}, t_{2}, \mathbf{p}_{2}, \ldots, t_{n}, \mathbf{p}_{n}\right]
$$

denote a sequence of $n$ occurrences at times $t_{1} \leq t_{2} \leq \ldots \leq t_{n}$ and associated property vectors $\mathbf{p}_{1}, \mathbf{p}_{2}, \ldots, \mathbf{p}_{n}$. Then,

$$
\mathcal{A}(a, b)=\left\{\mathbf{a}: \mathbf{a}=\left[n, t_{1}, \mathbf{p}_{1}, t_{2}, \mathbf{p}_{2}, \ldots, t_{n}, \mathbf{p}_{n}\right] \text { for } n=0,1,2, \ldots \text { and } a \leq t_{1} \leq t_{2} \leq \ldots \leq t_{n} \leq b\right\}
$$

represents the set of all sequences of occurrences (i.e., futures) over the time interval $[a, b]$, with

$$
\mathbf{a}=\mathbf{a}_{N}(a, b)=[0]
$$

understood to represent the future with no occurrence of the disruption under consideration over the time interval $[a, b]$ (i.e., the nominal future). The simpler representations

$$
\mathbf{a}=\left[n, t_{1}, t_{2}, \ldots, t_{n}\right]
$$

and

$$
\mathbf{a}=\left[n, t_{1}, p_{1}, t_{2}, p_{2}, \ldots, t_{n}, p_{n}\right]
$$

for $\mathbf{a}$, and thus correspondingly for $\mathcal{A}(a, b)$, result when the disruption is defined by time of occurrence only and time of occurrence and a single property, respectively.

Given that only the single indicated disruption is under consideration, $\mathcal{A}(a, b)$ represents the set of all possible futures for the time interval $[a, b]$. Thus, in standard terminology from probability theory, $\mathcal{A}(a, b)$ would be the sample space for sequences of occurrences over the time interval $[a, b]$, and each sequence $\mathbf{a}$ of occurrences in $\mathcal{A}(a$, $b$ ) would be an elementary event. Although never expressly identified, the existence of sets analogous to $\mathcal{A}(a, b)$ is alluded to by the NRC in the statements such as "Identifies... sequences of events and processes" (see Quote (NRC2)) and "wide range of possible future behaviors" (see Quote (NRC7)). Further, the existence of sets of the form $\mathcal{A}(a, b)$ is fundamental to the determination of probabilities (see Quotes (NRC2), (NRC6), (NRC9), (NRC10), (NRC13), (YMRP2), (YMRP3)) and expected (i.e., mean) dose (see Quotes (NRC7), (NRC8), (YMRP2), (YMRP3)) specified by the NRC.

Probabilities are defined for subsets of $\mathcal{A}(a, b)$. Of particular interest are the subsets

$$
\mathcal{A}_{n}(a, b)=\left\{\mathbf{a}: \mathbf{a}=\left[n, t_{1}, \mathbf{p}_{1}, t_{2}, \mathbf{p}_{2}, \ldots, t_{n}, \mathbf{p}_{n}\right], a \leq t_{1} \leq t_{2} \leq \ldots \leq t_{n} \leq b\right\}
$$


for $n=0,1,2, \ldots$, with each set $\mathcal{A}_{n}(a, b)$ containing all futures in which exactly $n$ occurrences take place over the time interval $[a, b]$. In terminology used by the NRC, each set $\mathcal{A}_{n}(a, b)$ is a scenario class (or, sometimes, an event or event class; see Quotes (NRC9), (NRC10d), (YMRP2), (YMRP3), (YMRP4)) and corresponds to what is usually called an event in standard terminology from probability theory. More generally, any subset of $\mathcal{A}(a, b)$ corresponds to what the NRC refers to as a scenario class. The probability $p_{A}\left[\mathcal{A}_{n}(a, b)\right]$ of $\mathcal{A}_{n}(a, b)$ is given by

$$
p_{A}\left[\mathcal{A}_{n}(a, b)\right]=\left\{\left[\lambda_{D}(b-a)\right]^{n} / n !\right\} \exp \left[-\lambda_{D}(b-a)\right]
$$

as indicated in Eq. (4.10). The subscript $A$ appears in $p_{A}\left[\mathcal{A}_{n}(a, b)\right]$ to emphasize that this probability derives from aleatory uncertainty.

A probability space $\left(\mathcal{A}, \mathbb{A}, p_{A}\right)$ of the form indicated in Sect. 3 characterizing aleatory uncertainty has now effectively been introduced. The sample space $\mathcal{A}$ is the set $\mathcal{A}(a, b)$ defined in Eq. (5.2); the elementary events are the elements $\mathbf{a}$ of $\mathcal{A}(a, b)$ defined in Eq. (5.1); the events contained in $\mathbb{A}=\mathbb{A}(a, b)$ are subsets of $\mathcal{A}(a, b)$ as exemplified by the sets $\mathcal{A}_{n}(a, b)$ defined in Eq. (5.6); and the probability measure $p_{A}$ is defined for elements of $\mathbb{A}(a, b)$ as exemplified by the defining relationship for $p_{A}\left[\mathcal{A}_{n}(a, b)\right]$ in Eq. (5.7). In the preceding, the descriptor "exemplified" is used in conjunction with the sets $\mathcal{A}_{n}(a, b)$ and the probabilities $p_{A}\left[\mathcal{A}_{n}(a, b)\right]$ because, in general, $\mathbb{A}(a, b)$ will contain many more sets than just the sets $\mathcal{A}_{n}(a, b)$ (i.e., intuitively, but not in a formally correct sense, all subsets of $\mathcal{A}(a, b)$ ) and the probability $p_{A}(\mathcal{E})$ for an arbitrary element $\mathcal{E}$ of $\mathbb{A}(a, b)$ can involve a more complex definition than shown in Eq. (5.7) (e.g., the probability of $\mathcal{E}$ can be affected by restrictions in the definition of $\mathcal{E}$ involving the elements of the property vector $\mathbf{p}$ and by the probability distributions assigned to these elements). In most analyses, $p_{A}$ is implicitly defined by assigning distributions to the individual elements of the vector $\mathbf{a}$, and probabilities are actually defined and calculated for only a limited number of subsets of the sample space $\mathcal{A}$ that have special significance to the analysis.

Each element $\mathbf{a}$ of $\mathcal{A}(a, b)$ gives rise to a time dependent dose $D(\tau \mid \mathbf{a})$ to the RMEI, where

$$
D(\tau \mid \mathbf{a})=\text { dose (mrem/yr) to the RMEI at time } \tau(\mathrm{yr}) \text { associated with the element (i.e., future) } \mathbf{a} \text { of } \mathcal{A}(a, b) \text {. }
$$

The NRC regulations for the YM repository specify a bound of $15 \mathrm{mrem} / \mathrm{yr}$ on the expected (i.e., mean) value for $D(\tau \mid \mathbf{a})$ (see Quotes (NRC7), (NRC8), (YMRP2), (YMRP3)). In the example of this section, the required expected value derives from the possible values for $\mathbf{a}$ and the "likelihood" of these values. In an actual PA for the YM repository, $D(\tau \mid \mathbf{a})$ would be a very complicated function involving the representation of many physical processes (e.g., fluid flow, heat flow, waste package degradation, radionuclide transport, ...); however, this level of detail in the definition of $D(\tau \mid \mathbf{a})$ is not needed in this presentation and would actually be a distraction that obscured the basic conceptual ideas being described. The necessity of using computational models symbolically represented by $D(\tau \mid \mathbf{a})$ to estimate dose to the RMEI is recognized by the NRC (e.g., see Quotes (NRC9), (NRC10), (NRC11)).

With respect to terminology, the mean dose to the RMEI referred to by the NRC is what most English language texts on probability refer to as the expected value of a random variable and designate with a capital $E$ as done in Eq. (3.2) (e.g., see, at increasing levels of sophistication, Chapt. 2, Ref. [112], Chapts. 7 and 8, Ref. [117], and Sect. IV.4, Ref. [63]). As a reminder, a random variable is a function defined on the sample space $\mathcal{A}$ associated with a probability space such as $\left(\mathcal{A}, \mathbb{A}, p_{A}\right)$ indicated above. In the context of dose to the RMEI in the NRC's regulations for the YM repository, the primary random variable under consideration is a function of the form $D(\tau \mid \mathbf{a})$ introduced in Eq. (5.8) for elements $\mathbf{a}$ of the sample space $\mathcal{A}(a, b)$. For consistency with standard usage, this presentation refers to expected dose to the RMEI rather than mean dose to the RMEI and uses a capital $E$ as the designator for the expectation operator.

In concept, the expected value $E_{A}[D(\tau \mid \mathbf{a})]$ for $D(\tau \mid \mathbf{a})$ can be approximated by generating a sequence

$$
\mathbf{a}_{i}, i=1,2, \ldots, n S \text {, }
$$


of random samples from $\mathcal{A}(a, b)$ in consistency with the distributions assigned to the individual elements of $\mathbf{a}$ and then estimating $E_{A}[D(\tau \mid \mathbf{a})]$ by

$$
E_{A}[D(\tau \mid \mathbf{a})] \cong \sum_{i=1}^{n S} D\left(\tau \mid \mathbf{a}_{i}\right) / n S .
$$

As for $p_{A}\left[\mathcal{A}_{n}(a, b)\right]$, the subscript $A$ appears in $E_{A}[D(\tau \mid \mathbf{a})]$ to emphasize that this expected value derives from aleatory uncertainty.

Estimating $E_{A}[D(\tau \mid \mathbf{a})]$ by random sampling directly from $\mathcal{A}(a, b)$ as indicated in Eq. (5.9) is conceptually straightforward and thus very appealing. Unfortunately, this approach does not provide a computationally effective way to estimate $E_{A}[D(\tau \mid \mathbf{a})]$ because of the very large sample sizes required to assure adequate representation of the elements of $\mathcal{A}(a, b)$ that involve the occurrence of one or more disruptions. For example, if $\lambda_{D}=10^{-7} \mathrm{yr}^{-1}$ and $[a, b]=[0,10,000 \mathrm{yr}]$, then

$$
\begin{aligned}
& p_{A}\left[\mathcal{A}_{0}(a, b)\right]=\left\{\left[10^{-7}\left(10^{4}-0\right)\right]^{0} / 0 !\right\} \exp \left[-10^{-7}\left(10^{4}-0\right)\right] \cong 9.99 \times 10^{-1}, \\
& p_{A}\left[\mathcal{A}_{1}(a, b)\right]=\left\{\left[10^{-7}\left(10^{4}-0\right)\right]^{1} / 1 !\right\} \exp \left[-10^{-7}\left(10^{4}-0\right)\right] \cong 9.99 \times 10^{-4}, \\
& p_{A}\left[\mathcal{A}_{2}(a, b)\right]=\left\{\left[10^{-7}\left(10^{4}-0\right)\right]^{2} / 2 !\right\} \exp \left[-10^{-7}\left(10^{4}-0\right)\right] \cong 5.00 \times 10^{-7},
\end{aligned}
$$

and, as a result, a random sample from $\mathcal{A}(a, b)$ of size $n S=100,000$ would have approximately 99,900 sample elements that involved no disruption, approximately 100 sample elements that involved the occurrence of exactly one disruption, and most likely no elements that involved the occurrence of two or more disruptions. Because of this sparse coverage of potential disruptions even for very large sample sizes, random sampling directly from $A(a, b)$ is not an effective numerical procedure for the estimation of $E_{A}[D(\tau \mid \mathbf{a})]$.

As recognized by the NRC, a solution to obtaining an adequate coverage of $\mathcal{A}(a, b)$ in the estimation of $E_{A}[D(\tau \mid \mathbf{a})]$ is to (i) divide $\mathcal{A}(a, b)$ into disjoint subsets (i.e., scenario classes), (ii) estimate expected doses for these sets individually, and then (iii) estimate $E_{A}[D(\tau \mid \mathbf{a})]$ from the expected doses for the individual sets and the probabilities for these sets (e.g., see Quote (YMRP1)). This estimation procedure for $E_{A}[D(\tau \mid \mathbf{a})]$ can be formally represented by

$$
\begin{aligned}
E_{A}[D(\tau \mid \mathbf{a})] & =\int_{\mathcal{A}(a, b)} D(\tau \mid \mathbf{a}) d_{A}(\mathbf{a}) \mathrm{d} A \\
& =\int_{\cup \mathcal{A}_{n}} D(\tau \mid \mathbf{a}) d_{A}(\mathbf{a}) \mathrm{d} A \\
& =\sum_{n} \int_{\mathcal{A}_{n}} D(\tau \mid \mathbf{a}) d_{A}(\mathbf{a}) \mathrm{d} A \\
& =\sum_{n}\left\{\int_{\mathcal{A}_{n}} D(\tau \mid \mathbf{a})\left[d_{A}(\mathbf{a}) / \int_{\mathcal{A}_{n}} d_{A}(\mathbf{a}) \mathrm{d} A\right] \mathrm{d} A\right\}\left\{\int_{\mathcal{A}_{n}} d_{A}(\mathbf{a}) \mathrm{d} A\right\} \\
& =\sum_{n} E_{A}\left[D\left(\tau \mid \mathbf{a} \in \mathcal{A}_{n}\right)\right] p_{A}\left(\mathcal{A}_{n}\right),
\end{aligned}
$$


where (i) $\mathcal{A}_{1}, \mathcal{A}_{2}, \ldots$, is a sequence of sets satisfying $\mathcal{A}(a, b)=\cup_{n} \mathcal{A}_{n}$ and $\mathcal{A}_{i} \cap \mathcal{A}_{j}=\varnothing$ for $i \neq j$ (e.g., sets of the form defined in Eq. (5.6)), (ii) $d_{A}(\mathbf{a})$ is the density function associated with the probability space for $\mathbf{a}$ and

$$
p_{A}\left(\mathcal{A}_{n}\right)=\int_{\mathcal{A}_{n}} d_{A}(\mathbf{a}) \mathrm{d} A \neq 0
$$

is the probability of the set $\mathcal{A}_{n}$, and (iii) $E_{A}\left[D\left(\tau \mid \mathbf{a} \in \mathcal{A}_{n}\right)\right]$ is the expected value of $D(\tau \mid \mathbf{a})$ conditional on $\mathbf{a} \in \mathcal{A}_{n}$.

As an example,

$$
\begin{aligned}
E_{A}[D(\tau \mid \mathbf{a})] & =\sum_{n=0}^{\infty} E_{A}\left\{D\left[\tau \mid \mathbf{a} \in \mathcal{A}_{n}(a, b)\right]\right\} p_{A}\left[\mathcal{A}_{n}(a, b)\right] \\
& \cong \sum_{n=0}^{\infty} \hat{E}_{A}\left\{D\left[\tau \mid \mathbf{a} \in \mathcal{A}_{n}(a, b)\right]\right\} p_{A}\left[\mathcal{A}_{n}(a, b)\right],
\end{aligned}
$$

where

$$
E_{A}\left\{D\left[\tau \mid \mathbf{a} \in \mathcal{A}_{n}(a, b)\right]\right\}=\begin{aligned}
& \text { expected dose (mrem/yr) to the RMEI at time } t \text { (yr) conditional on the } \\
& \text { occurrence of scenario class } \mathcal{A}_{n}(a, b)
\end{aligned}
$$

and $\hat{E}_{A}\left\{D\left[\tau \mid \mathbf{a} \in \mathcal{A}_{n}(a, b)\right]\right\}$ is an estimate of $E_{A}\left\{D\left[\tau \mid \mathbf{a} \in \mathcal{A}_{n}(a, b)\right]\right\}$ obtained in some appropriate manner (e.g., by random sampling from $\mathcal{A}_{n}(a, b)$ ). The expected dose $E_{A}\left\{D\left[\tau \mid \mathbf{a} \in \mathcal{A}_{n}(a, b)\right]\right\}$ is conditional in the sense that it is calculated with the assumption that $\mathcal{A}_{n}(a, b)$ has occurred; the actual probability for $\mathcal{A}_{n}(a, b)$ enters into the estimation of $E_{A}[D(\tau \mid \mathbf{a})]$ through the factor $p_{A}\left[\mathcal{A}_{n}(a, b)\right]$ in Eq. (5.16). In the context of the triplet risk questions indicated in Questions (Q1) - (Q3) and again in Quote (YMRP1), the sets $\mathcal{A}_{n}(a, b)$ are the answer to the question "What can happen?," the probabilities $p_{A}\left[\mathcal{A}_{n}(a, b)\right]$ are the answer to the question "How likely is it to happen?", and the expected doses $E_{A}\left\{D\left[\tau \mid \mathbf{a} \in \mathcal{A}_{n}(a, b)\right]\right\}$ are the answer to the question "What are the consequences?".

Although formally correct, the approximation for $E_{A}[D(\tau \mid \mathbf{a})]$ in Eq. (5.16) has more detail than is needed in practice. In particular, when $\lambda_{D}(b-a)$ is "small" and as a result the probabilities $p_{A}\left[\mathcal{A}_{n}(a, b)\right]$ for $n=2,3, \ldots$, are also "small", $E_{A}[D(\tau \mid \mathbf{a})]$ can be adequately represented by

$$
\begin{aligned}
E_{A}[D(\tau \mid \mathbf{a})] & \cong \sum_{n=0}^{1} E_{A}\left\{D\left[\tau \mid \mathbf{a} \in \mathcal{A}_{n}(a, b)\right]\right\} p_{A}\left[\mathcal{A}_{n}(a, b)\right] \\
& \cong \sum_{n=0}^{1} \hat{E}_{A}\left\{D\left[\tau \mid \mathbf{a} \in \mathcal{A}_{n}(a, b)\right]\right\} p_{A}\left[\mathcal{A}_{n}(a, b)\right]
\end{aligned}
$$

as the omitted terms have a negligible effect on the value of $E_{A}[D(\tau \mid \mathbf{a})]$. Further, when $\lambda_{D}(b-a)$ is "small," such omission is consistent with NRC guidance on omitting "very unlikely features, events, or processes" (e.g., see Quotes (NRC6), (NRC9), (NRC13)). However, the representation for $E_{A}[D(\tau \mid \mathbf{a})]$ in Eq. (5.16) is useful because it leads to an alternative representation for $E_{A}[D(\tau \mid \mathbf{a})]$ that forms the basis for an efficient computational structure that can be used in PA for the YM repository.

The indicated alternative representation for $E_{A}[D(\tau \mid \mathbf{a})]$ is now derived. This derivation is predicated on the assumption that the dose $D(\tau \mid \mathbf{a})$ for the element $\mathbf{a}=\left[n, t_{1}, \mathbf{p}_{1}, t_{2}, \mathbf{p}_{2}, \ldots, t_{n}, \mathbf{p}_{n}\right]$ of $\mathcal{A}(a, b)$ can be represented in the following form: 


$$
\begin{aligned}
D(\tau \mid \mathbf{a}) & =D_{N}(\tau \mid \mathbf{a}=[0])+\sum_{i=1}^{n} D_{D}\left(\tau \mid \mathbf{a}_{i}=\left[1, t_{i}, \mathbf{p}_{i}\right]\right) \\
& =D_{N}(\tau)+\sum_{i=1}^{n} D_{D}\left(\tau \mid t_{i}, \mathbf{p}_{i}\right)
\end{aligned}
$$

where

$$
\begin{aligned}
& D_{N}\left(\tau \mid \mathbf{a}_{N}=[0]\right)=\text { dose (mrem/yr) to the RMEI at time } \tau \text { (yr) for nominal (i.e., undisturbed) } \\
& \text { conditions, } \\
& D_{D}\left(\tau \mid \mathbf{a}_{i}=\left[1, t_{i}, \mathbf{p}_{i}\right]\right)=\text { incremental dose (mrem/yr) to the RMEI at time } \tau(\mathrm{yr}) \text { for disturbed conditions } \\
& \text { defined by element } \mathbf{a}_{i}=\left[1, t_{i}, \mathbf{p}_{i}\right] \text { of } \mathcal{A}(a, b) \text { corresponding to one event } \\
& \text { occurring at time } t_{i} \text { with property vector } \mathbf{p}_{i} \text {, }
\end{aligned}
$$

and $D_{N}(\tau)$ and $D_{D}\left(\tau \mid t_{i}, \mathbf{p}_{i}\right)$ are used as more compact representations for $D_{D}\left(\tau \mid \mathbf{a}_{N}=[0]\right)$ and $D_{D}\left(\tau \mid \mathbf{a}_{i}=\left[1, t_{i}, \mathbf{p}_{i}\right]\right)$, respectively. The adjective incremental is used in the definition of $D_{D}\left(\tau \mid \mathbf{a}_{i}=\left[1, t_{i}, \mathbf{p}_{i}\right]\right)=D_{D}\left(\tau \mid t_{i}, \mathbf{p}_{i}\right)$ to emphasize that the indicated dose is in addition to (i.e., incremental to) the dose $D_{N}(\tau \mid \mathbf{a}=[0])=D_{N}(\tau)$ from nominal conditions.

The representation for $D(\tau \mid \mathbf{a})$ in Eq. (5.19) is based on the following three assumptions: (i) the processes that give rise to the nominal dose $D_{N}(\tau)$ are always present and are unaffected by the occurrence of disruptive events; (ii) the disruptive dose $D_{D}\left(\tau \mid t_{i}, \mathbf{p}_{i}\right)$ derives from changed conditions associated with the element $\mathbf{a}_{i}=\left[1, t_{i}, p_{i}\right]$ of $\mathcal{A}(a, b)$ and does not contain any contributions to dose that are already incorporated into $D_{N}(\tau)$; and (iii) there are no synergisms between the individual disruptive occurrences associated with $\mathbf{a}$. Assumptions (i) and (ii) result in $D_{N}(\tau)$ occurring once, and only once, in the representation for $D(\tau \mid \mathbf{a})$. Assumptions (ii) and (iii) result in the sum of the doses from individual disruptive events that are present in the representation for $D(\tau \mid \mathbf{a})$.

The expected value $E_{A}\left[D_{D}(\tau \mid t, \mathbf{p})\right]$ for $D_{D}(\tau \mid t, \mathbf{p})$ is given by

$$
\begin{aligned}
E_{A}\left[D_{D}(\tau \mid t, \mathbf{p})\right] & =\int_{a}^{\tau}\left[\int_{\mathcal{P}} D(\tau \mid t, \mathbf{p}) d_{P}(\mathbf{p}) \mathrm{d} P\right]\left[\frac{1}{\tau-a}\right] \mathrm{d} t \\
& =\frac{1}{\tau-a} \int_{a}^{\tau} \int_{\mathcal{P}} D(\tau \mid t, \mathbf{p}) d_{P}(\mathbf{p}) \mathrm{d} P \mathrm{~d} t,
\end{aligned}
$$

where $1 /(\tau-a)$ is the density function for $t$ as indicated in conjunction with Eq. (4.12), $\mathcal{P}$ is the set of possible values for $\mathbf{p}$ (i.e., $\mathcal{P}$ is the sample space for $\mathbf{p}$ ), and $d_{P}(\mathbf{p})$ is the density function for $\mathbf{p}$ defined on $\mathscr{P}$. In effect, $\mathscr{P}$ and $d_{P}(\mathbf{p})$ define a probability space $\left(\mathcal{P}, \mathbb{P}, p_{P}\right)$ for $\mathbf{p}$ conditional on the occurrence of the disruptive event under consideration (e.g., an igneous event) and also under the assumption that the properties of $\left(\mathscr{P}, \mathbb{P}, p_{P}\right)$ are independent of event time. With respect to the first equality in Eq. (5.22), the inner integral over $\mathscr{P}$ determines expected dose at time $\tau$ given that the event occurred at time $t$ and the outer integral over $[a, \tau]$ incorporates the probability of the event occurring at different times; the second equality results from a simple rearrangement of the first equality. The expected value $E_{A}\left[D_{D}(\tau \mid t, \mathbf{p})\right]$ is a conditional result; specifically, $E_{A}\left[D_{D}(\tau \mid t, \mathbf{p})\right]$ is the expected dose to the RMEI at time $\tau$ conditional on the assumption that exactly one event occurred in the time interval $[a, \tau]$ with properties characterized by the probability space $\left(\mathscr{P}, \mathbb{P}, p_{P}\right)$.

A general and compact representation for $E_{A}[D(\tau \mid \mathbf{a})]$ is now developed. In particular, this representation for $E_{A}[D(\tau \mid \mathbf{a})]$ derives from the following representation for $E_{A}\left\{D\left[\tau \mid \mathbf{a} \in \mathcal{A}_{n}(a, \tau)\right]\right\}$ : 


$$
\begin{aligned}
E_{A}\left\{D\left[\tau \mid \mathbf{a} \in \mathcal{A}_{n}(a, \tau)\right]\right\} & =E_{A}\left\{D_{N}(\tau)+\sum_{i=1}^{n} D_{D}\left\{\tau \mid t_{i}, \mathbf{p}_{i}\right\}\right\} \\
& =D_{N}(\tau)+\sum_{i=1}^{n} E_{A}\left[D_{D}\left(\tau \mid t_{i}, \mathbf{p}_{i}\right)\right] \\
& =D_{N}(\tau)+n\left\{E_{A}\left[D_{D}(\tau \mid t, \mathbf{p})\right]\right\} \\
& =D_{N}(\tau)+\frac{n}{\tau-a} \int_{a}^{\tau} \int_{\mathcal{P}} D_{D}(\tau \mid t, \mathbf{p}) d_{P}(\mathbf{p}) \mathrm{d} P \mathrm{~d} t,
\end{aligned}
$$

where (i) the first equality follows from the assumed form for $D(\tau \mid \mathbf{a})$ in Eq. (5.19) and $\mathcal{A}_{n}(a, \tau)$ is defined analogously to $\mathcal{A}_{n}(a, b)$ in Eq. (5.6) for the interval $[a, \tau]$ rather than the interval $[a, b]$, (ii) the second equality follows from the linearity of expected values, (iii) the third equality follows because the pairs $\left[t_{i}, \mathbf{p}_{i}\right]$ in each dose function $D_{D}\left(\tau \mid t_{i}, \mathbf{p}_{i}\right)$ have the same distribution, and (iv) the fourth and final equality follows from Eq. (5.22). The manipulations in Eq. (5.3) are beneficial in that they convert a complex sum into a relatively simple integral.

The desired representation for $E_{A}[D(\tau \mid \mathbf{a})]$ is now given by

$$
\begin{aligned}
E_{A}[D(\tau \mid \mathbf{a})]= & \sum_{n=0}^{\infty} E_{A}\left\{D\left[\tau \mid \mathbf{a} \in \mathcal{A}_{n}(a, \tau)\right]\right\} p_{A}\left[\mathcal{A}_{n}(a, \tau)\right] \\
= & \sum_{n=0}^{\infty}\left\{D_{N}(\tau)+\frac{n}{\tau-a} \int_{a}^{\tau} \int_{\mathcal{P}} D_{D}(\tau \mid t, \mathbf{p}) d_{P}(\mathbf{p}) \mathrm{d} P \mathrm{~d} t\right\} \\
& \times\left\{\left[\lambda_{D}(\tau-a)\right]^{n} / n !\right\} \exp \left[-\lambda_{D}(\tau-a)\right] \\
= & D_{N}(\tau)+\left\{\int_{a}^{\tau} \int_{\mathcal{P}} D_{D}(\tau \mid t, \mathbf{p}) \lambda_{D} d_{P}(\mathbf{p}) \mathrm{d} P \mathrm{~d} t\right\} \\
& \times\left\{\exp \left[-\lambda_{D}(\tau-a)\right]\right\}\left\{\sum_{n=0}^{\infty}\left[\lambda_{D}(\tau-a)\right]^{n} / n !\right\} \\
= & D_{N}(\tau)+\int_{a}^{\tau} \int_{\mathcal{P}} D_{D}(\tau \mid t, \mathbf{p}) \lambda_{D} d_{P}(\mathbf{p}) \mathrm{d} P \mathrm{~d} t,
\end{aligned}
$$

where (i) the first equality follows from the representation for $E_{A}[D(\tau \mid \mathbf{a})]$ in Eq. (5.14), (ii) the second equality follows from the representations for $E_{A}\left\{D\left[\tau \mid \mathbf{a} \in \mathcal{A}_{n}(a, \tau)\right]\right\}$ and $p_{A}\left[\mathcal{A}_{n}(a, \tau)\right]$ in Eqs. (5.23) and (5.7), respectively, (iii) the third equality results from the identity

$$
1=\sum_{n=0}^{\infty}\left\{\left[\lambda_{D}(\tau-a)\right]^{n} / n !\right\} \exp \left[-\lambda_{D}(\tau-a)\right]
$$

and an algebraic reformulation of the second equality, and (iv) the fourth and final equality again results from the identity in Eq. (5.25).

The steps leading to the representation for $E_{A}[D(\tau \mid \mathbf{a})]$ in Eq. (5.24) are summarized in Table 1, with (i) the first column showing the number $n$ of occurrences that defines scenario class $\mathcal{A}_{n}(a, \tau)$, (ii) the second column summarizing the scenario class probabilities $p_{A}\left[\mathcal{A}_{n}(a, \tau)\right]$, (iii) the third column presenting the form of the dose function $D(\tau \mid \mathbf{a})$ for individual elements of $\mathcal{A}_{n}(a, \tau)$, (iv) the fourth column showing the expected conditional doses $E_{A}\left\{D\left[\tau \mid \mathbf{a} \in \mathcal{A}_{n}(a, \tau)\right]\right\}$, and (v) the fifth and final column showing the product of $E_{A}\left\{D\left[\tau \mid \mathbf{a} \in \mathcal{A}_{n}(a, \tau)\right]\right\}$ and $p_{A}\left[\mathcal{A}_{n}(a, \tau)\right]$, which is the unconditional expected dose associated with scenario class $\mathcal{A}_{n}(a, \tau)$ at time $\tau$. In turn, the expected dose $E_{A}[D(\tau \mid \mathbf{a})]$ is given by the sum of the unconditional expected doses in the final column of the table. 
Table 1. Illustration of Steps Leading to Integral Representation for $E_{A}[D(\tau \mid \mathbf{a})]$ in Eq. (5.24)

\begin{tabular}{|c|c|c|c|c|}
\hline$n^{\mathrm{a}}$ & $p_{A}\left[\mathcal{A}_{n}(a, \tau)\right]^{\mathrm{b}}$ & $D(\tau \mid \mathbf{a})^{\mathrm{c}}$ & $E_{A}\left\{D\left[\tau \mid \mathbf{a} \in \mathcal{A}_{n}(a, \tau)\right]\right\}^{\mathrm{d}}$ & $E_{A}\left\{D\left[\tau \mid \mathbf{a} \in \mathcal{A}_{n}(a, \tau)\right]\right\} \quad p_{A}\left[\mathcal{A}_{n}(a, \tau)\right]^{\mathrm{e}}$ \\
\hline & $\left\{\left[\lambda_{D}(\tau-a)\right]^{0} / 0 !\right\} \exp \left[-\lambda_{D}(\tau-a)\right]$ & $D_{N}(\tau)$ & $D_{N}(\tau)$ & $D_{N}(\tau)\left\{\left[\lambda_{D}(\tau-a)\right]^{0} / 0 !\right\} \exp \left[-\lambda_{D}(\tau-a)\right.$ \\
\hline & $\left\{\left[\lambda_{D}(a-\tau)\right]^{1} / 1 !\right\} \exp \left[-\lambda_{D}(\tau-a)\right]$ & $D_{N}(\tau)+\sum_{i=1}^{1} D_{D}\left(\tau \mid t_{i}, \mathbf{p}_{i}\right)$ & $D_{N}(\tau)+\frac{1}{\tau-a} \int_{a}^{\tau} \int_{\mathcal{P}} D_{D}(\tau \mid t, \mathbf{p}) d_{P}(\mathbf{p}) \mathrm{d} P \mathrm{~d} t$ & $\begin{array}{l}\left\{D_{N}(\tau)+\frac{1}{\tau-a} \int_{a}^{\tau} \int_{\mathcal{P}} D_{D}(\tau \mid t, \mathbf{p}) d_{P}(\mathbf{p}) \mathrm{d} P \mathrm{~d} t\right. \\
\quad \times\left\{\left[\lambda_{D}(\tau-a)\right] / 1 !\right\} \exp \left[-\lambda_{D}(\tau-a)\right]\end{array}$ \\
\hline & $\left\{\left[\lambda_{D}(a-\tau)\right]^{2} / 2 !\right\} \exp \left[-\lambda_{D}(\tau-a)\right]$ & $D_{N}(\tau)+\sum_{i=1}^{2} D_{D}\left(\tau \mid t_{i}, \mathbf{p}_{i}\right)$ & $D_{N}(\tau)+\frac{2}{\tau-a} \int_{a}^{\tau} \int_{\mathscr{P}} D_{D}(\tau \mid t, \mathbf{p}) d_{P}(\mathbf{p}) \mathrm{d} P \mathrm{~d} t$ & $\begin{array}{l}\left\{D_{N}(\tau)+\frac{2}{\tau-a} \int_{a}^{\tau} \int_{\mathscr{P}} D_{D}(\tau \mid t, \mathbf{p}) d_{P}(\mathbf{p}) \mathrm{d} P \mathrm{~d} t\right. \\
\quad \times\left\{\left[\lambda_{D}(\tau-a)\right]^{2} / 2 !\right\} \exp \left[-\lambda_{D}(\tau-a)\right]\end{array}$ \\
\hline 3 & $\left\{\left[\lambda_{D}(a-\tau)\right]^{3} / 3 !\right\} \exp \left[-\lambda_{D}(\tau-a)\right]$ & $D_{N}(\tau)+\sum_{i=1}^{3} D_{D}\left(\tau \mid t_{i}, \mathbf{p}_{i}\right)$ & $D_{N}(\tau)+\frac{3}{\tau-a} \int_{a}^{\tau} \int_{\mathscr{P}} D_{D}(\tau \mid t, \mathbf{p}) d_{P}(\mathbf{p}) \mathrm{d} P \mathrm{~d} t$ & $\begin{array}{l}\left\{D_{N}(\tau)+\frac{3}{\tau-a} \int_{a}^{\tau} \int_{\mathscr{P}} D_{D}(\tau \mid t, \mathbf{p}) d_{P}(\mathbf{p}) \mathrm{d} P \mathrm{~d} t\right. \\
\quad \times\left\{\left[\lambda_{D}(\tau-a)\right]^{3} / 3 !\right\} \exp \left[-\lambda_{D}(\tau-a)\right]\end{array}$ \\
\hline$\vdots$ & $\vdots$ & $\vdots$ & $\vdots$ & $\vdots$ \\
\hline
\end{tabular}

\footnotetext{
a Number $n$ of occurrences that defines scenario class $\mathcal{A}_{n}(a, \tau)$; see Eq. (5.6).

b Probability $p_{A}\left[\mathcal{A}_{n}(a, \tau)\right]$ of scenario class $\mathcal{A}_{n}(a, \tau)$; see Eq. (5.7).

c Form of dose function $D(\tau \mid \mathbf{a})$ for individual elements of scenario class $\mathcal{A}_{n}(a, \tau)$; see Eq. (5.19).

d Conditional expected dose $E_{A}\left\{D\left[\tau \mid \mathbf{a} \in \mathcal{A}_{n}(a, \tau)\right]\right\}$ for scenario class $\mathcal{A}_{n}(a$, $\tau)$; see Eq. (5.23).

e Unconditional expected dose $E_{A}\left\{D\left[\tau \mid \mathbf{a} \in \mathcal{A}_{n}(a, \tau)\right]\right\} \quad p_{A}\left[\mathcal{A}_{n}(a, \tau)\right]$ for scenario class $\mathcal{A}_{n}(a, \tau)$.

f Expected dose $E_{A}[D(\tau \mid \mathbf{a})]$; see Eq. (5.24).
} 
As indicated in Eq. (5.18), the representation for $E_{A}[D(\tau \mid \mathbf{a})]$ can be simplified by only considering the scenario classes $\mathcal{A}_{0}(a, b)$ and $\mathcal{A}_{1}(a, b)$. However, as can be seen from the derivation leading to Eq. (5.24), representations of this type are not always advantageous. In particular, retention of the scenario classes $\mathcal{A}_{n}(a, b), n=2,3, \ldots$, provides the basis for a clear mathematical path to the integral representation for $E_{A}[D(\tau \mid \mathbf{a})]$ in Eq. (5.24) that can be used as the basis for the calculation of approximations $\hat{E}_{A}[D(\tau \mid \mathbf{a})]$ to $E_{A}[D(\tau \mid \mathbf{a})]$ in PAs for the YM repository. Thus, even though the scenario classes $\mathcal{A}_{n}(a, b), n=2,3, \ldots$, may not contribute significantly to $E_{A}[D(\tau \mid \mathbf{a})]$, their retention helps in developing an unambiguous representation for the structure of a PA for the YM repository. Further, use of the representation for $E_{A}[D(\tau \mid \mathbf{a})]$ in Eq. (5.24) makes it possible to state correctly that multiple disruptive events are included in the analysis.

Given the just completed derivation for $E_{A}[D(\tau \mid \mathbf{a})]$ in Eq. (5.24), this is a convenient point to consider the representation of different types of disruptions in PAs for the YM repository. As previously indicated in Sect. 3, PAs for the YM repository need to consider two types of disruptions: (i) igneous occurrences that follow a Poisson process with a rate constant $\lambda_{I}$, and (ii) seismic occurrences that follow a Poisson process with a rate constant $\lambda_{S}$. Further, the properties of an igneous occurrence are characterized by a vector $\mathbf{p}_{I}$ from a set $I$ with a density function $d_{I}\left(\mathbf{p}_{I}\right)$, and similarly, the properties of a seismic occurrence are characterized by a vector $\mathbf{p}_{S}$ from a set $S$ with a density function $d_{S}\left(\mathbf{p}_{S}\right)$.

With both igneous and seismic occurrences under consideration, possible futures that could occur over the time interval $[a, b]$ can be represented by vectors of the form

$$
\mathbf{a}=\left[m, n, t_{I 1}, \mathbf{p}_{I 1}, \ldots, t_{I m}, \mathbf{p}_{I m}, t_{S 1}, \mathbf{p}_{S 1}, \ldots, t_{S n}, \mathbf{p}_{S n}\right]
$$

where (i) $m$ and $n$ are numbers of igneous and seismic occurrences, respectively, (ii) $a \leq t_{I 1} \leq \ldots t_{I m} \leq b$ and $\mathbf{p}_{I 1}, \ldots$, $\mathbf{p}_{I m}$ are the times and property vectors for the individual igneous occurrences, and (iii) $a \leq t_{S 1} \leq \ldots t_{S n} \leq b$ and $\mathbf{p}_{S 1}$, $\ldots, \mathbf{p}_{S n}$ are the times and property vectors for the individual seismic occurrences. Then,

$$
\mathcal{A}(a, b)=\{\mathbf{a}: \mathbf{a}=[m, n, \ldots] \text { is a vector of the form in Eq. (5.26) for } m=0,1,2, \ldots, n=0,1,2, \ldots\}
$$

represents the set of all sequences of occurrences (i.e., futures) over the time interval $[a, b]$, with

$$
\mathbf{a}_{N}=\mathbf{a}_{N}(a, b)=[0,0]
$$

understood to represent the future with no disruptive occurrences over the time interval $[a, b]$ (i.e., the nominal future). Further, individual scenario classes can be defined by

$$
\mathcal{A}_{m n}(a, b)=\{\mathbf{a}: \mathbf{a}=[m, n, \ldots] \text { is a vector of the form in Eq. (5.26) }\}
$$

for $m=0,1,2, \ldots$ and $n=0,1,2, \ldots$

Similarly to the assumptions that underlie the derivation leading to the representation for $E_{A}[D(\tau \mid \mathbf{a})]$ in Eq. (5.24), it is assumed that $D(\tau \mid \mathbf{a})$ can be represented in the following form for elements $\mathbf{a}$ of the set $\mathcal{A}(a, b)$ in Eq. (5.27):

$$
\begin{aligned}
D(\tau \mid \mathbf{a}) & =D\left(\tau \mid \mathbf{a}_{N}=[0,0]\right)+\sum_{i=1}^{m} D_{I}\left(\tau \mid \mathbf{a}_{i}=\left[1,0, t_{I i}, \mathbf{p}_{I i}\right]\right)+\sum_{i=1}^{n} D_{S}\left(\tau \mid \mathbf{a}_{i}=\left[0,1, t_{S i}, \mathbf{p}_{S i}\right]\right) \\
& =D_{N}(\tau)+\sum_{i=1}^{m} D_{I}\left(\tau \mid t_{I i}, \mathbf{p}_{I i}\right)+\sum_{i=1}^{n} D_{S}\left(\tau \mid t_{S i}, \mathbf{p}_{S i}\right)
\end{aligned}
$$

where 


$$
\begin{aligned}
& D_{N}\left(\tau \mid \mathbf{a}_{N}=[0,0]\right)=\text { dose (mrem } / \mathrm{yr}) \text { to the RMEI at time } \tau(\mathrm{yr}) \text { for nominal (i.e., undisturbed) } \\
& \text { conditions, }
\end{aligned}
$$

$$
\begin{aligned}
& D_{I}\left(\tau \mid \mathbf{a}_{i}=\left[1,0, t_{I i}, p_{I i}\right]\right)=\text { incremental dose (mrem/yr) to the RMEI at time } \tau \text { (yr) for disturbed (i.e., igneous) } \\
& \text { conditions defined by element } \mathbf{a}_{i}=\left[1,0, t_{I i}, \mathbf{p}_{I i}\right] \text { of } \mathcal{A}(a, b) \text {, }
\end{aligned}
$$

and $D_{N}(\tau), D_{I}\left(\tau \mid t_{I i}, \mathbf{p}_{I i}\right)$ and $D_{S}\left(\tau \mid t_{S i}, \mathbf{p}_{S i}\right)$ are used as compact representations for $D_{N}\left(\tau \mid \mathbf{a}_{N}=[0,0]\right), D_{I}\left(\tau \mid \mathbf{a}_{i}=[1,0\right.$, $\left.\left.t_{I i}, \mathbf{p}_{I i}\right]\right)$ and $D_{S}\left(\tau \mid \mathbf{a}_{i}=\left[1,0, t_{S i}, \mathbf{p}_{S i}\right]\right)$, respectively.

Given the representation for $D(\tau \mid \mathbf{a})$ in Eq. (5.30), $E_{A}[D(\tau \mid \mathbf{a})]$ can be determined for elements of the set $\mathcal{A}(a, b)$ defined in Eq. (5.27) in the same manner as $E_{A}[D(\tau \mid \mathbf{a})]$ was determined in Eq. (5.24) for elements of the set $\mathcal{A}(a, b)$ defined in Eq. (5.2). Specifically,

$$
\begin{aligned}
E_{A}[D(\tau \mid \mathbf{a})] & =\sum_{m=0}^{\infty} \sum_{n=0}^{\infty} E_{A}\left\{D\left(\tau \mid \mathbf{a} \in \mathcal{A}_{m n}(a, \tau)\right]\right\} p_{A}\left[\mathcal{A}_{m n}(a, \tau)\right] \\
= & \sum_{m=0}^{\infty} \sum_{n=0}^{\infty}\left\{D_{N}(\tau)+\frac{m}{\tau-a} \int_{a}^{\tau} \int_{I} D_{I}\left(\tau \mid t, \mathbf{p}_{I}\right) d_{I}\left(\mathbf{p}_{I}\right) \mathrm{d} I \mathrm{~d} t+\frac{n}{\tau-a} \int_{a}^{\tau} \int_{S} D_{S}\left(\tau \mid t, \mathbf{p}_{S}\right) d_{S}\left(\mathbf{p}_{S}\right) \mathrm{d} S \mathrm{~d} t\right\} \\
& \times\left\{\left[\lambda_{I}(\tau-a)\right]^{m} / m !\right\}\left\{\left[\lambda_{S}(\tau-a)\right]^{n} / n !\right\} \exp \left[-\left(\lambda_{I}+\lambda_{S}\right)(\tau-a)\right] \\
= & D_{N}(\tau)+\left(\int_{a}^{\tau} \int_{I} D_{I}\left(\tau \mid t, \mathbf{p}_{I}\right) \lambda_{I} d_{I}\left(\mathbf{p}_{I}\right) \mathrm{d} I \mathrm{~d} t+\int_{a}^{\tau} \int_{S} D_{S}\left(\tau \mid t, \mathbf{p}_{S}\right) \lambda_{S} d_{S}\left(\mathbf{p}_{S}\right) \mathrm{d} S \mathrm{~d} t\right) \\
& \times\left(\sum_{m=0}^{\infty} \sum_{n=0}^{\infty}\left\{\left[\lambda_{I}(\tau-a)\right]^{m} / m !\right\}\left\{\left[\lambda_{S}(\tau-a)\right]^{n} / n !\right\}\right) \exp \left[-\left(\lambda_{I}+\lambda_{S}\right)(\tau-a)\right] \\
= & D_{N}(\tau)+\int_{a}^{\tau} \int_{I} D_{I}\left(\tau \mid t, \mathbf{p}_{I}\right) \lambda_{I} d_{I}\left(\mathbf{p}_{I}\right) \mathrm{d} I \mathrm{~d} t+\int_{a}^{\tau} \int_{S} D_{S}\left(\tau \mid t, \mathbf{p}_{S}\right) \lambda_{S} d_{S}\left(\mathbf{p}_{S}\right) \mathrm{d} S \mathrm{~d} t .
\end{aligned}
$$

The preceding decomposition of expected dose $E_{A}[D(\tau \mid \mathbf{a})]$ into dose from nominal conditions and incremental doses from igneous and seismic occurrences and the conversion of infinite sums into integrals provides an overarching computational structure on which a PA for the YM repository can be based. In particular, it is possible to estimate the three dose components in Eq. (5.34) separately and then add these estimates to obtain an estimate for $E_{A}[D(\tau \mid \mathbf{a})]$

Probability is neither lost nor gained in the derivation leading to the representation for $E_{A}[D(\tau \mid \mathbf{a})]$ in Eq. (5.34). As a result, this representation for $E_{A}[D(\tau \mid \mathbf{a})]$ is consistent with the guidance in Quote (YMRP3) that "The probabilities of occurrence of all scenario classes, included in calculating the annual dose curve, sum to one." However, the assumption is made that there are no synergisms between the effects associated with multiple disruptive occurrences. As long as the occurrence rates $\lambda_{I}$ and $\lambda_{S}$ are small relative to the time interval under consideration, the likelihood of multiple occurrences is also small and the indicated assumption of no synergisms is of little consequence. 
This page intentionally left blank. 


\section{Expected Dose with Epistemic Uncertainty}

Many quantities used in PA for the YM repository are uncertain in an epistemic sense. Some of these uncertain quantities are involved in the calculation of the doses $D_{N}(\tau), D_{I}\left(\tau \mid t, \mathbf{p}_{I}\right)$ and $D_{S}\left(\tau \mid t, \mathbf{p}_{S}\right)$ appearing in Eq. (5.34); others of these quantities relate to uncertainty in the PA inputs used to characterize aleatory uncertainty such as $\lambda_{I}$, $\lambda_{S}, d_{I}\left(\mathbf{p}_{I}\right)$ and $d_{S}\left(\mathbf{p}_{S}\right)$. The importance of an appropriate treatment of epistemic uncertainty is emphasized by the NRC in a number of statements (e.g., see Quotes (NRC3), (NRC10) - (NRC15), (YMRP10)). Further, an appropriate treatment of epistemic uncertainty is basic to answering Question (Q4).

For notational convenience, uncertain quantities involved in the characterization of aleatory uncertainty will be represented by a vector

$$
\mathbf{e}_{A}=\left[e_{A 1}, e_{A 2}, \ldots, e_{A, n A}\right],
$$

and uncertain quantities involved in the evaluation of $D_{N}(\tau), D_{I}\left(\tau \mid t, \mathbf{p}_{I}\right)$ and $D_{S}\left(\tau \mid t, \mathbf{p}_{S}\right)$ will be represented by a vector

$$
\mathbf{e}_{D}=\left[e_{D 1}, e_{D 2}, \ldots, e_{D, n D}\right]
$$

Then, the vector

$$
\mathbf{e}=\left[\mathbf{e}_{A}, \mathbf{e}_{D}\right]=\left[e_{1}, e_{2}, \ldots, e_{n E}\right]
$$

contains the $n E=n A+n D$ epistemically uncertain variables considered in a PA for the YM repository .

Different values for the elements of the vector $\mathbf{e}=\left[\mathbf{e}_{A}, \mathbf{e}_{D}\right]$ defined in conjunction with Eqs. (6.1) - (6.3) lead to different values for the expected dose $E_{A}[D(\tau \mid \mathbf{a})]$. Thus, $E_{A}[D(\tau \mid \mathbf{a})]$ is actually a function of $\mathbf{e}$; or put another way, the value for $E_{A}[D(\tau \mid \mathbf{a})]$ is conditional on $\mathbf{e}$. When this conditionality is explicitly recognized, the representation for $E_{A}[D(\tau \mid \mathbf{a})]$ in Eq. (5.24) becomes

$$
E_{A}\left[D\left(\tau \mid \mathbf{a}, \mathbf{e}_{D}\right) \mid \mathbf{e}_{A}\right]=D_{N}\left(\tau \mid \mathbf{e}_{D}\right)+\int_{a}^{\tau} \int_{\mathcal{P}} D_{D}\left(\tau \mid t, \mathbf{p}, \mathbf{e}_{D}\right) \lambda_{D} d_{P}\left(\mathbf{p} \mid \mathbf{e}_{A}\right) \mathrm{d} P \mathrm{~d} t,
$$

and the more general representation for $E_{A}[D(\tau \mid \mathbf{a})]$ in Eq. (5.34) becomes

$$
\begin{aligned}
E_{A}\left[D\left(\tau \mid \mathbf{a}, \mathbf{e}_{D}\right) \mid \mathbf{e}_{A}\right]= & D_{N}\left(\tau \mid \mathbf{e}_{D}\right)+\int_{a}^{\tau} \int_{I} D_{I}\left(\tau \mid t, \mathbf{p}_{I}, \mathbf{e}_{D}\right) \lambda_{I} d_{I}\left(\mathbf{p}_{I} \mid \mathbf{e}_{A}\right) \mathrm{d} I \mathrm{~d} t \\
& +\int_{a}^{\tau} \int_{S} D_{S}\left(\tau \mid t, \mathbf{p}_{S}, \mathbf{e}_{D}\right) \lambda_{S} d_{S}\left(\mathbf{p}_{S} \mid \mathbf{e}_{A}\right) \mathrm{d} S \mathrm{~d} t,
\end{aligned}
$$

with the notation indicating that the dose functions $D_{N}\left(\tau \mid \mathbf{e}_{D}\right), D_{D}\left(\tau \mid t, \mathbf{p}, \mathbf{e}_{D}\right), D_{I}\left(\tau \mid t, \mathbf{p}_{I}, \mathbf{e}_{D}\right)$ and $D_{S}\left(\tau \mid t, \mathbf{p}_{S}, \mathbf{e}_{D}\right)$ have values that depend on $\mathbf{e}_{D}$ and that the density functions $d_{D}\left(\mathbf{p} \mid \mathbf{e}_{A}\right), d_{I}\left(\mathbf{p}_{I} \mid \mathbf{e}_{A}\right)$ and $d_{S}\left(\mathbf{p}_{S} \mid \mathbf{e}_{A}\right)$ have values that depend on $\mathbf{e}_{A}$. Further, it is tacitly assumed that the values for $\lambda_{D}, \lambda_{I}$ and $\lambda_{S}$ could be elements of $\mathbf{e}_{A}$.

When needed, the notations

$$
\begin{aligned}
& E_{A}\left[D_{D}\left(\tau \mid \mathbf{a}, \mathbf{e}_{D}\right) \mid \mathbf{e}_{A}\right]=\int_{a}^{\tau} \int_{\mathcal{P}} D_{D}\left(\tau \mid t, \mathbf{p}, \mathbf{e}_{D}\right) \lambda_{D} d_{P}\left(\mathbf{p} \mid \mathbf{e}_{A}\right) \mathrm{d} P \mathrm{~d} t \\
& E_{A}\left[D_{I}\left(\tau \mid \mathbf{a}, \mathbf{e}_{D}\right) \mid \mathbf{e}_{A}\right]=\int_{a}^{\tau} \int_{I} D_{I}\left(\tau \mid t, \mathbf{p}_{I}, \mathbf{e}_{D}\right) \lambda_{I} d_{I}\left(\mathbf{p}_{I} \mid \mathbf{e}_{A}\right) \mathrm{d} I \mathrm{~d} t
\end{aligned}
$$

and 


$$
E_{A}\left[D_{S}\left(\tau \mid \mathbf{a}, \mathbf{e}_{D}\right) \mid \mathbf{e}_{A}\right]=\int_{a}^{\tau} \int_{S} D_{S}\left(\tau \mid t, \mathbf{p}_{S}, \mathbf{e}_{D}\right) \lambda_{S} d_{S}\left(\mathbf{p}_{S} \mid \mathbf{e}_{A}\right) \mathrm{d} S \mathrm{~d} t
$$

are used to represent incremental expected dose from individual disruptive scenario classes conditional on a specific vector $\mathbf{e}=\left[\mathbf{e}_{A}, \mathbf{e}_{D}\right]$ of values for epistemically uncertain analysis inputs.

The NRC indicates that PAs for the YM repository should use probability to provide a mathematical characterization of the epistemic uncertainty associated with the elements of $\mathbf{e}=\left[\mathbf{e}_{A}, \mathbf{e}_{D}\right]$ (e.g., see Quotes (NRC3), (NRC10), (NRC11)). In particular, the epistemic uncertainty associated with each element $e_{i}, i=1,2, \ldots, n A+n D$, of $\mathbf{e}$ is characterized by a probability distribution $D_{i}$. Thus, the epistemic uncertainty associated with a PA for the YM facility is characterized by a sequence

$$
D_{i}, i=1,2, \ldots, n A+n D=n E,
$$

of probability distributions. For notational convenience, the preceding distributions can be represented by a corresponding density function

$$
d_{E}(\mathbf{e})=d_{A}\left(\mathbf{e}_{A}\right) d_{D}\left(\mathbf{e}_{D}\right),
$$

where $d_{A}\left(\mathbf{e}_{A}\right)$ and $d_{D}\left(\mathbf{e}_{D}\right)$ are density functions associated with $\mathbf{e}_{A}$ and $\mathbf{e}_{D}$, respectively. Further, the set of all possible values for $\mathbf{e}$ consistent with the distributions in Eq. (6.9) can be represented by

$$
\mathcal{E}=\mathcal{E} \mathcal{A} \times \mathcal{E} \mathcal{D},
$$

where $\mathcal{E A}$ and $\mathcal{E} D$ are the sets of all possible values for $\mathbf{e}_{A}$ and $\mathbf{e}_{D}$, respectively. Thus, $\mathcal{E}$ is the sample space for epistemic uncertainty containing the possible values for $\mathbf{e}$, and $\mathcal{E} \mathcal{A}$ and $\mathcal{E D}$ are the corresponding sample spaces for $\mathbf{e}_{A}$ and $\mathbf{e}_{D}$. In effect, a probability space $\left(\mathcal{E}, \mathbb{E}, p_{E}\right)$ for epistemic uncertainty is being defined as indicated in Sect. 3.

The presence and associated probabilistic characterization of epistemic uncertainty means that expected dose to the RMEI derives from both aleatory and epistemic uncertainty. When the epistemic uncertainty associated with the expected dose $E_{A}\left[D\left(\tau \mid \mathbf{a}, \mathbf{e}_{D}\right) \mid \mathbf{e}_{A}\right]$ is taken into account, a new expected dose $E_{E}\left\{E_{A}\left[D\left(\tau \mid \mathbf{a}, \mathbf{e}_{D}\right) \mid \mathbf{e}_{A}\right]\right\}$ is obtained that incorporates the effects of both aleatory and epistemic uncertainty. Specifically,

$$
E_{E}\left\{E_{A}\left[D\left(\tau \mid \mathbf{a}, \mathbf{e}_{D}\right) \mid \mathbf{e}_{A}\right]\right\}=\int_{E} E_{A}\left[D\left(\tau \mid \mathbf{a}, \mathbf{e}_{D}\right) \mid \mathbf{e}_{A}\right] d_{E}(\mathbf{e}) \mathrm{d} E
$$

In turn,

$$
\begin{gathered}
E_{E}\left\{E_{A}\left[D\left(\tau \mid \mathbf{a}, \mathbf{e}_{D}\right) \mid \mathbf{e}_{A}\right]\right\}=\int_{\mathcal{E}}\left\{D_{N}\left(\tau \mid \mathbf{e}_{D}\right)+\int_{a}^{\tau} \int_{\mathcal{P}} D_{D}\left(\tau \mid t, \mathbf{p}, \mathbf{e}_{D}\right) \lambda_{D} d_{P}\left(\mathbf{p} \mid \mathbf{e}_{A}\right) \mathrm{d} P \mathrm{~d} t\right\} d_{E}(\mathbf{e}) \mathrm{d} E \\
=\int_{\mathcal{E} D} D_{N}\left(\tau \mid \mathbf{e}_{D}\right) d_{D}\left(\mathbf{e}_{D}\right) \mathrm{d} E D+\int_{\mathcal{E}} \int_{a}^{\tau} \int_{\mathcal{P}} D_{D}\left(\tau \mid t, \mathbf{p}, \mathbf{e}_{D}\right) \lambda_{D} d_{P}\left(\mathbf{p} \mid \mathbf{e}_{A}\right) d_{E}(\mathbf{e}) \mathrm{d} P \mathrm{~d} t \mathrm{~d} E
\end{gathered}
$$

for the representation for $E_{A}\left[D\left(\tau \mid \mathbf{a}, \mathbf{e}_{D}\right) \mid \mathbf{e}_{A}\right]$ in Eq. (6.4), and

$$
\begin{aligned}
E_{E}\left\{E_{A}\left[D\left(\tau \mid \mathbf{a}, \mathbf{e}_{D}\right) \mid \mathbf{e}_{A}\right]\right\}=\int_{\mathcal{E}}\left\{D_{N}\left(\tau \mid \mathbf{e}_{D}\right)+\int_{a}^{\tau} \int_{I} D_{I}\left(\tau \mid t, \mathbf{p}_{I}, \mathbf{e}_{D}\right) \lambda_{I} d_{I}\left(\mathbf{p}_{I} \mid \mathbf{e}_{A}\right) \mathrm{d} P \mathrm{~d} t\right. \\
\left.\quad+\int_{a}^{\tau} \int_{S} D_{S}\left(\tau \mid t, \mathbf{p}_{S}, \mathbf{e}_{D}\right) \lambda_{S} d_{S}\left(\mathbf{p}_{S} \mid \mathbf{e}_{A}\right) \mathrm{d} S \mathrm{~d} t\right\} d_{E}(\mathbf{e}) \mathrm{d} E \\
=\int_{\mathcal{E} D} D_{N}\left(\tau \mid \mathbf{e}_{D}\right) d_{D}\left(\mathbf{e}_{D}\right) \mathrm{d} E D+\int_{\mathcal{E}} \int_{a}^{\tau} \int_{I} D_{I}\left(\tau \mid t, \mathbf{p}_{I}, \mathbf{e}_{D}\right) \lambda_{I} d_{I}\left(\mathbf{p}_{I} \mid \mathbf{e}_{A}\right) d_{E}(\mathbf{e}) \mathrm{d} I \mathrm{~d} t \mathrm{~d} E \\
\quad+\int_{E} \int_{a}^{\tau} \int_{S} D_{S}\left(\tau \mid t, \mathbf{p}_{S}, \mathbf{e}_{D}\right) \lambda_{S} d_{S}\left(\mathbf{p}_{S} \mid \mathbf{e}_{A}\right) d_{E}(\mathbf{e}) \mathrm{d} S \mathrm{~d} t \mathrm{~d} E
\end{aligned}
$$


for the representation for $E_{A}\left[D\left(\tau \mid \mathbf{a}, \mathbf{e}_{D}\right) \mid \mathbf{e}_{A}\right]$ in Eq. (6.5).

When needed, the notation

$$
E_{E}\left[D_{N}\left(\tau \mid \mathbf{e}_{D}\right)\right]=\int_{E \mathcal{D}} D_{N}\left(\tau \mid \mathbf{e}_{D}\right) d_{D}\left(\mathbf{e}_{D}\right) \mathrm{d} E D
$$

is used to represent expected dose for the nominal scenario class deriving from epistemic uncertainty. Further,

$$
\begin{aligned}
E_{E}\left\{E_{A}\left[D_{D}\left(\tau \mid \mathbf{a}, \mathbf{e}_{D}\right) \mid \mathbf{e}_{A}\right]\right\} & =\int_{\mathcal{E}} E_{A}\left[D_{D}(\tau \mid \mathbf{a}) \mid \mathbf{e}\right] d_{E}(\mathbf{e}) \mathrm{d} E \\
& =\int_{\mathcal{E}} \int_{a}^{\tau} \int_{\mathcal{P}} D_{D}\left(\tau \mid t, \mathbf{p}, \mathbf{e}_{D}\right) \lambda_{D} d_{P}\left(p \mid \mathbf{e}_{A}\right) d_{E}(\mathbf{e}) \mathrm{d} P \mathrm{~d} t \mathrm{~d} E
\end{aligned}
$$

and the similarly defined notations $E_{E}\left\{E_{A}\left[D_{I}\left(\tau \mid \mathbf{a}, \mathbf{e}_{D}\right) \mid \mathbf{e}_{A}\right]\right\}$ and $E_{E}\left\{E_{A}\left[D_{S}\left(\tau \mid \mathbf{a}, \mathbf{e}_{D}\right) \mid \mathbf{e}_{A}\right]\right\}$ are used to represent incremental expected doses from individual disruptive scenario classes that incorporate both aleatory and epistemic uncertainty. 
This page intentionally left blank. 


\section{Calculation of Expected Dose and Display of Epistemic Uncertainty for $D_{N}\left(\tau \mid \mathbf{e}_{D}\right)$}

In concept, the expected value $E_{E}\left[D_{N}\left(\tau \mid \mathbf{e}_{D}\right)\right]$ for $D_{N}\left(\tau \mid \mathbf{e}_{D}\right)$ defined in Eq. (6.15) could be estimated by some type of traditional quadrature-based numerical procedure. As a simple example, $E_{E}\left[D_{N}\left(\tau \mid \mathbf{e}_{D}\right)\right]$ could be estimated by

$$
\hat{E}_{E}\left[D_{N}\left(\tau \mid \mathbf{e}_{D}\right)\right]=\sum_{i=1}^{n V} D_{N}\left(\tau \mid \mathbf{e}_{D i}\right) d_{D}\left(\mathbf{e}_{D i}\right) V\left(\mathcal{E} \mathcal{D}_{i}\right)
$$

where (i) $\mathcal{E} \mathscr{D}_{i}, i=1,2, \ldots, n V$, is a sequence of rectangular volumes that potentially intersect only along their boundaries and satisfy the equality $E \mathcal{E D}=\bigcup_{i=1}^{n V} E \mathcal{D}_{i}$, (ii) $\mathbf{e}_{D i}$ is a point from $\mathcal{E} \mathscr{D}_{i}$, and (iii) $V\left(E \mathcal{D}_{i}\right)$ is the volume of $E \mathcal{D}_{i}$. Then, if $D_{N}\left(\tau \mid \mathbf{e}_{D}\right)$ and $d_{D}\left(\mathbf{e}_{D}\right)$ are reasonably well-behaved, the summation in Eq. (7.1) converges to $E_{E}\left[D_{N}\left(\tau \mid \mathbf{e}_{D}\right)\right]$ as $V\left(E \mathcal{D}_{i}\right)$ goes to zero. Approximations that are more complex and more rapidly convergent are also possible. ${ }^{118}$ Unfortunately, a closed form evaluation for $E_{E}\left[D_{N}\left(\tau \mid \mathbf{e}_{D}\right)\right]$ (i.e., by taking antiderivatives and use of the fundamental theorem of calculus) is unlikely to be possible in any real analysis.

The cumulative distribution function (CDF) that characterizes the epistemic uncertainty in the possible values for $D_{N}\left(\tau \mid \mathbf{e}_{D}\right)$ is defined by

$$
\begin{aligned}
p_{E}\left[D_{N}\left(\tau \mid \mathbf{e}_{D}\right) \leq D\right] & =\text { epistemic probability of a dose } D_{N}\left(\tau \mid \mathbf{e}_{D}\right) \text { less than } D \text { at time } \tau \\
& =\int_{\mathcal{E D}} \underline{\delta}_{D}\left[D_{N}\left(\tau \mid \mathbf{e}_{D}\right)\right] d_{D}\left(\mathbf{e}_{D}\right) \mathrm{d} E D
\end{aligned}
$$

where

$$
\underline{\delta}_{D}\left[D_{N}\left(\tau \mid \mathbf{e}_{D}\right)\right]= \begin{cases}1 & \text { if } D_{N}\left(\tau \mid \mathbf{e}_{D}\right) \leq D \\ 0 & \text { if } D_{N}\left(\tau \mid \mathbf{e}_{D}\right)>D\end{cases}
$$

as previously indicated in conjunction with Eq. (3.4). Similarly to the expected value $E_{E}\left[D_{N}\left(\tau \mid \mathbf{e}_{D}\right)\right]$, the probability $p_{E}\left[D_{N}\left(\tau \mid \mathbf{e}_{D}\right) \leq D\right]$ can be approximated by

$$
\hat{p}_{E}\left[D_{N}\left(\tau \mid \mathbf{e}_{D}\right) \leq D\right]=\sum_{i=1}^{n S} \underline{\delta}_{D}\left[D_{N}\left(\tau \mid \mathbf{e}_{D i}\right)\right] d_{D}\left(\mathbf{e}_{D i}\right) V\left(\mathcal{E} \mathcal{D}_{i}\right)
$$

where the individual terms are defined the same as in Eq. (7.1).

In practice, the complexity of $D_{N}\left(\tau \mid \mathbf{e}_{D}\right)$ and the high dimensionality of $\mathbf{e}_{D}$ make quadrature-based approaches unlikely candidates for the evaluation of the integrals that define $E_{E}\left[D_{N}\left(\tau \mid \mathbf{e}_{D}\right)\right]$ and $p_{E}\left[D_{N}\left(\tau \mid \mathbf{e}_{D}\right) \leq D\right]$. Rather, the usual approach is to use a sampling-based procedure. In particular, a random sample or a Latin hypercube sample (LHS) $)^{119,} 120$

$$
\mathbf{e}_{D i}, i=1,2, \ldots, n S,
$$

is generated from $E \mathscr{D}$ in consistency with the distributions associated with the density functions for the individual elements of $\mathbf{e}_{D}$. Then, $E_{E}\left[D_{N}\left(\tau \mid \mathbf{e}_{D}\right)\right]$ and $p_{E}\left[D_{N}\left(\tau \mid \mathbf{e}_{D}\right) \leq D\right]$ are approximated by

$$
\hat{E}_{E}\left[D_{N}\left(\tau \mid \mathbf{e}_{D}\right)\right]=\sum_{i=1}^{n S} D_{N}\left(\tau \mid \mathbf{e}_{D i}\right) / n S
$$

and 


$$
\hat{p}_{E}\left[D_{N}\left(\tau \mid \mathbf{e}_{D}\right) \leq D\right]=\sum_{i=1}^{n S} \underline{\delta}_{D}\left[D_{N}\left(\tau \mid \mathbf{e}_{D i}\right)\right] / n S
$$

respectively. In most real analyses, this approach is simpler and computationally more efficient than a quadraturebased approach to the evaluation of the integrals that define $E_{E}\left[D_{N}\left(\tau \mid \mathbf{e}_{D}\right)\right]$ and $p_{E}\left[D_{N}\left(\tau \mid \mathbf{e}_{D}\right) \leq D\right]$. Further, it also provides a mapping

$$
\left[\mathbf{e}_{D i}, D_{N}\left(\tau \mid \mathbf{e}_{D i}\right)\right], i=1,2, \ldots, n S,
$$

between analysis inputs and analysis results that constitutes the starting point for a variety of sampling-based sensitivity analysis procedures. ${ }^{121-129}$

The use of sampling-based procedures for the propagation of uncertainty is indicated several times by the NRC (e.g., see Quotes (YMRP4), (YMRP11)). The development of sampling-based procedures for the propagation of epistemic uncertainty in the PAs for radioactive waste disposal can be traced back to work performed in support of the NRC's original program to develop a risk assessment methodology for radioactive waste disposal. ${ }^{130-140}$ The impetus for this work was criticisms by a review committee ${ }^{141}$ that the Reactor Safety Study ${ }^{56}$ carried out by the NRC had inadequately represented the epistemic uncertainty associated with its results. This work was then continued in the NRC's MELCOR program to develop software for use in analyses of potential accidents at nuclear power plants. ${ }^{142-149}$ Subsequently, the NRC used sampling-based procedures for the propagation of epistemic uncertainty in its reassessment of results obtained in the Reactor Safety Study (i.e., in the NUREG-1150 reactor probabilistic risk assessments) ${ }^{59}, 60,150-154$ and also in its following Risk Methods Integration and Evaluation Program (RMIEP). ${ }^{155}$ In addition, the NRC has used sampling-based uncertainty and sensitivity analysis procedures in a sequence of studies involving reactor accident consequence models. ${ }^{156-159}$ Similar uncertainty propagation procedures were also used in the DOE's successful compliance certification application to the EPA for the Waste Isolation Pilot Plant. ${ }^{61,62}$

A simple example is now presented using the following definition for $D_{N}\left(\tau \mid \mathbf{e}_{D}\right)$, which is introduced solely for illustrative purposes:

$$
D_{N}\left(\tau \mid \mathbf{e}_{D}\right)=\delta\left(\tau-e_{D 1}\right)\left\{1-\exp \left[\left(e_{D 1}-\tau\right) e_{D 2}\right]\right\} e_{D 3} \exp \left(\tau e_{D 4}\right)\left\{1-\exp \left[\frac{-e_{D 5}}{1+\tau}\right]\right\}
$$

where

$$
\delta\left(\tau-e_{D 1}\right)= \begin{cases}0 & \text { if } \tau-e_{D 1} \leq 0 \\ 1 & \text { if } \tau-e_{D 1}>0\end{cases}
$$

and

$$
\mathbf{e}_{D}=\left[e_{D 1}, e_{D 2}, e_{D 3}, e_{D 4}, e_{D 5}\right]
$$

is a vector of epistemically uncertain analysis inputs with the distributions specified in Table 2. 
Table 2. Distributions Characterizing Epistemic Uncertainty in the Variables $e_{D 1}, e_{D 2}, \ldots, e_{D 5}$ Used in the Definition of $D_{N}\left(\tau \mid \mathbf{e}_{D}\right)$ in Eq. (7.9)

\begin{tabular}{cll}
\hline Variable & \multicolumn{1}{c}{ Distribution } & \multicolumn{1}{c}{ Density Function } \\
\hline$e_{D 1}$ & Uniform on [100,1000 yr] & $d_{D 1}\left(e_{D 1}\right)=1 /(1000-100)$ \\
$e_{D 2}$ & Loguniform on $\left[10^{-4}, 10^{-2} \mathrm{yr}^{-1}\right]$ & $d_{D 2}\left(e_{D 2}\right)=1 /\left[e_{D 2} \ln \left(10^{-2} / 10^{-4}\right)\right]$ \\
$e_{D 3}$ & Uniform on [0.1,1 $\mathrm{mrem} / \mathrm{yr}]$ & $d_{D 3}\left(e_{D 3}\right)=1 /(1-0.1)$ \\
$e_{D 4}$ & Loguniform on $\left[10^{-6}, 10^{-4} \mathrm{yr}^{-1}\right]$ & $d_{D 4}\left(e_{D 4}\right)=1 /\left[e_{D 4} \ln \left(10^{-4} / 10^{-6}\right)\right]$ \\
$e_{D 5}$ & Uniform on $\left[10^{3}, 10^{4} \mathrm{yr}\right]$ & $d_{D 5}\left(e_{D 5}\right)=1 /\left(10^{4}-10^{3}\right)$ \\
\hline
\end{tabular}

The expected value $E_{E}\left[D_{N}\left(\tau \mid \mathbf{e}_{D}\right)\right]$ for the function $D_{N}\left(\tau \mid \mathbf{e}_{D}\right)$ defined in Eq. (7.9) is formally given by

$$
\begin{aligned}
E_{E}\left[D_{N}\left(\tau \mid \mathbf{e}_{D}\right)\right] & =\int_{\mathcal{E D}} D_{N}\left(\tau \mid \mathbf{e}_{D}\right) d_{D}\left(\mathbf{e}_{D}\right) \mathrm{d} E D \\
& =\int_{100}^{1000} \int_{10^{-4}}^{10^{-2}} \int_{0.1}^{1} \int_{10^{-6}}^{10^{-4}} \int_{10^{3}}^{10^{4}} D_{N}\left(\tau \mid \mathbf{e}_{D}\right) \prod_{j=1}^{5} d_{D j}\left(e_{D j}\right) \prod_{j=1}^{5} \mathrm{~d} e_{D j} \\
& =\frac{\int_{100}^{1000} \int_{10^{-4}}^{10^{-2}} \int_{0.1}^{1} \int_{10^{-6}}^{10^{-4}} \int_{10^{3}}^{10^{4}}\left[D_{N}\left(\tau \mid \mathbf{e}_{D}\right) /\left(e_{D 2} e_{D 4}\right)\right] \mathrm{d} e_{D 1} \mathrm{~d} e_{D 2} \mathrm{~d} e_{D 3} \mathrm{~d} e_{D 4} \mathrm{~d} e_{D 5}}{(900)\left(\ln 10^{2}\right)(0.9)\left(\ln 10^{2}\right)(9000)} .
\end{aligned}
$$

An analogous representation holds for $p_{E}\left[D_{N}\left(\tau \mid \mathbf{e}_{D}\right) \leq D\right]$ with $D_{N}\left(\tau \mid \mathbf{e}_{D}\right)$ replaced by $\delta_{D}\left[D_{N}\left(\tau \mid \mathbf{e}_{D}\right)\right]$. Although it would be messy, the representation for $E_{E}\left[D_{N}\left(\tau \mid \mathbf{e}_{D}\right)\right]$ in Eq. (7.10) is amenable to both a closed form representation and a quadrature-based approximation. A closed form representation for $p_{E}\left[D_{N}\left(\tau \mid \mathbf{e}_{D}\right) \leq D\right]$ is difficult to obtain because of the indicator function $\underline{\delta}_{D}$ but a quadrature-based approximation is possible. As $D_{N}\left(\tau \mid \mathbf{e}_{D}\right)$ increases in complexity (e.g., involves the solution of multiple systems of partial differential equations) and $\mathbf{e}_{D}$ increases in dimensionality (e.g., involves from 10's to 100's of elements), representations of the form appearing in Eq. (7.10) become too complex to deal with directly.

As previously indicated, increasing complexity in $D_{N}\left(\tau \mid \mathbf{e}_{D}\right)$ and dimensionality in $\mathbf{e}_{D}$ results in a need to use sampling-based procedures to estimate $E_{E}\left[D_{N}\left(\tau \mid \mathbf{e}_{D}\right)\right]$ and $p_{E}\left[D_{N}\left(\tau \mid \mathbf{e}_{D}\right) \leq D\right]$ as indicated in conjunction with Eqs. (7.5) - (7.7). The results of this approach for the function $D_{N}\left(\tau \mid \mathbf{e}_{D}\right)$ in Eq. (7.9) are illustrated for a LHS

$$
\mathbf{e}_{D i}=\left[e_{D 1 i}, e_{D 2 i}, \ldots, \quad e_{D 5 i}\right], i=1,2, \ldots, n S=200,
$$

generated in consistency with the distributions defined in Table 2. The resultant 200 dose curves $D_{N}\left(\tau \mid \mathbf{e}_{D i}\right)$ for $0 \leq$ $\tau \leq 20,000$ yr appear in Fig. 1a, and the corresponding estimates for the expected dose curve $E_{E}\left[D_{N}\left(\tau \mid \mathbf{e}_{D}\right)\right]$ and associated quantile curves (i.e., 0.05, $0.5 \sim$ median, 0.95) appear in Fig. 1b. The expected dose curve in Fig. 1b is a plot of the points

$$
\left[\tau, \hat{E}_{E}\left[D_{N}\left(\tau \mid \mathbf{e}_{D}\right)\right]\right]=\left[\tau, \sum_{i=1}^{n S} D_{N}\left(\tau \mid \mathbf{e}_{D i}\right) / n S\right]
$$

for $0 \leq \tau \leq 20,000 \mathrm{yr}$, with the summation corresponding to the approximation in Eq. (7.6). 


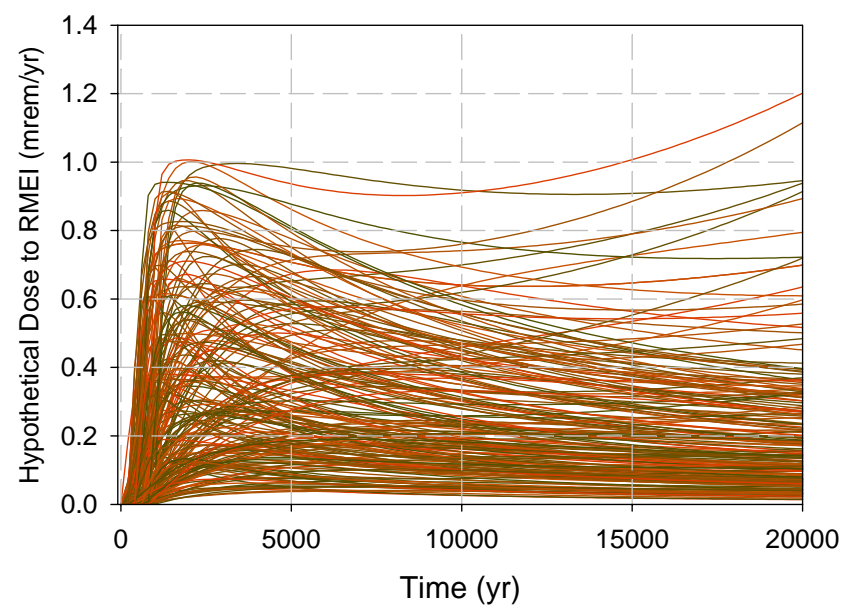

(a)

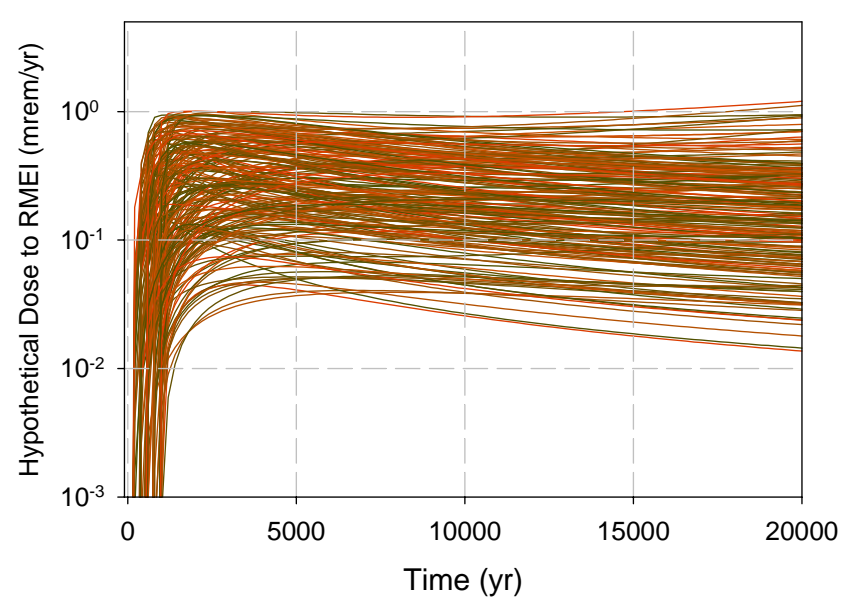

(c)

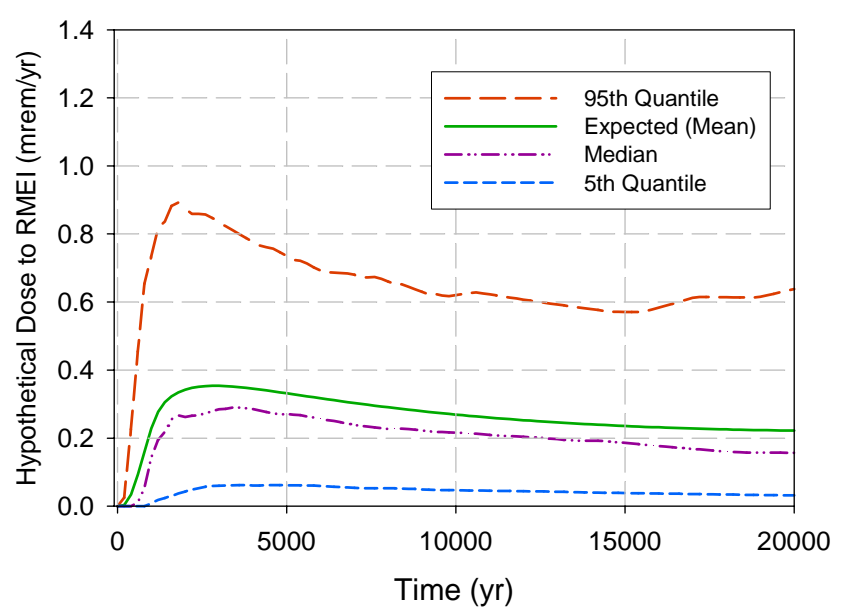

(b)

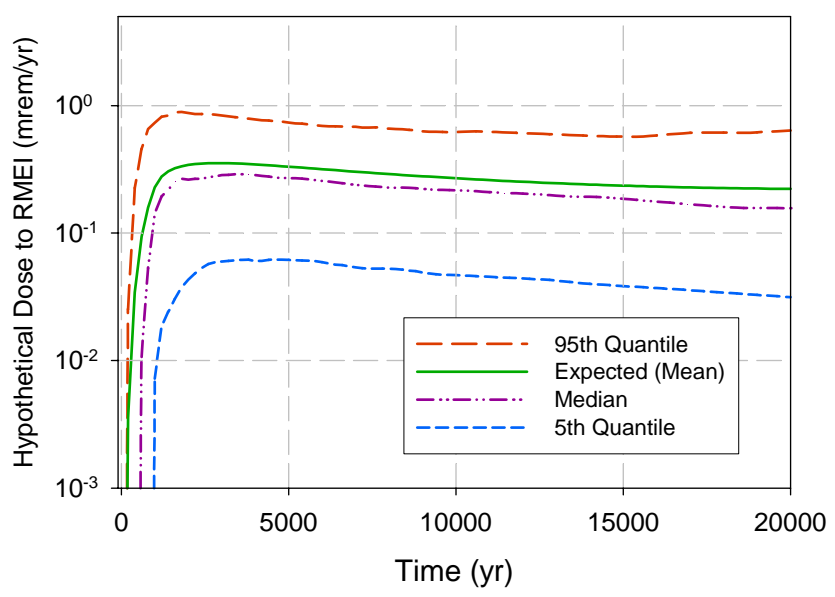

(d)

Fig. 1. Results for hypothetical dose function $D_{N}\left(\tau \mid \mathbf{e}_{D}\right)$ defined in Eq. (7.9) obtained with a LHS of size $n S=200$ : (a, c) Individual dose curves $\left[\tau, D_{N}\left(\tau \mid \mathbf{e}_{D i}\right)\right], i=1,2, \ldots, n S=200$, and (b, d) Estimated expected and quantile curves.

The quantile curves in Fig. 1b derive from the quantiles of distributions of the form illustrated in Fig. 2 for $\tau=$ 5,000 yr. Specifically, the estimated CDF in Fig. 2 is a plot of the points

$$
\left[D, \hat{p}_{E}\left[D_{N}\left(5000 \mid \mathbf{e}_{D}\right) \leq D\right]\right]=\left[D, \sum_{i=1}^{n S} \underline{\delta}_{D}\left[D_{N}\left(5000 \mid \mathbf{e}_{D i}\right)\right] / n S\right]
$$

with the summation corresponding to the approximation in Eq. (7.7). An analogous definition holds for the CCDF (see Eq. (3.5)). Quantile curves of the form illustrated in Fig. 1b are local in the sense that they are expressing the uncertainty in dose at a specific point in time as characterized by estimated distributions of the form shown in Fig. 2.

For perspective, the results in Fig. 1 are presented for both raw (i.e., untransformed) dose (Figs. 1a, 1b) and log-transformed dose (Figs. 1c, 1d). Even though the same results are being presented, plots with raw and logtransformed data can look quite different and sometimes lead to different impressions about the nature of the data. Thus, care must be used in interpreting data to avoid misimpressions that can arise from transformations used to facilitate plotting. The use of the log-transformation is common, and often essential, when the data to be plotted extend over many orders of magnitude. 


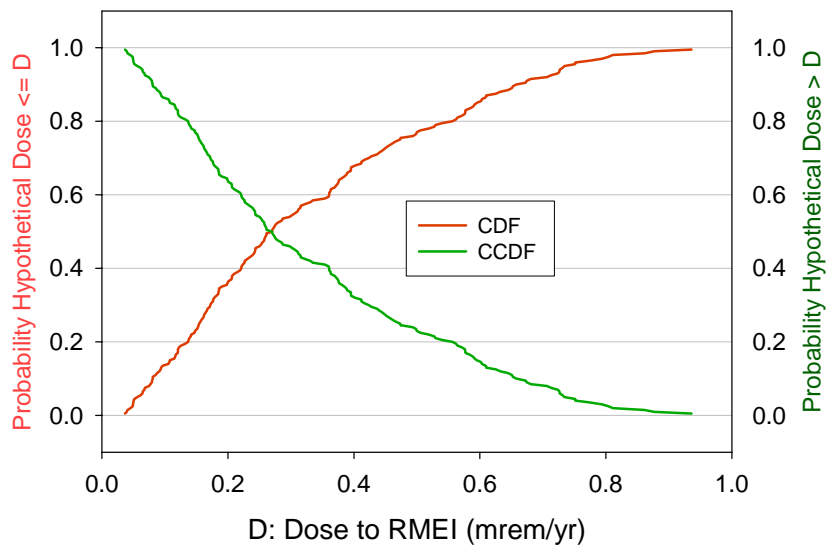

Fig. 2. Estimated CDF and CCDF for hypothetical dose function $D_{N}\left(\tau \mid \mathbf{e}_{D}\right)$ defined in Eq. (7.9) at $\tau=5000 \mathrm{yr}$ obtained with a LHS of size $n S=200$.

The dose curves in Figs. 1a and 1c and associated quantile curves in Figs. 1b and 1d are displaying the epistemic uncertainty in $D_{N}\left(\tau \mid \mathbf{e}_{D}\right)$ that derives from uncertainty in $\mathbf{e}_{D}$ as characterized by the distributions specified in Table 2. There is no epistemic uncertainty associated with the unique value for $E_{E}\left[D_{N}\left(\tau \mid \mathbf{e}_{D}\right)\right]$ as this quantity is completely determined once $D_{N}\left(\tau \mid \mathbf{e}_{D}\right)$ and the distributions associated with the elements of $\mathbf{e}_{D}$ are specified. However, the unique value for $E_{E}\left[D_{N}\left(\tau \mid \mathbf{e}_{D}\right)\right]$ is unlikely to be ascertainable in any real analysis because of the numerical approximations used in its estimation. If the numerical error in the calculation of $D_{N}\left(\tau \mid \mathbf{e}_{D}\right)$ is assumed to be negligible (e.g., the error in the numerical solution of a partial differential equation that constitutes part of $D_{N}\left(\tau \mid \mathbf{e}_{D}\right)$; see Refs. [160-162] for discussions of this form of error) and $E_{E}\left[D_{N}\left(\tau \mid \mathbf{e}_{D}\right)\right]$ is estimated with the sampling-based approach indicated in conjunction with Eq. (7.6), then the error in the estimation of $E_{E}\left[D_{N}\left(\tau \mid \mathbf{e}_{D}\right)\right]$ results from the variability in outcomes inherent in the use of samples from a population to estimate the expected value of that population. In this case, the population is the set of all possible values for $D_{N}\left(\tau \mid \mathbf{e}_{D}\right)$ that derive from the possible values for $\mathbf{e}_{D}$, and the expected value is $E_{E}\left[D_{N}\left(\tau \mid \mathbf{e}_{D}\right)\right]$.

If random sampling is used in the estimation of $E_{E}\left[D_{N}\left(\tau \mid \mathbf{e}_{D}\right)\right]$, then the normal distribution can be used to estimate a confidence interval for the estimate $\hat{E}_{E}\left[D_{N}\left(\tau \mid \mathbf{e}_{D}\right)\right]$. Specifically, the $1-\alpha$ confidence interval for $\hat{E}_{E}\left[D_{N}\left(\tau \mid \mathbf{e}_{D}\right)\right]$ is given by

$$
\begin{aligned}
\hat{E}_{E} & {\left[D_{N}\left(\tau \mid \mathbf{e}_{D}\right)\right] \pm z_{1-\alpha / 2} S E\left(\hat{E}_{E}\left[D_{N}\left(\tau \mid \mathbf{e}_{D}\right)\right]\right) } \\
& =\left[\hat{E}_{E}\left[D_{N}\left(\tau \mid \mathbf{e}_{D}\right)\right]-z_{1-\alpha / 2} S E\left(\hat{E}_{E}\left[D_{N}\left(\tau \mid \mathbf{e}_{D}\right)\right]\right), \hat{E}_{E}\left[D_{N}\left(\tau \mid \mathbf{e}_{D}\right)\right]+z_{1-\alpha / 2} S E\left(\hat{E}_{E}\left[D_{N}\left(\tau \mid \mathbf{e}_{D}\right)\right]\right)\right],
\end{aligned}
$$

where (i) $\hat{E}_{E}\left[D_{N}\left(\tau \mid \mathbf{e}_{D}\right)\right]$ is the estimated value for $E_{E}\left[D_{N}\left(\tau \mid \mathbf{e}_{D}\right)\right]$ obtained with a random sample from the epistemically uncertain values for $\mathbf{e}_{D}$ as indicated in conjunction with Eqs. (7.5) and (7.6), (ii) $\operatorname{SE}\left(\hat{E}_{E}\left[D_{N}\left(\tau \mid \mathbf{e}_{D}\right)\right]\right)$ is the standard error associated the estimation of $E_{E}\left[D_{N}\left(\tau \mid \mathbf{e}_{D}\right)\right]$ given by

$$
S E\left(\hat{E}_{E}\left[D_{N}\left(\tau \mid \mathbf{e}_{D}\right)\right]\right)=\left(\sum_{i=1}^{n S}\left\{D_{N}\left(\tau \mid \mathbf{e}_{D i}\right)-\hat{E}_{E}\left[D_{N}\left(\tau \mid \mathbf{e}_{D}\right)\right]\right\}^{2} / n S(n S-1)\right)^{1 / 2},
$$

(iii) $z_{1-\alpha / 2}$ is the $1-\alpha / 2$ quantile of the standard normal distribution, and (iv) the inequality $n S \geq 30$ holds so that, as a result of the Central Limit Theorem, $\hat{E}_{E}\left[D_{N}\left(\tau \mid \mathbf{e}_{D}\right)\right]$ will approximately follow a normal distribution under repeated estimations with different random samples of size $n S$ (see Ref. [163], Sect. 7.3). For example, if $\alpha=0.05$, then the relationship in Eq. (7.14) defines a 0.95 (or 95\%) confidence interval for $\hat{E}_{E}\left[D_{N}\left(\tau \mid \mathbf{e}_{D}\right)\right]$, which means that the construction process that lead to the interval in Eq. (7.14) will produce an interval containing the true value for $E_{E}\left[D_{N}\left(\tau \mid \mathbf{e}_{D}\right)\right]$ in 95 out of every 100 repetitions if the process is repeated a large number of times. However, it is important to recognize that the confidence interval given by Eq. (7.14) is local in the sense that it applies for a spe- 
cific value of $\tau$; in particular, joining the confidence intervals for individual values of $\tau$ does not produce a confidence interval for the curve defined in Eq. (7.12).

Because of its efficient stratification properties, it is common in analyses of complex systems to use Latin hypercube sampling rather than random sampling in the generation of the sample in Eq. (7.5). In this situation, a confidence interval for $\hat{E}_{E}\left[D_{N}\left(\tau \mid \mathbf{e}_{D}\right)\right]$ cannot be estimated as in Eq. (7.14) because the underlying assumption that a random sample is under consideration is not satisfied. However, a confidence interval for $\hat{E}_{E}\left[D_{N}\left(\tau \mid \mathbf{e}_{D}\right)\right]$ when Latin hypercube sampling is used can be estimated with a replicated sampling procedure proposed by R.L. Iman (Ref. [164]; Sect. 7, Ref. [165]).

In this approach, the analysis is replicated $n R$ times with $n R$ independently generated LHSs of size $n S$ as indicated in Eq. (7.5). This produces $n R$ estimates for $\hat{E}_{E}\left[D_{N}\left(\tau \mid \mathbf{e}_{D}\right)\right]$ of the form

$$
\hat{E}_{E r}\left[D_{N}\left(\tau \mid \mathbf{e}_{D}\right)\right]=\sum_{i=1}^{n S} D_{N}\left(\tau \mid \mathbf{e}_{D r i}\right) / n S
$$

where $\mathbf{e}_{D r i}, i=1,2, \ldots, n S$, is the LHS generated for replicate $r$. Then,

$$
\hat{E}_{E}\left[D_{N}\left(\tau \mid \mathbf{e}_{D}\right)\right]=\sum_{r=1}^{n S} \hat{E}_{E r}\left[D_{N}\left(\tau \mid \mathbf{e}_{D}\right)\right] / n R
$$

and

$$
S E\left(\hat{E}_{E}\left[D_{N}\left(\tau \mid \mathbf{e}_{D}\right)\right]\right)=\left(\sum_{r=1}^{n R}\left\{\hat{E}_{E r}\left[D_{N}\left(\tau \mid \mathbf{e}_{D}\right)\right]-\hat{E}_{E}\left[D_{N}\left(\tau \mid \mathbf{e}_{D}\right)\right]\right\}^{2} / n R(n R-1)\right)^{1 / 2}
$$

provide an additional estimate for $E_{E}\left[D_{N}\left(\tau \mid \mathbf{e}_{D}\right)\right]$ and an estimate of the standard error associated with this estimate of $E_{E}\left[D_{N}\left(\tau \mid \mathbf{e}_{D}\right)\right]$. The $t$-distribution with $n R-1$ degrees of freedom can now be used to obtain a confidence interval for the estimate $\hat{E}_{E}\left[D_{N}\left(\tau \mid \mathbf{e}_{D}\right)\right]$ for $E_{E}\left[D_{N}\left(\tau \mid \mathbf{e}_{D}\right)\right]$ in Eq. (7.17). Specifically, the $1-\alpha$ confidence interval for $\hat{E}_{E}\left[D_{N}\left(\tau \mid \mathbf{e}_{D}\right)\right]$ obtained with replicated Latin hypercube sampling is given by

$$
\begin{aligned}
& \hat{E}\left[D_{N}\left(\tau \mid \mathbf{e}_{D}\right)\right] \pm t_{1-\alpha / 2} S E\left(\hat{E}\left[D_{N}\left(\tau \mid \mathbf{e}_{D}\right)\right]\right) \\
& =\left[\hat{E}\left[D_{N}\left(\tau \mid \mathbf{e}_{D}\right)\right]-t_{1-\alpha / 2} S E\left(\hat{E}\left[D_{N}\left(\tau \mid \mathbf{e}_{D}\right)\right]\right), \hat{E}\left[D_{N}\left(\tau \mid \mathbf{e}_{D}\right)\right]+t_{1-\alpha / 2} S E\left(\hat{E}\left[D_{N}\left(\tau \mid \mathbf{e}_{D}\right)\right]\right)\right],
\end{aligned}
$$

where (i) $\hat{E}_{E}\left[D_{N}\left(\tau \mid \mathbf{e}_{D}\right)\right]$ and $S E\left(\hat{E}_{E}\left[D_{N}\left(\tau \mid \mathbf{e}_{D}\right)\right]\right)$ are defined in Eqs. (7.17) and (7.18) and (ii) $t_{1-\alpha / 2}$ is the $1-\alpha / 2$ quantile of the $t$-distribution with $n R-1$ degrees of freedom (e.g., $t_{1-\alpha / 2}=2.776$ for $\alpha=0.05$ and $n R=5$ ). As indicated for the confidence interval in Eq. (7.14) obtained with random sampling, the confidence interval in Eq. (7.19) obtained with Latin hypercube sampling also applies locally rather than globally.

The use of replicated Latin hypercube sampling in the assessment of numerical stability is illustrated with the function $D_{N}\left(\tau \mid \mathbf{e}_{D}\right)$ defined in Eq. (7.9). Specifically, $n R=5$ replicated LHSs of size $n S=100$ are generated from the possible values for $\mathbf{e}_{D}$ in consistency with the distributions defined in Table 2. The resultant five sets of mean and quantile curves are presented in Fig. 3a. Further, the five estimated distributions for $p_{E}\left[D_{N}\left(5000 \mid \mathbf{e}_{D}\right)<D\right]$ defined in Eq. (7.7) are presented in Fig. 3c, and the confidence intervals for $\bar{D}_{N}(\tau)$ defined as indicated in Eq. (7.19) are presented in Fig. 3e. 


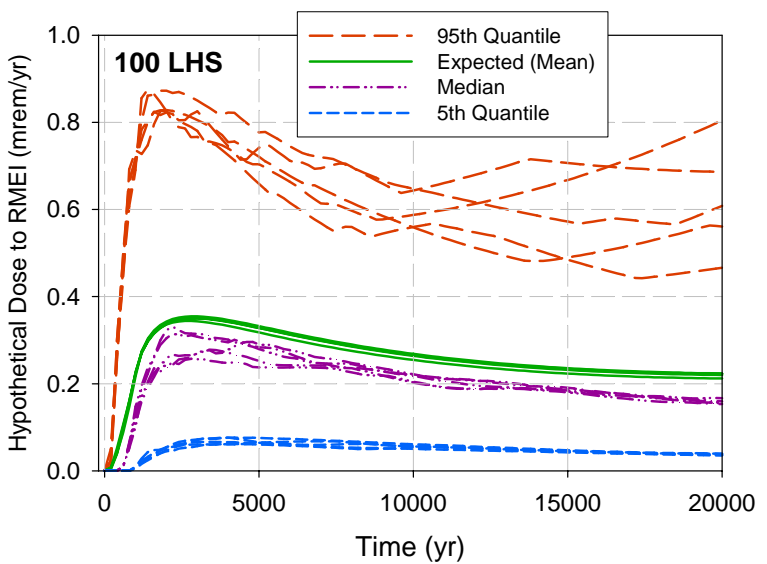

(a)

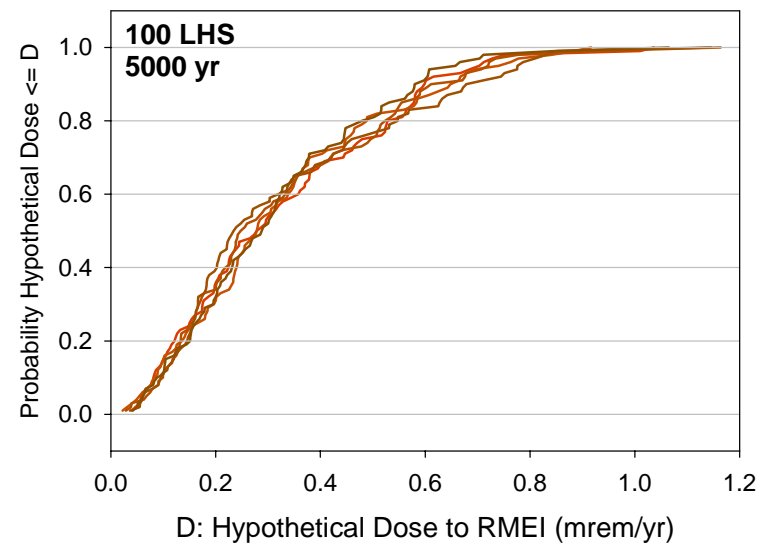

(c)

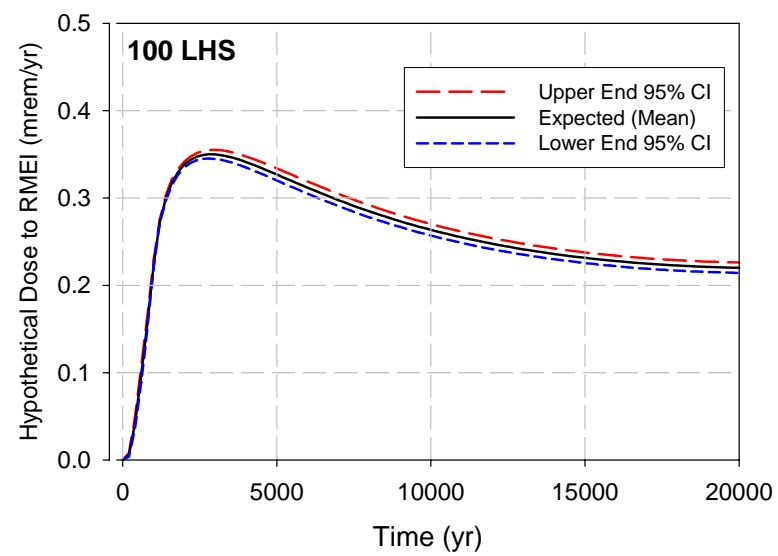

(e)

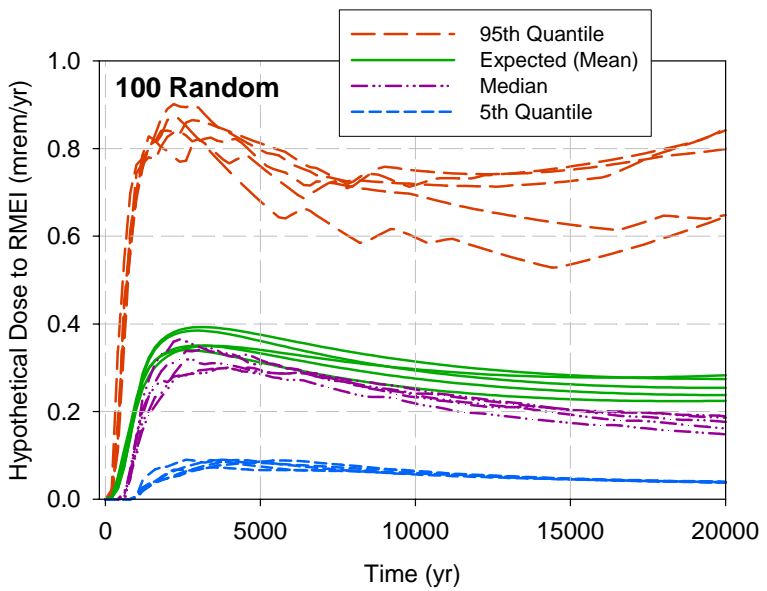

(b)

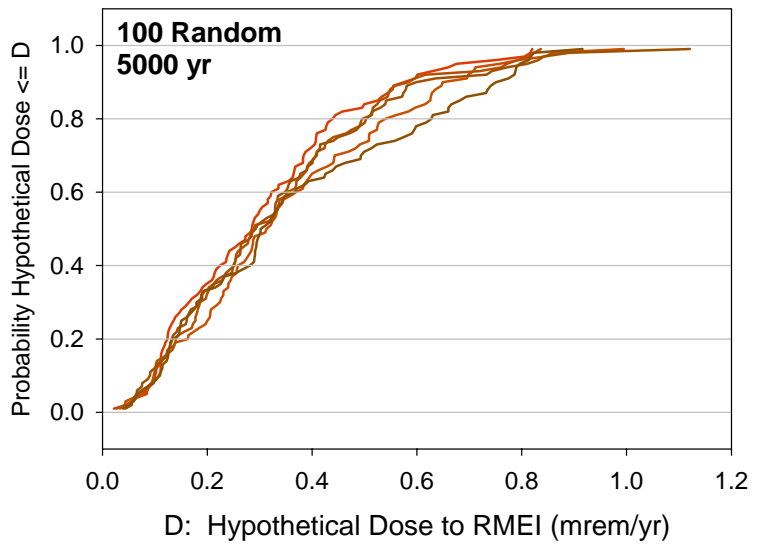

(d)

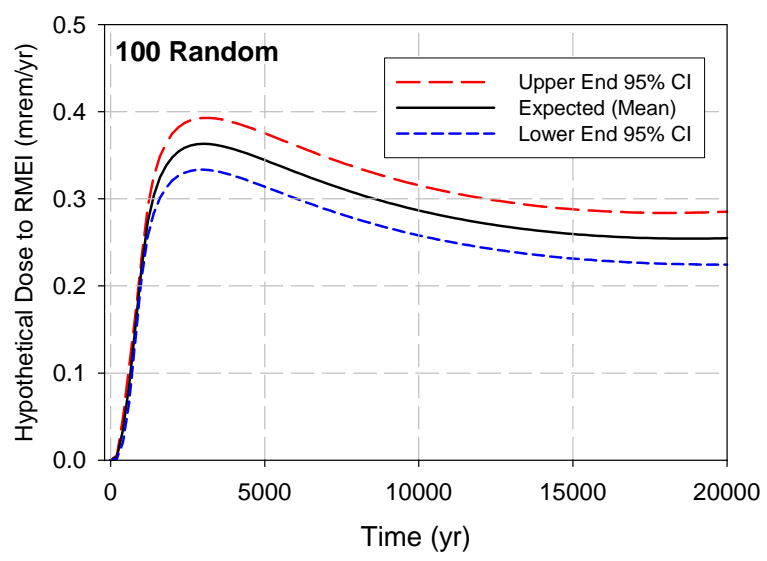

(f)

Fig. 3. Results for hypothetical dose function $D_{N}\left(\tau \mid \mathbf{e}_{D}\right)$ defined in Eq. (7.9) obtained with $n R=5$ replicated samples of size $n S=100$ with Latin hypercube sampling (a, c, e) and random sampling (b, d, f): (a, b) Estimated expected and quantile curves for individual replicates, (c, d) Estimated CDFs for dose at $5000 \mathrm{yr}$ for individual replicates, and (e, f) 95\% confidence intervals (CIs) for estimated expected values. 
For comparison, the analysis was also performed with $n R=5$ replicated random samples of size $n S=100$ (Figs. 3b, 3d, 3f). As comparison of the results obtained with Latin hypercube sampling (Figs. 3a, 3c, 3e) and random sampling (Figs. 3b, 3d, 3f) shows, the use of Latin hypercube sampling is producing more stable results than the use of random sampling. Because of this stability, Latin hypercube sampling is usually preferred over random sampling when performing uncertainty and sensitivity analyses of computationally demanding models.

One of the acceptance criteria specified by the NRC is "A sufficient number of realizations has been obtained ... to ensure that the results of the calculation are numerically stable” (see Quote (YMRP4)). An additional acceptance criterion is "the annual dose curve includes confidence intervals (e.g., $95^{\text {th }}$ and $5^{\text {th }}$ percentile) to represent the uncertainty in the dose calculations" (see Quote (YMRP5)). Presumably, these criteria apply to dose curves that involve both nominal and disturbed conditions (e.g., see Quotes (YMRP2) and (YMRP3)). However, it is interesting at this point to examine possible implications of these criteria with respect to the nominal dose results $D_{N}\left(\tau \mid \mathbf{e}_{D}\right)$ and $E_{E}\left[D_{N}\left(\tau \mid \mathbf{e}_{D}\right)\right]$. The criterion in Quote (YMRP4) relates to numerical stability. It is this type of stability that is being assessed by the confidence intervals defined in Eqs. (7.14) and (7.19) and illustrated in Figs. 3e and 3f. Specifically, the indicated confidence intervals are providing a representation of the numerical error in a sampling-based estimate for $E_{E}\left[D_{N}\left(\tau \mid \mathbf{e}_{D}\right)\right]$. In contrast, the criterion in Quote (YMRP5) seems to relate to uncertainty in the epistemic sense, which is the type of uncertainty that is being characterized by the quantile curves in Figs. $1 \mathrm{~b}$ and $1 \mathrm{~d}$. Specifically, the confidence intervals (i.e., quantile curves) in Figs. $1 \mathrm{~b}$ and 1d characterize the uncertainty in the possible values for $D_{N}\left(\tau \mid \mathbf{e}_{D}\right)$ that derives from epistemic uncertainty with respect to the appropriate value to use for $\mathbf{e}_{D}$.

An alternative interpretation is that both criteria relate to the assessment of sampling error as quantified by confidence intervals of the form shown in Eqs. (7.14) and (7.19). However, under this interpretation, the effects of epistemic uncertainty are removed from consideration. With regard to the preceding, the reader is emphatically reminded that uncertainty in the sense of numerical error and uncertainty in the sense of lack of knowledge about values for analysis inputs are entirely different aspects of an analysis. In a large analysis for a complex system, the uncertainty in the final results is more likely to be dominated by epistemic uncertainty than by numerical error. Indeed, if this is not the case, then the numerical implementation of the analysis is not appropriately converged.

The discussions of the implications of epistemic uncertainty here and elsewhere in this presentation are predicated on the assumption that the analysis under consideration has undergone appropriate verification and validation, with the result that the analysis is error free. In the preceding, verification designates obtaining assurance that an analysis and its associated models are correctly implemented, and validation designates obtaining assurance that the models used in an analysis appropriately represent the physical processes under consideration. The selection and implementation of appropriate verification and validation procedures is an important and challenging part of any large analysis, $160-162,166-172$ but is outside the primary focus of this presentation. However, certain connections with verification and validation are present. Specifically, the stability results presented in connection with Eqs. (7.14) and (7.19) constitute a special type of numerical verification; sensitivity analyses of mappings between analysis inputs and analysis results of the form shown in Eq. (7.8) provide another form of analysis verification; and model validation studies can be expected to influence the uncertainty distributions assigned to many analysis inputs. 


\section{Calculation of Expected Value and Display of Epistemic Uncertainty for $E_{A}\left[D_{D}\left(\tau \mid \mathbf{a}, \mathbf{e}_{D}\right) \mid \mathbf{e}_{A}\right]$}

The calculation of expected value and the display of epistemic uncertainty is now considered for $E_{A}\left[D_{D}(\tau \mid \mathbf{a}\right.$, $\left.\left.\mathbf{e}_{D}\right) \mid \mathbf{e}_{A}\right]$, which is the expected dose over aleatory uncertainty conditional on a specific value for the epistemically uncertain vector $\mathbf{e}=\left[\mathbf{e}_{A}, \mathbf{e}_{D}\right]$ defined in Eq. (6.6). In concept, $E_{A}\left[D_{D}\left(\tau \mid \mathbf{a}, \mathbf{e}_{D}\right) \mid \mathbf{e}_{A}\right]$ could be either of the expected doses $E_{A}\left[D_{I}\left(\tau \mid \mathbf{a}, \mathbf{e}_{D}\right) \mid \mathbf{e}_{A}\right]$ and $E_{A}\left[D_{S}\left(\tau \mid \mathbf{a}, \mathbf{e}_{D}\right) \mid \mathbf{e}_{A}\right]$ defined in Eqs. (6.7) and (6.8).

Epistemic uncertainty with respect to the appropriate value to use for $\mathbf{e}$ results in many possible values for $E_{A}\left[D_{D}\left(\tau \mid \mathbf{a}, \mathbf{e}_{D}\right) \mid \mathbf{e}_{A}\right]$, with a different value for $E_{A}\left[D_{D}\left(\tau \mid \mathbf{a}, \mathbf{e}_{D}\right) \mid \mathbf{e}_{A}\right]$ resulting for each possible value for $\mathbf{e}$. These possible values for $E_{A}\left[D_{D}\left(\tau \mid \mathbf{a}, \mathbf{e}_{D}\right) \mid \mathbf{e}_{A}\right]$ have a distribution that derives from the distributions that characterize the epistemic uncertainty associated with the elements of $\mathbf{e}$. Specifically, the distribution for $E_{A}\left[D_{D}\left(\tau \mid \mathbf{a}, \mathbf{e}_{D}\right) \mid \mathbf{e}_{A}\right]$ represents the epistemic uncertainty in the expected dose that derives from aleatory uncertainty (i.e., from the assumed randomness of disruptive events that could, but may not, occur at some time in the future).

The distribution of possible values for $E_{A}\left[D_{D}\left(\tau \mid \mathbf{a}, \mathbf{e}_{D}\right) \mid \mathbf{e}_{A}\right]$ has an expected value that can be formally represented by

$$
\begin{aligned}
E_{E}\left\{E_{A}\left[D_{D}\left(\tau \mid \mathbf{a}, \mathbf{e}_{D}\right) \mid \mathbf{e}_{A}\right]\right\} & =\int_{\mathcal{E}} E_{A}\left[D_{D}\left(\tau \mid \mathbf{a}, \mathbf{e}_{D}\right) \mid \mathbf{e}_{A}\right] d_{E}(\mathbf{e}) \mathrm{d} E \\
& =\int_{\mathcal{E}} \int_{a}^{\tau} \int_{\mathcal{P}} D_{D}\left(\tau \mid t, \mathbf{p}, \mathbf{e}_{D}\right) \lambda_{D} d_{P}\left(\mathbf{p} \mid \mathbf{e}_{A}\right) d_{E}(\mathbf{e}) \mathrm{d} P \mathrm{~d} t \mathrm{~d} E
\end{aligned}
$$

as previously indicated in Eq. (6.16). Further, the CDF that characterizes the epistemic uncertainty in the possible values for $E_{A}\left[D_{D}\left(\tau \mid \mathbf{a}, \mathbf{e}_{D}\right) \mid \mathbf{e}_{A}\right]$ is formally defined by

$$
\begin{aligned}
p_{E}\left\{E_{A}\left[D_{D}\left(\tau \mid \mathbf{a}, \mathbf{e}_{D}\right) \mid \mathbf{e}_{A}\right] \leq D\right\}= & \text { epistemic probability of an expected dose } E_{A}\left[D_{D}\left(\tau \mid \mathbf{a}, \mathbf{e}_{D}\right) \mid \mathbf{e}_{A}\right] \text { over } \\
& \text { aleatory uncertainty less than } D \text { at time } \tau \\
= & \int_{\mathcal{E}} \underline{\delta}_{D}\left\{E_{A}\left[D_{D}\left(\tau \mid \mathbf{a}, \mathbf{e}_{D}\right) \mid \mathbf{e}_{A}\right]\right\} d_{E}(\mathbf{e}) \mathrm{d} E \\
= & \int_{\mathcal{E}} \underline{\delta}_{D}\left\{\int_{a}^{\tau} \int_{\mathcal{E}} D_{D}\left(\tau \mid t, \mathbf{p}, \mathbf{e}_{D}\right) \lambda_{D} d_{P}\left(\mathbf{p} \mid \mathbf{e}_{A}\right) \mathrm{d} P \mathrm{~d} t\right\} d_{E}(\mathbf{e}) \mathrm{d} E
\end{aligned}
$$

where $\underline{\delta}_{D}$ is the indicator function defined in Eq. (7.3). Given that the determination of $E_{E}\left\{E_{A}\left[D_{D}\left(\tau \mid \mathbf{a}, \mathbf{e}_{D}\right) \mid \mathbf{e}_{A}\right]\right\}$ and $p_{E}\left\{E_{A}\left[D_{D}\left(\tau \mid \mathbf{a}, \mathbf{e}_{D}\right) \mid \mathbf{e}_{A}\right]<D\right\}$ involves the evaluation of three iterated integrals of which two are over what are likely to be high dimensional spaces (i.e., the spaces $\mathcal{E}$ and $\mathscr{P}$ ), the use of quadrature-based methods in this determination is unlikely to be practicable. Thus, some other evaluation strategy must be sought.

This section considers three possible computational strategies for the determination of $E_{E}\left\{E_{A}\left[D_{D}(\tau \mid \mathbf{a}\right.\right.$, $\left.\left.\left.\mathbf{e}_{D}\right) \mid \mathbf{e}_{A}\right]\right\}$ : (i) Strategy 1, sample from $\mathcal{E}$ and numerically evaluate $E_{A}\left[D_{D}\left(\tau \mid \mathbf{a}, \mathbf{e}_{D}\right) \mid \mathbf{e}_{A}\right]$ (Sect. 8.1), (ii) Strategy 2, sample from $\mathcal{E} \times \mathcal{P}$ and numerically evaluate the integral over time (Sect. 8.2), and (ii) Strategy 3, sample from $\mathcal{E} \times$ $\mathscr{P} \times[a, b]$ with importance sampling on time (Sect. 8.3). Of these strategies, only Strategy 1 has a structure that also allows the determination of $p_{E}\left\{E_{A}\left[D_{D}\left(\tau \mid \mathbf{a}, \mathbf{e}_{D}\right) \mid \mathbf{e}_{A}\right]<D\right\}$.

As for the nominal scenario class in Sect. 7, it is beneficial to introduce a simple function that can be used to illustrate the computational procedures under consideration. This hypothetical dose function is defined by

$$
\begin{aligned}
D_{D}\left(\tau \mid t, \mathbf{p}, \mathbf{e}_{D}\right)= & \text { incremental dose (mrem/yr) to RMEI at time } \tau \text { resulting from a disruptive event at time } t \\
& \text { with properties defined by the vector } \mathbf{p} \text { and conditional on the vector } \mathbf{e}_{D} \text { of values for } \\
& \text { epistemically uncertain analysis inputs }
\end{aligned}
$$


Table 3. Distributions Characterizing Epistemic Uncertainty in the Variables $e_{D 6}, e_{D 7}$ and $e_{D 8}$ Used in the Definition of $D_{N}\left(\tau \mid t, \mathbf{p}, \mathbf{e}_{D}\right)$ in Eq. (8.3) (see Table 2 for distributions for $e_{D 1}, e_{D 2}, \ldots, e_{D 5}$ ) and in the Variables $e_{A 1}, e_{A 2}$ and $e_{A 3}$ Used in the Characterization of Aleatory Uncertainty in $a_{1}, a_{2}$ and $t$

\begin{tabular}{cll}
\hline Variable & \multicolumn{1}{c}{ Distribution } & \multicolumn{1}{c}{ Density Function } \\
\hline$e_{D 6}$ & Uniform on [2, 25 mrem/yr] & $d_{D 6}\left(e_{D 6}\right)=1 /(25-2)$ \\
$e_{D 7}$ & Loguniform on $\left[10^{-7}, 10^{-5} \mathrm{yr}^{-1}\right]$ & $d_{D 7}\left(e_{D 7}\right)=1 /\left[e_{D 7} \ln \left(10^{-5} / 10^{-7}\right)\right]$ \\
$e_{D 8}$ & Uniform on [15, 45 mrem/yr] & $d_{D 8}\left(e_{D 8}\right)=1 /(45-15)$ \\
$e_{D 9}$ & Loguniform on $\left[10^{-4}, 10^{-3} \mathrm{yr}^{-1}\right]$ & $d_{D 9}\left(e_{D 9}\right)=1 /\left[e_{D 9} \ln \left(10^{-3} / 10^{-4}\right)\right]$ \\
$e_{A 1}$ & Uniform on [log(0.5), log(2.0)] & $d_{A 1}\left(e_{A 1}\right)=1 / \log (2.0 / 0.5)$ \\
$e_{A 2}$ & Uniform on [0.3, 1] & $d_{A 2}\left(e_{A 2}\right)=1 /(1.0-0.3)$ \\
$e_{A 3}=\lambda_{D}$ & Loguniform on $\left[10^{-6}, 10^{-4} \mathrm{yr}^{-1}\right]$ & $d_{A 3}\left(e_{A 3}\right)=1 /\left[e_{A 3} \ln \left(10^{-4} / 10^{-6}\right)\right]$ \\
\hline
\end{tabular}

$$
\begin{aligned}
= & \delta(\tau-t)\left\{1-\exp \left[(t-\tau) e_{D 2}\right]\right\} \\
& \times\left\{e_{D 3} a_{1} \exp \left[(\tau-t) e_{D 4}\right]+e_{D 6} a_{2} \exp \left[(\tau-t) e_{D 7}\right]+e_{D 8} \exp \left(-t e_{D 9}\right)\right\} \\
& \times\left\{1-\exp \left[-e_{D 5} /(1+\tau-t)\right]\right\}
\end{aligned}
$$

for $a \leq \tau, t \leq b$, with $\delta(\tau-t)$ defined in conjunction with Eq. (7.9), [a, $b]=[0,20,000 \mathrm{yr}], \mathbf{p}=\left[a_{1}, a_{2}\right]$ and $\mathbf{e}_{D}=\left[e_{D 1}\right.$, $\left.e_{D 2}, \ldots, e_{D 9}\right]$. The inclusion of the factor $\delta(\tau-t)$ results in $D_{D}\left(\tau \mid t, \mathbf{p}, \mathbf{e}_{D}\right)=0$ for $\tau \leq t$. Of the variables contained in $\mathbf{e}_{D}$, (i) $\mathbf{e}_{D 1}$ only affects $D_{N}\left(\tau \mid e_{D}\right)$ defined in Eq. (5.10), (ii) $e_{D 2}, e_{D 3}, e_{D 4}$ and $e_{D 5}$ affect both $D_{N}\left(\tau \mid \mathbf{e}_{D}\right)$ and $D_{D}(\tau \mid t$, $\mathbf{p}$, $\mathbf{e}_{D}$ ), and (iii) $e_{D 6}, e_{D 7}, e_{D 8}$ and $e_{D 9}$ only affect $D_{D}\left(\tau \mid t, \mathbf{p}, \mathbf{e}_{D}\right)$. This definition of $\mathbf{e}_{D}$ is made to emphasize that, in a real analysis, some epistemically uncertain variables will relate only to nominal conditions, while others will relate to both nominal and disturbed conditions and yet others will relate to only disturbed conditions. The distributions for $e_{D 1}, e_{D 2}$, $\ldots, e_{D 5}$ are given in Table 2, and the distributions for $e_{D 6}, e_{D 7}, e_{D 8}, e_{D 9}$ are given in Table 3.

In this simple example, the vector $\mathbf{a}$ of aleatory variables associated with $D_{D}\left(\tau \mid t, \mathbf{p}, \mathbf{e}_{D}\right)$ in Eq. (6.1) is

$$
\mathbf{a}=[t, \mathbf{p}]=\left[t, a_{1}, a_{2}\right] \text {. }
$$

The variable $a_{1}$ is assumed to have a logtriangular distribution on $[0.5,20]$ with an epistemically uncertain mode $e_{A 1}$; the variable $a_{2}$ is assumed to have a triangular distribution on $[0.3,3]$ with an epistemically uncertain mode $e_{A 2}$; and the occurrence time $t$ has a distribution that follows from the epistemically uncertain occurrence rate $e_{A 3}=$ $\lambda_{D}$ for the particular type of distribution under consideration. More specifically, $a_{1}$ and $a_{2}$ have distributions defined by the density functions

$$
\begin{aligned}
d_{A 1}\left(a_{1} \mid e_{A 1}\right) & =\left[2 \ln \left(a_{1} / 0.5\right)\right] /\left[a_{1} \ln (20 / 0.5) \ln \left(e_{A 1} / 0.5\right)\right] & & \text { for } 0.5 \leq a_{1} \leq e_{A 1} \\
& =\left[2 \ln \left(20 / a_{1}\right)\right] /\left[a_{1} \ln (20 / 0.5) \ln \left(20 / e_{A 1}\right)\right] & & \text { for } e_{A 1} \leq a_{1} \leq 20 \\
& =0 & & \text { otherwise }
\end{aligned}
$$

and 


$$
\begin{aligned}
d_{A 2}\left(a_{2} \mid e_{A 2}\right) & =\left[2\left(a_{2}-0.3\right)\right] /\left[\left(e_{A 2}-0.3\right)(3-0.3)\right] & & \text { for } 0.3 \leq a_{2} \leq e_{A 2} \\
& =\left[2\left(3-a_{2}\right)\right] /\left[\left(3-e_{A 2}\right)(3-0.3)\right] & & \text { for } e_{A 2} \leq a_{2} \leq 3 \\
& =0 & & \text { otherwise, }
\end{aligned}
$$

respectively; the set $\mathscr{P}$ corresponds to the rectangle $[0.5,20] \times[0.3,3]$; and the density function $d_{P}(\mathbf{p})$ is given by

$$
d_{P}\left(\mathbf{p} \mid \mathbf{e}_{A}\right)=d_{A 1}\left(a_{1} \mid e_{A 1}\right) d_{A 2}\left(a_{2} \mid e_{A 2}\right)
$$

for $\mathbf{p}=\left[a_{1}, a_{2}\right] \in \mathscr{P}$. Further, the distribution associated with $t$ that results from $e_{A 3}=\lambda_{D}$ has been incorporated into the definition of $E_{A}\left[D\left(\tau \mid \mathbf{a}, \mathbf{e}_{D}\right) \mathbf{e}_{A}\right]$ through the derivation of the defining integral for $E_{A}\left[D\left(\tau \mid \mathbf{a}, \mathbf{e}_{D}\right) \mathbf{e}_{A}\right]$ in Eq. (6.6). The distributions characterizing the epistemic uncertainty in $e_{A 1}, e_{A 2}$ and $e_{A 3}$ are given in Table 3.

\subsection{Strategy 1: Sample from $\mathcal{E}$ and Numerically Evaluate $E_{A}\left[D\left(\tau \mid \mathrm{a}, \mathrm{e}_{D}\right) \mid \mathrm{e}_{A}\right]$}

This strategy involves generating a sample $\mathbf{e}_{i}=\left[\mathbf{e}_{A i}, \mathbf{e}_{D i}\right], i=1,2, \ldots, n S$, from $\mathcal{E}$ and then numerically evaluating $E_{A}\left[D_{D}\left(\tau \mid \mathbf{a}, \mathbf{e}_{D i}\right) \mid \mathbf{e}_{A i}\right]$ for $a \leq \tau \leq b$ and $i=1,2, \ldots, n S$. With this strategy, the expected value $E_{E}\left\{E_{A}\left[D_{D}(\tau \mid \mathbf{a}\right.\right.$, $\left.\left.\left.\mathbf{e}_{D}\right) \mid \mathbf{e}_{A}\right]\right\}$ over epistemic uncertainty for $E_{A}\left[D_{D}\left(\tau \mid \mathbf{a}, \mathbf{e}_{D}\right) \mid \mathbf{e}_{A}\right]$ and the associated probability $p_{E}\left\{E_{A}\left[D_{D}\left(\tau \mid \mathbf{a}, \mathbf{e}_{D}\right) \mid \mathbf{e}_{A}\right]\right.$ $\leq D\}$ are approximated by

$$
\hat{E}_{E}\left\{E_{A}\left[D_{D}\left(\tau \mid \mathbf{a}, \mathbf{e}_{D}\right) \mid \mathbf{e}_{A}\right]\right\}=\sum_{i=1}^{n S} \hat{E}_{A}\left[D_{D}\left(\tau \mid \mathbf{a}, \mathbf{e}_{D i}\right) \mid \mathbf{e}_{A i}\right] / n S
$$

and

$$
\hat{p}_{E}\left\{E_{A}\left[D_{D}\left(\tau \mid \mathbf{a}, \mathbf{e}_{D}\right) \mid \mathbf{e}_{A}\right] \leq D\right\}=\sum_{i=1}^{n S} \underline{\delta}_{D}\left\{\hat{E}_{A}\left[D_{D}\left(\tau \mid \mathbf{a}, \mathbf{e}_{D i}\right) \mid \mathbf{e}_{A i}\right]\right\} / n S,
$$

where an approximation $\hat{E}_{A}\left[D_{D}\left(\tau \mid \mathbf{a}, \mathbf{e}_{D i}\right) \mid \mathbf{e}_{A i}\right]$ to $E_{A}\left[D_{D}\left(\tau \mid \mathbf{a}, \mathbf{e}_{D i}\right) \mid \mathbf{e}_{A i}\right]$ is indicated because an exact evaluation of the integral in Eq. (6.6) that formally defines $E_{A}\left[D_{D}\left(\tau \mid \mathbf{a}, \mathbf{e}_{D i}\right) \mid \mathbf{e}_{A i}\right]$ is unlikely to be possible in any real analysis.

The implementation of Strategy 1 is now illustrated with the function $D_{D}\left(\tau \mid t, \mathbf{p}, \mathbf{e}_{D}\right)$ and associated definitions for $\mathbf{p}, \mathbf{e}_{A}$ and $\mathbf{e}_{D}$ introduced in conjunction with Eq. (8.3). This illustration initially uses a LHS

$$
\mathbf{e}_{i}=\left[\mathbf{e}_{A i}, \mathbf{e}_{D i}\right]=\left[e_{A 1 i}, e_{A 2 i}, e_{A 3 i}, e_{D 1 i} e_{D 2 i}, \ldots, e_{D 9 i}\right], i=1,2, \ldots, n S,
$$

of size $n S=100$ from the set $\mathcal{E}$ of possible values for $\mathbf{e}$ generated in consistency with the distributions characterizing epistemic uncertainty specified in Tables 2 and 3. In turn, $e_{A 1 i}$ and $e_{A 2 i}$ define distributions for $a_{1}$ and $a_{2}$ characterized by the density functions $d_{A 1}\left(a_{1} \mid e_{A 1 i}\right)$ and $d_{A 2}\left(a_{2} \mid e_{A 2 i}\right)$ given in Eqs. (8.5) and (8.6), and $e_{A 3 i}=\lambda_{D i}$ defines the distribution for $t$ that is incorporated into the defining integral for $E_{A}\left[D_{D}\left(\tau \mid \mathbf{a}, \mathbf{e}_{D i}\right) \mid \mathbf{e}_{A i}\right]$.

In this example, $E_{A}\left[D_{D}\left(\tau \mid \mathbf{a}, \mathbf{e}_{D i}\right) \mid \mathbf{e}_{A i}\right]$ is given by

$$
\begin{aligned}
E_{A}\left[D_{D}\left(\tau \mid \mathbf{a}, \mathbf{e}_{D i}\right) \mid \mathbf{e}_{A i}\right] & =\int_{a}^{\tau} \int_{\mathcal{P}} D_{D}\left(\tau \mid t, \mathbf{p}, \mathbf{e}_{D i}\right) \lambda_{D i} d_{P}\left(\mathbf{p} \mid \mathbf{e}_{A i}\right) \mathrm{d} P \mathrm{~d} t \\
& =\int_{a}^{\tau} \int_{0.5}^{20} \int_{0.3}^{3} D_{D}\left(\tau \mid t, a_{1}, a_{2}, \mathbf{e}_{D i}\right) \lambda_{D i} d_{A 1}\left(a_{1} \mid e_{A 1 i}\right) d_{A 2}\left(a_{2} \mid e_{A 2 i}\right) \mathrm{d} a_{2} \mathrm{~d} a_{1} \mathrm{~d} t \\
& =\int_{a}^{b} \int_{0.5}^{20} \int_{0.3}^{3} D_{D}\left(\tau \mid t, a_{1}, a_{2}, \mathbf{e}_{D i}\right) \lambda_{D i} d_{A 1}\left(a_{1} \mid e_{A 1 i}\right) d_{A 2}\left(a_{2} \mid e_{A 2 i}\right) \mathrm{d} a_{2} \mathrm{~d} a_{1} \mathrm{~d} t,
\end{aligned}
$$


where the final equality is introduced for notational convenience and results because $D_{D}\left(\tau \mid t, a_{1}, a_{2}, \mathbf{e}_{D i}\right)=0$ for $\tau<$ $t$. The preceding representation for $E_{A}\left[D_{D}\left(\tau \mid \mathbf{a}, \mathbf{e}_{D i}\right) \mid \mathbf{e}_{A i}\right]$ must be determined for each element $\mathbf{e}_{i}$ of the LHS in Eq. (8.10). For this example, a closed form representation for $E_{A}\left[D_{D}\left(\tau \mid \mathbf{a}, \mathbf{e}_{D i}\right) \mid \mathbf{e}_{A i}\right]$ could be determined with sufficient effort. However, in a real analysis this is unlikely to be possible; rather, an approximation $\hat{E}_{A}\left[D_{D}\left(\tau \mid \mathbf{a}, \mathbf{e}_{D i}\right) \mid \mathbf{e}_{A i}\right]$ to $E_{A}\left[D_{D}\left(\tau \mid \mathbf{a}, \mathbf{e}_{D i}\right) \mid \mathbf{e}_{A i}\right]$ would have to be determined and used in the relations in Eqs. (8.8) and (8.9) to estimate $E_{E}\left\{E_{A}\left[D_{D}\left(\tau \mid \mathbf{a}, \mathbf{e}_{D}\right) \mid \mathbf{e}_{A}\right]\right\}$ and $p_{E}\left\{E\left[D_{D}\left(\tau \mid \mathbf{a}, \mathbf{e}_{D}\right) \mid \mathbf{e}_{A}\right] \leq D\right\}$.

For this example, a quadrature-based approach is used to estimate $E_{A}\left[D_{D}\left(\tau \mid \mathbf{a}, \mathbf{e}_{D i}\right) \mid \mathbf{e}_{A i}\right]$; specifically,

$$
E_{A}\left[D\left(\tau \mid \mathbf{a}, \mathbf{e}_{D i}\right) \mid \mathbf{e}_{A i}\right] \cong \sum_{j=1}^{n T} \sum_{k=1}^{n A 1} \sum_{l=1}^{n A 2} m_{i j k l} \lambda_{D i} \Delta a_{2 l} \Delta a_{1 k} \Delta t_{j}
$$

where

$$
\begin{array}{ll}
\Delta t_{j}=t_{j}-t_{j-1} & \text { with } t_{j}=a+j(b-a) / n T \text { for } j=0,1, \ldots, n T, \\
\Delta a_{1 k}=a_{1 k}-a_{1, k-1} & \text { with } a_{1 k}=0.5(20 / 0.5)^{k / n A 1} \text { for } k=0,1, \ldots, n A 1, \\
\Delta a_{2 l}=a_{2 l}-a_{2, l-1} \quad \text { with } a_{2 l}=0.3+l(3-0.3) / n A 2 \text { for } l=0,1, \ldots, n A 2,
\end{array}
$$

and

$$
m_{i j k l}=\sum_{r=j-1}^{j} \sum_{s=k-1}^{k} \sum_{t=l-1}^{l} D_{D}\left(\tau \mid t_{r}, a_{1 s}, a_{2 t}, \mathbf{e}_{D i}\right) d_{A 1}\left(a_{1 s} \mid e_{A 1 i}\right) d_{A 2}\left(a_{2 t} \mid e_{A 2 i}\right) / 8
$$

is the average of $D_{D}\left(\tau \mid t, a_{1}, a_{2}, \mathbf{e}_{D i}\right) d_{A 1}\left(a_{1} \mid e_{A 1 i}\right) d_{A 2}\left(e_{2} \mid e_{A 2 i}\right)$ over the eight corners of the rectangular solid [ $\left.t_{j-1}, t_{j}\right]$ $\times\left[a_{1, k-1}, a_{1 k}\right] \times\left[a_{2, l-1}, a_{2 l}\right]$. As an aside, the definition of $a_{1 k}$ in Eq. (8.14) is equivalent to

$$
a_{1 k}=\exp \{\ln (0.5)+k[\ln (20)-\ln (0.5)] / n A 1\}
$$

and is used because of the logtriangular distributions associated with $a_{1}$. Values of $n T=100, n A 1=10$ and $n A 2=$ 10 are used for illustration.

The approximation procedure indicated in Eq. (8.12) with $n T=100, n A 1=10$ and $n A 2=10$ requires evaluation of the functions $D_{D}\left(\tau \mid t_{j}, a_{1 k}, a_{2 l}, \mathbf{e}_{D i}\right)$ for $j=0,1, \ldots, 100, k=0,1, \ldots, 10$, and $l=0,1, \ldots, 10$ (i.e., the evaluation of $(100)(11)(11)=12,100$ functions; a factor of 100 rather than 101 is used in the preceding since the curve $D_{D}(\tau \mid b$, $\left.a_{1 k}, a_{2 l}, \mathbf{e}_{D i}\right)=0$ for $a \leq \tau \leq b$ and thus requires no numerical evaluation) to estimate $E_{A}\left[D_{D}\left(\tau \mid \mathbf{a}, \mathbf{e}_{D i}\right) \mid \mathbf{e}_{A i}\right]$. For perspective, plots of $D_{D}\left(\tau \mid t_{j}, a_{15}, a_{25}, \mathbf{e}_{D i}\right)$ for $j=0,1, \ldots, 99$ are shown in Fig. 4. Thus, Fig. 4 displays 100 of the 12,100 functions used in the estimation of $E_{A}\left[D_{D}\left(\tau \mid \mathbf{a}, \mathbf{e}_{D i}\right) \mid \mathbf{e}_{A i}\right]$.

As an aside, it is important to recognize that formal quadrature-based procedures are only one of a variety of approaches that could be used to approximate the integrals that define $E_{A}\left[D_{D}\left(\tau \mid \mathbf{a}, \mathbf{e}_{D i}\right) \mid \mathbf{e}_{A i}\right]$. In general, the nature of $D_{D}\left(\tau \mid \mathbf{a}, \mathbf{e}_{D}\right)$ must be examined very carefully so that an appropriate and efficient numerical integration procedure can be identified. Specifically, $D_{D}\left(\tau \mid \mathbf{a}, \mathbf{e}_{D}\right)$ may have special properties that can be utilized in developing an efficient numerical integration procedure.

Each element of the LHS indicated in Eq. (8.10) results in a different value for $E_{A}\left[D_{D}\left(\tau \mid \mathbf{a}, \mathbf{e}_{D}\right) \mid \mathbf{e}_{A}\right]$. Specifically, a sequence 


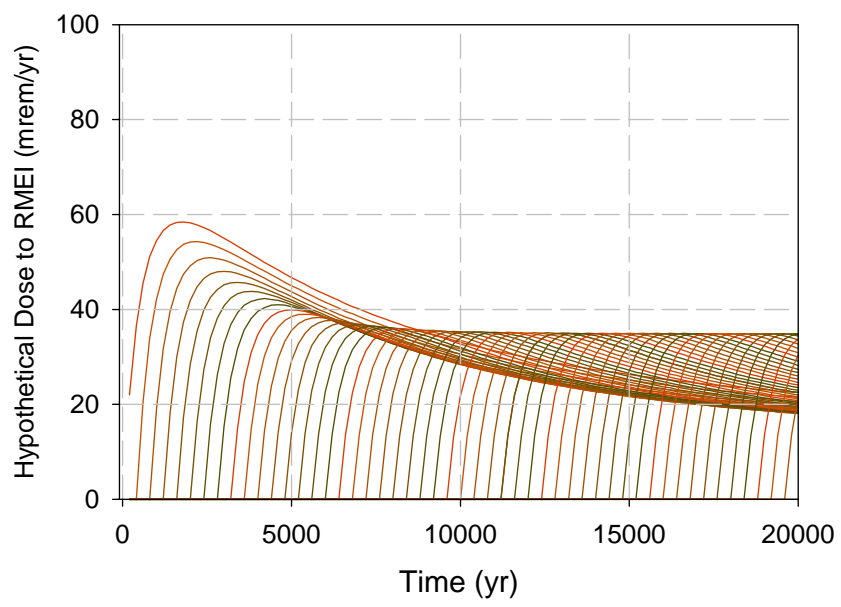

Fig. 4. Hypothetical dose curves $D_{D}\left(\tau \mid t_{j}, a_{15}, a_{25}, \mathbf{e}_{D i}\right), j=0,2,4, \ldots, 98$, used in estimation of $E_{A}\left[D_{D}(\tau \mid \mathbf{a}\right.$, $\left.\left.\mathbf{e}_{D 1}\right) \mid \mathbf{e}_{A 1}\right]$ as described in conjunction with Eq. (8.12).

$$
E_{A}\left[D_{D}\left(\tau \mid \mathbf{a}, \mathbf{e}_{D i}\right) \mid \mathbf{e}_{A i}\right] \cong \hat{E}_{A}\left[D_{D}\left(\tau \mid \mathbf{a}, \mathbf{e}_{D i}\right) \mid \mathbf{e}_{A i}\right]
$$

of $i=1,2, \ldots, n S=100$ estimates for $E_{A}\left[D_{D}\left(\tau \mid \mathbf{a}, \mathbf{e}_{D i}\right) \mid \mathbf{e}_{A i}\right]$ is obtained (Figs. 5a and 5c) with the individual estimates $\hat{E}_{A}\left[D\left(\tau \mid \mathbf{a}, \mathbf{e}_{D i}\right) \mid \mathbf{e}_{A i}\right]$ determined as indicated in Eq. (8.12). The spread of the expected dose curves in Figs. 5a and 5c is providing an indication of the epistemic uncertainty in the expected dose to the RMEI that arises from aleatory uncertainty (i.e., from properties of the disruptive event that are assumed to be random). To facilitate inspection of the individual curves, expected dose is presented on both a linear scale (Fig. 5a) and a log scale (Fig. 5c).

A more formal representation of the uncertainty in the expected dose curves displayed in Figs. $5 \mathrm{a}$ and $5 \mathrm{c}$ can be obtained by presenting the expected curve $E_{E}\left\{E_{A}\left[D_{D}\left(\tau \mid \mathbf{a}, \mathbf{e}_{D}\right) \mid \mathbf{e}_{A}\right]\right\}$ and associated probabilities $p_{E}\left\{E_{A}\left[D_{D}(\tau \mid \mathbf{a}\right.\right.$, $\left.\left.\left.\mathbf{e}_{D}\right) \mid \mathbf{e}_{A}\right] \leq D\right\}$ that derive from epistemic uncertainty. Specifically, these quantities are formally defined in Eqs. (8.1) and (8.2), and approximation procedures that can be used in conjunction with the LHS indicated in Eq. (8.10) are presented in Eqs. (8.8) and (8.9). The resultant approximations for $E_{E}\left\{E_{A}\left[D_{D}\left(\tau \mid \mathbf{a}, \mathbf{e}_{D}\right) \mid \mathbf{e}_{A}\right]\right\}$ and $p_{E}\left\{E_{A}\left[D_{D}(\tau \mid \mathbf{a}\right.\right.$, $\left.\left.\left.\mathbf{e}_{D}\right) \mid \mathbf{e}_{A}\right] \leq D\right\}$ are shown in Figs. 5b and 5d, with both linear (Fig. 5b) and log (Fig. 5d) plots given. Because both $E_{E}\left\{E_{A}\left[D_{D}\left(\tau \mid \mathbf{a}, \mathbf{e}_{D}\right) \mid \mathbf{e}_{A}\right]\right\}$ and $E_{A}\left[D_{D}\left(\tau \mid \mathbf{a}, \mathbf{e}_{D}\right) \mid \mathbf{e}_{A}\right]$ are expected value curves, the approximation to $E_{E}\left\{E_{A}\left[D_{D}(\tau \mid \mathbf{a}\right.\right.$, $\left.\left.\left.\mathbf{e}_{D}\right) \mid \mathbf{e}_{A}\right]\right\}$ in Fig. 5 and in other similar figures is labeled "Expected (Combined Mean)" to indicate that an expected value over both epistemic uncertainty and aleatory uncertainty is being presented; when appropriate, a similar designation is used in the text. The CDF for expected dose defined by $p_{E}\left\{E_{A}\left[D_{D}\left(\tau \mid \mathbf{a}, \mathbf{e}_{D}\right) \mid \mathbf{e}_{A}\right] \leq D\right\}$ at each time $\tau$ is summarized by presenting the $0.05,0.5$ and 0.95 quantiles for $E_{A}\left[D_{D}\left(\tau \mid \mathbf{a}, \mathbf{e}_{D}\right) \mid \mathbf{e}_{A}\right]$ as functions of time.

The expected (combined mean) and quantile curves in Figs. 5b and 5d were estimated with a LHS of size $n S=$ 100. Thus, there is uncertainty in their values that derives from the inherent variability in analysis outcomes that are obtained with a sampling-based procedure. As discussed in conjunction with Eqs. (7.18) - (7.19), replicated sampling in combination with the $t$-distribution can be used to assess the stability of results obtained with Latin hypercube sampling. In particular, the described approach is used with $n R=10$ replicated LHSs of size $n S=100$ to assess the stability of the results presented in Fig. 5 (Fig. 6). The resultant estimates for $E_{E}\left\{E_{A}\left[D_{D}\left(\tau \mid \mathbf{a}, \mathbf{e}_{D}\right) \mid \mathbf{e}_{A}\right]\right\}$ and the associated 0.05 and 0.5 quantiles appear to be quite stable, with the estimates for the 0.95 quantile showing more variability from sample to sample (Fig. 6).

As described in Eq. (7.19), the $t$-distribution can be used to place a confidence interval around an estimate for $E_{E}\left\{E_{A}\left[D_{D}\left(\tau \mid \mathbf{a}, \mathbf{e}_{D}\right) \mid \mathbf{e}_{A}\right]\right\}$ that derives from the $n R=10$ replicates (Fig. 7). If desired, the same procedure can be used to define confidence intervals for quantiles. Whether or not the observed level of sampling variability is acceptable is a judgment call that has to be made in the context of a specific analysis. There is no universal standard for acceptable variability (i.e., error) in a sampling-based analysis. 


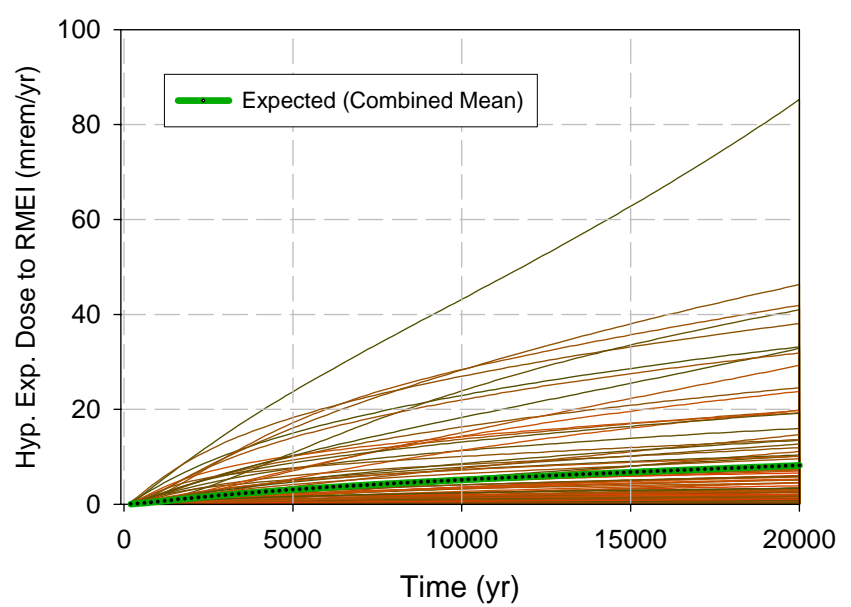

(a)

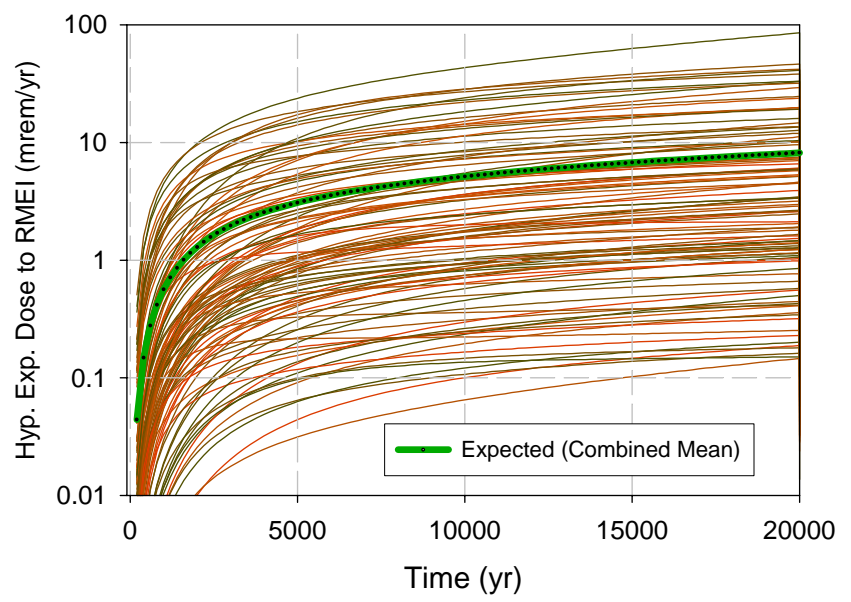

(c)

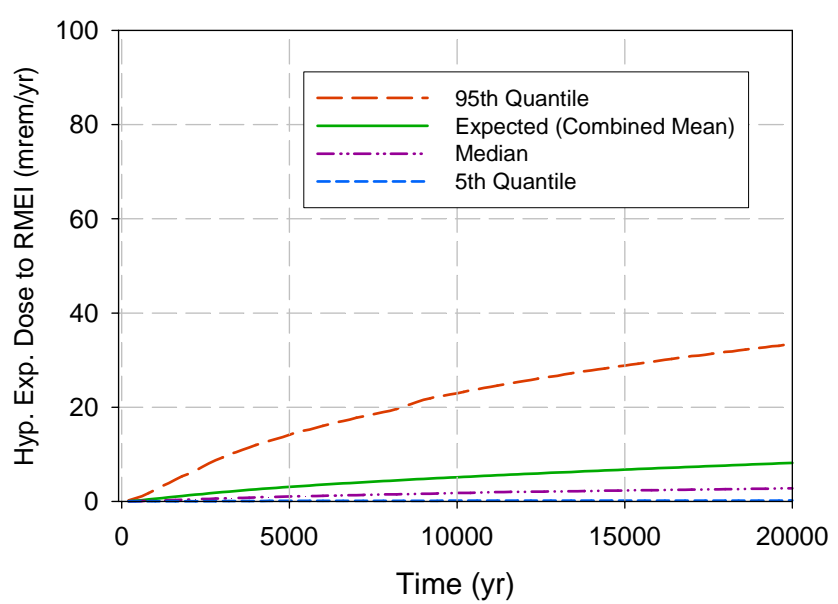

(b)

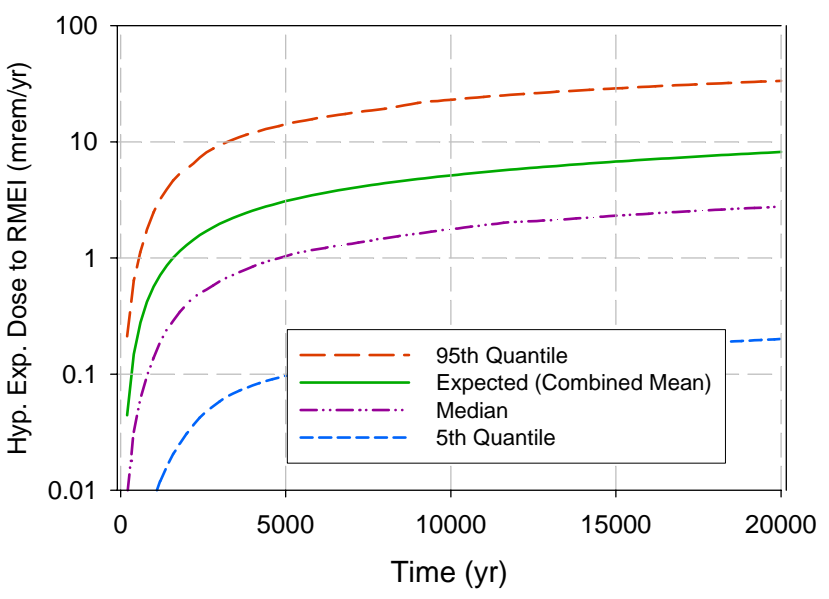

(d)

Fig. 5. Representation of epistemic uncertainty associated with hypothetical expected dose $E_{A}\left[D_{D}\left(\tau \mid \mathbf{a}, \mathbf{e}_{D}\right) \mid \mathbf{e}_{A}\right]$ estimated with Strategy 1 and LHS indicated in Eq. (8.10): (a, b) Expected dose curves $\hat{E}_{A}\left[D_{D}(\tau \mid \mathbf{a}\right.$, $\left.\left.\mathbf{e}_{D i}\right) \mid \mathbf{e}_{A i}\right]$ for $i=1,2, \ldots, n S=100$ with linear and log scales, and (c, d) Expected (combined mean) dose $\hat{E}_{E}\left\{E_{A}\left[D_{D}\left(\tau \mid \mathbf{a}, \mathbf{e}_{D}\right) \mid \mathbf{e}_{A}\right]\right\}$ and associated quantiles (i.e., $\left.0.05,0.5,0.95\right)$ for expected dose $E_{A}\left[D_{D}(\tau \mid \mathbf{a}\right.$, $\left.\left.\mathbf{e}_{D}\right) \mid \mathbf{e}_{A}\right]$ defined by $\hat{p}_{E}\left\{E_{A}\left[D_{D}\left(\tau \mid \mathbf{a}, \mathbf{e}_{D}\right) \mid \mathbf{e}_{A}\right] \leq D\right\}$ with linear and log scales.

It is important to recognize that the quantiles in Fig. 6 and the confidence intervals in Fig. 7 are quantifying two very different types of uncertainty. The quantiles in Fig. 6 derive from epistemic uncertainty with respect to the appropriate values to use for parameters within the analysis, where, in general, the concept of a parameter can be interpreted broadly enough to include alternative models or modeling assumptions. The only way to reduce the epistemic uncertainty in expected dose $E_{A}\left[D_{D}\left(\tau \mid \mathbf{a}, \mathbf{e}_{D}\right) \mid \mathbf{e}_{A}\right]$ quantified by the quantiles in Fig. 6 is to increase knowledge with respect to the system under study and thus reduce the epistemic uncertainty associated with the elements of e. In contrast, the confidence intervals in Fig. 7 derive from sampling variability and, in concept, can be made arbitrarily small by suitably increasing the sample size in use. Specifically, the confidence intervals in Fig. 7 apply to errors in the numerical estimation of $E_{E}\left\{E_{A}\left[D_{D}\left(\tau \mid \mathbf{a}, \mathbf{e}_{D}\right) \mid \mathbf{e}_{A}\right]\right\}$; however, once the entities in the integral in Eq. (8.1) that defines $E_{E}\left\{E_{A}\left[D_{D}\left(\tau \mid \mathbf{a}, \mathbf{e}_{D}\right) \mid \mathbf{e}_{A}\right]\right\}$ are specified, there is no uncertainty in either an epistemic or aleatory sense in $E_{E}\left\{E_{A}\left[D_{D}\left(\tau \mid \mathbf{a}, \mathbf{e}_{D}\right) \mid \mathbf{e}_{A}\right]\right\}$. 


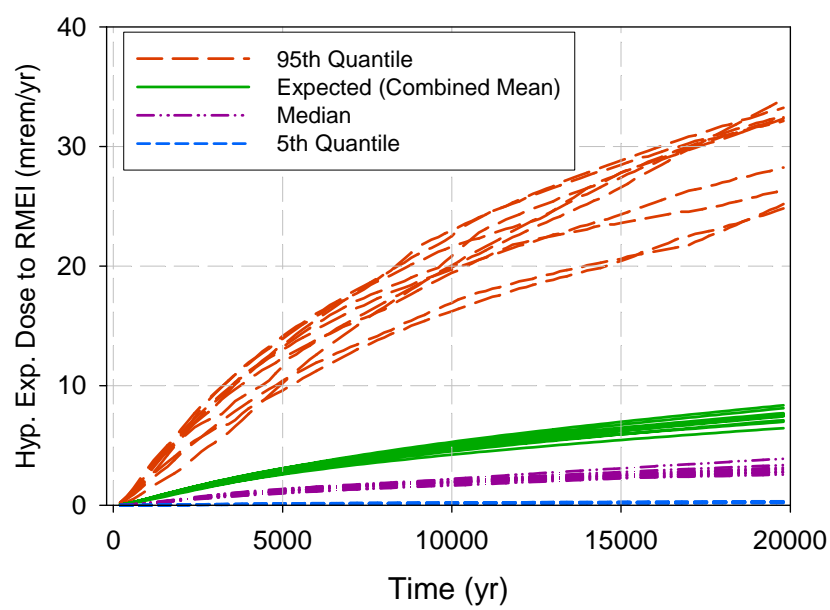

(a)

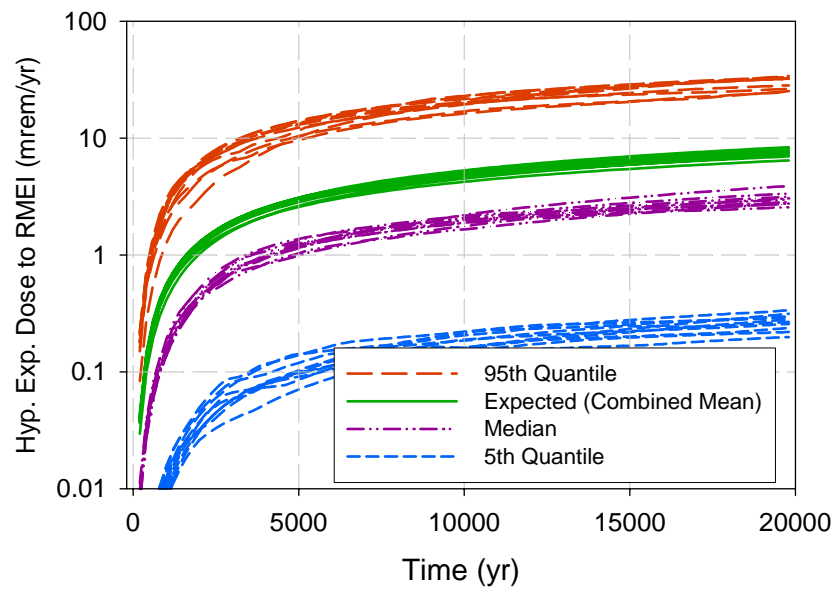

(b)

Fig. 6. Representation of sampling variability in Strategy 1 that results from using $n R=10$ replicated LHSs of size $n S=100$ in the hypothetical estimation of expected (combined mean) dose $E_{E}\left\{E_{A}\left[D_{D}\left(\tau \mid \mathbf{a}, \mathbf{e}_{D}\right) \mid \mathbf{e}_{A}\right]\right\}$ and associated quantiles (i.e., $0.05,0.5,0.95)$ for expected dose $E_{A}\left[D_{D}\left(\tau \mid \mathbf{a}, \mathbf{e}_{D}\right) \mid \mathbf{e}_{A}\right]$ that derive from estimates for $p_{E}\left\{E_{A}\left[D_{D}\left(\tau \mid \mathbf{a}, \mathbf{e}_{D}\right) \mid \mathbf{e}_{A}\right] \leq D\right\}$ : (a) Linear scale, and (b) Log scale.

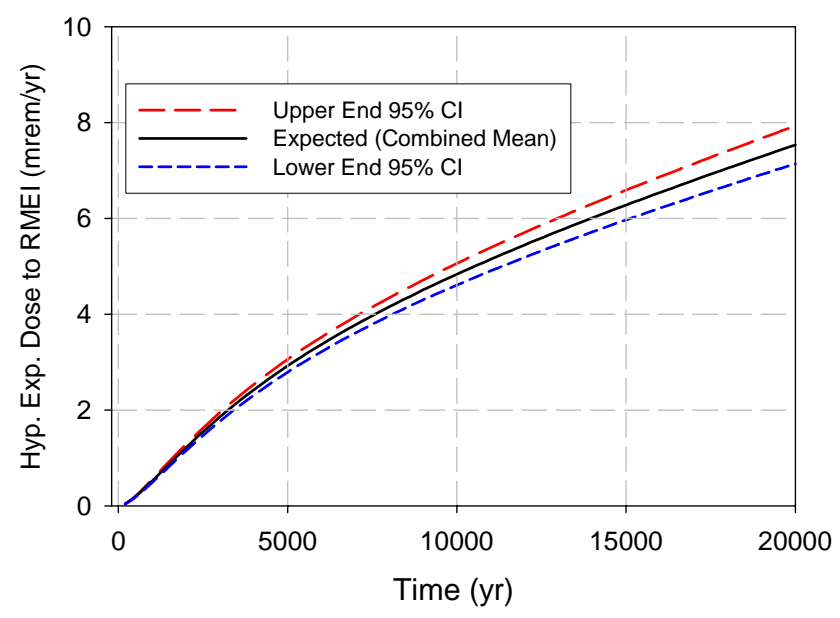

Fig. 7. Hypothetical estimate obtained with Strategy 1 for expected (combined mean) dose $E_{E}\left\{E_{A}\left[D_{D}(\tau \mid \mathbf{a}\right.\right.$, $\left.\left.\left.\mathbf{e}_{D}\right) \mid \mathbf{e}_{A}\right]\right\}$ that derives from $n R=10$ replicated LHSs of size $n S=100$ and associated $95 \%$ confidence intervals (CIs) obtained with the $t$-distribution as indicated in conjunction with Eq. (7.19).

As discussed at the end of Sect. 7, the NRC has specified the following acceptance criteria: "A sufficient number of realizations has been obtained ... to ensure that the results of the calculation are numerically stable" (see Quote (YMRP4)) and "the annual dose curve includes confidence intervals (e.g., 95 $5^{\text {th }}$ and $5^{\text {th }}$ percentile) to represent the uncertainty in the dose calculations" (see Quote (YMRP5)). The criterion in Quote (YMRP4) clearly applies to the numerical stability of a sampling-based calculation, which is what is being characterized by the confidence intervals in Fig. 7. Specifically, the confidence intervals in Fig. 7 provide a representation of the uncertainty in an estimate for $E_{E}\left\{E_{A}\left[D_{D}\left(\tau \mid \mathbf{a}, \mathbf{e}_{D}\right) \mid \mathbf{e}_{A}\right]\right\}$ obtained with a sampling-based procedure. In contrast, the criterion in Quote (YMRP5) can be interpreted as applying to the epistemic uncertainty associated with estimates for the expected dose $E_{A}\left[D_{D}\left(\tau \mid \mathbf{a}, \mathbf{e}_{D}\right) \mid \mathbf{e}_{A}\right]$. With this interpretation, the percentile (i.e., quantile) curves relevant to the criterion in Quote (YMRP5) are the curves in Figs. 5b, 5d, and 6. Specifically, these quantile curves provide a representation of the uncertainty in $E_{A}\left[D_{D}\left(\tau \mid \mathbf{a}, \mathbf{e}_{D}\right) \mid \mathbf{e}_{A}\right]$ that derives from epistemic uncertainty in $\mathbf{e}=\left[\mathbf{e}_{A}, \mathbf{e}_{D}\right]$. If the criterion in Quote (YMRP5) is interpreted as applying to sampling-based error as quantified by results of the form 
shown in Fig. 7, then, in effect, the implications of epistemic uncertainty in the calculation of expected dose are being averaged out of the analysis.

\subsection{Strategy 2: Sample from $\mathcal{E} \times \mathscr{P}$ and Numerically Evaluate Integral over Time}

This strategy is based on rewriting the representation for $E_{E}\left\{E_{A}\left[D_{D}\left(\tau \mid \mathbf{a}, \mathbf{e}_{D}\right) \mid \mathbf{e}_{A}\right]\right\}$ in Eq. (8.1) by changing the order of integration involving $\mathbf{e}, t$ and $\mathbf{p}$. Specifically, $E_{E}\left\{E_{A}\left[D_{D}\left(\tau \mid \mathbf{a}, \mathbf{e}_{D}\right) \mid \mathbf{e}_{A}\right]\right\}$ can be rewritten as

$$
\begin{aligned}
E_{E}\left\{E_{A}\left[D_{D}\left(\tau \mid \mathbf{a}, \mathbf{e}_{D}\right) \mid \mathbf{e}_{A}\right]\right\} & =\int_{\mathcal{E}} \int_{\mathcal{R}}\left[\int_{a}^{\tau} D_{D}\left(\tau \mid t, \mathbf{p}, \mathbf{e}_{D}\right) \lambda_{D} \mathrm{~d} t\right] d_{P}\left(\mathbf{p} \mid \mathbf{e}_{A}\right) d_{E}(\mathbf{e}) \mathrm{d} P \mathrm{~d} E \\
& =\int_{\mathcal{E}} \int_{\mathcal{P}} E_{t}\left[D_{D}\left(\tau \mid \mathbf{p}, \mathbf{e}_{D}, \lambda_{D}\right)\right] d_{P}\left(\mathbf{p} \mid \mathbf{e}_{A}\right) d_{E}(\mathbf{e}) \mathrm{d} P \mathrm{~d} E
\end{aligned}
$$

where

$$
\begin{aligned}
E_{t}\left[D_{D}\left(\tau \mid \mathbf{p}, \mathbf{e}_{D}, \lambda_{D}\right)\right] & =\int_{a}^{\tau} D_{D}\left(\tau \mid t, \mathbf{p}, \mathbf{e}_{D}\right) \lambda_{D} \mathrm{~d} t \\
& =\int_{a}^{b} D_{D}\left(\tau \mid t, \mathbf{p}, \mathbf{e}_{D}\right) \lambda_{D} \mathrm{~d} t
\end{aligned}
$$

is introduced for later notational convenience and is the expected dose at time $\tau$ conditional on $\mathbf{p}, \mathbf{e}_{D}$ and $\lambda_{D}$.

Given the preceding representation, $E_{E}\left\{E_{A}\left[D_{D}\left(\tau \mid \mathbf{a}, \mathbf{e}_{D}\right) \mid \mathbf{e}_{A}\right]\right\}$ can be approximated by sampling from $\mathcal{E} \times \mathscr{P}$ and numerically integrating over time. Specifically,

$$
\hat{E}_{E}\left\{E_{A}\left[\left(\tau \mid \mathbf{a}, \mathbf{e}_{D}\right) \mid \mathbf{e}_{A}\right]\right\}=\sum_{i=1}^{n S} E_{t}\left[D_{D}\left(\tau \mid \mathbf{p}_{i}, \mathbf{e}_{D i}, \lambda_{D i}\right)\right] / n S,
$$

where

$$
\left[\mathbf{e}_{i}, \mathbf{p}_{i}\right]=\left[\mathbf{e}_{A i}, \mathbf{e}_{D i}, \mathbf{p}_{i}\right], i=1,2, \ldots, n S,
$$

is a sample generated from $\mathcal{E} \times \mathscr{P}$ in consistency with the distributions assigned to the individual elements of $\mathbf{e}_{A}, \mathbf{e}_{D}$ and $\mathbf{p}$. As a reminder, $\lambda_{D}$ is assumed to be an element of $\mathbf{e}_{A}$. If an element $a$ of $\mathbf{p}$ has a distribution that depends on one or more elements of $\mathbf{e}_{A}$, then these elements must be sampled and used to define the distribution for $a$ for sample element $i$ before the corresponding value $a_{i}$ for $a$ can be sampled. In turn, $E_{t}\left[D_{D}\left(\tau \mid \mathbf{p}_{i}, \mathbf{e}_{D i}, \lambda_{D i}\right)\right]$ can be approximated by

$$
\hat{E}_{t}\left[D_{D}\left(\tau \mid \mathbf{p}_{i}, \mathbf{e}_{D i}, \lambda_{D i}\right)\right]=\lambda_{D i} \sum_{j=1}^{n T}\left\{\left[D_{D}\left(\tau \mid t_{j-1}, \mathbf{p}_{i}, \mathbf{e}_{D i}\right)+D_{D}\left(\tau \mid t_{j}, \mathbf{p}_{i}, \mathbf{e}_{D i}\right)\right] / 2\right\} \Delta t_{j},
$$

where $t_{j}$ and $\Delta t_{j}$ are defined as indicated in Eq. (8.13). The approximation

$$
\hat{E}\left\{E_{A}\left[D\left(\tau \mid \mathbf{a}, \mathbf{e}_{D}\right) \mid \mathbf{e}_{A}\right]\right\}=\sum_{i=1}^{n S}\left(\lambda_{D i} \sum_{j=1}^{n T}\left\{\left[D_{D}\left(\tau \mid t_{j-1}, \mathbf{p}_{i}, \mathbf{e}_{D i}\right)+D_{D}\left(\tau \mid t_{j}, \mathbf{p}_{i}, \mathbf{e}_{D i}\right)\right] / 2\right\} \Delta t_{j}\right) / n S
$$

to $E\left\{E_{A}\left[D\left(\tau \mid \mathbf{a}, \mathbf{e}_{D}\right) \mid \mathbf{e}_{A}\right]\right\}$ results by combining the approximations in Eqs. (8.21) and (8.23).

The implementation of Strategy 2 is now illustrated with the function $D_{D}\left(\tau \mid t, \mathbf{p}, \mathbf{e}_{D}\right)$ and associated definitions for $\mathbf{p}, \mathbf{e}_{A}$ and $\mathbf{e}_{D}$ introduced in conjunction with Eq. (8.3). This illustration initially uses a LHS 


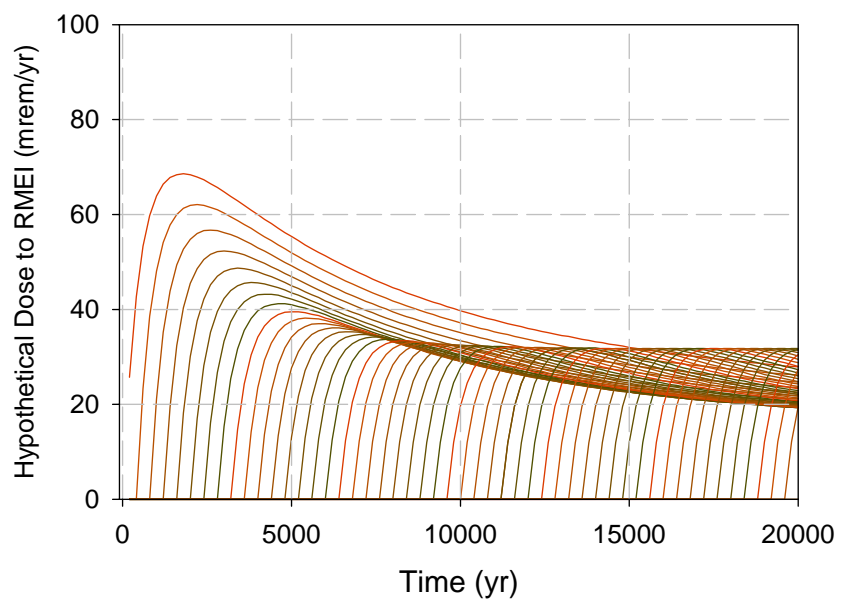

Fig. 8. Hypothetical dose curves $D_{D}\left(\tau \mid t_{j}, a_{11}, a_{21}, \mathbf{e}_{D 1}\right), j=0,2,4, \ldots, 98$, for sample element $\mathbf{e}_{1}$ of sample in Eq. (8.26) used in estimation of conditional expected dose $E_{t}\left[D_{D}\left(\tau \mid \mathbf{p}_{1}, \mathbf{e}_{D 1}, \lambda_{D 1}\right)\right]$ with Strategy 2 as described in conjunction with Eq. (8.23).

$$
\begin{aligned}
\tilde{\mathbf{e}}_{i} & =\left[\mathbf{e}_{A i}, \mathbf{e}_{D i}, \mathbf{r}_{i}\right] \\
& =\left[e_{A 1 i}, e_{A 2 i}, e_{A 3 i}, e_{D 1 i}, e_{D 2 i}, \ldots, e_{D 9 i}, r_{1 i}, r_{2 i}\right]
\end{aligned}
$$

of size $n S=100$ from the set $\mathcal{E} \times \mathbb{R}$, where $\mathcal{E}$ is the set of possible values for $\mathbf{e}=\left[\mathbf{e}_{A}, \mathbf{e}_{D}\right]$ and $\mathbb{R}=[0,1] \times[0,1]$ is the set of possible values for $\mathbf{r}=\left[r_{1}, r_{2}\right]$. The preceding LHS is generated in consistency with the distributions that characterize the epistemic uncertainty in the elements of $\mathbf{e}$ specified in Tables 2 and 3 and uniform distributions for the two elements of $\mathbf{r}$ on [0,1]. In turn, once the sample in Eq. (8.25) is generated, $r_{1 i}$ and $r_{2 i}$ are used to select values $a_{1 i}$ and $a_{2 i}$ from the distributions for $a_{1}$ and $a_{2}$ defined by $e_{A 1 i}$ and $e_{A 2 i}$. The result is the sample

$$
\begin{aligned}
\mathbf{e}_{i} & =\left[\mathbf{e}_{A i}, \mathbf{e}_{D i}, \mathbf{p}_{i}\right] \\
& =\left[e_{A 1 i}, e_{A 2 i}, e_{A 3 i}, e_{D 1 i}, e_{D 2 i}, \ldots, e_{D 9 i}, a_{1 i}, a_{2 i}\right]
\end{aligned}
$$

of size $n S=100$ from $\mathcal{E} \times \mathscr{P}$.

The outcome of evaluating $D_{D}\left(\tau \mid t_{j}, \mathbf{p}_{1}, \mathbf{e}_{D 1}\right)$ for $j=0,1, \ldots, 99$ is illustrated in Fig. 8. In turn, the 100 approximations to $D_{D}\left(\tau \mid t_{j}, \mathbf{p}_{1}, \mathbf{e}_{D 1}\right)$ in Fig. 8 are used to estimate $E_{t}\left[D_{D}\left(\tau \mid \mathbf{p}_{1}, \mathbf{e}_{D 1}, \lambda_{D 1}\right)\right]$ as indicated in Eq. (8.23). The 100 approximations to $E_{t}\left[D_{D}\left(\tau \mid \mathbf{p}, \mathbf{e}_{D}, \lambda_{D}\right)\right]$ that result for the sample in Eq. (8.26) are shown in Fig. 9. Specifically, the curves in Fig. 9 are plots of $E_{t}\left[D_{D}\left(\tau \mid \mathbf{p}_{i}, \mathbf{e}_{D i}, \lambda_{D i}\right)\right]$ for $i=1,2, \ldots, 100$. Further, the 100 values for $\hat{E}_{t}\left[D_{D}\left(\tau \mid \mathbf{p}_{i}, \mathbf{e}_{D i}, \lambda_{D i}\right)\right]$ result in the approximation $\hat{E}_{E}\left\{E_{A}\left[D_{D}\left(\tau \mid \mathbf{a}, \mathbf{e}_{D}\right) \mid \mathbf{e}_{A}\right]\right\}$ to $E_{E}\left\{E_{A}\left[D_{D}\left(\tau \mid \mathbf{a}, \mathbf{e}_{D}\right) \mid \mathbf{e}_{A}\right]\right\}$ that is also shown in Fig. 9, with this approximation obtained as indicated in Eq. (8.24).

As presented in this example, Strategy 2 for the estimation of $E_{E}\left\{E_{A}\left[D_{D}\left(\tau \mid \mathbf{a}, \mathbf{e}_{D}\right) \mid \mathbf{e}_{A}\right]\right\}$ is less demanding computationally than Strategy 1. In particular, 100 evaluations of $D_{D}\left(\tau \mid t, a_{1}, a_{2}, \mathbf{e}_{D}\right)$ of the form shown in Fig. 8 are used in the determination of each curve $\hat{E}_{t}\left[D_{D}\left(\tau \mid \mathbf{p}, \mathbf{e}_{D}, \lambda_{D}\right)\right]$ in Fig. 9 . In contrast, 12,100 evaluations of $D_{D}\left(\tau \mid t, a_{1}\right.$, $\left.a_{2}, \mathbf{e}_{D}\right)$ are used in the evaluation of each curve $\hat{E}_{A}\left[D_{D}\left(\tau \mid \mathbf{a}, \mathbf{e}_{D}\right) \mid \mathbf{e}_{A}\right]$ in Figs. $5 \mathrm{a}$ and 5c. Thus, while Fig. 8 shows all evaluations of $D_{D}\left(\tau \mid t_{j}, a_{11}, a_{21}, \mathbf{e}_{D 1}\right)$ that result for $j=0,1, \ldots, 99$ and are used in the determination of $\hat{E}_{t}\left[D_{D}\left(\tau \mid \mathbf{p}_{1}, \mathbf{e}_{D 1}, \lambda_{D 1}\right)\right]$, Fig. 4 only shows 100 out of the 12,100 evaluations of $D_{D}\left(\tau \mid t_{j}, a_{1 k}, a_{2 l}, \mathbf{e}_{D 1}\right)$ that result for $j=0,1, \ldots, 99, k=0,1, \ldots, 10$ and $l=0,1, \ldots, 10$, and are used in the determination of $\hat{E}_{A}\left[D_{D}\left(\tau \mid \mathbf{a}, \mathbf{e}_{D 1}\right) \mid \mathbf{e}_{\mathrm{A} 1}\right]$. However, it is important to recognize that no attempt has been made in this example to minimize the required number of evaluations of $D_{D}\left(\tau \mid t, \mathbf{p}, \mathbf{e}_{D}\right)$ in the numerical implementations of Strategies 1 and 2. In a real analysis, the evaluation of $D_{D}\left(\tau \mid t, \mathbf{p}, \mathbf{e}_{D}\right)$ would be a major computational cost, and the properties of $D_{D}\left(\tau \mid t, \mathbf{p}, \mathbf{e}_{D}\right)$ would be very carefully studied to find ways to minimize this cost. 


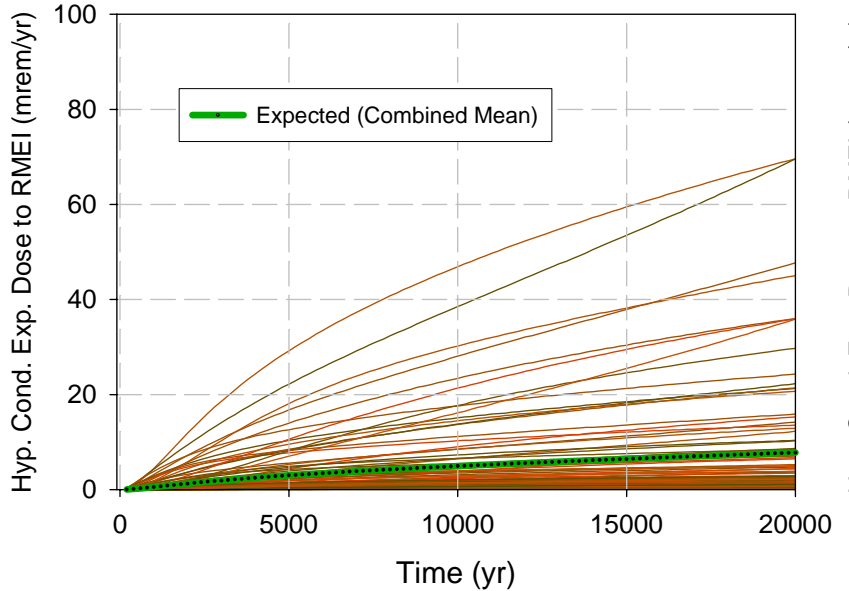

(a)

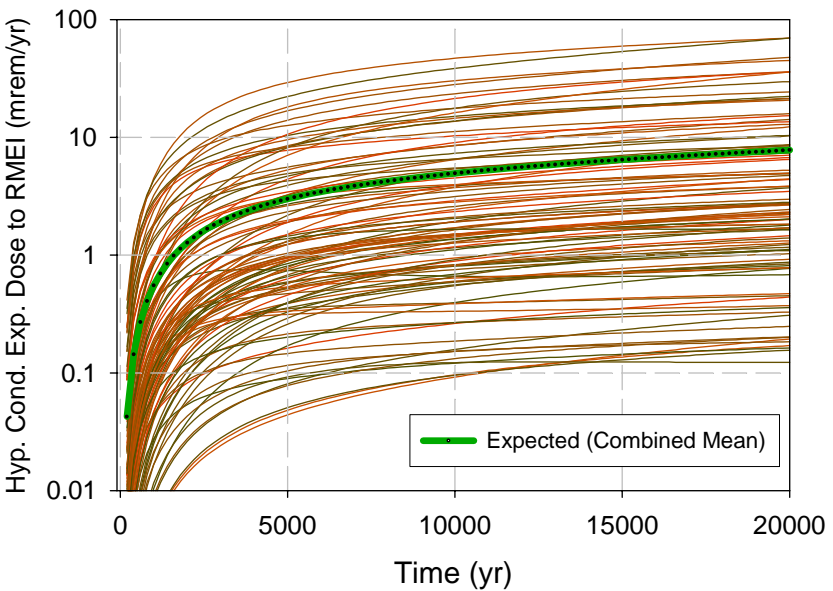

(b)

Fig. 9. Hypothetical estimates obtained with Strategy 2 for conditional expected dose $E_{t}\left[D_{D}\left(\tau \mid \mathbf{p}_{i}, \mathbf{e}_{D i}, \lambda_{D i}\right)\right], i=1$, $2, \ldots, n S=100$, and expected (combined mean) dose $E_{E}\left\{E_{A}\left[D_{D}\left(\tau \mid \mathbf{a}, \mathbf{e}_{D}\right) \mid \mathbf{e}_{A}\right]\right\}$ obtained as described in Eqs. (8.23) and (8.24) for the sample in Eq. (8.26): (a) Linear scale, and (b) Log scale.

Although the distributions of the curves in Figs. 5a and 5c and in Figs. 9a and 9b appear similar on a superficial level, it is important to recognize that these distributions involve very different entities. In particular, the ensemble of curves in Figs. 5a and 5c is displaying the uncertainty in the expected dose $E_{A}\left[D_{D}\left(\tau \mid \mathbf{a}, \mathbf{e}_{D}\right) \mid \mathbf{e}_{A}\right]$ that derives from epistemic uncertainty with respect to the appropriate values to use for the elements of $\mathbf{e}$. Thus, the spread of the curves in Figs. 5a and 5c is providing information on the uncertainty in the expected dose that can be meaningfully summarized by CDFs as indicated in Eqs. (8.2) and (8.9) and displayed as quantile curves in Figs. 5b and 5d. In contrast, the ensemble of curves in Figs. $9 \mathrm{a}$ and $9 \mathrm{~b}$ is displaying intermediate results (i.e., $\hat{E}_{t}\left[D_{D}\left(\tau \mid \mathbf{p}_{i}, \mathbf{e}_{D i}\right.\right.$, $\left.\left.\lambda_{D i}\right)\right]$ as defined in Eq. (8.23)) used in the approximation to $E_{E}\left\{E_{A}\left[D_{D}\left(\tau \mid \mathbf{a}, \mathbf{e}_{D}\right) \mid \mathbf{e}_{A}\right]\right\}$ defined in Eq. (8.24). Although the curves $\hat{E}_{t}\left[D_{D}\left(\tau \mid \mathbf{p}_{i}, \mathbf{e}_{D i}, \lambda_{D i}\right)\right]$ correspond to valid intermediate results used in the approximation of $E_{E}\left\{E_{A}\left[D_{D}(\tau \mid \mathbf{a}\right.\right.$, $\left.\left.\left.\mathbf{e}_{D}\right) \mid \mathbf{e}_{A}\right]\right\}$, it is difficult to give a useful epistemic uncertainty interpretation to the spread of the curves in Fig. 9 because the values for the aleatory variables $a_{1}$ and $a_{2}$ are fixed in the determination of each dose curve $\hat{E}_{t}\left[D_{D}\left(\tau \mid \mathbf{p}_{i}\right.\right.$, $\left.\mathbf{e}_{D i}, \lambda_{D i}\right)$ ] (e.g., this is like assuming all seismic events have the same peak ground velocity or that all igneous intrusions damage the same number of waste packages). The similar appearance of the curves in Figs. 5a, 5c, 9a and 9b is a property of the particular example that was selected for use; different examples could result in the distributions of curves in these figures having very different appearances.

As for Strategy 1, replicated sampling in combination with the $t$-distribution can be used to assess the stability of estimates for $E_{E}\left\{E_{A}\left[D_{D}\left(\tau \mid \mathbf{a}, \mathbf{e}_{D}\right) \mid \mathbf{e}_{A}\right]\right\}$ obtained with Strategy 2 (Fig. 10). Thus, compliance with the acceptance criterion in Quote (YMRP4) can be determined. However, estimates of the epistemic uncertainty associated with expected dose over aleatory uncertainty (i.e., $\left.E_{A}\left[D_{D}\left(\tau \mid \mathbf{a}, \mathbf{e}_{D}\right) \mid \mathbf{e}_{A}\right]\right)$ are not possible. Thus, with Strategy 2, results associated with only one of the two possible interpretations of the acceptance criterion in Quote (YMRP5) discussed at the end of Sect. 8.1 are obtainable. In particular, quantile curves of the form shown in Figs. 5b, 5d and 6 representing the epistemic uncertainty in $E_{A}\left[D_{D}\left(\tau \mid \mathbf{a}, \mathbf{e}_{D}\right) \mid \mathbf{e}_{A}\right]$ are not obtainable with Strategy 2.

\subsection{Strategy 3: Sample from $\mathcal{E} \times \mathscr{P} \times[a, b]$ with Importance Sampling on Time}

This strategy is based on using a sampling-based approach to simultaneously evaluate the three iterated integrals that define $E_{E}\left\{E_{A}\left[D_{D}\left(\tau \mid \mathbf{a}, \mathbf{e}_{D}\right) \mid \mathbf{e}_{A}\right]\right\}$ in Eq. (8.1). To do this, the integral involving time must be rewritten with a density function defined for time. This rewriting produces 


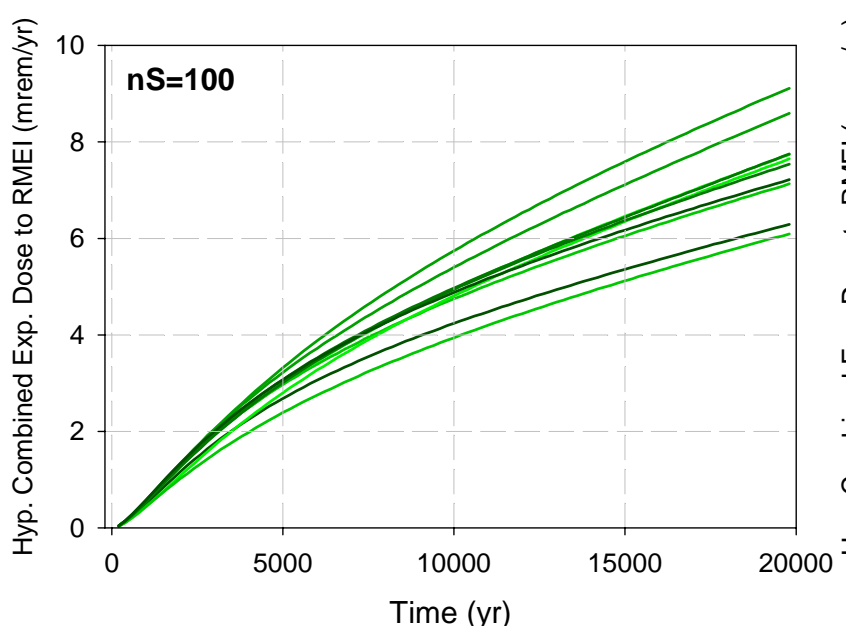

(a)

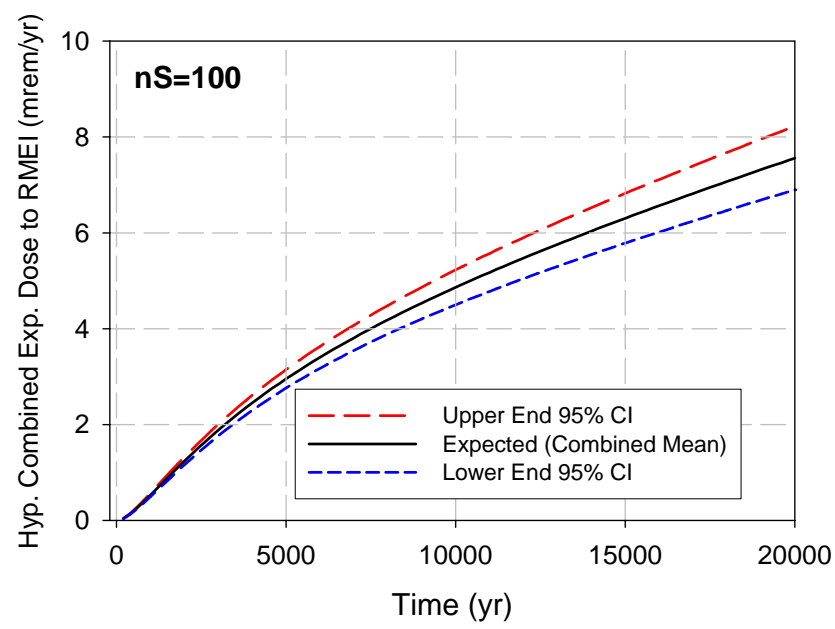

(c)

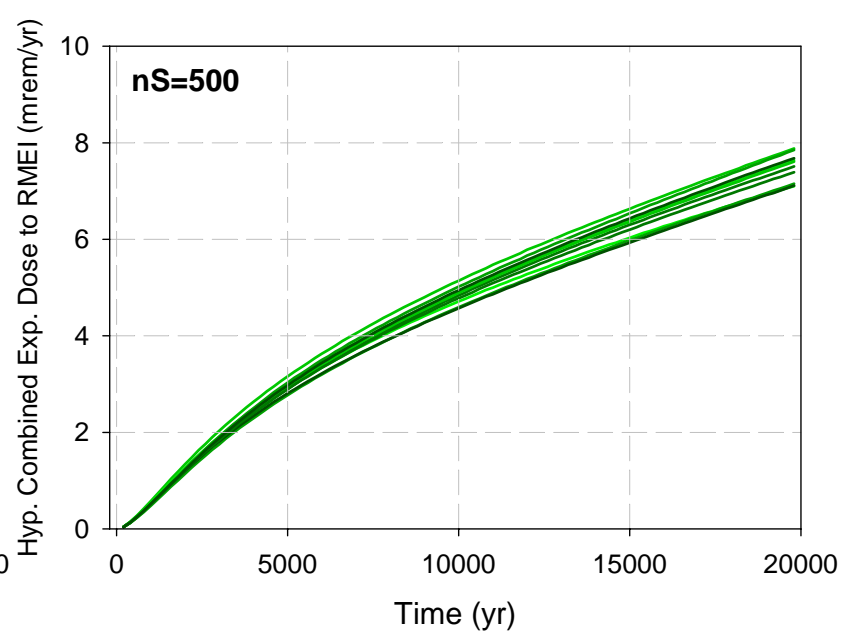

(b)

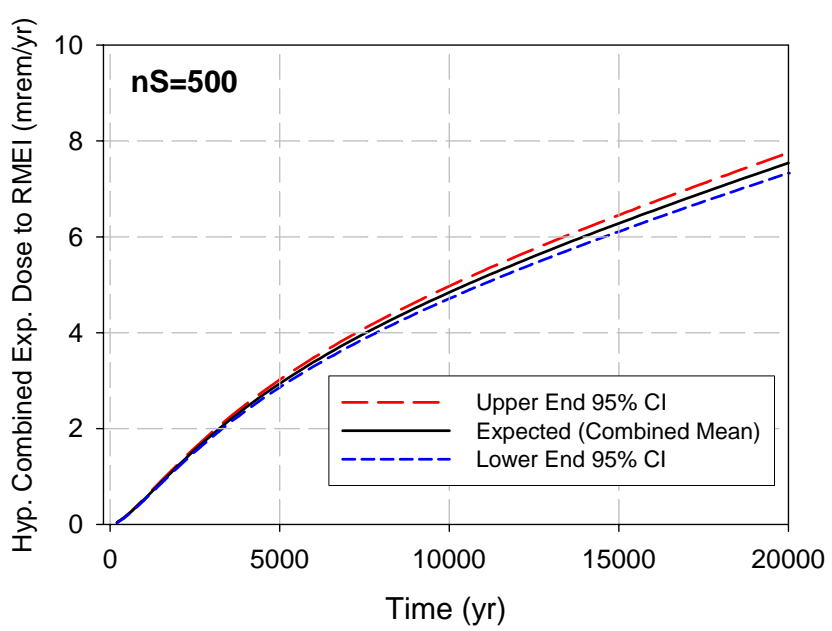

(d)

Fig. 10. Representation of sampling variability that results from using $n R=10$ replicated LHSs of size $n S=100$ and $n S=500$ in the hypothetical estimation of expected (combined mean) dose $E_{E}\left\{E_{A}\left[D_{D}\left(\tau \mid \mathbf{a}, \mathbf{e}_{D}\right) \mid \mathbf{e}_{A}\right]\right\}$ with Strategy 2: (a, b) Replicated estimates $\hat{E}_{E r}\left\{E_{A}\left[D_{D}\left(\tau \mid \mathbf{a}, \mathbf{e}_{D}\right) \mid \mathbf{e}_{A}\right]\right\}, r=1,2, \ldots, 10$, and (c, d) Estimates for $E_{E}\left\{E_{A}\left[D_{D}\left(\tau \mid \mathbf{a}, \mathbf{e}_{D}\right) \mid \mathbf{e}_{A}\right]\right\}$ and associated $95 \%$ confidence intervals (CIs) that derive from the replicated estimates $\hat{E}_{E r}\left\{E_{A}\left[D_{D}\left(\tau \mid \mathbf{a}, \mathbf{e}_{D}\right) \mid \mathbf{e}_{A}\right]\right\}$.

$$
E_{E}\left\{E_{A}\left[D_{D}\left(\tau \mid \mathbf{a}, \mathbf{e}_{D}\right) \mid \mathbf{e}_{A}\right]\right\}=\int_{\mathcal{E}} \int_{\mathcal{P}} \int_{a}^{b}\left[\frac{D_{D}\left(\tau \mid t, \mathbf{p}, \mathbf{e}_{D}\right) \lambda_{D}}{d_{t}(t)}\right] d_{t}(t) d_{P}\left(\mathbf{p} \mid \mathbf{e}_{A}\right) d_{E}(\mathbf{e}) \mathrm{d} t \mathrm{~d} P \mathrm{~d} E,
$$

where $d_{t}(t)$ is the introduced density function for time and the integral over time can be written from $a$ to $b$ rather than from $a$ to $\tau$ because $D_{D}\left(\tau \mid t, \mathbf{p}, \mathbf{e}_{D}\right)=0$ for $\tau<t$.

The iterated integrals in Eq. (8.27) are defining the expected value of the function

$$
f\left(\tau \mid t, \mathbf{p}, \mathbf{e}_{D}\right)=D_{D}\left(\tau \mid t, \mathbf{p}, \mathbf{e}_{D}\right) \lambda_{D} / d_{t}(t)
$$

with respect to the density functions $d_{t}(t), d_{P}\left(\mathbf{p} \mid \mathbf{e}_{A}\right)$ and $d_{E}(\mathbf{e})$ defined on $[a, b], \mathscr{P}$ and $\mathcal{E}$, respectively. The expected value for $f\left(\tau \mid t, \mathbf{p}, \mathbf{e}_{D}\right)$, and hence the value for $E_{E}\left\{E_{A}\left[D_{D}\left(\tau \mid \mathbf{a}, \mathbf{e}_{D}\right) \mid \mathbf{e}_{A}\right]\right\}$, can be obtained by sampling from 
$[a, b], \mathscr{P}$ and $\mathcal{E}$ in consistency with the density functions $d_{t}(t), d_{P}\left(\mathbf{p} \mid \mathbf{e}_{A}\right)$ and $d_{E}(\mathbf{e})$. Specifically, an estimate for $E_{E}\left\{E_{A}\left[D_{D}\left(\tau \mid \mathbf{a}, \mathbf{e}_{D}\right) \mid \mathbf{e}_{A}\right]\right\}$ is given by

$$
\hat{E}_{E}\left\{E_{A}\left[D\left(\tau \mid \mathbf{a}, \mathbf{e}_{D}\right) \mid \mathbf{e}_{A}\right]\right\}=\sum_{i=1}^{n S}\left[\frac{D_{D}\left(\tau \mid t_{i}, \mathbf{p}_{i}, \mathbf{e}_{D i}\right) \lambda_{D i}}{d_{t}\left(t_{i}\right)}\right] / n S,
$$

where

$$
\mathbf{s}_{i}=\left[\mathbf{e}_{i}, \mathbf{p}_{i}, t_{i}\right]=\left[\mathbf{e}_{A i}, \mathbf{e}_{D i}, \mathbf{p}_{i}, t_{i}\right], i=1,2, \ldots, n S,
$$

is a random or LHS from $\mathcal{E} \times \mathscr{P} \times[a, b]$ generated in consistency with the density functions $d_{E}(\mathbf{e}), d_{P}\left(\mathbf{p} \mid \mathbf{e}_{A}\right)$ and $d_{t}(t)$.

The density function $d_{t}(t)$ is determining the relative concentrations of time values over the interval $[a, b]$. The use of such a density function in the evaluation of an integral is often referred to as importance sampling ${ }^{173-179}$ because, in concept, the density function can be chosen to place heavy concentrations of sampled values in regions that are believed to be important to the value of the integral under consideration and fewer points in regions that are believed to be less important to the value of the integral. Two possible definitions for $d_{t}(t)$ are

$$
d_{t, u}(t)=1 /(b-a)
$$

and

$$
d_{t, l n}(t)=1 /[t \ln (b / a)]
$$

with the first definition corresponding to a uniform distribution on $[a, b]$ and the second definition corresponding to a loguniform distribution on $[a, b]$. Thus, the definition of $d_{t, u}(t)$ in Eq. (8.31) corresponding to a uniform distribution does not emphasize any particular subrange of $[a, b]$; in contrast, the definition of $d_{t, l n}(t)$ in Eq. (8.32) corresponding to a loguniform distribution emphasizes values of $t$ close to $a$. Use of the loguniform distribution requires $a>0$ (e.g., $[10,10,000 \mathrm{yr}]$ rather than $[0,10,000 \mathrm{yr}]$ ). In general, $d_{t}(t)$ could be any density function defined on $[a$, $b]$ as long as $d_{t}(t) \neq 0$ for $a \leq t \leq b$.

Use of different definitions for $d_{t}(t)$ results in different appearing approximations to $E_{E}\left\{E_{A}\left[D_{D}(\tau \mid \mathbf{a}) \mid \mathbf{e}\right]\right\}$. For example, the approximation in Eq. (8.29) becomes

$$
\hat{E}_{E}\left\{E_{A}\left[D_{D}\left(\tau \mid \mathbf{a}, \mathbf{e}_{D}\right) \mid \mathbf{e}_{A}\right]\right\}=(b-a) \sum_{i=1}^{n S} D_{D}\left(\tau \mid t_{i}, \mathbf{p}_{i}, \mathbf{e}_{D i}\right) \lambda_{D i} / n S
$$

for uniform sampling on $[a, b]$ with the density function $d_{t, u}(t)$ in Eq. (8.31) and

$$
\hat{E}_{E}\left\{E_{A}\left[D_{D}\left(\tau \mid \mathbf{a}, \mathbf{e}_{D}\right) \mid \mathbf{e}_{A}\right]\right\}=\ln (b / a) \sum_{i=1}^{n S} t_{i} D_{D}\left(\tau \mid t_{i}, \mathbf{p}_{i}, \mathbf{e}_{D i}\right) \lambda_{D i} / n S
$$

for loguniform sampling on $[a, b]$ with the density function $d_{t, l n}(t)$ in Eq. (8.32). The choice of the density function $d_{t}(t)$ can significantly affect the sample size $n S$ required for $\hat{E}_{E}\left\{E_{A}\left[D_{D}\left(\tau \mid \mathbf{a}, \mathbf{e}_{D}\right) \mid \mathbf{e}_{A}\right]\right\}$ to be close to $E_{E}\left\{E_{A}\left[D_{D}\left(\tau \mid \mathbf{a}, \mathbf{e}_{D}\right) \mid \mathbf{e}_{A}\right]\right\}$. However, any choice for $d_{t}(t)$ will result in approximations that approach $E_{E}\left\{E_{A}\left[D_{D}(\tau \mid \mathbf{a}\right.\right.$, $\left.\left.\left.\mathbf{e}_{D}\right) \mid \mathbf{e}_{A}\right]\right\}$ if sufficiently large samples are possible.

The implementation of Strategy 3 is now illustrated with the function $D_{D}\left(\tau \mid t, \mathbf{p}, \mathbf{e}_{D}\right)$ and associated definitions for $\mathbf{p}, \mathbf{e}_{A}$ and $\mathbf{e}_{D}$ introduced in conjunction with Eq. (8.3). This illustration uses samples of several different sizes generated from $\mathcal{E} \times \mathscr{P} \times[a, b]$, with the sampling of $\mathbf{p}=\left[a_{1}, a_{2}\right]$ from $\mathscr{P}$ performed in the same manner as described in conjunction with Eqs. (8.25) and (8.26). Further, the time interval $[a, b]=[10,20,000 \mathrm{yr}]$ is used and both uni- 
form and loguniform importance sampling on [10, 20,000 yr] are considered. The outcomes of the sampling process are samples of the form

$$
\begin{aligned}
\mathbf{s}_{i} & =\left[\mathbf{e}_{i}, \mathbf{p}_{i}, t_{i}\right] \\
& =\left[\mathbf{e}_{A i}, \mathbf{e}_{D i}, \mathbf{p}_{i}, t_{i}\right] \\
& =\left[e_{A 1 i}, e_{A 2 i}, e_{A 3 i}, e_{D 1 i}, e_{D 2 i}, \ldots, e_{D 9 i}, a_{1 i}, a_{2 i}, t_{i}\right]
\end{aligned}
$$

for $i=1,2, \ldots, n S$, with the values for $t_{i}$ obtained with either uniform or loguniform sampling depending on the case under consideration.

The function $D_{D}\left(\tau \mid t_{i}, \mathbf{p}_{i}, \mathbf{e}_{D i}\right)$ is evaluated for each element $\mathbf{s}_{i}$ of the sample indicated in Eq. (8.35). For illustration, the results of this evaluation are shown in Figs. 11a and 11c with a linear scale for uniform (Fig. 11a) and loguniform (Fig. 11c) sampling on time, respectively, and in Figs. 12a and 12c with a log scale for uniform (Fig. 12a) and loguniform (Fig. 12c) sampling on time $t$, respectively. Both linear and log scales are used because a linear scale provides a better visual impression of the presence of large values and a log scale permits small values to be accurately observed. The effects of uniform and loguniform sampling on time are clearly visible when the curves in Figs. 11a and 12a, which are obtained with uniform sampling on time, are compared with the curves in Figs. 11c and 12c, which are obtained with loguniform sampling on time. In particular, uniform sampling on time results in a fairly even distribution of start times for nonzero doses; in contrast, loguniform sampling on time results in an obvious skewing of start times for nonzero doses towards early times. However, despite the very different appearances of the dose curves obtained with uniform and loguniform sampling on time, both sets of curves are valid intermediate results in a process leading to an approximation to $E_{E}\left\{E_{A}\left[D_{D}\left(\tau \mid \mathbf{a}, \mathbf{e}_{D}\right) \mid \mathbf{e}_{A}\right]\right\}$ with the relationship in Eq. (8.29).

The approximation of $E_{E}\left\{E_{A}\left[D_{D}\left(\tau \mid \mathbf{a}, \mathbf{e}_{D}\right) \mid \mathbf{e}_{A}\right]\right\}$ in Eq. (8.29) can be viewed as an average of $n S$ weighted doses. In general, the weight $w_{i}$ is given by

$$
w_{i}=\lambda_{D i} / d_{t}\left(t_{i}\right)
$$

for $i=1,2, \ldots, n S$; more specifically, the weight is given by

$$
w_{i}=\lambda_{D i}(b-a)
$$

for uniform sampling on time and by

$$
w_{i}=\lambda_{D i} t_{i} \ln (b / a)
$$

for loguniform sampling on time. In turn, the weighted doses $w D_{i}$ are given by

$$
\begin{aligned}
w D_{i} & =w_{i} D_{D}\left(\tau \mid t_{i}, \mathbf{p}_{i}, \mathbf{e}_{D i}\right) \\
& =\left[\lambda_{D i} / d_{t}\left(t_{i}\right)\right] D_{D}\left(\tau \mid t_{i}, \mathbf{p}_{i}, \mathbf{e}_{D i}\right) \\
& = \begin{cases}\lambda_{D i}(b-a) D_{D}\left(\tau \mid t_{i}, \mathbf{p}_{i}, \mathbf{e}_{D i}\right) & \text { for uniform sampling on time } \\
\lambda_{D i} t_{i} \ln (b / a) D_{D}\left(\tau \mid t_{i}, \mathbf{p}_{i}, \mathbf{e}_{D i}\right) & \text { for loguniform sampling on time }\end{cases}
\end{aligned}
$$

for $i=1,2, \ldots, n S$.

Weighted results for the example under consideration are shown in Figs. 11b and 11d with a linear scale and uniform (Fig. 11b) and loguniform (Fig. 11d) sampling on time and in Figs. 12b and 12d for the same results but with a log scale. The inclusion of the weight $w_{i}$ distorts the actual dose. In particular, the weight incorporates the 


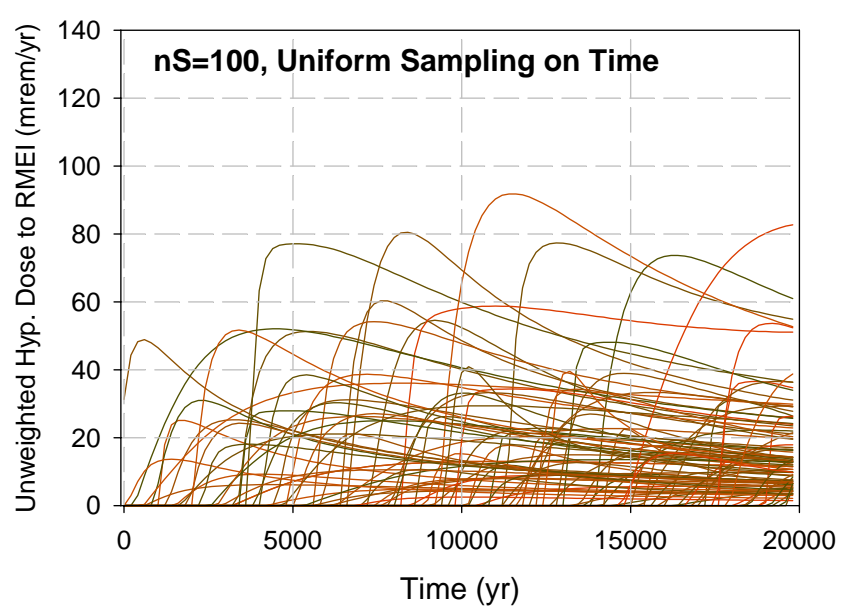

(a)

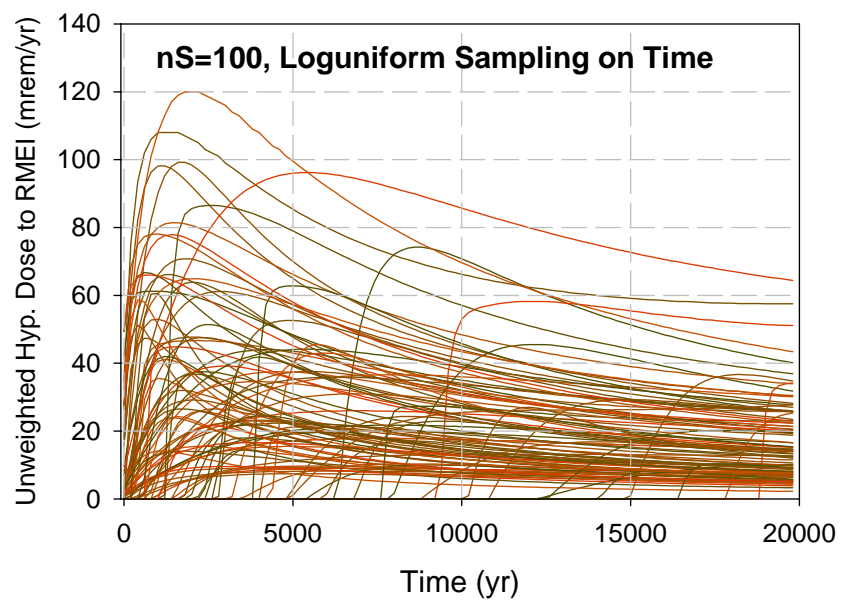

(c)

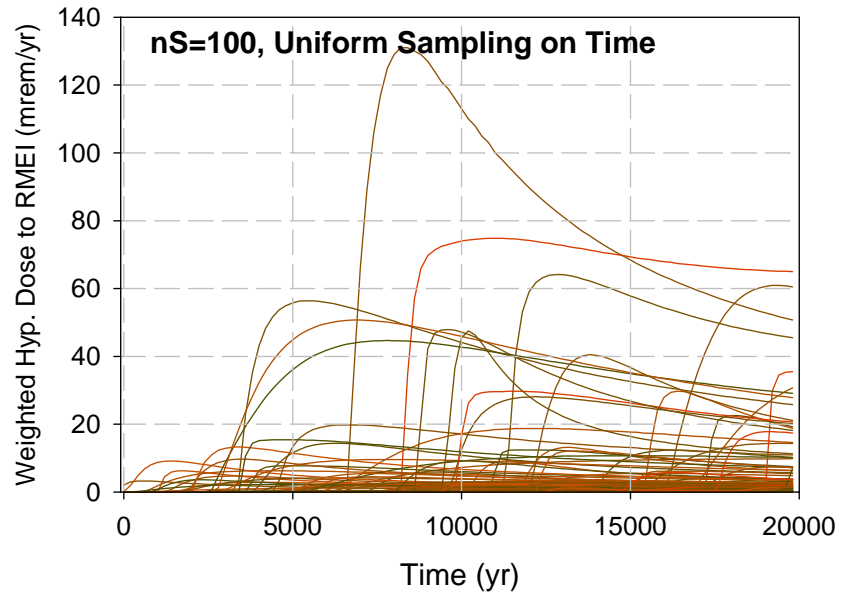

(b)

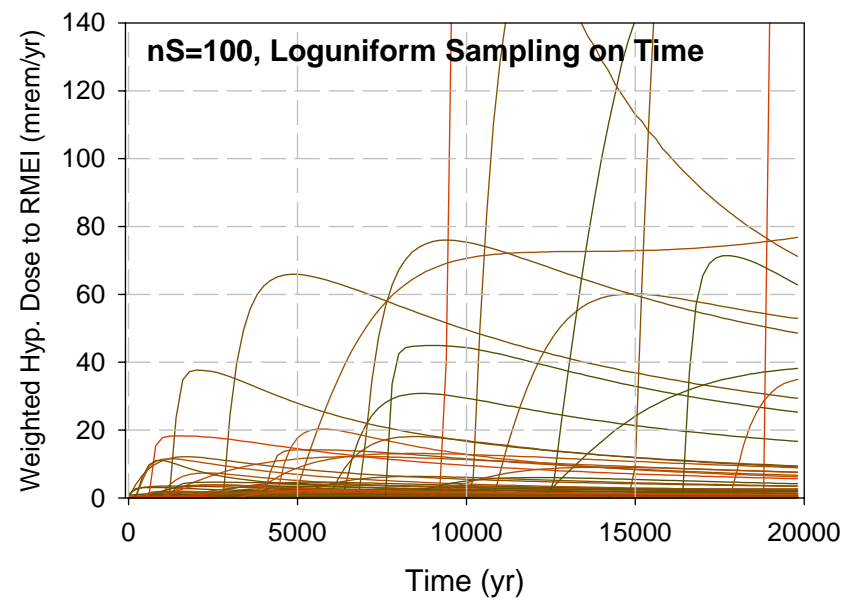

(d)

Fig. 11. Linear scale plots for unweighted and weighted hypothetical doses obtained with samples of size $n S=100$ of the form indicated in Eq. (8.35) and used in the approximation of expected (combined mean) dose $E_{E}\left\{E_{A}\left[D_{D}\left(\tau \mid \mathbf{a}, \mathbf{e}_{D}\right) \mid \mathbf{e}_{A}\right]\right\}$ defined in Eq. (8.29) with Strategy 3: (a) $D_{D}\left(\tau \mid t_{i}, \mathbf{p}_{i}, \mathbf{e}_{D i}\right), i=1,2, \ldots, 100$, obtained with uniform sampling on time, (b) $\left[(10,000-10) \lambda_{D i}\right] D_{D}\left(\tau \mid t_{i}, \mathbf{p}_{i}, \mathbf{e}_{D i}\right), i=1,2, \ldots, 100$, obtained with uniform sampling on time, (c) $D_{D}\left(\tau \mid t_{i}, \mathbf{p}_{i}, \mathbf{e}_{D i}\right), i=1,2, \ldots, 100$, obtained with loguniform sampling on time, and (d) $\left[\lambda_{D i} t_{i} \ln (10,000 / 10)\right] D_{D}\left(\tau \mid t_{i}, \mathbf{p}_{i}, \mathbf{e}_{D i}\right), i=1,2, \ldots, 100$, obtained with loguniform sampling on time.

uncertain rate $\lambda_{D}$ and also the effects of the particular importance sampling distribution selected for use. For example, loguniform sampling results in many small values for time and few large values for time (e.g., see Fig. 12c). To correct for this, the factor $t_{i} \ln (b / a)$ in the weight for loguniform sampling is small for small values of $t$ and large for large values of $t$. The effect of this can be seen in the upward scaling of doses in Figs. 11d and 12d for events that initiate late in time. As a result, although weighted doses are valid intermediate results in the approximation of $E_{E}\left\{E_{A}\left[D_{D}\left(\tau \mid \mathbf{a}, \mathbf{e}_{D}\right) \mid \mathbf{e}_{A}\right]\right\}$ in Eq. (8.29), it is difficult to give a useful uncertainty interpretation to distributions of weighted doses of the form shown in Figs. 11b, 11d, 12b and 12d.

As for Strategies 1 and 2, replicated sampling can be used to assess the stability of estimates for $E_{E}\left\{E_{A}\left[D_{D}(\tau \mid \mathbf{a}\right.\right.$, $\left.\left.\left.\mathbf{e}_{D}\right) \mid \mathbf{e}_{A}\right]\right\}$ obtained with Strategy 3 (Fig. 13). Considerable variability is present in the estimates of $E_{E}\left\{E_{A}\left[D_{D}(\tau \mid \mathbf{a}\right.\right.$, $\left.\left.\left.\mathbf{e}_{D}\right) \mid \mathbf{e}_{A}\right]\right\}$ for $n S=100$, with this variability steadily decreasing as the sample size increases. The effects of uniform and loguniform importance sampling on time can be seen in the variability associated with the estimates for $E_{E}\left\{E_{A}\left[D_{D}\left(\tau \mid \mathbf{a}, \mathbf{e}_{D}\right) \mid \mathbf{e}_{A}\right]\right\}$. Specifically, estimates obtained with uniform sampling are more variable at early times 


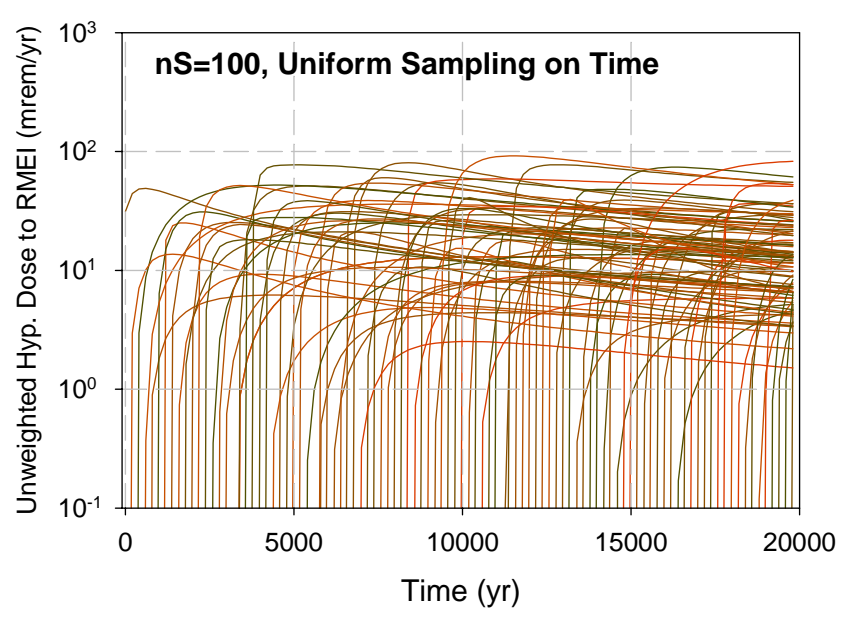

(a)

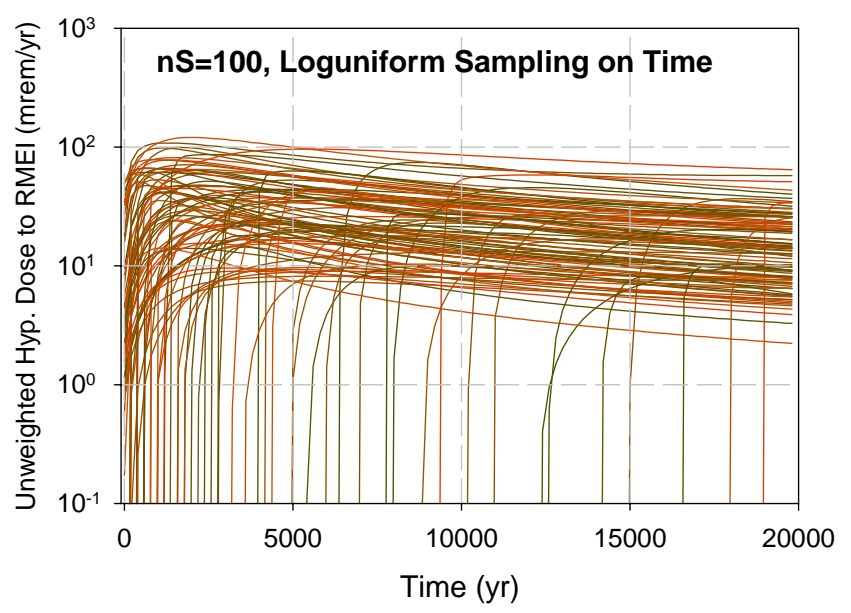

(c)

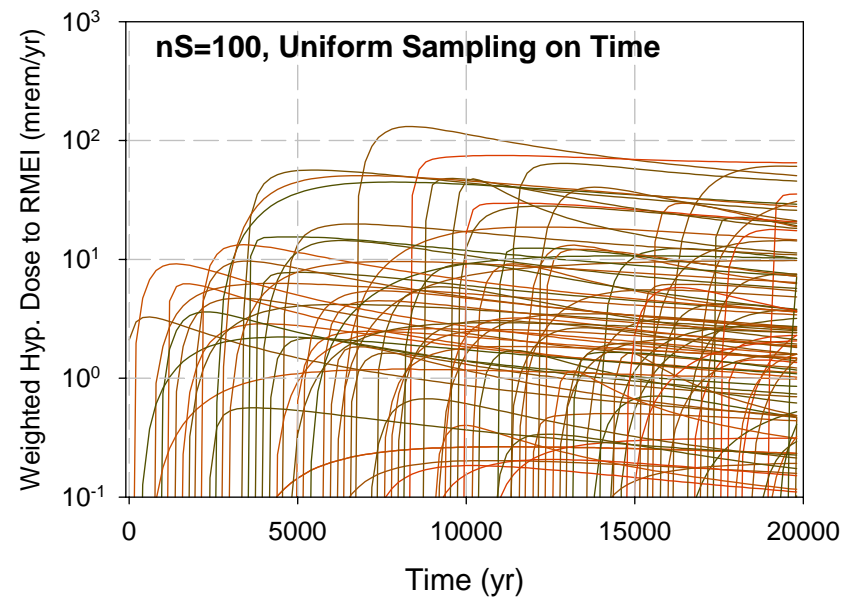

(b)

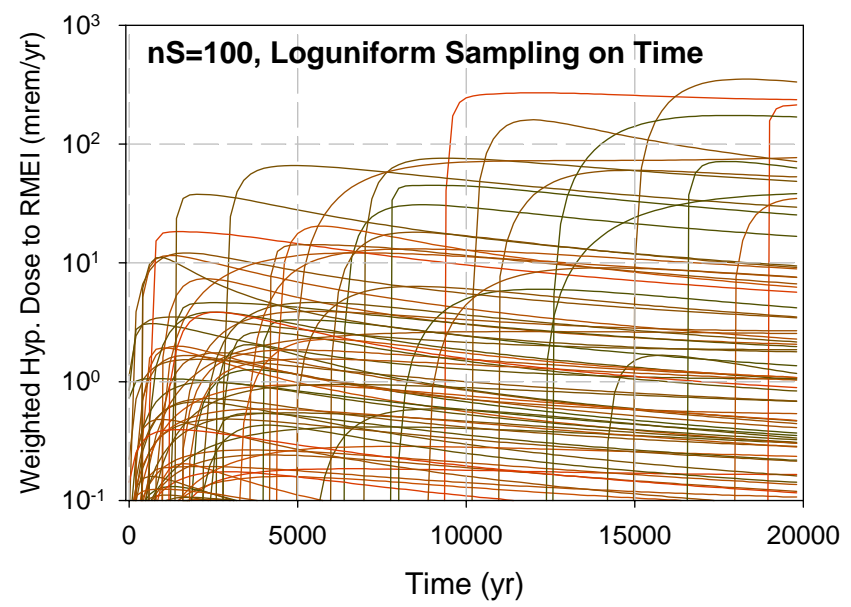

(d)

Fig. 12. Log scale plots for unweighted and weighted hypothetical doses obtained with same samples of size $n S=$ 100 of the form indicated in Eq. (8.35) and used in the approximation of expected (combined mean) dose $E_{E}\left\{E_{A}\left[D_{D}\left(\tau \mid \mathbf{a}, \mathbf{e}_{D}\right) \mid \mathbf{e}_{A}\right]\right\}$ defined in Eq. (8.29) with Strategy 3: (a) $D_{D}\left(\tau \mid t_{i}, \mathbf{p}_{i}, \mathbf{e}_{D i}\right), i=1,2, \ldots, 100$, obtained with uniform sampling on time, (b) $\left[(10,000-10) \lambda_{D i}\right] D_{D}\left(\tau \mid t_{i}, \mathbf{p}_{i}, \mathbf{e}_{D i}\right), i=1,2, \ldots, 100$, obtained with uniform sampling on time, (c) $D_{D}\left(\tau \mid t_{i}, \mathbf{p}_{i}, \mathbf{e}_{D i}\right), i=1,2, \ldots, 100$, obtained with loguniform sampling on time, and (d) $\left[\lambda_{D i} t_{i} \ln (10,000 / 10)\right] D_{D}\left(\tau \mid t_{i}, \mathbf{p}_{i}, \mathbf{e}_{D i}\right), i=1,2, \ldots, 100$, obtained with loguniform sampling on time.

than results obtained with loguniform sampling and less variable at later times than results obtained with loguniform sampling. This pattern of relative variability results because loguniform sampling results in more small values for $t_{i}$ than uniform sampling and, in contrast, uniform sampling results in more large values for $t_{i}$ than loguniform sampling. For $n S=5000$, the results with uniform and loguniform sampling on time are very similar. Thus, even though the distributions of individual dose curves look very different for uniform and loguniform sampling on time, these two sampling procedures are leading to similar estimates for $E_{E}\left\{E_{A}\left[D_{D}\left(\tau \mid \mathbf{a}, \mathbf{e}_{D}\right) \mid \mathbf{e}_{A}\right]\right\}$.

The replicated results in Fig. 13 can also be used to determine confidence intervals associated with estimates for $E_{E}\left\{E_{A}\left[D_{D}\left(\tau \mid \mathbf{a}, \mathbf{e}_{D}\right) \mid \mathbf{e}_{A}\right]\right\}$ as indicated in conjunction with Eqs. (7.16) - (7.19) (Fig. 14). These confidence intervals decrease in width as the sample size $n S$ increases and are almost vanishingly small for $n S=5000$. The previously observed property of loguniform sampling to give more stable results at early times and uniform sampling to give more stable results at later times can be seen in the smaller confidence intervals at early times for loguniform 
(a)

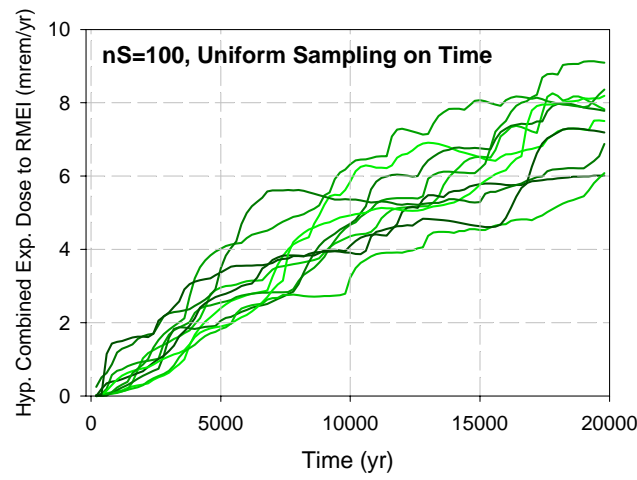

(c)

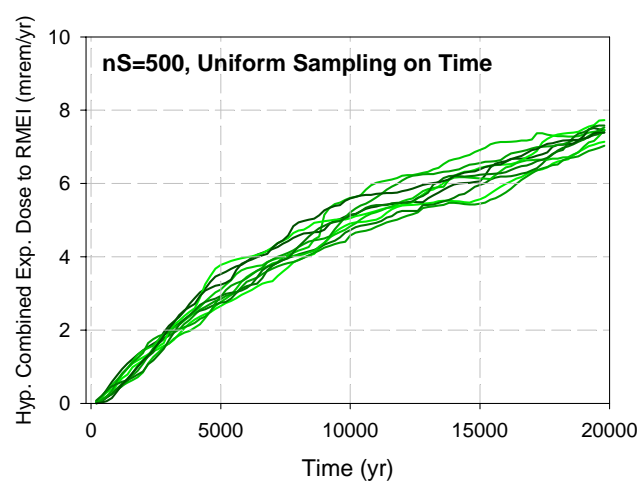

(e)

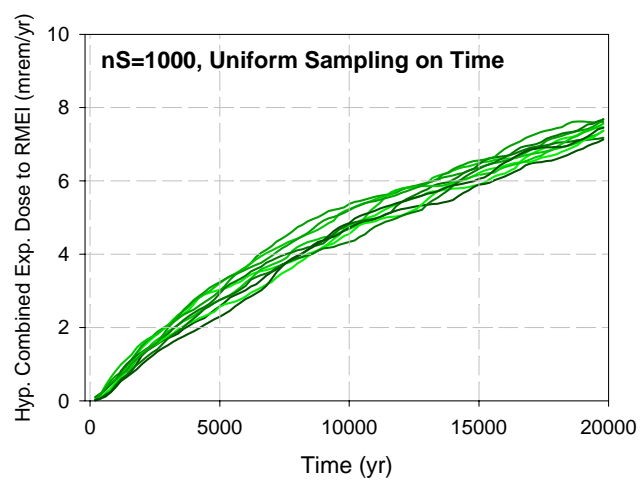

(g)

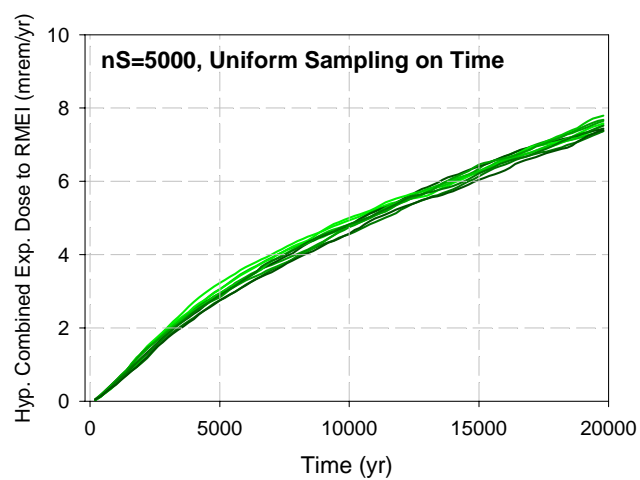

(b)

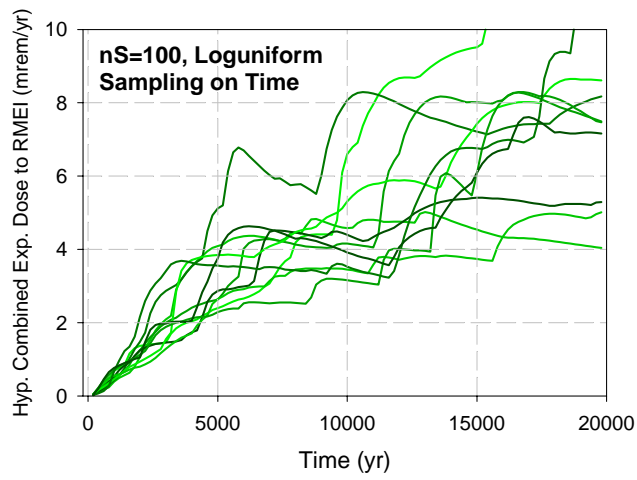

(d)

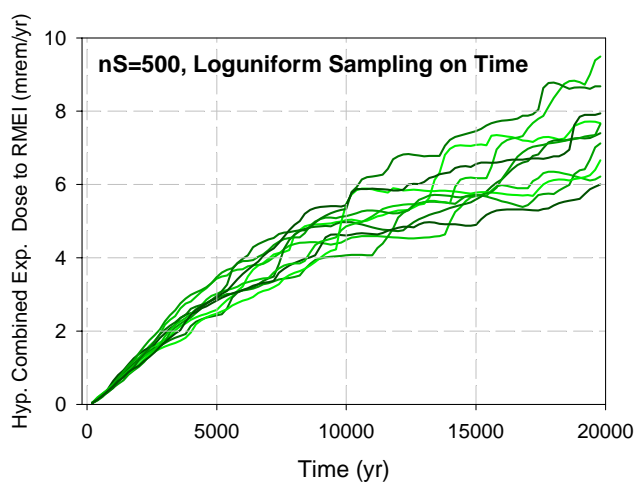

(f)

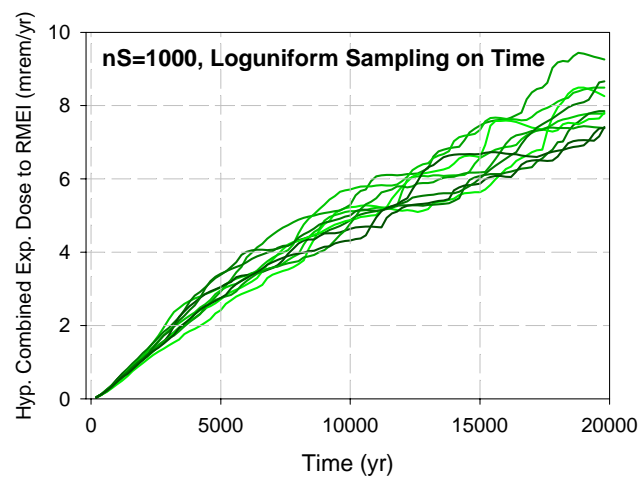

(h)

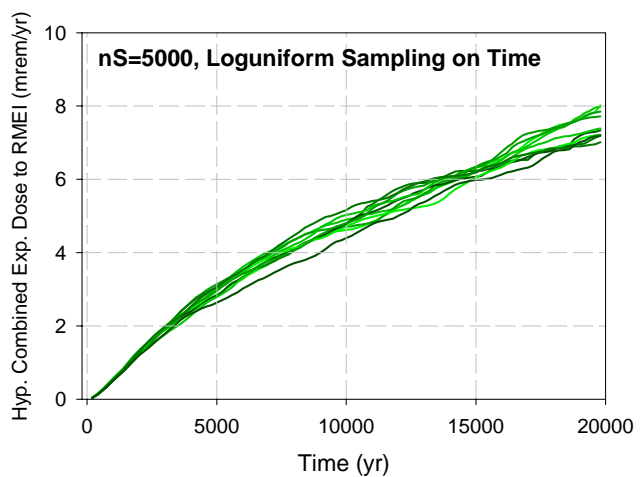

Fig. 13. Representation of sampling variability that results from using $n R=10$ replicated LHSs of size $n S=100$, 500, 1000 and 5000 in the hypothetical estimation of expected (combined mean) dose $E_{E}\left\{E_{A}\left[D_{D}(\tau \mid \mathbf{a}\right.\right.$, $\left.\left.\left.\mathbf{e}_{D}\right) \mid \mathbf{e}_{A}\right]\right\}$ with Strategy 3 and either uniform (a,c,e,g) or loguniform (b,d,f,h) sampling on time. 
(a)

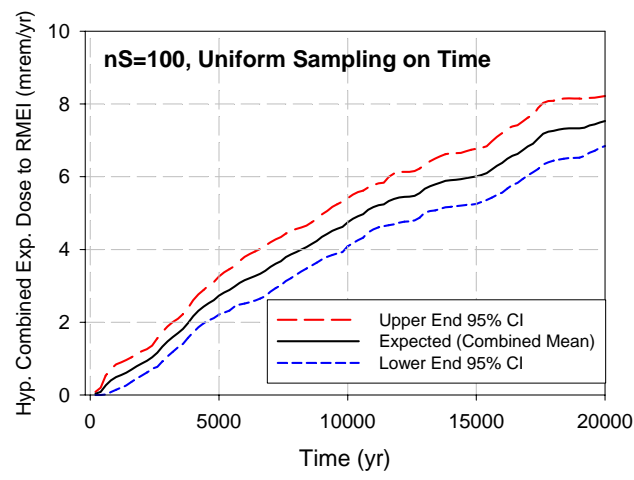

(c)

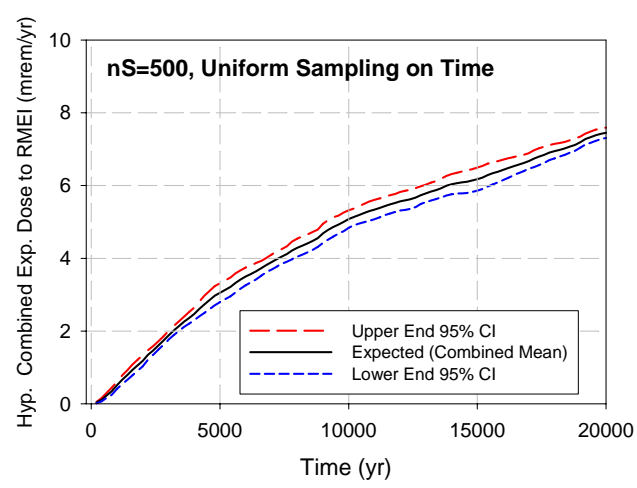

(e)

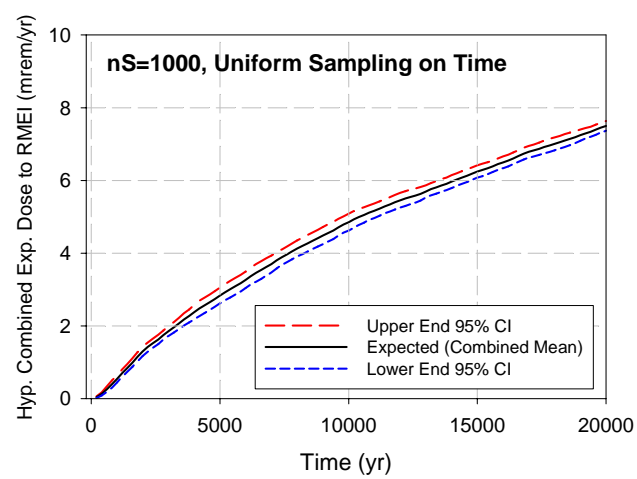

(g)

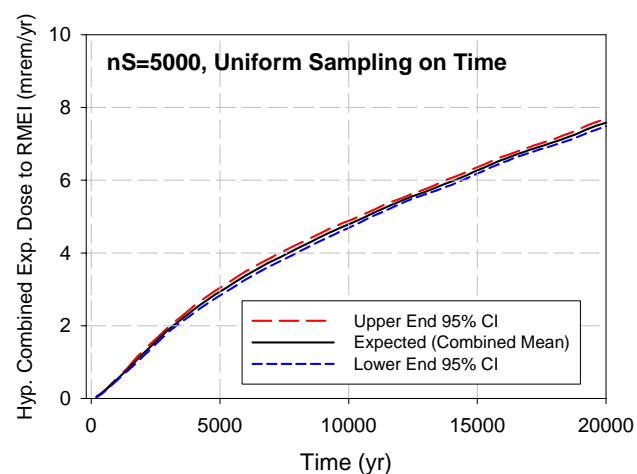

(b)

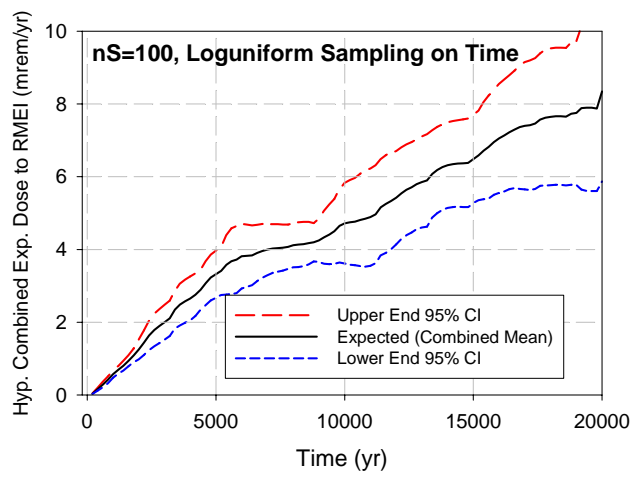

(d)

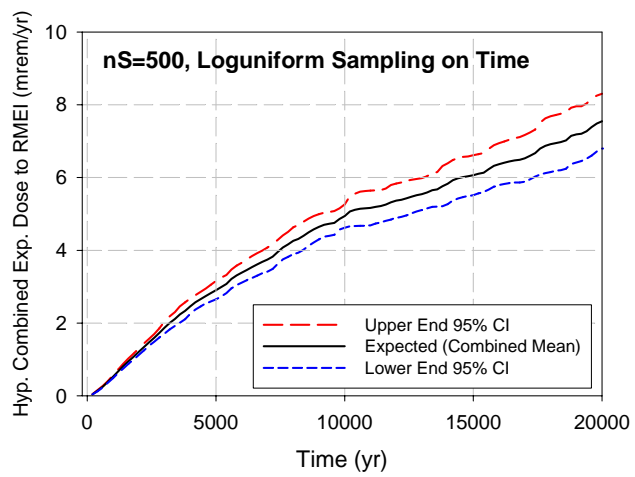

(f)

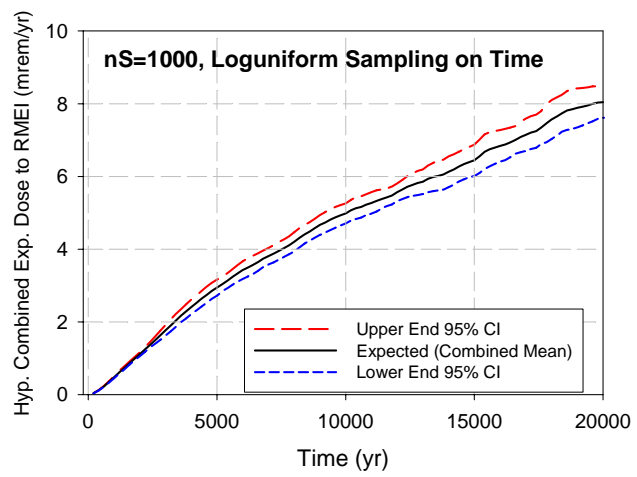

(h)

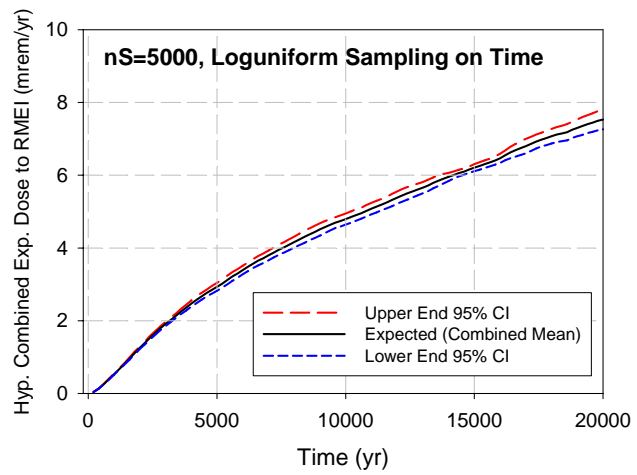

Fig. 14. Ninety-five percent (i.e., 95\%) confidence intervals (CIs) for hypothetical estimates of expected (combined mean) dose $E_{E}\left\{E_{A}\left[D_{D}\left(\tau \mid \mathbf{a}, \mathbf{e}_{D}\right) \mid \mathbf{e}_{A}\right]\right\}$ with Strategy 3 that result from using $n R=10$ replicated LHSs of sizes $n S=100,500,1000$ and 5000 and either uniform (a,c,e,g) or loguniform (b,d,f,h) sampling on time. 
sampling. However, this is a property of the example analysis under consideration; an analysis with different properties could show different patterns of behavior. In general, the selection of an effective importance sampling procedure must be based on properties of the particular analysis under consideration.

As for Strategies 1 and 2, the confidence intervals in Fig. 14 for estimates of $E_{E}\left\{E_{A}\left[D_{D}\left(\tau \mid \mathbf{a}, \mathbf{e}_{D}\right) \mid \mathbf{e}_{A}\right]\right\}$ obtained with Strategy 3 can be used to assess compliance with the acceptance criterion in Quote (YMRP4). However, as is also the case for Strategy 2, estimates of the epistemic uncertainty associated with expected dose over aleatory uncertainty (i.e., $\left.E_{A}\left[D_{D}\left(\tau \mid \mathbf{a}, \mathbf{e}_{D}\right) \mid \mathbf{e}_{A}\right]\right)$ are not possible. Thus, with Strategy 3, results associated with only one of the two possible interpretations of the acceptance criterion in Quote (YMRP5) discussed at the end of Sect. 8.1 are obtainable.

Although the intermediate results can look very different, Strategies 1, 2 and 3 are all providing estimates for $E_{E}\left\{E_{A}\left[D_{D}\left(\tau \mid \mathbf{a}, \mathbf{e}_{D}\right) \mid \mathbf{e}_{A}\right]\right\}$. The similarity of these estimates can be seen by comparing the estimates for $E_{E}\left\{E_{A}\left[D_{D}\left(\tau \mid \mathbf{a}, \mathbf{e}_{D}\right) \mid \mathbf{e}_{A}\right]\right\}$ in Figs. 7, 10 and 14. 


\section{Expected Dose Conditional on Single Disruption}

It is natural to ask what uncertainty interpretation, if any, can be given to the distributions of dose curves in Figs. 11a, 11c, 12a and 12c. As it turns out, there is a natural interpretation of the dose curves in Figs. 11a and 12a, which are obtained with uniform sampling on time, that derives from the consideration of a Poisson process with a constant occurrence rate $\lambda_{D}$. Such a process is equally likely to occur at any given time. As a result, if the assumption is made that the process has occurred exactly once in a time interval $[a, b]$, then the possible times at which this process has occurred has a uniform distribution on $[a, b]$ as indicated in conjunction with Eq. (4.12). This is the distribution defined in Eq. (8.31) and used in the generation of the dose curves in Figs. 11a and 12a. As a result, the distribution of dose curves in Figs. 11a and 12a is displaying the uncertainty in dose conditional on the assumption that exactly one disruption has occurred in the time interval $[a, b]=[10,20,000 \mathrm{yr}]$, with the displayed uncertainty deriving from both epistemic uncertainty in $\mathbf{e}=\left[\mathbf{e}_{A}, \mathbf{e}_{D}\right]$ and aleatory uncertainty in $t$ and $\mathbf{p}=\left[a_{1}, a_{2}\right]$.

Results conditional on the occurrence of exactly one disruption in the time interval $[a, b]$ are now considered in more detail. In particular, doses of the form $D_{D}\left(\tau \mid \mathbf{a}, \mathbf{e}_{D}\right)$ for $\mathbf{a}$ belonging to the set $\mathcal{A}_{1}(a, b)$ defined in Eq. (5.6) are under consideration. The expected value for $D_{D}\left(\tau \mid \mathbf{a}, \mathbf{e}_{D}\right)$ over aleatory uncertainty conditional on a fixed element $\mathbf{e}$ $=\left[\mathbf{e}_{A}, \mathbf{e}_{D}\right]$ of $\mathcal{E}$ and $\mathbf{a} \in \mathcal{A}_{1}(a, b)$ is given by

$$
\begin{aligned}
E_{A}\left[D_{D}\left(\tau \mid \mathbf{a}, \mathbf{e}_{D}\right) \mid \mathbf{e}_{A}, \mathbf{a} \in \mathcal{A}_{1}(a, b)\right] & =\int_{a}^{\tau} \int_{\mathcal{P}} D_{D}\left(\tau \mid t, \mathbf{p}, \mathbf{e}_{D}\right) d_{P}\left(\mathbf{p} \mid \mathbf{e}_{A}\right) d_{t, u}(t) \mathrm{d} P \mathrm{~d} t \\
& =\int_{a}^{b} \int_{\mathcal{P}} D_{D}\left(\tau \mid t, \mathbf{p}, \mathbf{e}_{D}\right) d_{P}\left(\mathbf{p} \mid \mathbf{e}_{A}\right) d_{t, u}(t) \mathrm{d} P \mathrm{~d} t,
\end{aligned}
$$

with the uniform density $d_{t, u}(t)$ used because the expectation is conditional on exactly one occurrence in the time interval $[a, b]$ (i.e., on $\mathbf{a} \in \mathcal{A}_{1}(a, b)$ ) and the integral over time rewritten from $a$ to $b$ rather than from $a$ to $\tau$ because $D_{D}\left(\tau \mid t, \mathbf{p}, \mathbf{e}_{D}\right)=0$ for $\tau<t$.

In turn, the expectation for $E_{A}\left[D_{D}\left(\tau \mid \mathbf{a}, \mathbf{e}_{D}\right) \mid \mathbf{e}_{A}, \mathbf{a} \in \mathcal{A}_{1}(a, b)\right]$ over epistemic uncertainty is given by

$$
\begin{aligned}
E_{E}\left\{E_{A}[\right. & \left.\left.D_{D}\left(\tau \mid \mathbf{a}, \mathbf{e}_{D}\right) \mid \mathbf{e}_{A}, \mathbf{a} \in \mathcal{A}_{1}(a, b)\right]\right\}=\int_{\mathcal{E}} E_{A}\left[D_{D}\left(\tau \mid \mathbf{a}, \mathbf{e}_{D}\right) \mid \mathbf{e}_{A}, \mathbf{a} \in \mathcal{A}_{1}(a, b)\right] d_{E}(\mathbf{e}) \mathrm{d} E \\
= & \int_{\mathcal{E}}\left\{\int_{a}^{\tau} \int_{\mathcal{R}} D_{D}\left(\tau \mid t, p, \mathbf{e}_{D}\right) d_{P}\left(\mathbf{p} \mid \mathbf{e}_{A}\right) d_{t, u}(t) \mathrm{d} P \mathrm{~d} t\right\} d_{E}(\mathbf{e}) \mathrm{d} E \\
& =\int_{\mathcal{E}} \int_{a}^{b} \int_{\mathcal{P}} D_{D}\left(\tau \mid t, \mathbf{p}, \mathbf{e}_{D}\right) d_{P}\left(\mathbf{p} \mid \mathbf{e}_{A}\right) d_{t, u}(t) d_{E}(\mathbf{e}) \mathrm{d} P \mathrm{~d} t \mathrm{~d} E
\end{aligned}
$$

A better notation for the preceding result is perhaps

$$
E_{E \cup A}\left[D_{D}(\tau) \mid \mathbf{a} \in \mathcal{A}_{1}(a, b)\right]=\int_{\mathcal{E}} \int_{a}^{b} \int_{\mathcal{P}} D_{D}\left(\tau \mid t, \mathbf{p}, \mathbf{e}_{D}\right) d_{P}\left(\mathbf{p} \mid \mathbf{e}_{A}\right) d_{t, u}(t) d_{E}(\mathbf{e}) \mathrm{d} P \mathrm{~d} t \mathrm{~d} E,
$$

which indicates that the expectation is over both epistemic and aleatory uncertainty and conditional on $\mathbf{a} \in \mathcal{A}_{1}(a, b)$. Similarly,

$$
p_{E \cup A}\left[D_{D}(\tau) \leq D \mid \mathbf{a} \in \mathcal{A}_{1}(a, b)\right]=\int_{\mathcal{E}} \int_{a}^{b} \int_{\mathcal{P}} \underline{\delta}_{D}\left[D_{D}\left(\tau \mid t, \mathbf{p}, \mathbf{e}_{D}\right)\right] d_{P}\left(\mathbf{p} \mid \mathbf{e}_{A}\right) d_{t, u}(t) d_{E}(\mathbf{e}) \mathrm{d} P \mathrm{~d} t \mathrm{~d} E
$$

is the probability deriving from both epistemic and aleatory uncertainty and conditional on $\mathbf{a} \in \mathcal{A}_{1}(a, b)$ that a dose less than $D$ will occur.

Because of the presence of the density functions $d_{P}\left(\mathbf{p} \mid \mathbf{e}_{A}\right), d_{t, u}(t)$ and $d_{E}(\mathbf{e})$, the integrals in Eqs. (9.3) and (9.4) are defining expected values for $D_{D}\left(\tau \mid t, \mathbf{p}, \mathbf{e}_{D}\right)$ and $\underline{\delta}_{D}\left[D_{D}\left(\tau \mid t, \mathbf{p}, \mathbf{e}_{D}\right)\right]$. As a result, samples generated in consis- 
tency with these density functions can be used to produce approximations to $E_{E \cup A}\left[D_{D}(\tau) \mid \mathbf{a} \in \mathcal{A}_{1}(a, b)\right]$ and $p_{E \cup A}\left[D_{D}(\tau) \leq D \mid \mathbf{a} \in \mathcal{A}_{1}(a, b)\right]$ of the form

$$
\hat{E}_{E \cup A}\left[D_{D}(\tau) \mid \mathbf{a} \in \mathcal{A}_{1}(a, b)\right]=\sum_{i=1}^{n S} D_{D}\left(\tau \mid t_{i}, \mathbf{p}_{i}, \mathbf{e}_{D i}\right) / n S
$$

and

$$
\hat{p}_{E \cup A}\left[D_{D}(\tau) \leq D \mid \mathbf{a} \in \mathcal{A}_{1}(a, b)\right]=\sum_{i=1}^{n S} \underline{\delta}_{D}\left[D_{D}\left(\tau \mid t_{i}, \mathbf{p}_{i}, \mathbf{e}_{D i}\right)\right] / n S .
$$

The samples indicated in Eq. (8.35) generated with uniform sampling on time (i.e., with the density function $d_{t, u}(t)$ ) are of this form and thus can be used in the preceding approximations for $E_{E \cup A}\left[D_{D}(\tau) \mid \mathbf{a} \in \mathcal{A}_{1}(a, b)\right]$ and $p_{E \cup A}\left[D_{D}(\tau) \leq D \mid \mathbf{a} \in \mathcal{A}_{1}(a, b)\right]$. Thus, results of the form shown in Figs. 11a and 12a obtained with uniform sampling on time can be used to estimate $E_{E \cup A}\left[D_{D}(\tau) \mid \mathbf{a} \in \mathcal{A}_{1}(a, b)\right]$ and $p_{E \cup A}\left[D_{D}(\tau) \leq D \mid \mathbf{a} \in \mathcal{A}_{1}(a, b)\right]$. Such approximations for $n S=1000$ and 5000 are shown in Figs. 15a and 15c.

Results obtained when time is not sampled uniformly on $[a, b]$ can also be used to estimate $E_{E \cup A}\left[D_{D}(\tau) \mid \mathbf{a} \in\right.$ $\left.\mathcal{A}_{1}(a, b)\right]$ and $p_{E \cup A}\left[D_{D}(\tau) \leq D \mid \mathbf{a} \in \mathcal{A}_{1}(a, b)\right]$. However, a correction must be introduced to account for the nonuniform sampling on time. For $E_{E \cup A}\left[D_{D}(\tau) \mid \mathbf{a} \in \mathcal{A}_{1}(a, b)\right]$, this correction is obtained from the reformulation of Eq. (9.3) as

$$
\begin{aligned}
E_{E \cup A}\left[D_{D}(\tau) \mid \mathbf{a} \in \mathcal{A}_{1}(a, b)\right] & =\int_{\mathcal{E}} \int_{a}^{b} \int_{\mathcal{P}}\left[\frac{D_{D}\left(\tau \mid t, \mathbf{p}, \mathbf{e}_{D}\right) d_{t, u}(t)}{d_{t}(t)}\right] d_{P}\left(\mathbf{p} \mid \mathbf{e}_{A}\right) d_{t}(t) d_{E}(\mathbf{e}) \mathrm{d} P \mathrm{~d} t \mathrm{~d} E \\
& =\int_{\mathcal{E}} \int_{a}^{b} \int_{\mathcal{P}}\left[\frac{D_{D}\left(\tau \mid t, \mathbf{p}, \mathbf{e}_{D}\right)}{(b-a) d_{t}(t)}\right] d_{P}\left(\mathbf{p} \mid \mathbf{e}_{A}\right) d_{t}(t) d_{E}(\mathbf{e}) \mathrm{d} P \mathrm{~d} t \mathrm{~d} E
\end{aligned}
$$

where $d_{t}(t)$ is an arbitrary density function for $t$ defined on $[a, b]$ (e.g., $d_{t, l n}(t)$ defined in Eq. (8.32)). Similarly, the definition for $p_{E \cup A}\left[D(\tau) \leq D \mid \mathbf{a} \in \mathcal{A}_{1}(a, b)\right]$ in Eq. (9.4) can be reformulated as

$$
\begin{aligned}
p_{E \cup A}\left[D_{D}(\tau) \leq D \mid \mathbf{a} \in \mathcal{A}_{1}(a, b)\right] & =\int_{\mathcal{E}} \int_{a}^{b} \int_{\mathcal{P}}\left[\frac{\delta_{D}\left[D_{D}\left(\tau \mid t, \mathbf{p}, \mathbf{e}_{D}\right)\right] d_{t, u}(t)}{d_{t}(t)}\right] d_{P}\left(\mathbf{p} \mid \mathbf{e}_{A}\right) d_{t}(t) d_{E}(\mathbf{e}) \mathrm{d} P \mathrm{~d} t \mathrm{~d} E \\
& =\int_{\mathcal{E}} \int_{a}^{b} \int_{\mathcal{P}}\left[\frac{\delta_{D}\left[D_{D}\left(\tau \mid t, \mathbf{p}, \mathbf{e}_{D}\right)\right]}{(b-a) d_{t}(t)}\right] d_{P}\left(\mathbf{p} \mid \mathbf{e}_{A}\right) d_{t}(t) d_{E}(\mathbf{e}) \mathrm{d} P \mathrm{~d} t \mathrm{~d} E
\end{aligned}
$$

The preceding reformulations can now be used to estimate $E_{E \cup A}\left[D_{D}(\tau) \mid \mathbf{a} \in \mathcal{A}_{1}(a, b)\right]$ and $p_{E \cup A}\left[D_{D}(\tau) \leq D \mid \mathbf{a} \in\right.$ $\left.\mathcal{A}_{1}(a, b)\right]$.

Specifically, generation of a sample of the form indicated in Eq. (8.35) in consistency with the density functions $d_{P}\left(\mathbf{p} \mid \mathbf{e}_{A}\right), d_{t}(t)$ and $d_{E}(\mathbf{e})$ results in the approximations

$$
\hat{E}_{E \cup A}\left[D_{D}(\tau) \mid \mathbf{a} \in \mathcal{A}_{1}(a, b)\right]=\sum_{i=1}^{n S}\left[\frac{D_{D}\left(\tau \mid t_{i}, \mathbf{p}_{i}, \mathbf{e}_{D i}\right)}{(b-a) d_{t}\left(t_{i}\right)}\right] / n S
$$

and 


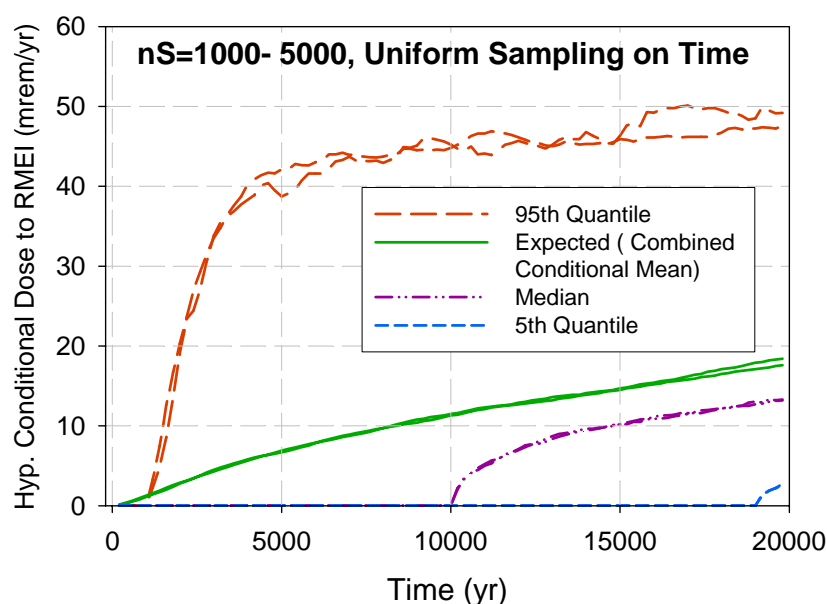

(a)

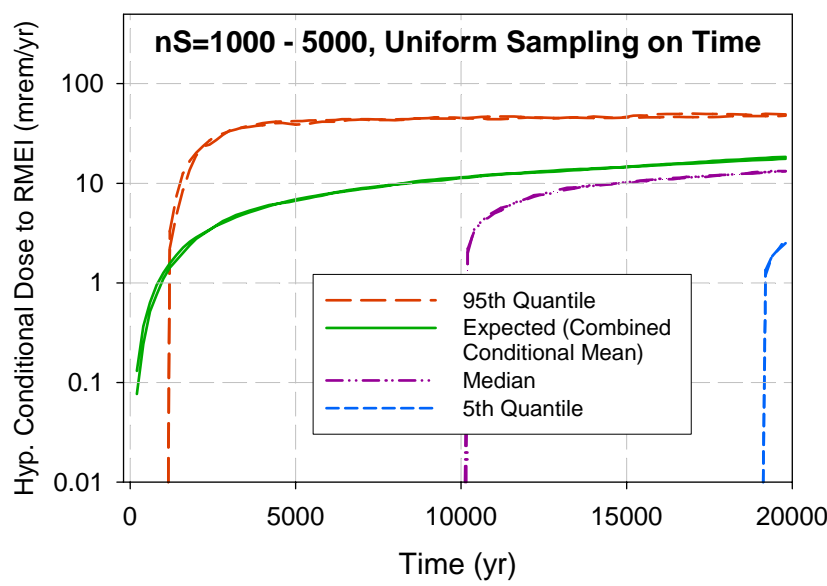

(c)

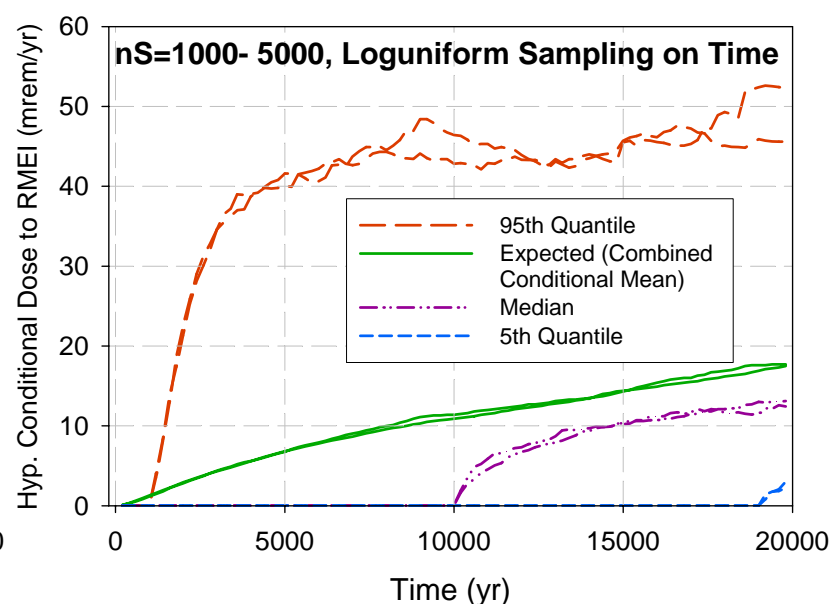

(b)

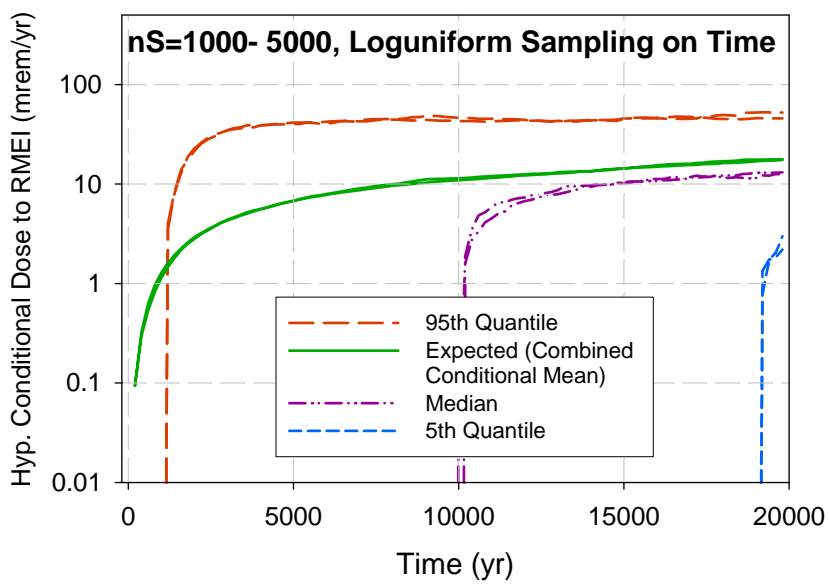

(d)

Fig. 15. Combined epistemic and aleatory uncertainty in hypothetical dose conditional on exactly one disruptive occurrence taking place in the time interval $[a, b]=[10,20,000 \mathrm{yr}]$ summarized as expected (combined conditional mean) dose $\hat{E}_{E \cup A}\left[D_{D}(\tau) \mid \mathbf{a} \in \mathcal{A}_{1}(a, b)\right]$ and associated quantiles (i.e., 0.05, 0.5, 0.95) for $D_{D}\left(\tau \mid t, \mathbf{p}, \mathbf{e}_{D}\right)$ conditional on $\mathbf{a}=[t, \mathbf{p}] \in \mathcal{A}_{1}(a, b)$ defined by $\hat{p}_{E \cup A}\left[D_{D}(\tau)<D \mid \mathbf{a} \in \mathcal{A}_{1}(a, b)\right]$ obtained with Strategy 3 for the evaluation of expected (combined mean) dose $E_{E}\left\{E_{A}\left[D_{D}\left(\tau \mid \mathbf{a}, \mathbf{e}_{D}\right) \mid \mathbf{e}_{A}\right]\right\}$ and LHSs of size $n S=1000$ and 5000: (a,c) Uniform sampling on time and use of approximations in Eqs. (9.5) and (9.6), and (b,d) Loguniform sampling on time and use of approximations in Eqs. (9.9) and (9.10).

$$
\hat{p}_{E \cup A}\left[D_{D}(\tau) \leq D \mid \mathbf{a} \in \mathcal{A}_{1}(a, b)\right]=\sum_{i=1}^{n S}\left[\frac{\delta_{D}\left[D_{D}\left(\tau \mid t_{i}, \mathbf{p}_{i}, \mathbf{e}_{D i}\right)\right]}{(b-a) d_{t}\left(t_{i}\right)}\right] / n S
$$

to $E_{E \cup A}\left[D_{D}(\tau) \mid \mathbf{a} \in \mathcal{A}_{1}(a, b)\right]$ and $p_{E \cup A}\left[D_{D}(\tau) \leq D \mid \mathbf{a} \in \mathcal{A}_{1}(a, b)\right]$. Thus, the necessary correction factor $c_{i}$ to permit estimates for $E_{E \cup A}\left[D_{D}(\tau) \mid \mathbf{a} \in \mathcal{A}_{1}(a, b)\right]$ and $p_{E \cup A}\left[D_{D}(\tau) \leq D \mid \mathbf{a} \in \mathcal{A}_{1}(a, b)\right]$ when time is not uniformly sampled is

$$
c_{i}=\frac{1}{(b-a) d_{t}\left(t_{i}\right)}
$$

where $d_{t}(t)$ is the density function used to sample time. For example, 


$$
c_{i}=\frac{t_{i} \ln (b / a)}{b-a}
$$

when time is sampled in consistency with a loguniform distribution on $[a, b]$ (i.e., in consistency with the density function $d_{t, l n}(t)$ defined in Eq. (8.32)).

Results of the form appearing in Figs. 11c and 12c obtained with nonuniform sampling on time can be used to approximate $E_{E \cup A}\left[D_{D}(\tau) \mid \mathbf{a} \in \mathcal{A}_{1}(a, b)\right]$ and $p_{E \cup A}\left[D_{D}(\tau) \leq D \mid \mathbf{a} \in \mathcal{A}_{1}(a, b)\right]$ by use of the correction factor $c_{i}$ defined in Eqs. (9.11) and (9.12) in conjunction with the calculated doses $D_{D}\left(\tau \mid t_{i}, \mathbf{p}_{i}, \mathbf{e}_{D i}\right)$ for the sample under consideration. This results in the approximations in Eqs. (9.9) and (9.10). The outcome of this procedure for loguniform sampling on time and samples of sizes $n S=1000$ and 5000 is illustrated in Figs. 15b and 15d. As comparison of Figs. 15a and 15c with Figs. 15b and 15d shows, uniform sampling on time and loguniform sampling on time lead to similar estimates for the conditional results $E_{E \cup A}\left[D_{D}(\tau) \mid \mathbf{a} \in \mathcal{A}_{1}(a, b)\right]$ and $p_{E \cup A}\left[D_{D}(\tau) \leq D \mid \mathbf{a} \in \mathcal{A}_{1}(a, b)\right]$. However, as indicated by comparison of the dose curves in Figs. 11a and 12a with the dose curves in Figs. 11c and 12c, the distributions of the sampled dose curves are very different in appearance. Thus, although the two approaches for sampling on time produce very different appearing intermediate results, the final results of interest are the same.

As already indicated, the results in Fig. 15 are conditional on exactly one disruptive occurrence taking place in the time interval $[a, b]=[10,20,000 \mathrm{yr}]$. This conditionality assumption has an important but easily overlooked effect. Specifically, the curves in Fig. 15 are functions of the length of the time interval on which the disruptive occurrence is assumed to take place. Because of the uniform distribution of occurrence time, if a time interval [a, $c$ ] was under consideration with $a<c<b$, then the individual curves in Fig. 15 would shift by a factor related to the ratio $(b-a) /(c-a)$ for $a \leq \tau \leq c$. Specifically,

$$
\begin{aligned}
E_{E \cup A}\left[D_{D}(\tau) \mid \mathbf{a} \in \mathcal{A}_{1}(a, c)\right] & =\int_{\mathcal{E}} \int_{a}^{c} \int_{\mathcal{P}}\left[\frac{D_{D}\left(\tau \mid t, \mathbf{p}, \mathbf{e}_{D}\right)}{c-a}\right] d_{P}\left(\mathbf{p} \mid \mathbf{e}_{A}\right) d_{E}(\mathbf{e}) \mathrm{d} P \mathrm{~d} t \mathrm{~d} E \\
& =\left(\frac{b-a}{c-a}\right) \int_{\mathscr{E}} \int_{a}^{\tau} \int_{\mathscr{P}}\left[\frac{D_{D}\left(\tau \mid t, \mathbf{p}, \mathbf{e}_{D}\right)}{b-a}\right] d_{P}\left(\mathbf{p} \mid \mathbf{e}_{A}\right) d_{E}(\mathbf{e}) \mathrm{d} P \mathrm{~d} t \mathrm{~d} E \\
& =\left(\frac{b-a}{c-a}\right) E_{E \cup A}\left[D_{D}(\tau) \mid \mathbf{a} \in \mathcal{A}_{1}(a, b)\right]
\end{aligned}
$$

and, similarly,

$$
p_{E \cup A}\left[D_{D}(\tau) \leq D \mid \mathbf{a} \in \mathcal{A}_{1}(a, c)\right]=\left(\frac{b-a}{c-a}\right) p_{E \cup A}\left[D_{D}(\tau) \leq D \mid \mathbf{a} \in \mathcal{A}_{1}(a, b)\right]
$$

for $a \leq \tau \leq c \leq b$.

The conditional results $E_{E \cup A}\left[D_{D}(\tau) \mid \mathbf{a} \in \mathcal{A}_{1}(a, b)\right]$ and $p_{E \cup A}\left[D_{D}(\tau) \leq D \mid \mathbf{a} \in \mathcal{A}_{1}(a, b)\right]$ can also be estimated with the evaluations of $D_{D}\left(\tau \mid t_{i}, \mathbf{p}_{i}, \mathbf{e}_{D i}\right)$ obtained in the implementation of Strategies 1 and 2 for the estimation of $E_{E}\left\{E_{A}\left[D\left(\tau \mid \mathbf{a}, \mathbf{e}_{D}\right) \mid \mathbf{e}_{A}\right]\right\}$.

As an example for Strategy 1, the results associated with Eq. (8.12) can be used to obtain the approximations

$$
\hat{E}_{E \cup A}\left[D_{D}(\tau) \mid \mathbf{a} \in \mathcal{A}_{1}(a, c)\right]=\sum_{i=1}^{n S} \sum_{j=1}^{n T} \sum_{k=1}^{n A 1} \sum_{l=1}^{n A 2}\left(\frac{m_{i j k l}}{b-a}\right) \Delta a_{2 l} \Delta a_{1 k} \Delta t_{j} / n S
$$

and 


$$
\hat{p}_{E \cup A}\left[D_{D}(\tau) \leq D \mid \mathbf{a} \in \mathcal{A}_{1}(a, c)\right]=\sum_{i=1}^{n S} \sum_{j=1}^{n T} \sum_{k=1}^{n A 1} \sum_{l=1}^{n A 2}\left(\frac{\tilde{m}_{i j k l}}{b-a}\right) \Delta a_{2 l} \Delta a_{1 k} \Delta t_{j} / n S,
$$

where $m_{i j k l}$ is defined in Eq. (8.16) and

$$
\tilde{m}_{i j k l}=\sum_{r=j-1}^{j} \sum_{s=k-1}^{k} \sum_{t=l-1}^{l} \underline{\delta}_{D}\left[D_{D}\left(\tau \mid t_{r}, a_{1 s}, a_{2 t}, \mathbf{e}_{D i}\right)\right] d_{A 1}\left(a_{1 s} \mid e_{A 1 i}\right) d_{A 2}\left(a_{2 t} \mid e_{A 2 i}\right) .
$$

In general, the form of the approximations to $E_{E \cup A}\left[D_{D}(\tau) \mid \mathbf{a} \in \mathcal{A}_{1}(a, b)\right]$ and $p_{E \cup A}\left[D_{D}(\tau) \leq D \mid \mathbf{a} \in \mathcal{A}_{1}(a, b)\right]$ obtained in conjunction with Strategy 1 will depend on the properties of the procedure used to approximate the integral in Eq. (6.6) that defined $E_{A}\left[D\left(\tau \mid \mathbf{a}, \mathbf{e}_{D}\right) \mid \mathbf{e}_{A}\right]$.

As an example for Strategy 2, the results associated with Eq. (8.24) can be used to obtain the approximations

$$
\hat{E}_{E \cup A}\left[D_{D}(\tau) \mid \mathbf{a} \in \mathcal{A}_{1}(a, c)\right]=\sum_{i=1}^{n S} \sum_{j=1}^{n T}\left(\frac{D_{D}\left(\tau \mid t_{j-1}, \mathbf{p}_{i}, \mathbf{e}_{D i}\right)+D_{D}\left(\tau \mid t_{j}, \mathbf{p}_{i}, \mathbf{e}_{D i}\right)}{2(b-a)}\right) / n S
$$

and

$$
\hat{p}_{E \cup A}\left[D_{D}(\tau) \leq D \mid \mathbf{a} \in \mathcal{A}_{1}(a, c)\right]=\sum_{i=1}^{n S} \sum_{j=1}^{n T}\left(\frac{\delta_{D}\left[D_{D}\left(\tau \mid t_{j-1}, \mathbf{p}_{i}, \mathbf{e}_{D i}\right)\right]+\underline{\delta}_{D}\left[D_{D}\left(\tau \mid t_{j}, \mathbf{p}_{i}, \mathbf{e}_{D i}\right)\right]}{2(b-a)}\right] / n S
$$

Similarly to Strategy 1 , the form of the approximations to $E_{E \cup A}\left[D_{D}(\tau) \mid \mathbf{a} \in \mathcal{A}_{1}(a, b)\right]$ and $p_{E \cup A}\left[D(\tau) \leq D \mid \mathbf{a} \in \mathcal{A}_{1}(a\right.$, $b)$ ] obtained in conjunction with Strategy 2 will, in general, depend on the properties of the procedure used to approximate the integral in Eq. (8.20) that defines $E_{t}\left[D_{D}\left(\tau \mid \mathbf{p}, \mathbf{e}_{D}, \lambda_{D}\right)\right]$.

The approximations to $E_{E \cup A}\left[D_{D}(\tau) \mid \mathbf{a} \in \mathcal{A}_{1}(a, b)\right]$ and $p_{E \cup A}\left[D_{D}(\tau) \leq D \mid \mathbf{a} \in \mathcal{A}_{1}(a, b)\right]$ with results obtained as part of Strategies 1 and 2 for the evaluation of $E_{E}\left\{E_{A}\left[D_{D}\left(\tau \mid \mathbf{a}, \mathbf{e}_{D}\right) \mid \mathbf{e}_{A}\right]\right\}$ are illustrated in Fig. 16 for the function $D_{D}\left(\tau \mid t, \mathbf{p}, \mathbf{e}_{D}\right)$ and associated definitions for $\mathbf{p}, \mathbf{e}_{A}$ and $\mathbf{e}_{D}$ introduced in conjunction with Eq. (8.3). As should be the case, the results obtained with Strategy 1 and Strategy 2 are very similar and also very similar to the results obtained with Strategy 3. Any differences that exist between the results obtained with the different strategies are resulting from approximation error rather than from inherent differences in the quantities being calculated.

Although interesting, the quantile curves in Figs. 15 and 16 do not correspond to results associated with the acceptance criteria in Quotes (YMRP4) and (YMRP5) because they do not include the effects of $\lambda_{D}$ and the uncertainty in $\lambda_{D}$ (e.g., see Quotes (NRC2), (YMRP3)). Specifically, the absence of $\lambda_{D}$ means that the likelihood of the disruptive event is not being included in the results summarized in Figs. 15 and 16. 


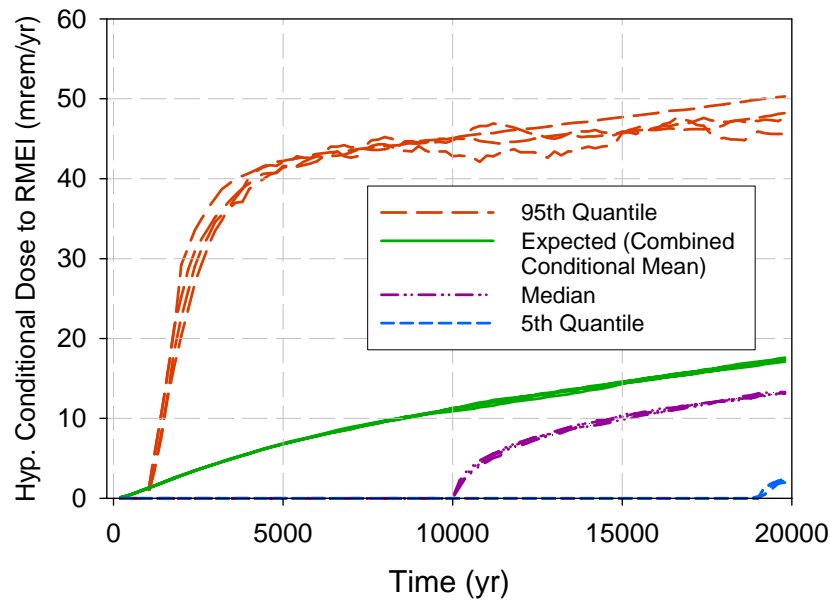

(a)

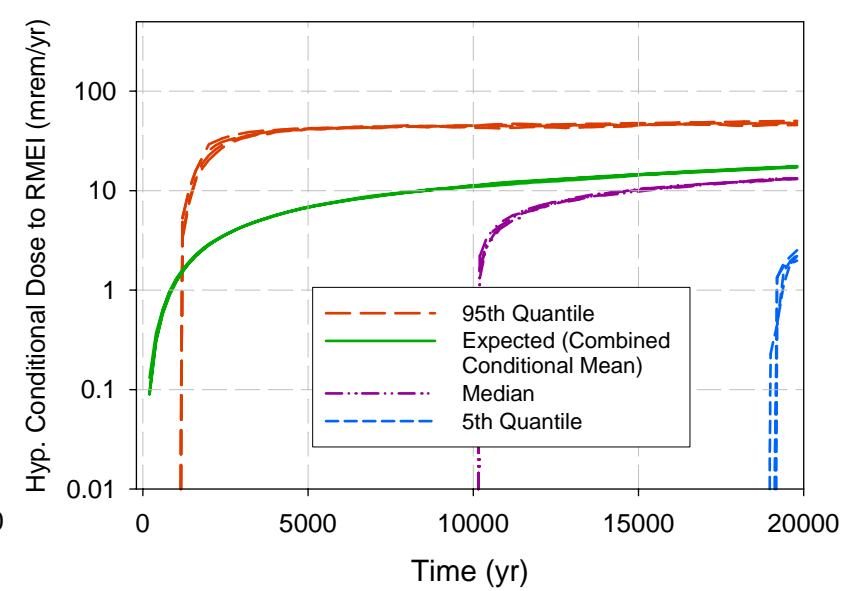

(b)

Fig. 16. Combined epistemic and aleatory uncertainty in hypothetical dose conditional on exactly one disruptive occurrence taking place in the time interval $[a, b]=[10,20,000 \mathrm{yr}]$ summarized as expected (combined conditional mean) dose $\hat{E}_{E \cup A}\left[D_{D}(\tau) \mid \mathbf{a} \in \mathcal{A}_{1}(a, b)\right]$ and associated quantiles (i.e., 0.05, 0.5, 0.95) for $D_{D}\left(\tau \mid t, \mathbf{p}, \mathbf{e}_{D}\right)$ conditional on $\mathbf{a}=[t, \mathbf{p}] \in \mathcal{A}_{1}(a, b)$ defined by $\hat{p}_{E \cup A}\left[D_{D}(\tau)<D \mid \mathbf{a} \in \mathcal{A}_{1}(a, b)\right]$ obtained with results from (i) Strategy 1 as indicated in Eqs. (9.15) and (9.16) with LHS of size 100, (ii) Strategy 2 as indicated in Eqs. (9.18) and (9.19) with LHS of size 100, and (iii) Strategy 3 as indicated in Eqs. (9.9) and (9.10) with LHSs of size 5000 and outcomes shown for both uniform and loguniform sampling on time (i.e., there are two sets of curves for Strategy 3): (a) Linear scale, and (b) Log scale. 


\section{Discussion}

As mandated in the Energy Policy Act of 1992, ${ }^{19}$ the EPA has promulgated public health and safety standards for radioactive material stored or disposed of in the YM repository ${ }^{20}$ and the NRC has developed licensing standards for the YM repository consistent with the EPA's public health and safety standards. ${ }^{21}$ In turn, the DOE is required to show that the YM repository meets the NRC standards. To facilitate the development and review of a licensing application for the YM repository, the NRC has also published a review plan. ${ }^{22}$

At the core of the standards and review plan indicated in the preceding paragraph is the requirement to carry out a PA for the YM repository that ultimately predicts expected dose to the RMEI. Further, there are many requirements that relate in various ways to the treatment and representation of the effects of uncertainty. Although the standards and review plan provide guidance with respect to the nature of the PA that DOE must carry out for the YM repository, this guidance is general and does not provide a complete technical (i.e., mathematical) description of what is desired. Thus, a necessary initial step in a PA for the YM repository is to convert the primarily nonquantitative and nonstructured requirements specified by the EPA and the NRC into a mathematical structure that can guide the conceptual and computational organization of the PA. Without the introduction of this structure, it is difficult to develop a PA for the YM repository that meets the EPA and NRC standards in a manner that is conceptually consistent, appropriately organized, and reasonably transparent. The purpose of this presentation is to describe how to conceptually organize and implement a PA for the YM repository in a manner that is consistent with the NRC and EPA standards. Fortunately, the general requirements on PA for the YM repository and the associated calculation of expected dose to the RMEI mandated by the EPA and NRC permit the design of a conceptually consistent analysis, which in turn provides the basis for a well organized computational analysis.

Near the beginning of this presentation, it is observed that, at an intuitive level, a PA can be viewed as an analysis carried out to answer three questions about a system and one question about the analysis itself (see Questions (Q1) - (Q4)): (i) "What can happen?”, (ii) "How likely is it to happen?”, (iii) "What are the consequences if it does happen?", and (iv) "What is the uncertainty in the answers to the first three questions?". Characterizing a PA in this manner provides a good indication of what a PA is supposed to provide. However, it does not provide the conceptual and mathematical structure that is needed to actually plan and implement a PA.

Actually answering the four preceding questions leads to an analysis (i.e., a PA) that involves three basic conceptual, and mathematically representable, entities: (i) a probabilistic characterization of what could occur in the system under study, (ii) a numerical procedure (i.e., a model) for predicting system behavior given a particular occurrence, and (iii) a probabilistic representation of the uncertainty in analysis inputs that have fixed but poorly known values. All three of the indicated entities are indicated, either specifically or by implication, at various places in the NRC and EPA standards for the YM repository. Formally, the first entity is a probability space characterizing aleatory uncertainty (i.e., uncertainty with respect to what might, or might not, occur in the future); the second entity corresponds to the mathematical models used to predict system behavior (i.e., very complicated functions of what could happen based on mathematical structures such as ordinary differential equations, partial differential equations, algorithmic procedures, ...); and the third entity is a probability space characterizing epistemic uncertainty (i.e., uncertainty with respect to the appropriate values of quantities that are assumed to have fixed values in the context of the particular analysis under consideration). Typically, the two indicated probability spaces are defined by assigning distributions to individual variables.

The preceding may sound intimidating but it corresponds to what must be defined to carry out a PA of the form indicated by the EPA and the NRC. In particular, the expected dose to the RMEI specified by the EPA and the NRC is defined by a very complex integral that involves all three entities as extensively discussed and illustrated in this presentation. Understanding the nature of these entities is essential to planning an evaluation of this integral

(i.e., calculating expected dose to the RMEI) and presenting the uncertainty associated with this calculation. Without a clear understanding, the calculation can be inappropriately implemented and the results of the calculation difficult, if not impossible, to interpret. 
Given a single set of definitions for the three indicated entities, this presentation has described three different computational strategies that lead to the same expected dose to the RMEI, with this expectation calculated over both aleatory uncertainty (i.e., the first entity) and epistemic uncertainty (i.e., the third entity). Although these strategies produce the same final result, they involve very different intermediate results and produce very different types of uncertainty information. Of the three presented strategies for the calculation of expected dose to the RMEI, the authors' preference is for the first strategy because it maintains a separation between aleatory and epistemic uncertainty. In turn, this allows a display of the uncertainty in expected dose that derives from epistemic uncertainty and also provides the basis for sensitivity analyses to determine the variables that dominate the uncertainty in expected dose. This separation of the effects of aleatory and epistemic uncertainty is consistent with the NRC's emphasis on the importance of an adequate treatment and display of the effects of uncertainty.

Probabilistic results involving both stationary and nonstationary Poisson processes are presented in Sect. 4. The results in Sects. 5 - 9 are presented for disruptive events whose potential occurrence is assumed to be characterized by a stationary Poisson process (i.e., for constant values for $\lambda_{D}, \lambda_{I}$ and $\lambda_{S}$ ). However, similar results hold for nonstationary Poisson processes and can be obtained with the corresponding results for Sect. 4 and appropriate modifications to the presented derivations.

This presentation has not considered the possible implications of the remand of the EPA standard for not requiring the consideration of dose to the RMEI to time of peak dose and the EPA's reformulated standard requiring the consideration of a median, rather than a mean, dose from 10,000 to $1,000,000$ yr. ${ }^{180-182}$ However, it is noted that the first computationally strategy provides a conceptually consistent approach to the calculation of both expected and median doses. 


\section{References}

1. $\quad$ Rechard, R.P. 2000. "Historical Background on Performance Assessment for the Waste Isolation Pilot Plant," Reliability Engineering and System Safety. Vol. 69, no. 1-3, pp. 5-46.

2. $\quad$ Carter, L.J. and T.H. Pigford. 1999. "The World's Growing Inventory of Civil Spent Fuel," Arms Control Today. Vol. 29, no. 1, pp. 8-14.

3. $\quad$ Ewing, R.C. 1999. "Less Geology in the Geological Disposal of Nuclear Waste," Science. Vol. 286, pp. 415416.

4. $\quad$ Ewing, R.C., M.S. Tierney, L.K. Konikow, and R.P. Rechard. 1999. "Performance Assessments for Nuclear Waste Repositories: A Dialogue on Their Values and Limitations," Risk Analysis. Vol. 19, no. 5, pp. 933958.

5. $\quad$ North, D.W. 1999. "A Perspective on Nuclear Waste," Risk Analysis. Vol. 19, no. 4, pp. 751-758.

6. Okrent, D. 1999. "On Intergenerational Equity and Its Clash with Intragenerational Equity and on the Need for Policies to Guide the Regulation of Disposal of Wastes and Other Activities Posing Very Long-Term Risks," Risk Analysis. Vol. 19, no. 5, pp. 877-901.

7. Rechard, R.P. 1999. "Historical Relationship Between Performance Assessment for Radioactive Waste Disposal and Other Types of Risk Assessment," Risk Analysis. Vol. 19, no. 5, pp. 763-807.

8. Ahearne, J.F. 1997. "Radioactive Waste: The Size of the Problem," Physics Today. Vol. 50, no. 6, pp. 24-29.

9. $\quad$ Crowley, K.D. 1997. "Nuclear Waste Disposal: The Technical Challenges," Physics Today. Vol. 50, no. 6, pp. 32-39.

10. Flynn, J., R.E. Kasperson, H. Kunreuther, and P. Slovic. 1997. "Overcoming Tunnel Vision: Redirecting the U.S. High-Level Nuclear Waste Program," Environment. Vol. 39, no. 3, pp. 6-11, 25-30.

11. Kastenburg, W.E. and L.J. Gratton. 1997. "Hazards of Managing and Disposing of Nuclear Waste," Physics Today. Vol. 50, no. 6, pp. 41-46.

12. McCombie, C. 1997. "Nuclear Waste Management Worldwide," Physics Today. Vol. 50, no. 6, pp. 56-62.

13. North, D.W. 1997. "Unresolved Problems of Radioactive Waste: Motivation for a New Paradigm," Physics Today. Vol. 50, no. 6, pp. 48-54.

14. U.S. DOE (U.S. Department of Energy). 2002. Final Environmental Impact Statement for a Geologic Repository for the Disposal of Spent Nuclear Fuel and High-Level Radioactive Waste at Yucca Mountain, Nye County, Nevada, DOE/EIS-0250F. Washington, D.C.: U.S. Department of Energy, Office of Civilian Radioactive Waste Management.

15. U.S. DOE (U.S. Department of Energy). 2002. Yucca Mountain Science and Engineering Report, Rev. 1, DOE/RW-05391. Washington, D.C.: U.S. Department of Energy, Office of Civilian Radioactive Waste Management.

16. CRWMS M\&O (Civilian Radioactive Waste Management System Management and Operating Contractor). 2000. Total System Performance Assessment for the Site Recommendation, TDR-WIS-PA-000001 REV 00. Las Vegas, NV: CRWMS M\&O. 
17. U.S. DOE (U.S. Department of Energy). 1998. Viability Assessment of a Repository at Yucca Mountain, DOE/RW-0508. Washington, D.C.: U.S. Department of Energy, Office of Civilian Radioactive Waste Management.

18. USGS (U.S. Geological Survey). 1999. Yucca Mountain as a Radioactive Waste Repository, Circular 1184. Denver, CO: USGS Information Services.

19. Public Law 102-486. 1992. Energy Policy Act of 1992.

20. U.S. EPA (U.S. Environmental Protection Agency). 2001. "40 CFR 197: Public Health and Environmental Protection Standards for Yucca Mountain, NV; Final Rule," Federal Register. Vol. 66, no. 114, pp. 3207432135.

21. U.S. NRC (U.S. Nuclear Regulatory Commission). 2001. "10 CFR Parts 2, 19, 20, etc.: Disposal of HighLevel Radioactive Wastes in a Proposed Geologic Repository at Yucca Mountain, Nevada; Final Rule," Federal Register. Vol. 66, no. 213, pp. 55732-55816.

22. U.S. NRC (U.S. Nuclear Regulatory Commission). 2003. Yucca Mountain Review Plan, Final Report, NUREG-1804, Rev. 2. Washington, D.C.: U.S. Nuclear Regulatory Commission.

23. Helton, J.C. 1997. "Uncertainty and Sensitivity Analysis in the Presence of Stochastic and Subjective Uncertainty," Journal of Statistical Computation and Simulation. Vol. 57, no. 1-4, pp. 3-76.

24. Helton, J.C. and D.E. Burmaster. 1996. "Guest Editorial: Treatment of Aleatory and Epistemic Uncertainty in Performance Assessments for Complex Systems," Reliability Engineering and System Safety. Vol. 54, no. 2-3, pp. 91-94.

25. Paté-Cornell, M.E. 1996. "Uncertainties in Risk Analysis: Six Levels of Treatment," Reliability Engineering and System Safety. Vol. 54, no. 2-3, pp. 95-111.

26. Winkler, R.L. 1996. "Uncertainty in Probabilistic Risk Assessment," Reliability Engineering and System Safety. Vol. 54, no. 2-3, pp. 127-132.

27. Hoffman, F.O. and J.S. Hammonds. 1994. "Propagation of Uncertainty in Risk Assessments: The Need to Distinguish Between Uncertainty Due to Lack of Knowledge and Uncertainty Due to Variability," Risk Analysis. Vol. 14, no. 5, pp. 707-712.

28. Helton, J.C. 1994. "Treatment of Uncertainty in Performance Assessments for Complex Systems," Risk Analysis. Vol. 14, no. 4, pp. 483-511.

29. Apostolakis, G. 1990. "The Concept of Probability in Safety Assessments of Technological Systems," Science. Vol. 250, no. 4986, pp. 1359-1364.

30. Haan, C.T. 1989. "Parametric Uncertainty in Hydrologic Modeling," Transactions of the ASAE. Vol. 32, no. 1, pp. 137-146.

31. Parry, G.W. and P.W. Winter. 1981. "Characterization and Evaluation of Uncertainty in Probabilistic Risk Analysis," Nuclear Safety. Vol. 22, no. 1, pp. 28-42.

32. Kaplan, S. and B.J. Garrick. 1981. "On the Quantitative Definition of Risk," Risk Analysis. Vol. 1, no. 1, pp. $11-27$.

33. Ghosh, S.T. and G.E. Apostolakis. 2006. "Extracting Risk Insights from Performance Assessments for HighLevel Radioactive Waste Repositories," Nuclear Technology. Vol. 153, no. 1, pp. 70-88. 
34. Garrick, B.J. 2005. "Activities of the Advisory Committee on Nuclear Waste and Perspectives on Selected Technical Issues," Practice Periodical of Hazardous, Toxic, and Radioactive Waste Management. Vol. 9, no. 1, pp. 33-44.

35. Carter, L.J. and T.H. Pigford. 2005. "Proof of Safety at Yucca Mountain," Science. Vol. 310, pp. 447-448.

36. Moeller, D.W. and M.T. Ryan. 2005. "Sensitivity Analyses of the Standards for the Proposed Yucca Mountain Repository--A Review, Evaluation, and Commentary," Health Physics. Vol. 88, no. 5, pp. 459-468.

37. Dyer, J.R. and M.T. Peters. 2004. "Progress in Permanent Geologic Disposal of Spent Nuclear Fuel and High-Level Radioactive Waste in the United States," Proceedings of the Institution of Mechanical Engineers -- Part A -- Power \& Energy. Vol. 218, no. 5, pp. 319-334.

38. Long, J.C.S. and R.C. Ewing. 2004. "Yucca Mountain: Earth-Science Issues at a Geologic Repository for High-Level Nuclear Waste," Annual Review of Earth and Planetary Science. Vol. 32, pp. 363-401.

39. Codell, R.B. 2004. "Alternative Igneous Source Term Model for Tephra Dispersal at the Yucca Mountain Repository," Nuclear Technology. Vol. 148, pp. 205-212.

40. Moeller, D.W. and M.T. Ryan. 2004. "Limitations on Upper Bound Dose to Adults Due to Intake of ${ }^{129}$ I in Drinking Water and a Total Diet--Implications Relative to the Proposed Yucca Mountain High Level Radioactive Waste Repository," Health Physics. Vol. 86, no. 6, pp. 586-589.

41. Mohanty, S. and R.B. Codell. 2004. "Independent Postclosure Performance Estimates of the Proposed Repository at Yucca Mountain," Nuclear Technology. Vol. 148, pp. 105-114.

42. Macfarlane, A. 2003. "Underlying Yucca Mountain: The Interplay of Geology and Policy in Nuclear Waste Disposal," Social Studies of Science. Vol. 33, no. 5, pp. 783-807.

43. Garrick, B.J. and V. Gilinsky. 2002. "Yucca Mountain: Pro \& Con," IEEE Spectrum. Vol. 39, no. 10, pp. 41-45.

44. Mohanty, S. and B. Sagar. 2002. "Importance of Transparency and Traceability in Building a Safety Case for High-Level Nuclear Waste Repositories," Risk Analysis. Vol. 22, no. 1, pp. 7-15.

45. Lu, Y. and S. Mohanty. 2001. "Sensitivity Analysis of a Complex, Proposed Geologic Waste Disposal System Using the Fourier Amplitude Sensitivity Test Method," Reliability Engineering and System Safety. Vol. 72, pp. 275-291.

46. Stepp, J.C., I. Wong, J. Whitney, R. Quittmeyer, N. Abrahamson, G. Toro, R. Youngs, K. Coppersmith, J. Savy, T. Sullivan, and Yucca Mountain PSHA Project Members. 2001. "Probabilistic Seismic Hazard Analyses for Ground Motions and Fault Displacement at Yucca Mountain, Nevada," Earthquake Spectra. Vol. 17, no. 1, pp. 113-151.

47. Mohanty, S. and Y-T. Wu. 2001. "CDF Sensitivity Analysis Techniques for Ranking Influential Parameters in the Performance Assessment of the Proposed High-Level Waste Repository at Yucca Mountain, Nevada, USA," Reliability Engineering and System Safety. Vol. 73, pp. 167-176.

48. Cragnolino, G.A., S. Mohanty, D.S. Dunn, N. Sridhar, and T.M. Ahn. 2000. "An Approach to the Assessment of High-Level Radioactive Waste Containment. I: Waste Package Degradation," Nuclear Engineering and Design. Vol. 201, pp. 289-306. 
49. Mohanty, S., R.B. Codell, T.M. Ahn, and G.A. Cragnolino. 2000. "An Approach to the Assessment of HighLevel Radioactive Waste Containment. II: Radionuclide Releases From an Engineered Barrier System," Nuclear Engineering and Design. Vol. 201, pp. 307-325.

50. Jarzemba, M.S. 1997. "Stochastic Radionuclide Distributions After a Basaltic Eruption for Performance Assessments of Yucca Mountain," Nuclear Technology. Vol. 118, pp. 132-141.

51. Garrick, B.J. and S. Kaplan. 1994. Quantitative Performance Assessment of Nuclear Waste Repositories, Prepared for U.S. Nuclear Waste Technical Review Board. Irvine, CA: Pickard, Lowe and Garrick, Inc.

52. Helton, J.C., D.R. Anderson, G. Basabilvazo, H.-N. Jow, and M.G. Marietta. 2000. "Conceptual Structure of the 1996 Performance Assessment for the Waste Isolation Pilot Plant," Reliability Engineering and System Safety. Vol. 69, no. 1-3, pp. 151-165.

53. Helton, J.C. 2003. "Mathematical and Numerical Approaches in Performance Assessment for Radioactive Waste Disposal: Dealing with Uncertainty," Modelling Radioactivity in the Environment. Eds. E.M. Scott. New York, NY: Elsevier Science. 353-390.

54. NRC (National Research Council). 1995. Technical Bases for Yucca Mountain Standards, Washington, DC: National Academy Press.

55. U.S. NRC (U.S. Nuclear Regulatory Commission). 2002. "10 CFR Part 63: Specification of a Probability for Unlikely Features, Events and Processes," Federal Register. Vol. 67, no. 195, pp. 62628-62634.

56. U.S. NRC (U.S. Nuclear Regulatory Commission). 1975. Reactor Safety Study-An Assessment of Accident Risks in U.S. Commercial Nuclear Power Plants, WASH-1400 (NUREG-75/014). Washington, DC: U.S. Nuclear Regulatory Commission.

57. PLG (Pickard Lowe and Garrick, I., Westinghouse Electric Corporation, and Fauske \& Associates, Inc.). 1983. Seabrook Station Probabilistic Safety Assessment. Newport Beach: Prepared for Public Service Company of New Hampshire and Yankee Atomic Electric Company. Irvine: Pickard, Lowe and Garrick, Inc. (Available from the NTIS as PB84-186949 and PB84-186931.).

58. PLG (Pickard Lowe and Garrick, I., Westinghouse Electric Corporation, and Fauske \& Associates, Inc.). 1982. Indian Point Probabilistic Safety Study, Prepared for the Power Authority of the State of New York and Consolidated Edison Company of New York, Inc. Irvine, CA: Pickard, Lowe and Garrick, Inc.

59. U.S. NRC (U.S. Nuclear Regulatory Commission). 1990-1991. Severe Accident Risks: An Assessment for Five U.S. Nuclear Power Plants, NUREG-1150, Vols. 1-3. Washington, DC: U.S. Nuclear Regulatory Commission, Office of Nuclear Regulatory Research, Division of Systems Research.

60. Breeding, R.J., J.C. Helton, E.D. Gorham, and F.T. Harper. 1992. "Summary Description of the Methods Used in the Probabilistic Risk Assessments for NUREG-1150," Nuclear Engineering and Design. Vol. 135, no. 1, pp. 1-27.

61. U.S. DOE (U.S. Department of Energy). 1996. Title 40 CFR Part 191 Compliance Certification Application for the Waste Isolation Pilot Plant, DOE/CAO-1996-2184, Vols. I-XXI. Carlsbad, NM: U.S. Department of Energy, Carlsbad Area Office, Waste Isolation Pilot Plant.

62. Helton, J.C. and M.G. Marietta. 2000. "Special Issue: The 1996 Performance Assessment for the Waste Isolation Pilot Plant," Reliability Engineering and System Safety. Vol. 69, no. 1-3, pp. 1-451.

63. Feller, W. 1971. An Introduction to Probability Theory and Its Applications. Vol. 2, 2nd ed. New York, NY: John Wiley \& Sons. 
64. Hsu, M., M. Bhatt, R. Adolphs, D. Tranel, and C.F. Camerer. 2005. "Neural Systems Responding to Degrees of Uncertainty in Human Decision-Making," Science. Vol. 310, no. 5754, pp. 1680-1683.

65. Garthwaite, P.H., J.B. Kadane, and A. O'Hagan. 2005. "Statistical Methods for Eliciting Probability Distributions," Journal of the American Statistical Association. Vol. 100, no. 470, pp. 680-700.

66. Cooke, R.M. and L.H.J. Goossens. 2004. "Expert Judgement Elicitation for Risk Assessment of Critical Infrastructures," Journal of Risk Research. Vol. 7, no. 6, pp. 643-656.

67. Ayyub, B.M. 2001. Elicitation of Expert Opinions for Uncertainty and Risks, Boca Raton, FL: CRC Press.

68. McKay, M. and M. Meyer. 2000. "Critique of and Limitations on the use of Expert Judgements in Accident Consequence Uncertainty Analysis," Radiation Protection Dosimetry. Vol. 90, no. 3, pp. 325-330.

69. Budnitz, R.J., G. Apostolakis, D.M. Boore, L.S. Cluff, K.J. Coppersmith, C.A. Cornell, and P.A. Morris. 1998. "Use of Technical Expert Panels: Applications to Probabilistic Seismic Hazard Analysis," Risk Analysis. Vol. 18, no. 4, pp. 463-469.

70. Evans, J.S., G.M. Gray, R.L. Sielken Jr., A.E. Smith, C. Valdez-Flores, and J.D. Graham. 1994. "Use of Probabilistic Expert Judgement in Uncertainty Analysis of Carcinogenic Potency," Regulatory Toxicology and Pharmacology. Vol. 20, no. 1, pt. 1, pp. 15-36.

71. Chhibber, S., G. Apostolakis, and D. Okrent. 1992. "A Taxonomy of Issues Related to the Use of Expert Judgments in Probabilistic Safety Studies," Reliability Engineering and System Safety. Vol. 38, no. 1-2, pp. 27-45.

72. Thorne, M.C. and M.M.R. Williams. 1992. "A Review of Expert Judgement Techniques with Reference to Nuclear Safety," Progress in Nuclear Safety. Vol. 27, no. 2-3, pp. 83-254.

73. Cooke, R.M. 1991. Experts in Uncertainty: Opinion and Subjective Probability in Science. Oxford; New York: Oxford University Press.

74. Keeney, R.L. and D.V. Winterfeldt. 1991. "Eliciting Probabilities from Experts in Complex Technical Problems," IEEE Transactions on Engineering Management. Vol. 38, no. 3, pp. 191-201.

75. Meyer, M.A. and J.M. Booker. 1991. Eliciting and Analyzing Expert Judgment: A Practical Guide. New York, NY: Academic Press.

76. Hora, S.C. and R.L. Iman. 1989. "Expert Opinion in Risk Analysis: The NUREG-1150 Methodology," Nuclear Science and Engineering. Vol. 102, no. 4, pp. 323-331.

77. Ortiz, N.R., T.A. Wheeler, R.J. Breeding, S. Hora, M.A. Meyer, and R.L. Keeney. 1991. "Use of Expert Judgment in NUREG-1150," Nuclear Engineering and Design. Vol. 126, no. 3, pp. 313-331.

78. Harper, F.T., R.J. Breeding, T.D. Brown, J.J. Gregory, A.C. Payne, E.D. Gorham, and C.N. Amos. 1990. Evaluation of Severe Accident Risks: Quantification of Major Input Parameters, Expert Opinion Elicitation on In-Vessel Issues, NUREG/CR-4551, SAND86-1309, Vol. 2, Part 1, Rev. 1. Albuquerque, NM: Sandia National Laboratories.

79. Harper, F.T., A.C. Payne, R.J. Breeding, E.D. Gorham, T.D. Brown, G.S. Rightley, J.J. Gregory, W. Murfin, and C.N. Amos. 1991. Evaluation of Severe Accident Risks: Quantification of Major Input Parameters, Experts' Determination of Containment Loads and Molten Core-Concrete Issues, NUREG/CR-4551, SAND861309, Vol. 2, Part 2, Rev. 1. Albuquerque, NM: Sandia National Laboratories. 
80. Breeding, R.J., F.T. Harper, T.D. Brown, J.J. Gregory, A.C. Payne, E.D. Gorham, W. Murfin, and C.N. Amos. 1992. Evaluation of Severe Accident Risks: Quantification of Major Input Parameters, Experts' Determination of Structural Response Issues, NUREG/CR-4551, SAND86-1309, Vol. 2, Part 3, Rev. 1. Albuquerque, NM: Sandia National Laboratories.

81. Harper, F.T., R.J. Breeding, T.D. Brown, J.J. Gregory, H.-N. Jow, A.C. Payne, E.D. Gorham, C.N. Amos, J.C. Helton, and G. Boyd. 1992. Evaluation of Severe Accident Risks: Quantification of Major Input Parameters, Experts' Determination of Source Term Issues, NUREG/CR-4551, SAND86-1309, Vol. 2, Part 4, Rev. 1. Albuquerque, NM: Sandia National Laboratories.

82. Wheeler, T.A., S.C. Hora, W.R. Cramond, and S.D. Unwin. 1989. Analysis of Core Damage Frequency: Expert Judgment Elicitation, NUREG/CR-4550, SAND86-2084, Vol. 2, Rev. 1. Albuquerque, NM: Sandia National Laboratories.

83. Harper, F.T., L.H.J. Goossens, R.M. Cooke, S.C. Hora, M.L. Young, J. Päsler-Sauer, L.A. Miller, B. Kraan, C.H. Lui, M.D. McKay, J.C. Helton, and J.A. Jones. 1995. Probabilistic Accident Consequence Uncertainty Analysis: Dispersion and Deposition Uncertainty Assessment, NUREG/CR-6244, EUR 15855EN, SAND941453, Vols. 1-3. Washington, D.C.: U.S. Nuclear Regulatory Commission.

84. Goossens, L.H.J., J. Boardman, B.C.P. Kraan, R.M. Cooke, J.A. Jones, F.T. Harper, M.L. Young, and S.C. Hora. 1997. Probabilistic Accident Consequence Uncertainty Analysis: Uncertainty Assessment for Deposited Material and External Doses, NUREG/CR-6526, EUR 16772, SAND97-2323, Vols. 1 \& 2. Washington, D.C.: U.S. Nuclear Regulatory Commission.

85. Haskin, F.E., F.T. Harper, L.H.J. Goossens, B.C.P. Kraan, and J.B. Grupa. 1997. Probabilistic Accident Consequence Uncertainty Analysis: Early Health Effects Uncertainty Assessment, NUREG/CR-6545, EUR 16775, SAND97-2689, Vols. 1 \& 2. Washington, D.C.: U.S. Nuclear Regulatory Commission.

86. Little, M.P., C.R. Muirhead, L.H.J. Goossens, B.C.P. Kraan, R.M. Cooke, F.T. Harper, and S.C. Hora. 1997. Probabilistic Accident Consequence Uncertainty Analysis: Late Health Effects Uncertainty Assessment, NUREG/CR-6555, EUR 16774, SAND97-2322, Vols. 1 \& 2. Washington, D.C.: U.S. Nuclear Regulatory Commission.

87. Goossens, L.H.J., J.D. Harrison, B.C.P. Kraan, R.M. Cooke, F.T. Harper, and S.C. Hora. 1998. Probabilistic Accident Consequence Uncertainty Analysis: Uncertainty Assessment for Internal Dosimetry, NUREG/CR6571, EUR 16773, SAND98-0119, Vols. 1 \& 2. Washington, D.C.: U.S. Nuclear Regulatory Commission.

88. Klir, G.J. 2005. Uncertainty and Information: Foundations of Generalized Information Theory. Hoboken, NJ: Wiley.

89. Helton, J.C., J.D. Johnson, and W.L. Oberkampf. 2004. "An Exploration of Alternative Approaches to the Representation of Uncertainty in Model Predictions," Reliability Engineering and System Safety. Vol. 85, no. 1-3, pp. 39-71.

90. Klir, G.J. 2004. "Generalized Information Theory: Aims, Results, and Open Problems," Reliability Engineering and System Safety. Vol. 85, no. 1-3, pp. 21-38.

91. Ross, T.J. 2004. Fuzzy Logic with Engineering Applications. 2nd ed. New York, NY: Wiley.

92. Halpern, J.Y. 2003. Reasoning about Uncertainty. Cambridge, MA: MIT Press.

93. Ross, T.J., J.M. Booker, and W.J. Parkinson (eds.). 2002. Fuzzy Logic and Probability Applications: Bridging the Gap. Philadelphia, PA: Society for Industrial and Applied Mathematics. 
94. Jaulin, L., M. Kieffer, O. Didrit, and E. Walter. 2001. Applied Interval Analysis. New York, NY: SpringerVerlag.

95. Wolkenhauer, O. 2001. Data Engineering: Fuzzy Mathematics in Systems Theory and Data Analysis. New York, NY: Wiley.

96. Klir, G.J. and M.J. Wierman. 1999. Uncertainty-Based Information, New York, NY: Physica-Verlag.

97. Yager, R.R., J. Kacprzyk, and M. Fedrizzi (eds). 1994. Advances in the Dempster-Shafer Theory of Evidence. New York, NY: Wiley.

98. Diaz, N.J. 2003. "Realism and Conservatism, Remarks by Chairman Diaz at the 2003 Nuclear Safety Research Conference, October 20, 2003," NRC News. No. S-03-023. Washington, D.C.: U.S. Nuclear Regulatory Commission.

99. Paté-Cornell, E. 2002. "Risk and Uncertainty Analysis in Government Safety Decisions," Risk Analysis. Vol. 22, no. 3, pp. 633-646.

100. Caruso, M.A., M.C. Cheok, M.A. Cunningham, G.M. Holahan, T.L. King, G.W. Parry, A.M. Ramey-Smith, M.P. Rubin, and A.C. Thadani. 1999. "An Approach for Using Risk Assessment in Risk-Informed Decisions on Plant-Specific Changes to the Licensing Basis," Reliability Engineering and System Safety. Vol. 63, pp. 231-242.

101. Sielken, R.L., Jr., R.S. Bretzlaff, and D.E. Stevenson. 1995. "Challenges to Default Assumptions Stimulate Comprehensive Realism as a New Tier in Quantitative Cancer Risk Assessment," Regulatory Toxicology and Pharmacology. Vol. 21, pp. 270-280.

102. Nichols, A.L. and R.J. Zeckhauser. 1988. "The Perils of Prudence: How Conservative Risk Assessments Distort Regulation," Regulatory Toxicology and Pharmacology. Vol. 8, pp. 61-75.

103. MacKinnon, R.J., G. Freeze, and H.-N. Jow. 1997. Summary of EPA-Mandated Performance Assessment Verification Test (Replicate 1) and Comparison with the Compliance Certification Application Calculations, Technical Data Package (Sandia WIPP Central Files WPO \#46674). Albuquerque, NM: Sandia National Laboratories.

104. Mosleh, A., N. Siu, C. Smidts, and C. Liu. 1994. "Proceedings of Workshop I in Advanced Topics in Risk and Reliability Analysis, Model Uncertainty: Its Characterization and Quantification," NUREG/CP-0138. Washington, D.C.: U.S. Nuclear Regulatory Commission.

105. Hacking, I. 1975. The Emergence of Probability: A Philosophical Study of Early Ideas About Probability, Induction and Statistical Inference. London; New York: Cambridge University Press.

106. Shafer, G. 1978. "Non-Additive Probabilities in Work of Bernoulli and Lambert," Archive for History of Exact Sciences. Vol. 19, no. 4, pp. 309-370.

107. Bernstein, P.L. 1996. Against the Gods: The Remarkable Story of Risk. New York: John Wiley \& Sons.

108. Paté-Cornell, M.E. 1986. "Probability and Uncertainty in Nuclear Safety Decisions," Nuclear Engineering and Design. Vol. 93, no. 2-3, pp. 319-327.

109. Apostolakis, G.E. 1989. "Uncertainty in Probabilistic Risk Assessment," Nuclear Engineering and Design. Vol. 115, pp. 173-179. 
110. Kaplan, S. 1993. "Formalisms for Handling Phenomenological Uncertainties: The Concepts of Probability, Frequency, Variability, and Probability of Frequency," Nuclear Technology. Vol. 102, no. 1, pp. 137-142.

111. Connor, C.B. and B.E. Hill. 1995. "Three Nonhomogeneous Poisson Models for the Probability of Basaltic Volcanism: Application to the Yucca Mountain Region, Nevada," Journal of Geophysical Research. Vol. 100, no. B6, pp. 10, 107-110, 125.

112. Ross, S.M. 1993. Introduction to Probability Models. 5th ed. New York, NY: Academic Press.

113. Cramér, H. and H. Leadbetter. 1966. Stationary and Related Stochastic Processes. New York, NY: Wiley.

114. Haight, F.A. 1967. Handbook of the Poisson Distribution. New York: John Wiley \& Sons.

115. Ross, S.M. 1983. Stochastic Processes. New York, NY: Wiley.

116. Helton, J.C. 1993. "Drilling Intrusion Probabilities for Use in Performance Assessment for Radioactive Waste Disposal," Reliability Engineering and System Safety. Vol. 40, no. 3, pp. 259-275.

117. Parzen, E. 1960. Modern Probability Theory and Its Applications. New York, NY: John Wiley \& Sons.

118. Evans, M. and T. Swartz. 2000. Approximating Integrals via Monte Carlo and Deterministic Methods. Oxford, New York: Oxford University Press.

119. McKay, M.D., R.J. Beckman, and W.J. Conover. 1979. "A Comparison of Three Methods for Selecting Values of Input Variables in the Analysis of Output from a Computer Code," Technometrics. Vol. 21, no. 2, pp. 239-245.

120. Helton, J.C. and F.J. Davis. 2003. "Latin Hypercube Sampling and the Propagation of Uncertainty in Analyses of Complex Systems," Reliability Engineering and System Safety. Vol. 81, no. 1, pp. 23-69.

121. Iman, R.L. and W.J. Conover. 1980. "Small Sample Sensitivity Analysis Techniques for Computer Models, with an Application to Risk Assessment," Communications in Statistics: Theory and Methods. Vol. A9, no. 17, pp. 1749-1842.

122. Iman, R.L., J.C. Helton, and J.E. Campbell. 1981. "An Approach to Sensitivity Analysis of Computer Models, Part 1. Introduction, Input Variable Selection and Preliminary Variable Assessment," Journal of Quality Technology. Vol. 13, no. 3, pp. 174-183.

123. Iman, R.L., J.C. Helton, and J.E. Campbell. 1981. "An Approach to Sensitivity Analysis of Computer Models, Part 2. Ranking of Input Variables, Response Surface Validation, Distribution Effect and Technique Synopsis," Journal of Quality Technology. Vol. 13, no. 4, pp. 232-240.

124. Iman, R.L. 1992. "Uncertainty and Sensitivity Analysis for Computer Modeling Applications," Reliability Technology - 1992, The Winter Annual Meeting of the American Society of Mechanical Engineers, Anaheim, California, November 8-13, 1992. Eds. T.A. Cruse. Vol. 28, pp. 153-168. New York, NY: American Society of Mechanical Engineers, Aerospace Division.

125. Helton, J.C. 1993. "Uncertainty and Sensitivity Analysis Techniques for Use in Performance Assessment for Radioactive Waste Disposal," Reliability Engineering and System Safety. Vol. 42, no. 2-3, pp. 327-367.

126. Kleijnen, J.P.C. and J.C. Helton. 1999. "Statistical Analyses of Scatterplots to Identify Important Factors in Large-Scale Simulations, 1: Review and Comparison of Techniques," Reliability Engineering and System Safety. Vol. 65, no. 2, pp. 147-185. 
127. Helton, J.C. and F.J. Davis. 2000. "Sampling-Based Methods," Sensitivity Analysis. Ed. A. Saltelli, K. Chan, and E.M. Scott. New York, NY: Wiley. pp. 101-153.

128. Helton, J.C. and F.J. Davis. 2002. "Illustration of Sampling-Based Methods for Uncertainty and Sensitivity Analysis," Risk Analysis. Vol. 22, no. 3, pp. 591-622.

129. Helton, J.C., J.D. Johnson, C.J. Sallaberry, and C.B. Storlie. 2006. "Survey of Sampling-Based Methods for Uncertainty and Sensitivity Analysis," Reliability Engineering and System Safety. Vol. 91, no. 10-11, pp. 1175-1209.

130. Campbell, J.E., R.T. Dillon, M.S. Tierney, H.T. Davis, P.E. McGrath, F.J. Pearson, H.R. Shaw, J.C. Helton, and F.A. Donath. 1978. Risk Methodology for Geologic Disposal of Radioactive Waste: Interim Report, SAND78-0029, NUREG/CR-0458. Albuquerque, NM: Sandia National Laboratories.

131. Iman, R.L., J.C. Helton, and J.E. Campbell. 1978. Risk Methodology for Geologic Disposal of Radioactive Waste: Sensitivity Analysis Techniques, SAND78-0912, NUREG/CR-0390. Albuquerque, NM: Sandia National Laboratories.

132. Iman, R.L., J.M. Davenport, and D.K. Ziegler. 1980. Latin Hypercube Sampling (Program User's Guide), SAND79-1473. Albuquerque, NM: Sandia National Laboratories.

133. Iman, R.L. and W.J. Conover. 1980. Risk Methodology for Geologic Disposal of Radioactive Waste - A Distribution-Free Approach to Inducing Rank Correlation Among Input Variables for Simulation Studies, SAND80-0157, NUREG-CR-1262. Albuquerque, NM: Sandia National Laboratories.

134. Iman, R.L., W.J. Conover, and J.E. Campbell. 1980. Risk Methodology for Geologic Disposal of Radioactive Waste: Small Sample Sensitivity Analysis Techniques for Computer Models, with an Application to Risk Assessment, SAND80-0020, NUREG-CR-1397. Albuquerque, NM: Sandia National Laboratories.

135. Iman, R.L. and J.M. Davenport. 1980. Rank Correlation Plots for Use with Correlated Input Variables in Simulation Studies, SAND80-1903. Albuquerque, NM: Sandia National Laboratories.

136. Helton, J.C. and R.L. Iman. 1980. Risk Methodology for Geologic Disposal of Radioactive Waste: Sensitivity Analysis of the Environmental Transport Model, NUREG/CR-1636, SAND79-1393. Albuquerque, NM: Sandia National Laboratories.

137. Campbell, J.E., R.L. Iman, and M. Reeves. 1980. Risk Methodology for Geologic Disposal of Radioactive Waste: Transport Model Sensitivity Analysis, NUREG/CR-1377, SAND80-0644. Albuquerque, NM: Sandia National Laboratories.

138. Iman, R.L., J.M. Davenport, E.L. Frost, and M.J. Shortencarier. 1980. Stepwise Regression with PRESS and Rank Regression (Program User's Guide), SAND79-1472. Albuquerque, NM: Sandia National Laboratories.

139. Iman, R.L. and W.J. Conover. 1982. Sensitivity Analysis Techniques, Self-Teaching Curriculum, NUREG/CR-2350, SAND81-1978. Albuquerque, NM: Sandia National Laboratories.

140. Cranwell, R.M., J.E. Campbell, J.C. Helton, R.L. Iman, D.E. Longsine, N.R. Ortiz, G.E. Runkle, and M.J. Shortencarier. 1987. Risk Methodology for Geologic Disposal of Radioactive Waste: Final Report, SAND81-2573, NUREG/CR-2452. Albuquerque, NM: Sandia National Laboratories.

141. Lewis, H.W., R.J. Budnitz, H.J.C. Kouts, W.B. Loewenstein, W.D. Rowe, F. von Hippel, and F. Zachariasen. 1978. Risk Assessment Review Group Report to the U.S. Nuclear Regulatory Commission, NUREG/CR0400. Washington, D.C.: U.S. Nuclear Regulatory Commission. 
142. Sprung, J.L., D.C. Aldrich, D.J. Alpert, M.A. Cunningham, and G.G. Weigand. 1983. "Overview of the MELCOR Risk Code Development Program," Proceedings of the International Meeting on Light Water Reactor Severe Accident Evaluation. Cambridge, MA, August 28-September 1, 1983. Boston, MA: Stone and Webster Engineering Corporation: TS-10.1-1 to TS-10.1-8.

143. Iman, R.L. and M.J. Shortencarier. 1984. A FORTRAN 77 Program and User's Guide for the Generation of Latin Hypercube and Random Samples for Use with Computer Models, NUREG/CR-3624, SAND83-2365. Albuquerque, NM: Sandia National Laboratories.

144. Alpert, D.J., R.L. Iman, J.C. Helton, and J.D. Johnson. 1985. A Demonstration Uncertainty/Sensitivity Analysis Using the Health and Economic Consequence Model CRAC2, NUREG/CR-4199, SAND84-1824. Albuquerque: Sandia National Laboratories.

145. Iman, R.L. and J.C. Helton. 1985. A Comparison of Uncertainty and Sensitivity Analysis Techniques for Computer Models, NUREG/CR-3904, SAND84-1461. Albuquerque, NM: Sandia National Laboratories.

146. Iman, R.L., M.J. Shortencarier, and J.D. Johnson. 1985. A FORTRAN 77 Program and User's Guide for the Calculation of Partial Correlation and Standardized Regression Coefficients, NUREG/CR-4122, SAND850044. Albuquerque, NM: Sandia National Laboratories.

147. Helton, J.C., R.L. Iman, J.D. Johnson, and C.D. Leigh. 1985. Uncertainty and Sensitivity Analysis of a Model for Multicomponent Aerosol Dynamics, NUREG/CR-4342, SAND84-1307. Albuquerque, New Mexico: Sandia National Laboratories.

148. Helton, J.C., R.L. Iman, J.D. Johnson, and C.D. Leigh. 1986. Uncertainty and Sensitivity Analysis of a Dry Containment Test Problem for the MAEROS Aerosol Model, NUREG/CR-4487, SAND85-2795. Albuquerque, New Mexico: Sandia National Laboratories.

149. Helton, J.C., R.L. Iman, J.D. Johnson, and C.D. Leigh. 1986. Uncertainty and Sensitivity Analysis of an Upper Plenum Test Problem for the MAEROS Aerosol Model, NUREG/CR-4460, SAND85-2196. Albuquerque, New Mexico: Sandia National Laboratories.

150. Breeding, R.J., J.C. Helton, W.B. Murfin, L.N. Smith, J.D. Johnson, H.-N. Jow, and A.W. Shiver. 1992. "The NUREG-1150 Probabilistic Risk Assessment for the Surry Nuclear Power Station," Nuclear Engineering and Design. Vol. 135, no. 1, pp. 29-59.

151. Payne, A.C., Jr., R.J. Breeding, J.C. Helton, L.N. Smith, J.D. Johnson, H.-N. Jow, and A.W. Shiver. 1992. "The NUREG-1150 Probabilistic Risk Assessment for the Peach Bottom Atomic Power Station," Nuclear Engineering and Design. Vol. 135, no. 1, pp. 61-94.

152. Gregory, J.J., R.J. Breeding, J.C. Helton, W.B. Murfin, S.J. Higgins, and A.W. Shiver. 1992. "The NUREG1150 Probabilistic Risk Assessment for the Sequoyah Nuclear Plant," Nuclear Engineering and Design. Vol. 135, no. 1, pp. 92-115.

153. Brown, T.D., R.J. Breeding, J.C. Helton, H.-N. Jow, S.J. Higgins, and A.W. Shiver. 1992. "The NUREG1150 Probabilistic Risk Assessment for the Grand Gulf Nuclear Station," Nuclear Engineering and Design. Vol. 135, no. 1, pp. 117-137.

154. Helton, J.C. and R.J. Breeding. 1993. "Calculation of Reactor Accident Safety Goals," Reliability Engineering and System Safety. Vol. 39, no. 2, pp. 129-158.

155. Payne, A.C., Jr. 1992. Analysis of the LaSalle Unit 2 Nuclear Power Plant: Risk Methods Integration and Evaluation Program (RMIEP). Summary, NUREG/CR-4832; SAND92-0537, Vol. 1. Albuquerque, NM: Sandia National Laboratories. 
156. Helton, J.C., J.A. Rollstin, J.L. Sprung, and J.D. Johnson. 1990. An Exploratory Sensitivity Study with the MACCS Reactor Accident Consequence Model, SAND88-1465. Albuquerque, NM: Sandia National Laboratories.

157. Helton, J.C., J.D. Johnson, M.D. McKay, A.W. Shiver, and J.L. Sprung. 1995. Uncertainty and Sensitivity Analysis of Early Exposure Results with the MACCS Reactor Accident Consequence Model, NUREG/CR6135, SAND93-2371. Albuquerque, NM: Sandia National Laboratories.

158. Helton, J.C., J.D. Johnson, J.A. Rollstin, A.W. Shiver, and J.L. Sprung. 1995. Uncertainty and Sensitivity Analysis of Food Pathway Results with the MACCS Reactor Accident Consequence Model, NUREG/CR6136, SAND93-2372. Albuquerque, NM: Sandia National Laboratories.

159. Helton, J.C., J.D. Johnson, J.A. Rollstin, A.W. Shiver, and J.L. Sprung. 1995. Uncertainty and Sensitivity Analysis of Chronic Exposure Results with the MACCS Reactor Accident Consequence Model, NUREG/CR6134, SAND93-2370. Albuquerque, NM: Sandia National Laboratories.

160. Roache, P.J. 1998. Verification and Validation in Computational Science and Engineering. Albuquerque, NM: Hermosa Publishers.

161. Oberkampf, W.L., S.M. DeLand, B.M. Rutherford, K.V. Diegert, and K.F. Alvin. 2002. "Error and Uncertainty in Modeling and Simulation," Reliability Engineering and System Safety. Vol. 75, no. 3, pp. 333-357.

162. Babuska, I. and J.T. Oden. 2004. "Verification and Validation in Computational Engineering and Science: Basic Concepts," Computer Methods in Applied Mechanics and Engineering. Vol. 193, no. 36-38, pp. 40574066.

163. Walpole, R.E. and R.H. Myers. 1985. Probability and Statistics for Engineers and Scientists. 3rd ed. New York, NY: Macmillan.

164. Iman, R.L. 1981. "Statistical Methods for Including Uncertainties Associated With the Geologic Isolation of Radioactive Waste Which Allow for a Comparison With Licensing Criteria," Proceedings of the Symposium on Uncertainties Associated with the Regulation of the Geologic Disposal of High-Level Radioactive Waste. NUREG/CP-0022; CONF-810372. Eds. D.C. Kocher. Gatlinburg, TN: Washington, DC: US Nuclear Regulatory Commission, Directorate of Technical Information and Document Control. 145-157.

165. Helton, J.C., M.-A. Martell, and M.S. Tierney. 2000. "Characterization of Subjective Uncertainty in the 1996 Performance Assessment for the Waste Isolation Pilot Plant," Reliability Engineering and System Safety. Vol. 69, no. 1-3, pp. 191-204.

166. Trucano, T.G., L.P. Swiler, T. Igusa, W.L. Oberkampf, and M. Pilch. 2006. "Calibration, Validation, and Sensitivity Analysis: What's What," Reliability Engineering and System Safety. Vol. 91, no. 10-11, pp. 13311357.

167. Oberkampf, W.L., T.G. Trucano, and C. Hirsch. 2004. "Verification, Validation, and Predictive Capability in Computational Engineering and Physics," Applied Mechanics Review. Vol. 57, no. 5, pp. 345-384.

168. Roache, P.J. 2004. "Building PDE Codes to be Verifiable and Validatable," Computing in Science \& Engineering. Vol. 6, no. 5, pp. 30-38.

169. Knupp, P. and K. Salari. 2002. Verification of Computer Codes in Computational Science and Engineering. Boca Raton, FL: Chapman and Hall/CRC.

170. Oberkampf, W.L. and T.G. Trucano. 2002. "Verification and Validation in Computational Fluid Dynamics," Progress in Aerospace Sciences. Vol. 38, no. 3, pp. 209-272. 
171. American Institute of Aeronautics and Astronautics. 1998. AIAA Guide for the Verification and Validation of Computational Fluid Dynamics Simulations, AIAA G-077-1998. Reston, VA: American Institute of Aeronautics and Astronautics.

172. Roache, P.J. 1997. "Quantification of Uncertainty in Computational Fluid Dynamics," Annual Review of Fluid Mechanics. Vol. 29, pp. 123-160.

173. Nicola, V.F., P. Shahabuddin, and M.K. Nakayama. 2001. "Techniques for Fast Simulation of Models of Highly Dependable Systems," IEEE Transactions on Reliability. Vol. 50, no. 3, pp. 246-264.

174. Owen, A. and Y. Zhou. 2000. "Safe and Effective Importance Sampling," Journal of American Statistical Association. Vol. 95, no. 449, pp. 135-143.

175. Heidelberger, P. 1995. "Fast Simulation of Rare Events in Queueing and Reliability Models," ACM Transactions on Modeling and Computer Simulation. Vol. 5, no. 1, pp. 43-85.

176. Shahabuddin, P. 1994. "Importance Sampling for the Simulation of Highly Reliable Markovian Systems," Management Science. Vol. 40, no. 3, pp. 333-352.

177. Goyal, A., P. Shahabuddin, P. Heidelberger, V.F. Nicola, and P.W. Glynn. 1992. "A Unified Framework for Simulating Markovian Models of Highly Dependable Systems," IEEE Transactions on Computers. Vol. 41, no. 1, pp. 36-51.

178. Melchers, R.E. 1990. "Search-Based Importance Sampling," Structural Safety. Vol. 9, no. 2, pp. 117-128.

179. Glynn, P.W. and D.L. Iglehart. 1989. "Importance Sampling for Stochastic Simulations," Management Science. Vol. 35, no. 11, pp. 1367-1392.

180. Nuclear Energy Institute v. Environmental Protection Agency. 373 F.3d 1 (D.C. Cir. 2004) (NEI) (Docket no. OAR-2005-0083-0080).

181. U.S. EPA (U.S. Environmental Protection Agency). 2005. "40 CFR Part 197: Public Health and Environmental Radiation Protection Standards for Yucca Mountain, Nevada; Proposed Rule," Federal Register. Vol. 10, no. 161, pp. 49014-49065.

182. U.S. NRC (U.S. Nuclear Regualtory Commission). 2005. "10 CFR Part 63: Implementation of a Dose Standard after 10,000 Years," Federal Register. Vol. 70, no. 173, pp. 53313-53320. 


\section{DISTRIBUTION}

\section{External Distribution}

Prof. Mark D. Abkowitz

Dept. of Civil and Environmental Engineering Vanderbilt University

Nashville, TN 37235

Prof. Joonhong Ahn

Department of Nucear Engineering

University of California, Berkeley

Berkeley, CA 94720

Prof. G. E. Apostolakis

Department of Nuclear Engineering

Massachusetts Institute of Technology

Cambridge, MA 02139-4307

Prof. Bilal Ayyub

University of Maryland

Center for Technology \& Systems Management

Civil \& Environmental Engineering

Rm. 0305 Martin Hall

College Park, MD 20742-3021

Prof. Ivo Babuska

TICAM

Mail Code C0200

University of Texas at Austin

Austin, TX 78712-1085

Timothy M. Barry

National Center for Environmental Economics

U.S. Environmental Protection Agency

1200 Pennsylvania Ave., NW

MC 1809

Washington, DC 20460

Steven M. Bartell

The Cadmus Group, Inc.

339 Whitecrest Dr.

Maryville, TN 37801

Bechtel SAIC Company, LLC (2)

Attn: Bob Andrews

Rob Howard

1180 Town Center Drive

Las Vegas, NV 89134

Prof. Bruce Beck

University of Georgia

D.W. Brooks Drive

Athens, GA 30602-2152
Prof. James Berger

Inst. of Statistics and Decision Science

Duke University

Box 90251

Durham, NC 27708-0251

Prof. Daniel Berleant

Iowa State University

Department of EE \& CE

2215 Coover Hall

Ames, IA 50014

Prof. V. M. Bier

Department of Industrial Engineering

University of Wisconsin

Madison, WI 53706

Prof. S.M. Blower

Department of Biomathematics

UCLA School of Medicine

10833 Le Conte Avenue

Los Angeles, CA 90095-1766

Prof. W. J. Conover

College of Business Administration

Texas Tech. University

Lubbock, TX 79409

Prof. Allin Cornel1

Department of Civil and Environmental

Engineering

Terman Engineering Center

Stanford University

Stanford, CA 94305-4020

Prof. Alison Cullen

University of Washington

Box 353055

208 Parrington Hall

Seattle, WA 98195-3055

Prof. U. M. Diwekar

Center for Uncertain Systems, Tools for Optimization, and Management

Vishwamitra Research Institute

Westmont, IL 60559 
Prof. David Draper

Applied Math \& Statistics

147 J. Baskin Engineering Bldg.

University of California

1156 High St.

Santa Cruz, CA 95064

Prof. Isaac Elishakoff

Dept. of Mechanical Engineering

Florida Atlantic University

777 Glades Road

Boca Raton, FL 33431-0991

Prof. Donald Estep

Departmentof Mathematics

Colorado State University

Fort Collins, CO 80523

Prof. John Evans

Harvard Center for Risk Analysis

718 Huntington Avenue

Boston, MA 02115

Prof. Rodney C. Ewing

Nuclear Engineering and Radiological Science

University of Michigan

Ann Arbor, MI 48109-2104

Prof. Charles Fairhurst

$4175^{\text {th }}$ Avenue N

South Saint Paul, MN 55075

Scott Ferson

Applied Biomathematics

100 North Country Road

Setauket, New York 11733-1345

James J. Filliben

Statistical Engineering Division

ITL, M.C. 8980

100 Bureau Drive, N.I.S.T.

Gaithersburg, MD 20899-8980

Jeffrey T. Fong

Mathematical \& Computational Sciences Division

M.C. 8910

100 Bureau Drive, N.I.S.T.

Gaithersburg, MD 20899-8910

Michael V. Frank

Safety Factor Associates, Inc.

1410 Vanessa Circle, Suite 16

Encinitas, CA 92024
Prof. C. Frey

Department of Civil Engineering

Box 7908, NCSU

Raleigh, NC 27659-7908

B. John Garrick

221 Crescent Bay Dr.

Laguna Beach, CA 92651

Prof. Roger Ghanem

254C Kaprielian Hall

Dept. of Civil Engineering

3620 S. Vermont Ave.

University of Southern California

Los Angles, CA 90089-2531

Prof. James Glimm

Dept. of Applied Math \& Statistics

P138A

State University of New York

Stony Brook, NY 11794-3600

Prof. Ramana Grandhi

Dept. of Mechanical and Materials

Engineering

3640 Colonel Glenn Hwy.

Dayton, OH 45435-0001

Michael B. Gross

415 Riviera Dr.

San Rafael, CA 94901

Prof. Raphael Haftka

Dept. of Aerospace and Mechanical

Engineering and Engineering Science

P.O. Box 116250

University of Florida

Gainsville, F L 3261 1-6250

Prof. Yacov Y. Haimes

Center for Risk Management of Engineering Systems

D111 Thornton Hall

University of Virginia

Charlottesville, VA 22901

Prof. David M. Hamby

Department of Nuclear Engineering and Radiation Health Physics

Oregon State University

Corvallis, OR 97331 
Prof. Richard Hills

New Mexico State University

College of Engineering, MSC 3449

P.O. Box 30001

Las Cruces, NM 88003

F. Owen Hoffman

SENES

102 Donner Drive

Oak Ridge, TN 37830

Prof. Steve Hora

Institute of Business and Economic Studies

University of Hawaii, Hilo

523 W. Lanikaula

Hilo, HI 96720-409 1

Prof. G. M. Hornberger

Dept. of Environmental Science

University of Virginia

Charlottesville, VA 22903

R.L. Iman

Southwest Design Consultants

12005 St. Mary’s Drive, NE

Albuquerque, NM 87111

Intera, Inc. (2)

Attn: Neal Deeds Srikanta Mishra

9111A Research Blvd.

Austin, TX 78758

Prof. Andrew Kadak

Department of Nuclear Engineering

Massachusetts Institute of Technology

Cambridge, MA 02139-4307

Prof. W. E. Kastenberg

Department of Nuclear Engineering

University of California, Berkeley

Berkeley, CA 94720

John Kessler

HLW and Spent Fuel Management Program

Electric Power Research Institute

1300 West W.T. Harris Blvd.

Charlotte, NC 28262

Prof. Peter Kitanidis

Department of Civil and Environmental Engineering

Stanford University

Stanford, CA 94305-4020
Prof. George Klir

Binghamton University

Thomas J. Watson School of Engineering \&

Applied Sciences

Engineering Building, T-8

Binghamton NY 13902-6000

Averill M. Law

6601 E. Grant Rd.

Suite 110

Tucson, AZ 85715

Prof. Sankaran Mahadevan

Vanderbilt University

Dept. of Civil and Environmental Engineering

Box 6077, Station B

Nashville, TN 37235

Don Marshall

84250 Indio Springs Drive, \#291

Indio, CA 92203

Jean Marshall

84250 Indio Springs Drive, \#291

Indio, CA 92203

Prof. Thomas E. McKone

School of Public Health

University of California

Berkeley, CA 94270-7360

Prof. Gregory McRae

Dept. of Chemical Engineering

Massachusetts Institute of Technology

Cambridge, MA 02139

Ian Miller

Goldsim Technology Group

22516 SE $64^{\text {th }}$ Place, Suite 110

Issaquah, WA 98027-5379

Prof. Max Morris

Department of Statistics

Iowa State University

304A Snedecor-Hall

Ames, IW 50011-1210

Prof. Ali Mosleh

Center for Reliability Engineering

University of Maryland

College Park, MD 207 14-21 15 
Naval Research Laboratory (4)

Attn: Jay Borris

Allen J. Goldberg

Robert Gover

John G. Michopoulos

4555 Overlook Avenue

S.W. Washington D.C. 20375

Prof. Shlomo Neuman

Department of Hydrology and Water Resources

University of Arizona

Tucson, AZ 85721

Thomas J. Nicholson

Office of Nuclear Regulatory Research

Mail Stop T-9C34

U.S. Nuclear Regulatory Commission

Washington, DC 20555

Prof. Efstratios Nikolaidis

MIME Dept.

4035 Nitschke Hall

University of Toledo

Toledo, OH 43606-3390

D. Warner North

North Works, Inc.

1002 Misty Lane

Belmont, C.A. 94002

Nuclear Waste Technical Review Board (2)

Attn: Chairman

2300 Clarendon Blvd. Ste 1300

Arlington, VA 22201-3367

Prof. David Okrent

Mechanical and Aerospace Engineering Department

University of California

48-121 Engineering IV Building

Los Angeles, CA 90095-1587

Gareth Parry

19805 Bodmer Ave

Poolesville, MD 200837

Prof. M. Elisabeth Paté-Cornel1

Department of Industrial Engineering and Management

Stanford University

Stanford, CA 94305
Prof. Per Peterson

Department of Nuclear Engineering

University of California, Berkeley

Berkeley, CA 94720

Prof. Thomas H. Pigford

Department of Nuclear Engineering

University of California

Berkeley, CA 94720

Prof. Herschel Rabitz

Princeton University

Department of Chemistry

Princeton, NJ 08544

Prof. Adrian E. Raftery

Department of Statistics

University of Washington

Seattle, WA 98195

Kadambi Rajagopal

The Boeing Company

6633 Canoga Avenue

Canoga Park, CA 91309-7922

Prof. John Renaud

Dept. of Aerospace \& Mechanical Engr.

University of Notre Dame

Notre Dame, IN 46556

Prof. James A. Reneke

Department of Mathematical Sciences

Clemson University

Clemson, SC 29634-0975

Patrick J. Roache

1215 Apache Drive

Socorro, NM 87801

Prof. Tim Ross

Dept. of Civil Engineering

University of New Mexico

Albuquerque, NM 87131

Prof. J. Sacks

Inst. of Statistics and Decision Science

Duke University

Box 90251

Durham, NC 27708-0251

Nell Sedransk

Statistical Engineering Division ITL, M.C. 8980

100 Bureau Drive, N.I.S.T.

Gaithersburg, MD 20899-8980 
Prof. Nozer D. Singpurwalla

The George Washington University

Department of Statistics

2140 Pennsylvania Ave. NW

Washington, DC 20052

Nathan Siu

Probabilistic Risk Analysis Branch

MS 10E50

U.S. Nuclear Regulatory Commission

Washington, DC 20555-0001

Southwest Research Institute (8)

Attn: C.E. Anderson

C.J. Freitas

L. Huyse

S. Mohanty

O. Osidele

O. Pensado

B. Sagar

B. Thacker

P.O. Drawer 28510

622 Culebra Road

San Antonio, TX 78284

Prof. C.B. Storlie

Department of Mathematics and Statistics

University of New Mexico

Albuquerque, NM 87131-0001

Prof. T. G. Theofanous

Department of Chemical and Nuclear Engineering

University of California

Santa Barbara, CA 93106

Prof. K.M. Thompson

Harvard School of Public Health

677 Huntington Avenue

Boston, MA 02115

Martin Tierney

Plantae Research Associates

415 Camino Manzano

Santa Fe, NM 87505

Prof. Fulvio Tonon

Department of Civil Engineering

University of Texas at Austin

1 University Station C1792

Austin, TX 78712-0280
Stephen D. Unwin

Pacific Northwest National Laboratory

P.O. Box 999

Mail Stop K6-52

Richland, WA 99354

U.S. Nuclear Regulatory Commission (6)

Office of Nuclear Material Safety and Safeguards

Attn: A.C. Campbell (MS TWFN-7F27)

R.B. Codell (MS TWFN-7F27)

K.W. Compton (MS TWFN-7F27)

S.T. Ghosh (MS TWFN-7F27)

B.W. Leslie (MS TWFN-7F27)

T.J. McCartin (MS TWFN-7F3)

Washington, DC 20555-0001

Christopher G. Whipple

Environ

Marketplace Tower

6001 Shellmound St. Suite 700

Emeryville, C.A. 94608

Prof. Dongxiao Zhang

Mewbourne School of Petroleum and

Geological Engineering

The University of Oklahoma

Norman, OK 73019

Foreign Distribution

Jesus Alonso

ENRESA

Calle Emillo Vargas 7

28043 MADRID

SPAIN

Prof. Tim Bedford

Department of Management Sciences

Strathclyde University

40 George Street

Glasgow G630NF

UNITED KINGDOM

Prof. Yakov Ben-Haim

Department of Mechanical Engineering

Technion-Israel Institute of Technology

Haifa 32000

ISRAEL

Prof. Ricardo Bolado

Polytechnical University of Madrid

Jose Gutierrez, Abascal, 2

28006 Madrid

SPAIN 
Prof. A.P. Bourgeat

UMR 5208 - UCB Lyon1, MCS, Bât. ISTIL

Domaine de la Doua; 15 Bd. Latarjet

69622 Villeurbanne Cedex

FRANCE

Prof. D.G. Cacuci

Institute for Nuclear Technology and Reactor Safety

University of Karlsruhe

76131 Karlsruhe

GERMANY

Prof. Enrique Castillo

Department of Applied Mathematics and Computational Science

University of Cantabria

Santandar

SPAIN

CEA Cadarache (2)

Attn: Nicolas Devictor

Bertrand Iooss

DEN/CAD/DER/SESI/CFR

Bat 212

13108 Saint Paul lez Durance cedex

FRANCE

Prof. Russell Cheng

School of Mathematics

Southampton University

Southampton, SO17 1BJ

UNITED KINGDOM

Prof. Roger Cooke

Department of Mathematics

Delft University of Technology

P.O. Box 50312800 GA Delft

THE NETHERLANDS

Etienne de Rocquigny

EDF R\&D MRI/T56

6 quai Watier

78401 Chatou Cedex

FRANCE

Prof. Christian Ekberg

Chalmers University of Technology

Department of Nuclear Chemistry

41296 Goteborg

SWEDEN
European Commission (5)

Attn: Francesca Campolongo

Mauro Ciechetti

Marco Ratto

Andrea Saltelli

Stefano Tarantola

JRC Ispra, ISIS

21020 Ispra

ITALY

Régis Farret

Direction des Risques Accidentels

INERIS

BP2 - 60550 Verneuil en Halatte

FRANCE

Forshunginstitute GRS (2)

Attn: Eduard Hofer

B. Kryzkacz-Hausmann

Forschungsgelande Nebau 2

85748 Garching

GERMANY

Forschungszentrum Karlsruhe (2)

Attn: F. Fischer

J. Ehrhardt

Inst. Kern \& Energietechn

Postfach 3640, D-76021

Karlsruhe

GERMANY

Prof. Simon French

School of Informatics

University of Manchester

Coupland 1

Manchester M13 9pl

UNITED KINGDOM

Daniel A. Galson (2)

Galson Science Ltd.

5 Grosvenor House

Melton Rd.

Rutland LE15 6AX

ENGLAND

Prof. Louis Goossens

Safety Science Group

Delft University of Technology

P.O. Box 50312800 GA Delft

THE NETHERLANDS 
Prof. Jim Hall

University of Bristol

Department of Civil Engineering

Queens Building, University Walk

Bristol UK 8581TR

Mikhail Iosjpe

Protection Authority

Norwegian Radiation

Grini Naringspark 13

P.O. Box 55

1332 Oesteraas

NORWAY

J. Jaffré

INRIA - Roquencourt

B.P. 105

78153 Le Chesnay Cedex

FRANCE

Arthur Jones

Nat. Radio. Prot. Board

Chilton, Didcot

Oxon OX110RQ

UNITED KINGDOM

Prof. J.P.C. Kleijnen

Department of Information Systems

Tilburg University

5000 LE Tilburg

THE NETHERLANDS

Prof. S.E. Magnusson

Lund University

P.O. Box 118

22100 Lund

SWEDEN

Prof. Alex Thierry Mara

Université de la Réunion

Lab. De Génie Industriel

15, Avenue René Cassin

BP 7151

97715 St. Denis

La Réunion

FRANCE

Jan Marivoet

Centre d'Etudes de L'Energie

Nucleaire

Boeretang 200

2400 MOL

BELGIUM
Prof. Ghislain de Marsily

University Pierre et Marie Curie

Laboratorie de Geologie Applique

4, Place Jussieu

T.26 - 5e etage

75252 Paris Cedex 05

FRANCE

Prof. A. O’Hagan

Department of Probability and Statistics

University of Sheffield

Hicks Building

Sheffield S3 7RH

UNITED KINGDOM

Prof. Roberto Pastres

University of Venice

Dorsuduro 2137

30123 Venice

Dorsuduro 2137

ITALY

Prof. Leslie R. Pendrill

SP Swedish National Testing \& Research Institute

Measurement Technology, Head of Research

Box 857, S-501 15 BORÅS

SWEDEN

Guillaume Pepin

ANDRA - Service DS/CS

Parc de la Croix Blanche

$1 / 7$ rue Jean Monnet

92298 Chatenay-Malabry Cedex

FRANCE

Prof. G.I. Schuëller

Institute of Engineering Mechanics

Leopold-Franzens University

Technikerstrasse 13

6020 Innsbruck

AUSTRIA

Prof. Marian Scott

Department of Statistics

University of Glasgow

Glasgow G12 BQW

UNITED KINGDOM

Prof. Ilya Sobol'

Russian Academy of Sciences

Miusskaya Square

125047 Moscow

RUSSIA 
Prof. Wolfgang Stummer

Dept. of Mathematics

Friedrich-Alexander University

Bismarkstr. 1 1/2

91054 Erlangen

GERMANY

Prof. Tamas Turanyi

Eotvos University (ELTE)

P.O. Box 32

1518 Budapest

HUNGARY

Prof. Willem Van Groenendaal

Tilburg University

P.O. Box 90153

5000 LE Tilburg

THE NETHERLANDS

Prof. H.P. Wynn

Department of Statistics

London School of Economics

Houghton Street

London WC2A 2AE

UNITED KINGDOM

Prof. Enrico Zio

Politecnico di Milano

Via Ponzia 3413

20133 Milan

ITALY

\section{Department of Energy Laboratories}

Battelle Memorial Institute, Pacific Northwest Labs (1)

P.O. Box 999

Richland, WA 99352

Attn: Pamela Doctor

Lawrence Livermore National Laboratory (2)

Earth Sciences Division, MS 90-1116

1 Cyclotron Rd.

Berkeley,CA 94720

Attn: Y. Tsang

Lawrence Livermore National Laboratory (5)

7000 East Ave.

P.O. Box 808

Livermore, CA 94550

Attn: Jim Blink, MS LL-423

Kenneth T. Bogen, MS L-396

Robert J. Budnitz, MS L-632

Frank Graziani, MS L-095

Charles Tong, MS L-560
Los Alamos National Laboratory (12)

Mail Station 5000

P.O. Box 1663

Los Alamos, NM 87545

Attn: Mark C. Anderson, MS T080

Scott Doebling, MS T080

Francois Hemez, MS T006

David Higdon, MS F600

James Hyman, MS B284

Cliff Joslyn, MS B265

Jonathan Lucero, MS C926

Bruce Robinson, MS T003

Mandy Rutherford, MS T080

David Sigeti, MS F645

Kari Sentz, MS F600

Alyson G. Wilson, MS F600

Pacific Northwest National Laboratory (3)

Risk and Decision Science Group

Richland, WA 99352-2458

Attn: David W. Engel

Paul W. Eslinger

Brady Hanson

U.S. Department of Energy (1)

Attn: Jamileh Soudah, NA-114

Forrestal Building

1000 Independence Ave., SW

Washington, DC 20585

U.S. Department of Energy (6)

Yucca Mountain Site Characterization Office

Attn: William Boyle

Russ Dyer

Claudia Newbury

Mark Tynan

Abraham VanLuik

Michael Voegele

1551 Hillshire Drive

Las Vegas, NV 89134

\section{Sandia Internal Distribution}

$\begin{array}{llll}1 & \text { MS 1231 } & 0310 & \text { A. L. Camp } \\ 1 & \text { MS 1056 } & 1112 & \text { S. M. Meyers } \\ 1 & \text { MS 1415 } & 1120 & \text { C. J. Barbour } \\ 1 & \text { MS } 1146 & 1384 & \text { P. J. Griffin } \\ 1 & \text { MS 0310 } & 1400 & \text { G. S. Davidson } \\ 1 & \text { MS 0370 } & 1411 & \text { S. A. Mitchell } \\ 1 & \text { MS 0370 } & 1411 & \text { M. S. Eldred } \\ 1 & \text { MS } 1111 & 1411 & \text { P. Knupp } \\ 1 & \text { MS 0370 } & 1411 & \text { L. P. Swiler } \\ 1 & \text { MS 0370 } & 1411 & \text { T. G. Trucano } \\ 1 & \text { MS 0824 } & 1500 & \text { T.Y. Chu } \\ 1 & \text { MS 0828 } & 1544 & \text { M. Pilch }\end{array}$




\begin{tabular}{|c|c|c|c|c|c|c|c|}
\hline 1 & MS 0828 & 1544 & K. J. Dowding & 1 & MS 0778 & 6781 & S. Miller \\
\hline 1 & MS 0828 & 1544 & A. A. Giunta & 1 & MS 0776 & 6782 & K. Economy \\
\hline 50 & MS 0779 & 1544 & J. C. Helton & 1 & MS 0776 & 6782 & G. Freeze \\
\hline 1 & MS 0828 & 1544 & W. L. Oberkampf & 1 & MS 0776 & 6782 & T. Hadgu \\
\hline 1 & MS 0828 & 1544 & J. R. Red-Horse & 1 & MS 0776 & 6782 & H. Iuzzolino \\
\hline 1 & MS 0828 & 1544 & V. J. Romero & 1 & MS 0748 & 6782 & E. A. Kalinina \\
\hline 1 & MS 1153 & 5441 & L. C. Sanchez & 1 & MS 0776 & 6782 & S. Kuzio \\
\hline 1 & MS 0724 & 6000 & L. E. Shephard & 1 & MS 1399 & 6782 & M. G. Marietta \\
\hline 1 & MS 1138 & 6222 & P. G. Kaplan & 1 & MS 0776 & 6782 & R. McCurley \\
\hline 1 & MS 0615 & 6252 & J. A. Cooper & 1 & MS 0776 & 6782 & J. Schreiber \\
\hline 1 & MS 0706 & 6311 & M. G. Wallace & 1 & MS 0776 & 6782 & J. S. Stein \\
\hline 1 & MS 0757 & 6442 & J. L. Darby & 1 & MS 0776 & 6782 & B. Walsh \\
\hline 1 & MS 0757 & 6442 & G. D. Wyss & 1 & MS 0776 & 6782 & Y. Wang \\
\hline 1 & MS 1011 & 6642 & D. J. Anderson & 1 & MS 0776 & 6783 & E. J. Bonano \\
\hline 1 & MS 1011 & 6642 & M. S. Shortencarrier & 1 & MS 0779 & 6783 & L. Dotson \\
\hline 1 & MS 1011 & 6643 & R. M. Cranwell & 1 & MS 0776 & 6783 & R. J. MacKinnon \\
\hline 1 & MS 0701 & 6700 & P. B. Davies & 1 & MS 1399 & 6783 & M. A. Martell \\
\hline 1 & MS 1395 & 6710 & D. Kessel & 1 & MS 1399 & 6783 & S. Mehta \\
\hline 1 & MS 1395 & 6711 & J. W. Garner & 1 & MS 0776 & 6783 & R. P. Rechard \\
\hline 1 & MS 0776 & 6711 & A. Gilkey & 1 & MS 1399 & 6783 & D. Sevougian \\
\hline 1 & MS 1395 & 6711 & J. Kanney & 1 & MS 0778 & 6783 & P. N. Swift \\
\hline 1 & MS 1395 & 6711 & T. Kirchner & 1 & MS 0776 & 6783 & M. Tierney \\
\hline 1 & MS 1395 & 6711 & C. Leigh & 1 & MS 1399 & 6783 & P. Vaughn \\
\hline 1 & MS 1395 & 6711 & M. Nemer & 1 & MS 1399 & 6783 & J. Younkers \\
\hline 1 & MS 0776 & 6711 & D. Rudeen & 1 & MS 1399 & 6784 & B. Bullard \\
\hline 1 & MS 1395 & 6711 & E. Vugrin & 1 & MS L423 & 6784 & J. McNeish \\
\hline 1 & MS 1395 & 6711 & K. Vugrin & 1 & MS 0776 & 6784 & P. Mattie \\
\hline 1 & MS 1395 & 6712 & M. Rigali & 1 & MS 1399 & 6784 & C. B. Thom \\
\hline 1 & MS 1395 & 6712 & R. Beauheim & 1 & MS 0776 & 6784 & C. Sallaberry \\
\hline 1 & MS 1395 & 6712 & L. Brush & 1 & MS 1399 & 6784 & E. Zawahler \\
\hline 1 & MS 0778 & 6712 & P. Domski & 1 & MS 0771 & 6785 & F. Hansen \\
\hline 1 & MS 0779 & 6725 & H.-N. Jow & 1 & MS 1399 & 6785 & E. Hardin \\
\hline 1 & MS 0776 & 6728 & A. Reed & 1 & MS 1399 & 6785 & C. Howard \\
\hline 1 & MS 0748 & 6761 & D. G. Robinson & 1 & MS 0778 & 6785 & C. Bryan \\
\hline 1 & MS 0748 & 6761 & S. P. Burns & 1 & MS 0778 & 6785 & R. L. Jarek \\
\hline 1 & MS 0748 & 6762 & N. Bixler & 1 & MS 0778 & 6785 & P. Mariner \\
\hline 1 & MS 0748 & 6762 & R. Gauntt & 1 & MS 1399 & 6785 & K. Mon \\
\hline 1 & MS 0771 & 6770 & J. E. Kelly & 1 & MS 0830 & 12335 & K. V. Diegert \\
\hline 1 & MS 0736 & 6770 & D. A. Powers & 1 & MS 0829 & 12337 & B. M. Rutherford \\
\hline 1 & MS 0779 & 6772 & C. Axness & 1 & MS 0829 & 12337 & F. W. Spencer \\
\hline 1 & MS 0779 & 6772 & J. Johnson & 1 & MS 0405 & 12346 & S. E. Camp \\
\hline 1 & MS 0779 & 6772 & J. Jones & 1 & MS 0405 & 12347 & L-J Shyr \\
\hline 1 & MS 1399 & 6780 & A. Orrell & 1 & MS 0405 & 12347 & T. D. Brown \\
\hline 1 & MS 0778 & 6781 & B. W. Arnold & 1 & MS 0405 & 12347 & R. D. Waters \\
\hline 1 & MS 0778 & 6781 & C. Jove-Colon & 2 & MS 9018 & 8944 & Central Technical \\
\hline 1 & MS 1399 & 6781 & C. W. Hansen & & & & Files \\
\hline 1 & MS 0776 & 6781 & M. K. Knowles & 2 & MS 0899 & 4536 & Technical Library \\
\hline
\end{tabular}

